\title{
coatings
}

Advanced

Strategies in Thin

Film Engineering

by Magnetron

Sputtering

Edited by

Alberto Palmero and Nicolas Martin

Printed Edition of the Special Issue Published in Coatings 


\section{Advanced Strategies in Thin Film Engineering by Magnetron Sputtering}





\section{Advanced Strategies in Thin Film Engineering by Magnetron Sputtering}

Special Issue Editors

Alberto Palmero

Nicolas Martin 
Special Issue Editors

Alberto Palmero

Spanish Council of Research (CSIC)

Spain

Nicolas Martin

FEMTO-ST Institute, University of Bourgogne Franche-Comté

France

\section{Editorial Office}

MDPI

St. Alban-Anlage 66

4052 Basel, Switzerland

This is a reprint of articles from the Special Issue published online in the open access journal Coatings (ISSN 2079-6412) (available at: https://www.mdpi.com/journal/coatings/special_issues/ film_Magnetron_sputter).

For citation purposes, cite each article independently as indicated on the article page online and as indicated below:

LastName, A.A.; LastName, B.B.; LastName, C.C. Article Title. Journal Name Year, Article Number, Page Range.

ISBN 978-3-03936-429-9 (Hbk)

ISBN 978-3-03936-430-5 (PDF)

Cover image courtesy of Raya El Beainou, Ph.D. Thesis 2019, Univ. Bourgogne Franche-Comté, France.

(C) 2020 by the authors. Articles in this book are Open Access and distributed under the Creative Commons Attribution (CC BY) license, which allows users to download, copy and build upon published articles, as long as the author and publisher are properly credited, which ensures maximum dissemination and a wider impact of our publications.

The book as a whole is distributed by MDPI under the terms and conditions of the Creative Commons license CC BY-NC-ND. 


\section{Contents}

About the Special Issue Editors $\ldots \ldots \ldots \ldots \ldots \ldots \ldots$ vii

Alberto Palmero and Nicolas Martin

Advanced Strategies in Thin Films Engineering by Magnetron Sputtering

Reprinted from: Coatings 2020, 10, 419, doi:10.3390/coatings10040419 .

Amine Achour, Mohammad Islam, Iftikhar Ahmad, Khalid Saeed and Shahram Solaymani Electrochemical Stability Enhancement in Reactive Magnetron Sputtered VN Films upon Annealing Treatment

Reprinted from: Coatings 2019, 9, 72, doi:10.3390/coatings9020072 _ . . . . . . . . . . . 7

Jan-Ole Achenbach, Stanislav Mráz, Daniel Primetzhofer and Jochen M. Schneider

Correlative Experimental and Theoretical Investigation of the Angle-Resolved Composition

Evolution of Thin Films Sputtered from a Compound $\mathrm{Mo}_{2} \mathrm{BC}$ Target

Reprinted from: Coatings 2019, 9, 206, doi:10.3390/coatings9030206

Florian G. Cougnon and Diederik Depla

The Seebeck Coefficient of Sputter Deposited Metallic Thin Films: The Role of Process Conditions

Reprinted from: Coatings 2019, 9, 299, doi:10.3390/coatings9050299

Manuela Proença, Marco S. Rodrigues, Joel Borges and Filipe Vaz

Gas Sensing with Nanoplasmonic Thin Films Composed of Nanoparticles (Au, Ag) Dispersed in a CuO Matrix

Reprinted from: Coatings 2019, 9, 337, doi:10.3390/coatings9050337 . . . . . . . . . . . . .

Sunil Babu Eadi, Hyeong-Sub Song, Hyun-Dong Song, Jungwoo Oh and Hi-Deok Lee

Nickel Film Deposition with Varying RF Power for the Reduction of Contact Resistance in NiSi

Reprinted from: Coatings 2019, 9, 349, doi:10.3390/coatings9060349 . . . . . . . . . . . . 55

Hui Liang, Xi Geng, Wenjiang Li, Adriano Panepinto, Damien Thiry, Minfang Chen and Rony Snyders

Experimental and Modeling Study of the Fabrication of Mg Nano-Sculpted Films by Magnetron Sputtering Combined with Glancing Angle Deposition

Reprinted from: Coatings 2019, 9, 361, doi:10.3390/coatings9060361 . . . . . . . . . . . . . .

Raúl Gago, Slawomir Prucnal, René Hübner, Frans Munnik, David Esteban-Mendoza, Ignacio Jiménez and Javier Palomares

Phase Selectivity in $\mathrm{Cr}$ and $\mathrm{N}$ Co-Doped $\mathrm{TiO}_{2}$ Films by Modulated Sputter Growth and Post-Deposition Flash-Lamp-Annealing

Reprinted from: Coatings 2019, 9, 448, doi:10.3390/coatings9070448 . . . . . . . . . . . 77

Cao Phuong Thao, Dong-Hau Kuo, Thi Tran Anh Tuan, Kim Anh Tuan, Nguyen Hoang Vu, Thach Thi Via Sa Na, Khau Van Nhut and Nguyen Van Sau

The Effect of RF Sputtering Conditions on the Physical Characteristics of Deposited GeGaN Thin Film

Reprinted from: Coatings 2019, 9, 645, doi:10.3390/coatings9100645 . . . . . . . . . . . . 91

Tran Anh Tuan Thi, Dong-Hau Kuo, Phuong Thao Cao, Pham Quoc-Phong,

Vinh Khanh Nghi and Nguyen Phuong Lan Tran

Electrical and Structural Properties of All-Sputtered Al/ $\mathrm{SiO}_{2} / p-\mathrm{GaN}$ MOS Schottky Diode

Reprinted from: Coatings 2019, 9, 685, doi:10.3390/coatings9100685 . . . . . . . . . . . . 101 
Thi Tran Anh Tuan, Dong-Hau Kuo, Phuong Thao Cao, Van Sau Nguyen, Quoc-Phong Pham, Vinh Khanh Nghi and Nguyen Phuong Lan Tran

Electrical Characterization of RF Reactive Sputtered $p-\mathrm{Mg}-\mathrm{In}_{x} \mathrm{Ga}_{1-x} \mathrm{~N} / n-\mathrm{Si}$ Hetero-Junction Diodes without Using Buffer Layer

Reprinted from: Coatings 2019, 9, 699, doi:10.3390/coatings9110699 . . . . . . . . . . . . . . 111

Ihar Saladukhin, Gregory Abadias, Vladimir Uglov, Sergey Zlotski, Arno Janse van Vuuren and Jacques Herman O'Connell

Structural Properties and Oxidation Resistance of $\mathrm{ZrN} / \mathrm{SiN}_{\mathrm{x}}, \mathrm{CrN} / \mathrm{SiN}_{\mathrm{x}}$ and $\mathrm{AlN} / \mathrm{SiN}_{\mathrm{x}}$ Multilayered Films Deposited by Magnetron Sputtering Technique

Reprinted from: Coatings 2020, 10, 149, doi:10.3390/coatings10020149 . . . . . . . . . . . . . . . 121 


\title{
About the Special Issue Editors
}

\begin{abstract}
Alberto Palmero is a Tenured Scientist at the Institute of Materials Science of Seville, a joint center between the Spanish Council of Research (CSIC) and the University of Seville. He started his research career at the University of Seville (Spain), where he carried out his Ph.D. on the modelling and characterization of argon and oxygen plasmas employed to grow thin films by chemical and physical vapor deposition techniques (2002). After that, he continued his activity at Utrecht University (The Netherlands, 2002-2006), where he studied the plasma-assisted reactive magnetron sputtering technique and, in particular, the transport of ballistic and diffusive species in plasma gas and the associated thermal phenomena. In 2008, he got a permanent position at the Institute of Materials Science of Seville (Spain), where he led a research group aimed at fine tuning atomistic phenomena on surfaces to grow "a la carte" thin films. In 2014, he was elected Head of the "Nanostructured Functional Materials" department at the Board of the Institute of Materials Science of Seville (2014-2018). His research encompasses several areas, from plasma dynamics and plasma-surface interaction, to the description of surface nanostructuration phenomena in dynamic, far from equilibrium, situations. A key aspect of his activity is the combined approach of computer simulations and fundamental experiments to achieve accurate control of the film nanostructure, as well as the subsequent development of prototype functional devices. He has authored more than 70 publications, written 10 book chapters and has been invited to give keynote presentations at numerous international conferences and symposia. He has also authored four patents, and has won several prizes on research and innovation.
\end{abstract}

Nicolas Martin obtained a Ph.D. in Physical Chemistry from the University of Franche-Comté in 1997 and a habilitation degree (Docent) from the same university in 2005. He was a researcher at the Ecole Polytechnique Fédérale de Lausanne (Switzerland) from 1998 to 2000 in the Physics department. He got a permanent position as Associate Professor at the National Engineering School of the Ecole Nationale Supérieure de Mécanique et des Microtechniques (ENSMM) in Besançon in 2000. He became Full Professor of Materials Science in 2008. He was a visiting researcher from 2012-2013 at the University of Uppsala (Sweden), where he worked at the Angström Laboratory in the Department of Engineering Sciences, Solid State Electronics. In 2017, he spent a short sabbatical leave in the University of Alberta in Edmonton (Canada), to work in the Department of Electrical and Computer Engineering. His research is focused on the physics and technology of metallic and ceramic thin films prepared by reactive sputtering. He is also interested in nanostructuration of coatings prepared by Glancing Angle Deposition (GLAD). He was the head of the MIcro NAno MAterials \& Surfaces (MINAMAS) team in the Micro Nano Sciences \& Systems (MN2S) research department of the FEMTO-ST Institute in 2008 and 2009. He was previously the Deputy Director of MN2S research department from 2010 to 2014. Nicolas Martin has authored or co-authored more than 130 articles in international peer-reviewed journals, one patent, five chapters in books, one e-book, and more than 200 presentations as part of conferences, workshops and short courses. 

Editorial

\title{
Advanced Strategies in Thin Films Engineering by Magnetron Sputtering
}

\author{
Alberto Palmero ${ }^{1, *}$ and Nicolas Martin ${ }^{2, *}$ \\ 1 Instituto de Ciencia de Materiales de Sevilla (CSIC-Universidad de Sevilla), Americo Vespucio 49, \\ 41092 Sevilla, Spain \\ 2 Institut FEMTO-ST, UMR 6174, CNRS, University Bourgogne Franche-comté, 15B, Avenue des Montboucons, \\ 25030 Besançon, France \\ * Correspondence: alberto.palmero@csic.es (A.P.); nicolas.martin@femto-st.fr (N.M.)
}

Received: 27 March 2020; Accepted: 20 April 2020; Published: 23 April 2020

\begin{abstract}
This Special Issue contains a series of reviews and papers representing some recent results and some exciting perspectives focused on advanced strategies in thin films growth, thin films engineering by magnetron sputtering and related techniques. Innovative fundamental and applied research studies are then reported, emphasizing correlations between structuration process parameters, new ideas and approaches for thin films engineering and resulting properties of as-deposited coatings.
\end{abstract}

Keywords: magnetron sputtering; nanostructures; growth mechanism; functional properties; HiPIMS; oblique angle deposition

\section{Introduction}

Thin films are the workhorses of many of today's innovative technologies. Entire processes, from organic electronics to aerospace to packing industries, are strongly dependent on thin films. There are many cases where a given property of thin films gave rise to an entirely new field of technology. During these last decades, thin films engineering has been changed from a laboratory curiosity to become a multi-billion euros industry worldwide. New production technologies and advanced techniques are introduced every year to add new tools to the thin film toolbox [1-4]. One of the most exciting motivations is to generate innovative thin films and original nanostructured thin films. For this purpose, recent years have witnessed the flourishing of numerous novel strategies based on the magnetron sputtering technique, aimed at the advanced engineering of thin films, such as HiPIMS, combined vacuum processes, the implementation of complex precursor gases, or the inclusion of particle guns in the reactor, among others [5-8]. At the forefront of these approaches, investigations focused on nanostructured coatings appear today as one of the priorities in many scientific and technological communities: The science behind them appears in most of the cases as a "terra incognita", fascinating both the fundamentalist, who imagines new concepts, and the experimenter, who is able to create and study new films with, as of yet, unprecedented performances $[9,10]$.

\section{Thin Films Engineering: Where Do We Stand?}

Scientific and technological challenges focused on thin films engineering, along with the existence of numerous scientific issues that have yet to be clarified in classical magnetron sputtering depositions (e.g., process control and stability, nanostructuration mechanisms, connection between film morphology and properties, or upscaling procedures from the laboratory to industrial scales) have motivated us to edit a specialized volume containing the state-of-the art that put together these innovative fundamental and applied research topics.

It is systematically observed that most of the scientific and technological developments are closely linked and often limited by the performance of materials and surfaces. As a result, this last decade 
has seen the development of original scientific fields related to the creation of intelligent materials, functional materials, biomaterials, etc. [11,12] Structured thin films, in particular, are thus moved from laboratory curiosity to objects of high added value. They are becoming a science in themselves and complete technologies may now depend on their properties and their integration [13-15]. Various fields, such as electronics, space vehicles, decorative, etc., are highly dependent on materials and their functionality. In many cases, the scientific observation of a characteristic of a material led to the creation of a new technology. New production systems and techniques are advanced and implemented each year to create new performances in the current rush to multifunctional surfaces and materials. As a result, it became a scientific requirement to provide new opportunities for the development of components and innovative structured materials.

At the forefront of many scientific strategies, investigations focused on the surfaces and structured materials appear today as one of the priorities of many laboratories. If some groups are devoting considerable efforts to the study of nano-scaled objects, or inversely, to systems of a few tens of micrometers, the components of intermediate sizes located between the nano- and micrometer remain a "gap" of knowledge to explore. This window size appears as a "terra incognita", fascinating both for the fundamentalist, who imagines new concepts, but also for the experimenter, who is able to create and study components with unprecedented performances. It is in this dimensional window spanning the nano- to micrometer that thin films engineering strategies become more than relevant and definitely provide an extra dimension in the current race to expand the range of thin film properties.

\section{This Special Issue}

This Special Issue, entitled "Advanced strategies in thin films engineering by magnetron sputtering", contains five reviews and six research articles covering fundamental investigations, as well as applied research studies devoted to nanostructuration and thin films engineering produced by magnetron sputtering and related deposition methods. Without going into detail, the individual work is briefed below:

The structure, stress state and phase composition of $\mathrm{MeN}_{\mathrm{SiN}}(\mathrm{Me}=\mathrm{Zr}, \mathrm{Cr}, \mathrm{Al})$ multilayered films are reviewed by Saladuhkin et al. [16] The stability of the coatings to oxidation is studied as a function of the thickness of sub-layers at the nanometric scale. The oxidation resistance of $\mathrm{MeN} / \mathrm{SiN}_{x}$ multilayers is significantly improved compared to reference monolithic films, especially by increasing the fraction of $\mathrm{SiN}_{\mathrm{x}}$ layer thickness. An optimized performance is obtained for $\mathrm{CrN} / \mathrm{SiN}$ and $\mathrm{AlN} / \mathrm{SiN} \mathrm{N}_{x}$ with nanometric periods, which remain stable up to $950^{\circ} \mathrm{C}$.

Liang et al. [17] report on the preparation of $\mathrm{Mg}$ nano-sculpted thin films by magnetron sputtering, implementing the glancing angle deposition technique. They demonstrate how the microstructure of the film can be tuned by adjusting deposition parameters such as the tilt angle or the sputtering pressure, which both largely influence the shadowing effect during the film deposition. They also model the growth of the material using kinetic Monte Carlo approaches, which prove the role of surface diffusion during the preparation of the film.

The paper "Gas Sensing with Nanoplasmonic Thin Films Composed of Nanoparticles (Au, Ag) dispersed in a $\mathrm{CuO}$ matrix" by Proença et al. presents original and interesting nano-plasmonic platforms capable of detecting the presence of gas molecules [18]. The authors show that the localized surface plasmon resonance phenomenon, LSPR, is produced by the morphological changes of the nanoparticles (size, shape, and distribution modified by thermal annealing of the films). Such an approach can be used to improve the sensitivity to the gas molecules, with the highest sensing performances for the bimetallic films.

Cougnon and Depla [19] develop thin film thermocouples as a potential way to embed sensors in composite systems, especially for their application in lightweight and smart structures. They experimentally investigate the influence of the discharge current and residual gas impurities on the Seebeck coefficient for sputtered copper and constantan thin films. These deposition parameters both lead to changes in the ratio between the impurity flux to metal flux towards the growing film. Such a 
parameter is assumed to be a quantitative criterion for the background residual gas incorporation in the film, and acts as a grain refiner.

The angle-resolved composition evolution of Mo-B-C thin films deposited from a $\mathrm{Mo}_{2} \mathrm{BC}$ compound target is experimentally and theoretically investigated by Achenbach et al. [20]. The authors use TRIDYN and SIMTRA to calculate the influence of the sputtering gas on the angular distribution function of the sputtered species from the target surface, transport through the gas phase, and film composition. They show that the mass ratio between sputtering gas and sputtered species defines the scattering angle within the collision cascades in the target, as well as for the collisions in the gas phase, which influences the angle- and pressure-dependent film compositions.

The electrical and structural properties of sputter-deposited $\mathrm{p}-\mathrm{Mg}-\mathrm{In}_{x} \mathrm{Ga}_{1-x} \mathrm{~N} / \mathrm{n}$-Si hetero-junction diodes and $\mathrm{Al} / \mathrm{SiO}_{2} / \mathrm{p}-\mathrm{GaN}$ MOS Schottky diodes are studied by Tuan et al. [21,22] Electronic transport properties by means of Hall effect measurements are comprehensively performed. Holes concentration and mobility at room temperature are determined, as well as $I-V$ and $C-V$ measurements at different frequencies. Other characteristics for MOS diodes are performed and compared by Cheung's and Norde's methods.

Thao et al. [23] investigate $\mathrm{Ge}_{0.07} \mathrm{GaN}$ films prepared by radio frequency reactive sputtering changing RF sputtering power and heating temperature conditions. Structure, optical and electrical characteristics of the films are significantly affected by both deposition parameters and with the best electronic transport properties and the lowest photoenergy produced for the deposited-150 W $\mathrm{Ge}_{0.07} \mathrm{GaN}$ film.

The paper "Phase Selectivity in $\mathrm{Cr}$ and N Co-Doped $\mathrm{TiO}_{2}$ Films by Modulated Sputter Growth and Post-Deposition Flash-Lamp-Annealing" by Gago et al. presents how the interface engineering strategy can vary the phase occurrence in $\mathrm{Cr}$ and $\mathrm{N}$ co-doped $\mathrm{TiO}_{2}\left(\mathrm{TiO}_{2}: \mathrm{Cr}, \mathrm{N}\right)$ sputter-deposited films [24]. A post-deposition flash-lamp-annealing (FLA) is also used to favor anatase phase, and to give rise to dopant activation and diffusion. The authors show that using interface engineering and millisecond-range-FLA allows tailoring the structure of $\mathrm{TiO}_{2}$-based functional materials.

In order to investigate the lowering of the contact resistance in the NiSi/Si junction, Eadi et al. systematically change the RF power implemented for the sputter-deposition of Ni thin films [25]. A post-deposition rapid thermal annealing is applied for the nickel silicide fabrication and a circular transmission line model (CTLM) procedure is developed to obtain the contact resistance. They demonstrate that $\mathrm{Ni}$ film resistivity can be reduced for an optimized RF sputtering power and the formed NiSi phase shows a low contact resistance.

Achour et al. [26] report on VN thin films produced by DC reactive magnetron sputtering, followed by vacuum annealing. They apply different temperatures and study the effect on the electrochemical stability and surface chemistry of the films. They particularly focus on the oxide layer formed on the $\mathrm{VN}$ and prove that annealing of VN films makes them an attractive candidate for long-term use in electrochemical capacitors.

In summary, this Special Issue of Coatings gathers reviews and original articles illustrating the strong potential of thin films engineering for the creation of attractive and original functional coatings based on magnetron sputtering processes. This series of publications also demonstrate the fundamental role of thin films structuration at the micro- and nanoscale for understanding growth mechanisms and generating innovative behaviors of materials and surfaces.

Funding: This research received no external funding.

Acknowledgments: We would like to warmly thank all the authors, reviewers and editors for their valuable contribution in this Special Issue of Coatings.

Conflicts of Interest: The authors declare no conflict of interest. 


\section{References}

1. Gleiter, H. Nanostructured materials: Basic concepts and microstructure. Acta Mater. 2000, 48, 1-29. [CrossRef]

2. Lakhtakia, Y.; Messier, R. Sculptured Thin Films; SPIE Press: Bellingham, WA, USA, 2005. [CrossRef]

3. Fendler, J.H. Self-assembled nanostructured materials. Chem. Mater. 1996, 8, 1616-1624. [CrossRef]

4. Xi, J.Q.; Schubert, M.H.; Kim, J.K.; Schubert, E.F.; Chen, M.; Lin, S.Y.; Liu, W.; Smart, J.A. Optical thin-film materials with low refractive index for broadband elimination of Fresnel reflection. Nat. Photonics 2007, 1, 176-179. [CrossRef]

5. Schlom, D.G.; Chen, L.Q.; Pan, X.; Schmehl, A.; Zurbuchen, M.A. A thin film approach to engineering functionality into oxides. J. Am. Ceram. Soc. 2008, 91, 2429-2454. [CrossRef]

6. Bunker, B.B.; Rieke, P.C.; Tarasevich, B.J.; Campbell, A.A.; Fryxell, G.E.; Graff, G.L.; Song, L.; Liu, J.; Virden, J.W.; McVay, G.L. Ceramic thin-film formation on functionalized interfaces through biomimetic processing. Science 1994, 264, 48-55. [CrossRef]

7. Choy, K.L. Chemical vapour deposition of coatings. Prog. Mater. Sci. 2003, 48, 57-170. [CrossRef]

8. Hawkeye, M.M.; Brett, M.J. Glancing angle deposition: Fabrication, properties, and applications of micro-and nanostructured thin films. J. Vac. Sci. Technol. 2007, 25, 1317-1336. [CrossRef]

9. Valet, T.; Fert, A. Theory of the perpendicular magnetoresistance in magnetic multilayers. Phys. Rev. B 1993, 48, 7099-7113. [CrossRef]

10. Ohta, T.; Bostwick, A.A.; McChesney, J.L.; Seyller, T.; Horn, K.; Rotenberg, E. Interlayer interaction and electronic screening in multilayer graphene investigated with angle-resolved photoemission spectroscopy. Phys. Rev. Lett. 2007, 98, 206802-206804. [CrossRef]

11. Choi, K.; Park, S.H.; Song, Y.M.; Lee, Y.T.; Hwangbo, C.K.; Yang, H.; Lee, H.S. Nano-tailoring the surface structure for the monolithic high-performance antireflection polymer film. Adv. Mater. 2010, 22, 3713-3718. [CrossRef]

12. Spillman, W.B., Jr.; Sirkis, J.S.; Garnider, P.T. Smart materials and structures: What are they? Smart Mater. Struct. 1996, 5, 247-254. [CrossRef]

13. Arico, A.S.; Bruce, P.; Scrosati, B.; Tarascon, J.M.; van Schalkwijk, W. Nanostructured materials for advanced energy conversion and storage devices. Nat. Mater. 2005, 4, 366-377. [CrossRef]

14. Grier, D.G. A revolution in optical manipulation. Nature 2003, 424, 810-816. [CrossRef]

15. Brett, M.J.; Hawkeye, M.M. New materials at a glance. Science 2008, 319, 1192-1193. [CrossRef]

16. Saladuhkin, I.; Abadias, G.; Uglov, V.; Zlotski, S.; Janse van Vuuren, A.; Herman O'Connell, J. Structural properties and oxidation resistance of $\mathrm{ZrN} / \mathrm{SiN}_{\mathrm{x}}, \mathrm{CrN} / \mathrm{SiN}_{\mathrm{x}}$ and $\mathrm{AlN} / \mathrm{SiN} \mathrm{x}_{\mathrm{x}}$ multilayered films deposited by magnetron sputtering technique. Coatings 2020, 10, 149. [CrossRef]

17. Liang, H.; Geng, X.; Li, W.; Panepinto, A.; Thiry, D.; Chen, M.; Snyders, R. Experimental and modeling study of the fabrication of $\mathrm{Mg}$ nano-sculptured films by magnetron sputtering combined with glancing angle deposition. Coatings 2019, 9, 361. [CrossRef]

18. Proença, M.; Rodrigues, M.S.; Borges, J.; Vaz, F. Gas sensing with nanoplasmonic thin films composed of nanoparticles (Au, Ag) dispersed in CuO matrix. Coatings 2019, 9, 337. [CrossRef]

19. Cougnon, F.; Depla, D. The Seebeck coefficients of sputter deposited metallic thin films: The role of process conditions. Coatings 2019, 9, 299. [CrossRef]

20. Achenbach, J.O.; Mraz, S.; Primetzhofer, D.; Schneider, J.M. Correlative experimental and theoretical investigation of the angle-resolved composition evolution of thin films sputtered from a compound $\mathrm{Mo}_{2} \mathrm{BC}$ target. Coatings 2019, 9, 206. [CrossRef]

21. Tuan, T.T.A.; Kuo, D.H.; Cao, P.T.; Nguyen, V.S.; Pham, Q.P.; Nghi, V.K.; Tran, N.P.L. Electrical characterization of RF reactive sputtered $p-\mathrm{Mg}-\mathrm{In}_{x} \mathrm{Ga}_{1-x} \mathrm{~N} / \mathrm{n}$-Si hetero-junction diodes without using buffer layer. Coatings 2019, 9, 699. [CrossRef]

22. Tuan, T.T.A.; Kuo, D.H.; Cao, P.T.; Pham, Q.P.; Nghi, V.K.; Tran, N.P.L. Electrical and structural properties of all-sputtered $\mathrm{Al} / \mathrm{SiO}_{2} / \mathrm{p}-\mathrm{GaN}$ MOS Schottky diode. Coatings 2019, 9, 685. [CrossRef]

23. Thao, C.P.; Kuo, D.H.; Tuan, T.T.A.; Tuan, K.A.; Vu, N.H.; Na, T.T.V.S.; Nhut, K.V.; Sau, N.V. The effect of RF sputtering conditions on the physical characteristics of deposited GeGaN thin film. Coatings 2019, 9, 645. [CrossRef] 
24. Gago, R.; Prucnal, S.; Hübner, R.; Munnik, F.; Esteban-Mendoza, D.; Jiménez, I.; Palomares, J. Phase selectivity in $\mathrm{Cr}$ and $\mathrm{N}$ co-doped $\mathrm{TiO}_{2}$ films by modulated sputter growth and post-deposition flash-lamp-annealing. Coatings 2019, 9, 448. [CrossRef]

25. Eadi, S.B.; Song, H.S.; Song, H.D.; Oh, J.; Lee, H.D. Nickel film deposition with varying RF power for the reduction of contact resistance in NiSi. Coatings 2019, 9, 349. [CrossRef]

26. Achour, A.; Islam, M.; Ahmad, I.; Saeed, K.; Solaymani, S. Electrochemical stability enhancement in reactive magnetron sputtered VN films upon annealing treatment. Coatings 2019, 9, 72. [CrossRef]

(C) 2020 by the authors. Licensee MDPI, Basel, Switzerland. This article is an open access article distributed under the terms and conditions of the Creative Commons Attribution (CC BY) license (http://creativecommons.org/licenses/by/4.0/). 

Article

\title{
Electrochemical Stability Enhancement in Reactive Magnetron Sputtered VN Films upon Annealing Treatment
}

\author{
Amine Achour ${ }^{1}$, Mohammad Islam ${ }^{2, *}$, Iftikhar Ahmad ${ }^{2}$, Khalid Saeed $^{3}$ \\ and Shahram Solaymani ${ }^{4}$ \\ 1 LISE Laboratory, Research Centre in Physics of Matter and Radiation (PMR), University of Namur, \\ B-5000 Namur, Belgium; a_aminph@yahoo.fr \\ 2 Center of Excellence for Research in Engineering Materials, Deanship of Scientific Research, \\ King Saud University, P.O. Box 800, Riyadh 11421, Saudi Arabia; ifahmad@ksu.edu.sa \\ 3 Department of Mechanical Engineering, King Saud University, P.O. Box 800, Riyadh 11421, Saudi Arabia; \\ khaliduetp@gmail.com \\ 4 Young Researchers and Elite Club, West Tehran Branch, Islamic Azad University, Tehran, Iran; \\ shahram22s2000@yahoo.com \\ * Correspondence: mohammad.islam@gmail.com or miqureshi@ksu.edu.sa; Tel.: +966-54-452-3909
}

Received: 19 December 2018; Accepted: 23 January 2019; Published: 25 January 2019

\begin{abstract}
Vanadium nitride (VN) thin films were produced via direct-current reactive magnetron sputtering technique followed by vacuum annealing. The treatment was carried out at different temperatures for any effect on their electrochemical (EC) stability, up to 10,000 charge-discharge cycles in $0.5 \mathrm{M} \mathrm{K}_{2} \mathrm{SO}_{4}$ solution. The film surface chemistry was investigated by using X-ray photoelectron spectroscope (XPS) and cyclic voltammetry (CV) techniques. For the as-deposited film, the oxide layer formed on the $\mathrm{VN}$ surface was unstable upon $\mathrm{K}_{2} \mathrm{SO}_{4}$ immersion treatment, along with $\sim 23 \%$ reduction in the EC capacitance. Vacuum annealing under optimized conditions, however, made the oxide layer stable with almost no capacitance loss upon cycling for up to 10,000 cycles. Annealing treatment of the $\mathrm{VN}$ films makes them a potential candidate for long-term use in electrochemical capacitors.
\end{abstract}

Keywords: VN films; vacuum annealing; electrochemical capacitor; XPS; cyclic voltammetry

\section{Introduction}

Owing to their high density and melting point, superior hardness, excellent electronic conductivity and high specific capacitance, vanadium nitride $(\mathrm{VN})$ thin films offer strong potential for application in electrochemical capacitors (ECs) [1,2]. In this context, the hybrid nanostructures of VN films and carbon nanotubes have been reported to exhibit high volume capacitance, volume energy and power density [3]. Also, $\mathrm{VN}$ in nanocrystalline form demonstrated gravimetric capacitance of $\sim 1300 \mathrm{~F} \cdot \mathrm{g}^{-1}$, due to successive, fast, and reversible redox reactions involving surface oxide groups and $\mathrm{OH}^{-}$ions from the electrolyte [4]. One of the major disadvantages associated with the use of VN, however, is its susceptibility to degradation that inhibits its practical application. It has been shown that cycling VN in $\mathrm{KOH}$ electrolyte leads to the degradation of the surface oxide layers that form at the surface of $\mathrm{VN}$, and thus the capacitance decay over cycling [4]. Several factors, including film attributes such as crystallite size, morphology, surface oxide layer, etc., and EC test conditions; material loading, electrolyte concentration, and potential window, to name a few, influence the EC performance [5].

Both pure and nanocomposite VN films have been extensively explored for both structural $[6,7]$ and functional applications [8-12]. The VN films exhibit pseudocapacitive behaviors through electric double-layer formation in the presence of $\mathrm{OH}^{-}$ions. Using $\mathrm{N}$-doped carbon nanosheets/VN nanoparticles hybrid composition as the electrode, high specific capacitance with about $60 \%$ retention 
after 5000 cycles was reported [11]. Another study reported a reduction in areal capacitance by $~ 80 \%$ within first 100 cycles, when tested for 1000 cycles in $1 \mathrm{M} \mathrm{KOH}$ solution [2]. Although cycle life stability is generally assessed in $\mathrm{KOH}$ electrolyte solution, it is also performed in mild $\mathrm{K}_{2} \mathrm{SO}_{4}$ solution due to the relatively slow rate of decay in the EC capacitance value $[13,14]$. We recently reported the electrochemical properties of VN/CNT hybrid nanostructures for micro-capacitors, using potassium sulphate $\left(\mathrm{K}_{2} \mathrm{SO}_{4}\right)$ electrolyte [13]. Furthermore, we suggested the preservation of the surface oxide layer for enhanced VN film stability during cycling. In this work, we demonstrate from X-ray photoelectron spectroscope (XPS) analysis that VN film annealing under certain conditions can preserve the surface oxide layer, thus enhancing the cycling life stability in $0.5 \mathrm{M} \mathrm{K}_{2} \mathrm{SO}_{4}$ electrolyte. Such finding have implications in the design of stable VN thin film based materials for use in ECs.

\section{Experimental Procedure}

Direct-current (DC) plasma reactive magnetron sputtering technique was employed to produce VN films over silicon (100) substrates. The system consisted of a magnetron sputtering gun in a stainless steel chamber in which a base pressure of $<10^{-5} \mathrm{~Pa}$ was obtained using a turbo-molecular pump. Pure argon and nitrogen with $99.99 \%$ purity were used as sputtering and reactive gases, respectively. The target was a vanadium metal of $\geq 99.9 \%$ purity. The reactive sputtering was carried out without intentional substrate heating at a pressure of $0.32 \mathrm{~Pa}$. The total gas flux rate during deposition was maintained at a constant value of $40 \mathrm{sccm}$, while the flow rate for reactive $\mathrm{N}_{2}$ gas was fixed at 35\%. The power density and deposition time were kept at $12.7 \mathrm{~W} / \mathrm{cm}^{2}$ and $3 \mathrm{hr}$, respectively. From these deposition conditions, the VN films with an average thickness value of $\sim 690 \mathrm{~nm}$ were obtained, as estimated from scanning electron microscope (SEM) examination of the film cross-sections. The as-deposited VN film is referred to as $\mathrm{V}_{\mathrm{o}}$.

The films were then annealed in the same chamber at different temperatures for $2 \mathrm{~h}$. The pressure inside the chamber during annealing was of the order of $5.5 \times 10^{-3} \mathrm{~Pa}$. Due to low pressure during annealing, excessive oxidation of the films may be ruled out. The samples annealed at 400, 600, and $800{ }^{\circ} \mathrm{C}$ temperature were designated as $\mathrm{V}_{1}, \mathrm{~V}_{2}$, and $\mathrm{V}_{3}$, respectively.

The electrochemical measurements were performed in $0.5 \mathrm{M} \mathrm{K}_{2} \mathrm{SO}_{4}$ (Alfa Aesar, Ward Hill, MA, USA, 99.99\%) electrolyte solution. A conventional cell with 3-electrode configuration was used in a VMP 3 multi potentiostat galvanostat (BioLogic, Seyssinet-Pariset, France) that was coupled with the EC-Lab software ${ }^{\circledR}$ V11.10.

The samples were examined under an SEM (JSM7600F; JEOL, Tokyo, Japan) by operating at $5 \mathrm{kV}$ accelerating voltage and $4.5 \mathrm{~mm}$ working distance. The X-ray diffraction (XRD) patterns were obtained from Siemens D5000 diffractometer (Siemens, Berlin, Germany) with Bragg Brentano configuration and monochromatic $\mathrm{CuK} \alpha 1$ radiation. The film surface chemistry was investigated ex situ using an X-ray photoelectron spectroscope (XPS) (Kratos Axis Ultra, Kratos Analytical Ltd, Manchester, UK). The $\mathrm{Al} \mathrm{K} \alpha$ radiation $(1486.6 \mathrm{eV}$ ) at $20 \mathrm{eV}$ pass energy and $0.9 \mathrm{eV}$ energy resolution was employed to record high-resolution spectra. As a reference, the C $1 \mathrm{~s}$ line of $284.4 \mathrm{eV}$ was used for any correction in the shift in binding energies. The XPS spectra in the V 2p core-level regions were analyzed through a peak-fitting procedure, using a Shirley background.

\section{Results and Discussion}

\subsection{Morphology and Composition}

The SEM microstructures of the as-deposited VN electrode surface and the cross-section are shown in Figure 1. As evident in Figure 1a, the film surface was observed to be comprised of nanostructured grains with a pyramid-like morphology and an average grain size of $\sim 37 \mathrm{~nm}$. Due to this specific granular morphology, the film appeared to exhibit a very high surface area, which could cause an enhancement in the specific capacitance. Also, the high film surface roughness (not measured quantitatively) implied the presence of surface pores, as evident from a few dark, depressed spots 
between grains. The porosity level is nevertheless very low, and is believed to be at the film surface only. Microstructural examination of the film cross-section revealed dense, columnar growth with very little porosity. From the cross-sectional microstructure (Figure 1b), the film thickness was estimated to be $\sim 690 \mathrm{~nm}$, with a corresponding growth rate of $230 \mathrm{~nm} / \mathrm{hr}$. Although not shown here, the $\mathrm{V}_{1}, \mathrm{~V}_{2}$, and $V_{3}$ films did not undergo any noticeable change in surface morphology upon vacuum thermal annealing at temperatures of 400 to $800{ }^{\circ} \mathrm{C}$.

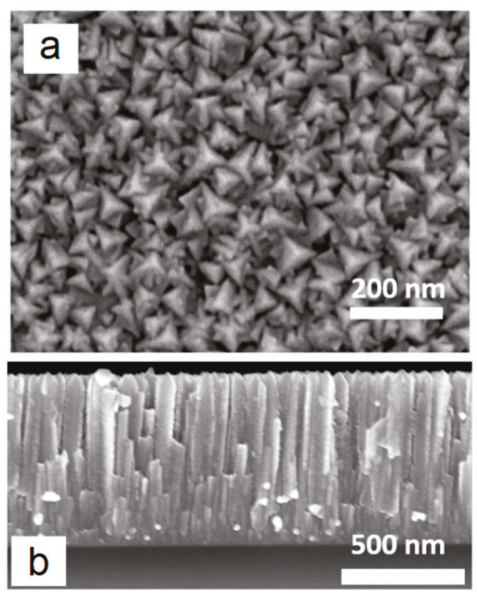

Figure 1. SEM micrographs of the as-prepared vanadium nitride (VN) film: (a) Surface microstructure, and (b) Cross-sectional view.

The XRD patterns of the as-deposited and vacuum annealed VN films are presented in Figure 2. All the samples exhibited one peak located at $\sim 37.8^{\circ}$ that was indexed to be (111) plane of the face-centered cubic VN (JCPDS No. 35-0768) [15]. The peak is broad, indicative of the small size of the individual crystallites in the deposited film. Upon thermal treatment, a small shift towards a greater diffraction angle was noticed, which may be attributed to stress relaxation, with consequent reduction in the lattice constant. The fcc $\delta$-VN phase formation under the prevailing conditions of power density $\left(12.7 \mathrm{~W} \cdot \mathrm{cm}^{-2}\right)$ and nitrogen gas flow $(14 \mathrm{sccm})$ as well as the resulting film growth rate $\left(3.83 \mathrm{~nm} \cdot \mathrm{min}^{-1}\right)$ confirm the findings reported earlier $[16,17]$.

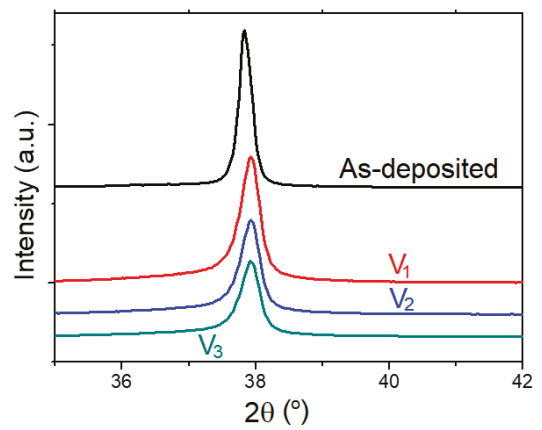

Figure 2. XRD patterns of the as-deposited and vacuum-annealed VN films. 


\subsection{Cyclic Voltammetry and XPS Analysis of as-Deposited VN Film}

For the as-deposited $\mathrm{VN}$ film $\left(\mathrm{V}_{\mathrm{o}}\right)$, cyclic voltammetry experiments were performed at a $200 \mathrm{mV} \cdot \mathrm{s}^{-1}$ scan rate. The cyclic voltammograms of the VN films after 3 and 10,000 consecutive cycles are showcased in Figure 3. Upon repeated charge-discharge cycling, a decay in the electrochemical capacitance by $\sim 23 \%$ was observed. It is noteworthy that the electrochemical treatment did not induce any modification in the film surface morphology or structure after cycling.

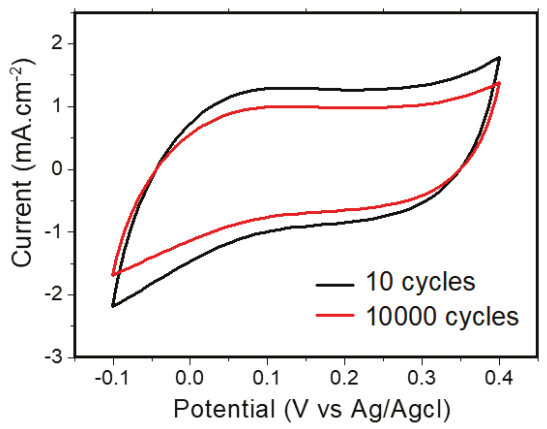

Figure 3. Cycling voltammograms of the as-deposited $\mathrm{VN}$ film in $\mathrm{K}_{2} \mathrm{SO}_{4}$ solution at a $200 \mathrm{mV} \cdot \mathrm{s}^{-1}$ scan rate after 3 and 10,000 cycles.

XPS analysis was performed to determine any changes in film surface chemistry before and after EC cycling. For the as-deposited VN film (no thermal treatment), the chemical nature of the film surface is showcased in Figure 4 through V 2p and N 1s high resolution XPS spectra before and after EC cycling. The $\mathrm{V} 2 \mathrm{p}_{3 / 2}$ spectral regime revealed presence of four peaks at binding energies (BE) of 513.4, $514.2,515.5$, and $517.1 \mathrm{eV}$. While the first peak is associated with $\mathrm{VN}$, the latter three may be assigned to $\mathrm{V}_{2} \mathrm{O}_{3}, \mathrm{VO}_{2}$ and $\mathrm{V}_{2} \mathrm{O}_{5}$ compositions, respectively $[18,19]$. It is noteworthy that, like most transition metal nitrides, surface oxidation of the $\mathrm{VN}$ film occurred upon air exposure and aging. It was observed that in the V 2p spectrum (Figure 4a), the intensity of the $\mathrm{V}_{2} \mathrm{O}_{5}$ peak was reduced after cycling. On the other hand, the peak intensity for $\mathrm{VO}_{x} \mathrm{~N}_{y}$ remained unchanged after cycling, as revealed by high resolution spectra of the $\mathrm{N}$ 1s peaks (Figure $4 \mathrm{~b}$ ). The electrochemical charge-discharge cycling of the as-deposited VN film, therefore, causes deterioration of the oxide layer over the VN film surface, thus inducing a drop in the capacitance, as noticed in the cyclic voltammetry results (Figure 3).
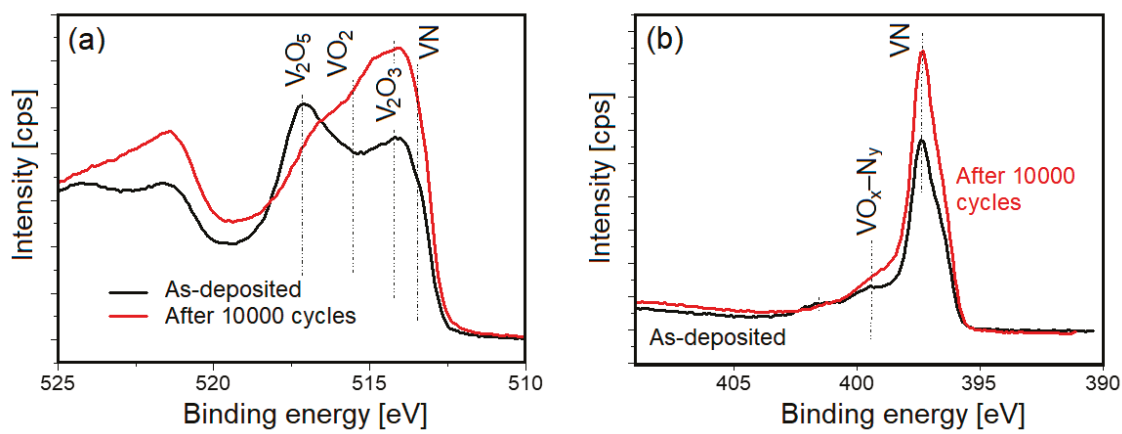

Figure 4. X-ray photoelectron spectroscope (XPS) data of the as-deposited VN film before and after 10,000 charge-discharge cycles: (a) Core level V 2p spectra, and (b) Core level N 1s spectra. 


\subsection{Effect of Vacuum Annealing: XPS Analysis}

The high resolution XPS V 2p spectra of the as-deposited as well as thermally treated VN films are represented in Figure 5. Before the EC charge/discharge cycling, the surface chemistry of the vacuum annealed VN films was different from that of as-produced film, in the sense that 600 and $800{ }^{\circ} \mathrm{C}$ thermal treatments promoted a slightly higher degree of surface oxide layer formation. This may be attributed to the partial oxidation of the film surface at high temperatures, even under vacuum [20]. Among the vacuum-annealed $\mathrm{VN}$ films, only $\mathrm{V}_{1}$ sample (annealed at $400{ }^{\circ} \mathrm{C}$ ) was noticed to undergo oxide degradation after 10,000 EC cycles, as evident from comparison of the XPS spectra (Figure 5a). Quite interestingly, however, the oxide content on the $\mathrm{VN}$ film surfaces in the case of $\mathrm{V}_{2}$ and $\mathrm{V}_{3}$ films (annealed at 600 and $800^{\circ} \mathrm{C}$ ) did not decrease after prolonged cycling, as demonstrated in Figure 5b,c.
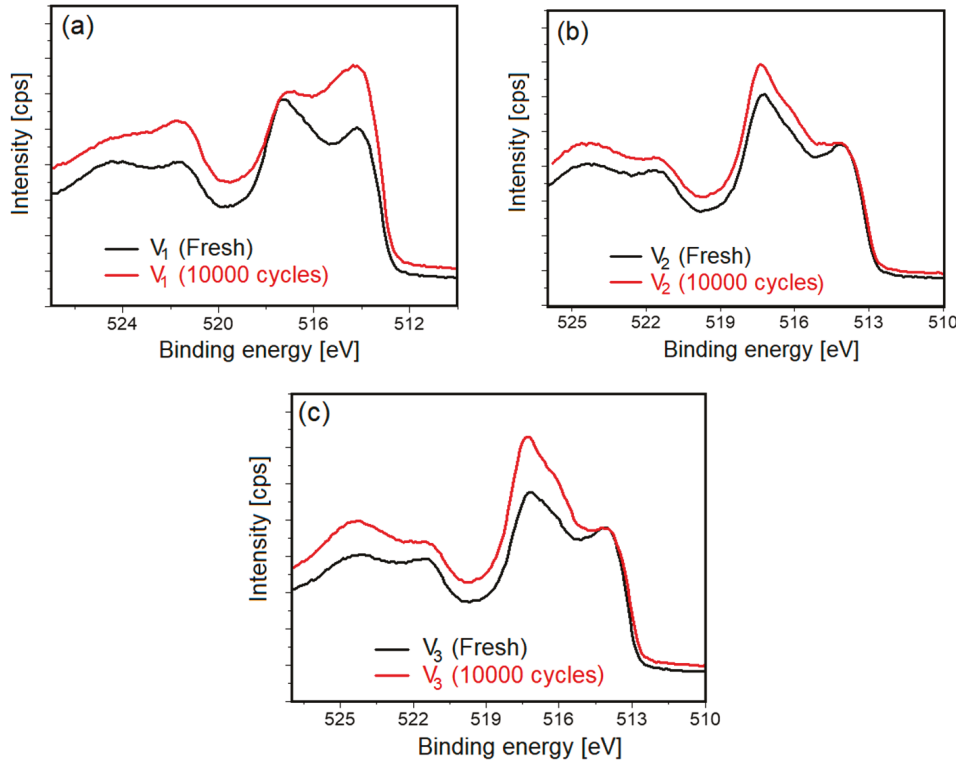

Figure 5. Comparison of the XPS core level V 2p spectra for the annealed VN films initially and after 10000 EC cycles: (a) $V_{1}$ sample, (b) $V_{2}$ sample, and (c) $V_{3}$ sample.

After EC charge/discharge tests for up to 10,000 cycles, the areal capacitance was plotted against the number of cycles, as shown in Figure $6 \mathrm{a}$. For the $\mathrm{V}_{2}$ and $\mathrm{V}_{3}$ samples i.e., the $\mathrm{VN}$ films annealed at 600 and $800{ }^{\circ} \mathrm{C}$, there was no deterioration in the areal capacitance level. Moreover, the specific capacitance was observed to be greater for the vacuum-annealed films, presumably because of an increase in the amount of oxide layer at the VN film surface. These findings indicate that vacuum annealing treatment leads to stabilization of the oxide surface layer. Factors such as formation of a thicker oxide layer, or crystallization of the oxide surface layer or both, might have been responsible for this behavior. The XPS V 2p core level spectra of the annealed VN films were recorded and deconvoluted to investigate the bonding characteristics of vanadium with oxygen and nitrogen, as presented in Figure $6 \mathrm{~b}-\mathrm{d}$. The atomic percent of vanadium, bonded as $\mathrm{V}_{2} \mathrm{O}_{3}, \mathrm{VO}_{2}$, and $\mathrm{V}_{2} \mathrm{O}_{5}$ compositions, are given as an inset in each case. Among the three samples examined, the presence of $\mathrm{V}_{2} \mathrm{O}_{5}$ and $\mathrm{V}_{2} \mathrm{O}_{3}$ phases was found to be maximized in case of sample $\mathrm{V}_{2}$ (vacuum-annealed at $600{ }^{\circ} \mathrm{C}$ ). Although the relative content of the $\mathrm{VO}_{2}$ phase remained almost the same in all the films, there was an associated reduction in the $\mathrm{VN}$ phase content upon vacuum annealing. These observations signify the importance of annealing conditions towards any improvement in the electrochemical properties. In addition, the correlation between the capacitance improvement and the surface chemistry of the VN 
films may offer an insight into the influence of the $\mathrm{V}_{y} \mathrm{O}_{x}$ composition on the capacitance behavior of the VN electrodes.
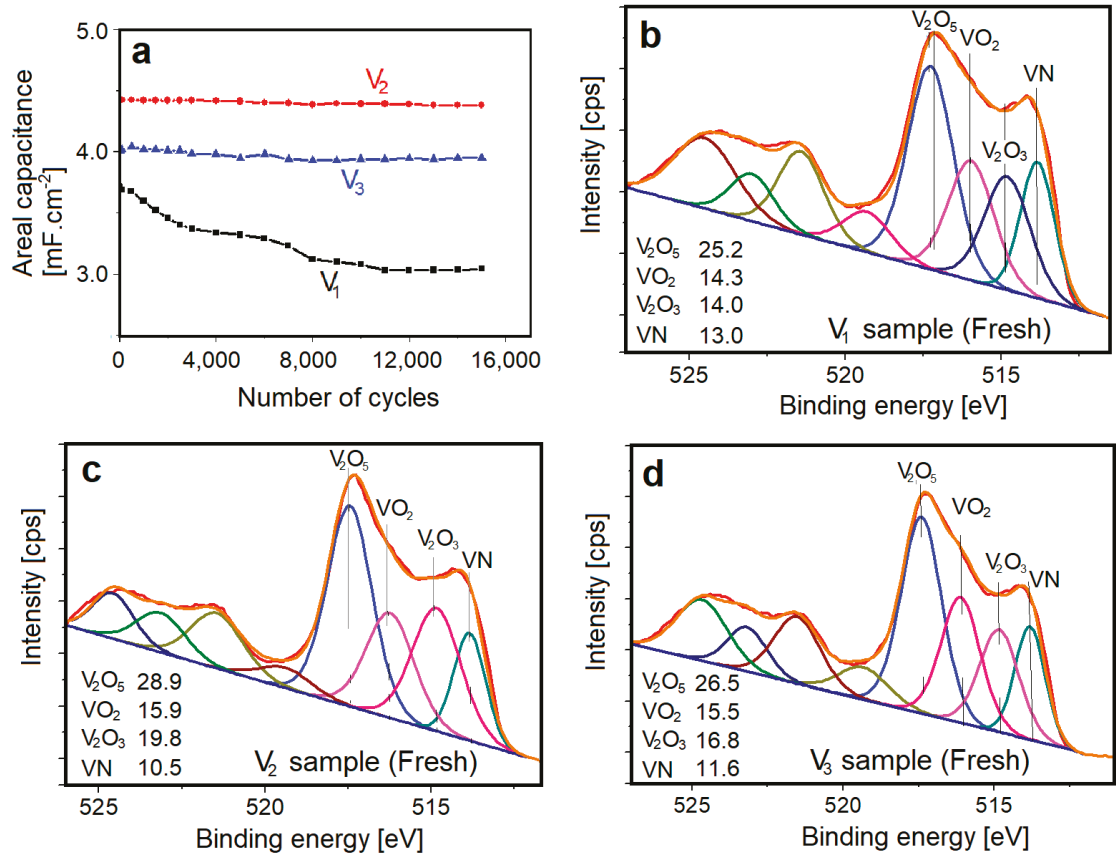

Figure 6. Cycling stability of the annealed VN film at $200 \mathrm{mV} \cdot \mathrm{s}^{-1}$ scan rate, and (b-d) XPS V 2p core level deconvoluted spectra of the annealed VN films: (b) $V_{1}$ sample, (c) $V_{2}$ sample, and (d) $V_{3}$ sample.

\section{Conclusions}

Nanocrystalline VN films were produced via DC-plasma reactive magnetron sputtering followed by thermal treatment under a vacuum at 400,600 , and $800{ }^{\circ} \mathrm{C}$. For the as-deposited and $400{ }^{\circ} \mathrm{C}$ annealed VN films, the topmost surface oxide layer was found to degrade upon treatment in $\mathrm{K}_{2} \mathrm{SO}_{4}$ electrolyte solution. This was confirmed from XPS analysis besides a $\sim 23 \%$ capacitance loss in the case of the as-deposited VN film after 10,000 cycles. Vacuum annealing of the VN films at 600 and $800{ }^{\circ} \mathrm{C}$ led to an enhancement in electrochemical cycling stability, with an almost $100 \%$ capacitance retention, even after 10,000 cycles. Such an improvement in EC properties is speculated to originate from VN film crystallization or a thickness increase in the surface oxide layer, or both. From this finding, VN-based electrode materials may be developed with superior VN cycling stability in electrochemical energy devices.

Author Contributions: A.A. and M.I. conceived the idea and designed the experimentation scheme; A.A., K.S., and I.A. performed the experiments; M.I. and S.S. carried out data analysis; A.A. and M.I. prepared the original manuscript draft with input from other co-authors. M.I. was responsible for acquisition of funding for this research.

Funding: The authors would like to extend their sincere appreciation to the Deanship of Scientific Research at King Saud University for its funding of this research through the Research Group No. RGP-283.

Conflicts of Interest: The authors declare no conflict of interest. 


\section{References}

1. Eustache, E.; Frappier, R.; Porto, R.L.; Bouhtiyya, S.; Pierson, J.F.; Brousse, T. Asymmetric electrochemical capacitor microdevice designed with vanadium nitride and nickel oxide thin film electrodes. Electrochem. Commun. 2013, 28, 104-106. [CrossRef]

2. Liu, X.; Lu, H.; He, M.; Jin, K.; Yang, G.; Ni, H.; Zhao, K. Epitaxial growth of vanadium nitride thin films by laser molecule beam epitaxy. Mater. Lett. 2014, 123, 38-40. [CrossRef]

3. Xiao, X.; Peng, X.; Jin, H.; Li, T.; Zhang, C.; Gao, B.; Hu, B.; Huo, K.; Zhou, J. Freestanding mesoporous VN/CNT hybrid electrodes for flexible all-solid-state supercapacitors. Adv. Mater. 2013, 25, 5091-5097. [CrossRef] [PubMed]

4. Choi, D.; Blomgren, G.E.; Kumta, P.N. Fast and reversible surface redox reaction in nanocrystalline vanadium nitride supercapacitors. Adv. Mater. 2006, 18, 1178-1182. [CrossRef]

5. Shu, D.; Lv, C.; Cheng, F.; He, C.; Yang, K.; Nan, J.; Long, L. Enhanced capacitance and rate capability of nanocrystalline VN as electrode materials for supercapacitors. Int. J. Electrochem. Sci. 2013, 8, 1209-1225.

6. Fu, T.; Peng, X.; Wan, C.; Lin, Z.; Chen, X.; Hu, N.; Wang, Z. Molecular dynamics simulation of plasticity in $\mathrm{VN}(001)$ crystals under nanoindentation with a spherical indenter. Appl. Surf. Sci. 2017, 392, 942-949. [CrossRef]

7. Sowa, M.J.; Ju, L.; Kozen, A.C.; Strandwitz, N.C.; Zeng, G.; Babuska, T.F.; Hsain, Z.; Krick, B.A. Plasma-enhanced atomic layer deposition of titanium vanadium nitride. J. Vac. Sci. Technol. A 2018, 36, 06A103. [CrossRef]

8. Yan, Y.; Li, B.; Guo, W.; Pang, H.; Xue, H. Vanadium based materials as electrode materials for high performance supercapacitors. J. Power Sources 2016, 329, 148-169. [CrossRef]

9. Zhu, L.; Li, C.; Ren, W.; Qin, M.; Xu, L. Multifunctional vanadium nitride@N-doped carbon composites for kinetically enhanced lithium-sulfur batteries. New J. Chem. 2018, 42, 5109-5116. [CrossRef]

10. Wu, Y.; Ran, F. Vanadium nitride quantum dot/nitrogen-doped microporous carbon nanofibers electrode for high-performance supercapacitors. J. Power Sources 2017, 344, 1-10. [CrossRef]

11. Tan, Y.; Liu, Y.; Tang, Z.; Wang, Z.; Kong, L.; Kang, L.; Liu, Z.; Ran, F. Concise N-doped carbon nanosheets/vanadium nitride nanoparticles materials via intercalative polymerization for supercapacitors. Sci. Rep. 2018, 8, 2915. [CrossRef] [PubMed]

12. Guo, J.; Zhang, Q.; Sun, J.; Li, C.; Zhao, J.; Zhou, Z.; He, B.; Wang, X.; Man, P.; Li, Q.; et al. Direct growth of vanadium nitride nanosheets on carbon nanotube fibers as novel negative electrodes for high-energy-density wearable fiber-shaped asymmetric supercapacitors. J. Power Sources. 2018, 382, 122-127. [CrossRef]

13. Ouldhamadouche, N.; Achour, A.; Lucio-Porto, R.; Islam, M.; Solaymani, S.; Arman, A.; Ahmadpourian, A.; Achour, H.; Le Brizoual, L.; Djouadi, M.A.; et al. Electrodes based on nano-tree-like vanadium nitride and carbon nanotubes for micro-supercapacitors. J. Mater. Sci. Technol. 2018, 34, 976-982. [CrossRef]

14. Bondarchuk, O.; Morel, A.; Bélanger, D.; Goikolea, E.; Brousse, T.; Mysyk, R. Thin films of pure vanadium nitride: Evidence for anomalous nonfaradaic capacitance. J. Power Sources 2016, 324, 439-446. [CrossRef]

15. Hajihoseini, H.; Kateb, M.; Ingvarsson, S.; Gudmundsson, J.T. Effect of substrate bias on properties of HiPIMS deposited vanadium nitride films. Thin Solid Films 2018, 663, 126-130. [CrossRef]

16. Farges, G.; Beauprez, E.; Degout, D. Preparation and characterization of V-N films deposited by reactive triode magnetron sputtering. Surf. Coat. Technol. 1992, 54-55, 115-120.

17. Hajihoseini, H.; Gudmundsson, J.T. Vanadium and vanadium nitride thin films grown by high power impulse magnetron sputtering. J. Phys. D Appl. Phys. 2017, 50, 505302. [CrossRef]

18. Sun, Y.; Liu, K.; Miao, J.; Wang, Z.; Tian, B.; Zhang, L.; Li, Q.; Fan, S.; Jiang, K. Highly sensitive surface-enhanced Raman scattering substrate made from superaligned carbon nanotubes. Nano Lett. 2010, 10, 1747-1753. [CrossRef] [PubMed]

19. Boukhalfa, S.; Evanoff, K.; Yushin, G. Atomic layer deposition of vanadium oxide on carbon nanotubes for high-power supercapacitor electrodes. Energy Environ. Sci. 2012, 5, 6872-6879. [CrossRef]

20. Glaser, A.; Surnev, S.; Netzer, F.P.; Fateh, N.; Fontalvo, G.A.; Mitterer, C. Oxidation of vanadium nitride and titanium nitride coatings. Surf. Sci. 2007, 601, 1153-1159. [CrossRef]

(C) 2019 by the authors. Licensee MDPI, Basel, Switzerland. This article is an open access article distributed under the terms and conditions of the Creative Commons Attribution (CC BY) license (http:/ / creativecommons.org/licenses/by/4.0/). 

Article

\title{
Correlative Experimental and Theoretical Investigation of the Angle-Resolved Composition Evolution of Thin Films Sputtered from a Compound $\mathrm{Mo}_{2} \mathrm{BC}$ Target
}

\author{
Jan-Ole Achenbach ${ }^{1, *}$, Stanislav Mráz ${ }^{1}$, Daniel Primetzhofer ${ }^{2}$ and Jochen M. Schneider ${ }^{1}$ \\ 1 Materials Chemistry, RWTH Aachen University, Kopernikusstr. 10, 52074 Aachen, Germany; \\ mraz@mch.rwth-aachen.de (S.M.); schneider@mch.rwth-aachen.de (J.M.S.) \\ 2 Department of Physics and Astronomy, Uppsala University, Lägerhyddsvägen 1, 75120 Uppsala, Sweden; \\ daniel.primetzhofer@physics.uu.se \\ * Correspondence: achenbach@mch.rwth-aachen.de; Tel.: +49-241-80-25997
}

Received: 1 March 2019; Accepted: 20 March 2019; Published: 22 March 2019

\begin{abstract}
The angle-resolved composition evolution of Mo-B-C thin films deposited from a $\mathrm{Mo}_{2} \mathrm{BC}$ compound target was investigated experimentally and theoretically. Depositions were carried out by direct current magnetron sputtering (DCMS) in a pressure range from 0.09 to $0.98 \mathrm{~Pa}$ in $\mathrm{Ar}$ and $\mathrm{Kr}$. The substrates were placed at specific angles $\alpha$ with respect to the target normal from 0 to $\pm 67.5^{\circ}$. A model based on TRIDYN and SIMTRA was used to calculate the influence of the sputtering gas on the angular distribution function of the sputtered species at the target, their transport through the gas phase, and film composition. Experimental pressure- and sputtering gas-dependent thin film chemical composition data are in good agreement with simulated angle-resolved film composition data. In Ar, the pressure-induced film composition variations at a particular $\alpha$ are within the error of the EDX measurements. On the contrary, an order of magnitude increase in $\mathrm{Kr}$ pressure results in an increase of the Mo concentration measured at $\alpha=0^{\circ}$ from 36 at. $\%$ to 43 at. $\%$. It is shown that the mass ratio between sputtering gas and sputtered species defines the scattering angle within the collision cascades in the target, as well as for the collisions in the gas phase, which in turn defines the angle- and pressure-dependent film compositions.
\end{abstract}

Keywords: physical vapor deposition; $\mathrm{Mo}_{2} \mathrm{BC}$; Monte Carlo simulation; scattering; density functional theory

\section{Introduction}

$\mathrm{Mo}_{2} \mathrm{BC}$ is classified as a nanolaminated material with an orthorhombic structure [1-3]. It shows a unique combination of mechanical properties, such as an elastic modulus of $470 \mathrm{GPa}$, a ratio of bulk and shear moduli of 1.73, and a positive Cauchy pressure, which are required for hard and wear-resistant coatings with moderate ductility [3,4]. Bolvardi et al. [5] successfully synthesized crystalline $\mathrm{Mo}_{2} \mathrm{BC}$ at $380^{\circ} \mathrm{C}$ by high power pulse magnetron sputtering (HPPMS) [6] compared to a required temperature of $580^{\circ} \mathrm{C}$ during direct current magnetron sputtering (DCMS) [7]. The lower deposition temperature for the synthesis of a crystalline thin film by HPPMS was attributed to a larger adatom mobility induced by ion bombardment during HPPMS.

There are several synthesis approaches for the deposition of compound thin films, such as the utilization of reactive gases [7], co-sputtering from several targets [8,9], or targets with plugs [10]. Likewise, the employment of multi-elemental powder metallurgical composite targets is of great interest from an industrial application point of view due to the enhanced stability and repeatability [11] of non-reactive sputtering compared to reactive sputtering processes. However, it has been shown 
that the chemical composition of thin films deposited from multi-element targets deviates from the target composition, especially in targets with significant mass differences between their constituents, such as TiB [12-14], TiW [15-22], WB [23], SiC [24], MoSi [25,26], VC [27], NbC [28], Cr-Al-C [29,30], $\mathrm{Ti}_{2} \mathrm{AlC}$ [31], $\mathrm{Ti}_{3} \mathrm{SiC}_{2}$ [32,33], and CuZnSnSe [34]. The difference in the chemical film composition was attributed to several mechanisms: (i) the mass and size differences of the target constituents and the respectively associated different angular and energy distribution functions (EDF) of the sputtered species $[12,25,26,29,35]$; (ii) their mean free paths, as well as the energy transfer in collisions with the sputtering gas during transport $[12,13,15,21,29,35]$; and (iii) different sticking coefficients and re-sputtering of the film constituents by backscattered Ar [15-20].

The compositional evolution of binary Ti-B thin films was investigated experimentally and with a Monte Carlo model based on TRIDYN (dynamic transport of ions in matter) and TRIM (transport of ions in matter) codes [12]. It was shown that the Ti/B ratio strongly depends on the gas pressure and target-substrate distance, which in a product is proportional to the number of collisions sputtered species experience within the gas phase. The model was extended to $\mathrm{Cr}-\mathrm{Al}-\mathrm{C}$ thin films-a ternary system [29].

Van Aeken et al. [36] developed a Monte Carlo code SIMTRA for the simulation of sputtered particle trajectories in a gas-phase within a definable 3D setup. Collision modelling by interatomic potentials and thermal motion of background atoms are included within the code.

From the above, it can be learned that the deviation of the chemical composition of a thin film and multi-element target can be controlled by the sputtering pressure and gas type.

Within this work, experimental data were compared to a model based on TRIDYN and SIMTRA utilized for Mo-B-C thin films to understand how the gas phase transport affects the thin film chemical composition in a system with large mass differences of the multi-element target constituents.

\section{Materials and Methods}

\subsection{Experimental Details}

Mo-B-C thin films were deposited in a high vacuum chamber assembled from a DN160 six-way cross. A base pressure of $<1.1 \times 10^{-4}$ Pa was achieved before all depositions with a combination of a rotary-vane (Edwards E2M28, Edwards, Burgess Hill, UK) and a turbomolecular pump (Pfeiffer Vacuum TPU 240, Aßlar, Germany). A self-built magnetron with $\varnothing 90 \mathrm{~mm}$ was placed in the center of the chamber. A $6 \mathrm{~mm}$ thick $\mathrm{Mo}_{2} \mathrm{BC}$ compound target (Plansee Composite Materials GmbH, Lechbruck am See, Germany) with the composition of 54.3 at.\%, 24.2 at.\%, and 21.5 at.\% of Mo, B, and C, respectively, bonded on a $\mathrm{Cu}$ backing-plate, was utilized for the investigations. The target contained a major $\mathrm{Mo}_{2} \mathrm{BC}$ phase with minor $\mathrm{Mo}_{2} \mathrm{C}$ and $\mathrm{MoC}$ phases (Figure 1), as measured by a Bruker D8 Discovery general area detector diffraction system (GADDS, Bruker, Billerica, MA, USA) with $\mathrm{Cu}(\mathrm{K} \alpha)$ radiation at $40 \mathrm{kV}$ and $40 \mathrm{~mA}$ with a constant incident angle of $\omega=15^{\circ}$.

The thin films were deposited for $1 \mathrm{~h}$ onto grounded, not intentionally heated Si (100) substrates with a size of approximately $15 \times 15 \mathrm{~mm}^{2}$ arranged at different angular positions with respect to the target normal of $\alpha \in\left\{0^{\circ}, \pm 22.5^{\circ}, \pm 45^{\circ}\right.$ and $\left.\pm 67.5^{\circ}\right\}$ (Figure 2). The target-substrate distance was kept constant at $70 \mathrm{~mm}$ with respect to the target center point. The DC power of $100 \mathrm{~W}$ was applied by an ADL $1.5 \mathrm{~kW}$ DC power supply (ADL Analoge und Digitale Leistungselektronik GmbH, Darmstadt, Germany). The Ar and $\mathrm{Kr}$ pressures utilized in the depositions are summarized in Table 1. 


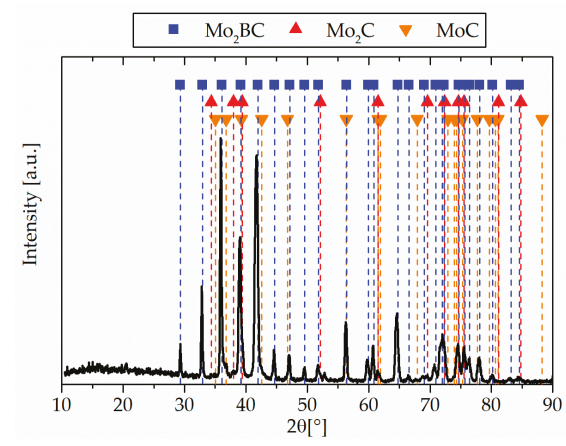

Figure 1. XRD pattern of the powder-metallurgically manufactured $\mathrm{Mo}_{2} \mathrm{BC}$ compound target. Small phase fractions of $\mathrm{Mo}_{2} \mathrm{C}$ and $\mathrm{MoC}$ were detected.

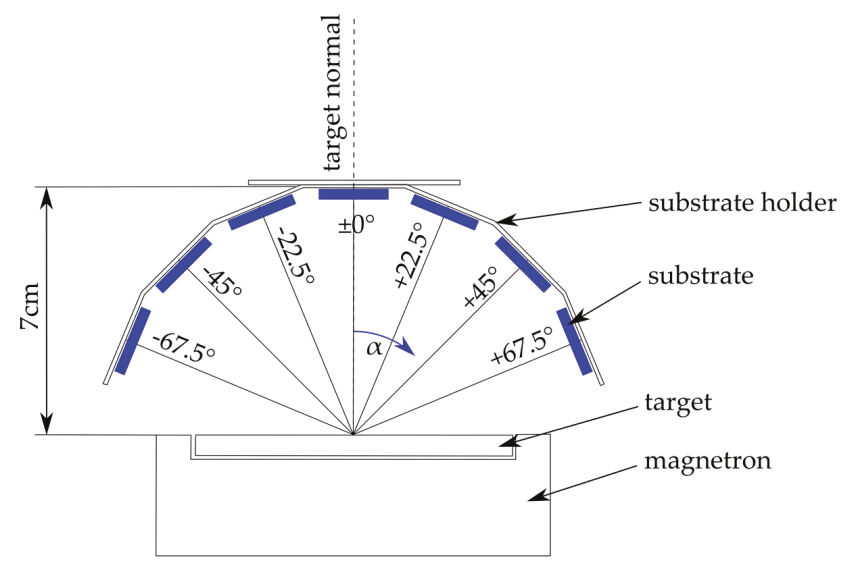

Figure 2. Experimental setup with seven substrates positioned at $\alpha \in\left\{0^{\circ}, \pm 22.5^{\circ}, \pm 45^{\circ}\right.$ and $\left.\pm 67.5^{\circ}\right\}$ angle arrangement with respect to the target normal and a target-substrate distance of approximately $70 \mathrm{~mm}$.

Table 1. Ar and $\mathrm{Kr}$ gas pressures and measured target voltages which correspond to impinging ion energies for $\mathrm{Ar}^{+}$and $\mathrm{Kr}^{+}$ions.

\begin{tabular}{cccc}
\hline \multicolumn{2}{c}{ Argon } & \multicolumn{2}{c}{ Krypton } \\
\hline Pressure (Pa) & Voltage (V) & Pressure (Pa) & Voltage (V) \\
\hline 0.09 & 401 & 0.09 & 441 \\
0.27 & 344 & 0.26 & 423 \\
0.46 & 328 & 0.45 & 421 \\
0.66 & 324 & 0.64 & 418 \\
0.98 & 314 & 0.96 & 403 \\
\hline
\end{tabular}

The chemical composition of the deposited films was measured by energy dispersive X-ray spectroscopy (EDX) attached to a JEOL JSM-6480 scanning electron microscope (SEM, JEOL, Tokyo, Japan). The electron gun of the SEM was operated at an acceleration voltage of $5 \mathrm{kV}$. Each sample was measured 10 times. The statistical uncertainty associated with this EDX quantification of Mo, B, and C was less than or equal to $5 \%$ relative deviation. To overcome the unknown systematic uncertainty for light elements in EDX, the samples deposited at 0.66 Pa Ar with $\alpha=0^{\circ},-22.5^{\circ},-45^{\circ}$, and $-67.5^{\circ}$ were quantified by time-of-flight elastic recoil detection analysis (ToF-ERDA) and used as a standard for the 
respective positions. The statistical uncertainty for all ToF-ERDA was $<0.4 \%$ absolute. In ToF-ERDA, the relative systematic uncertainties in the specific energy loss of the constituents and primary ions of the target are assumed to range from $5 \%$ to $10 \%$. Hence, the lower bound of the total measurement uncertainty for the EDX analysis with ToF-ERDA quantified standards ranges from $7 \%$ to $11 \%$.

\subsection{Simulation Details}

The angular-resolved chemical composition of the thin films was simulated with a Monte Carlo model based on TRIDYN [37,38] and SIMTRA [36] for the sputtering process and the gas phase transport, respectively.

\subsubsection{TRIDYN}

The impinging ion energies of $\mathrm{Ar}^{+}$and $\mathrm{Kr}^{+}$ions in the TRIDYN simulation were set according to the experimentally measured target voltages (Table 1). To address the dependence of the surface binding energy from the surface chemistry, a matrix model was introduced [38] and modified [29] for a system containing three elements, as presented in Equation (1), where $\mathrm{SBE}_{i}$ is the surface binding energy of the $i$-th target element at a given target concentration $c, c_{i}$ is the concentration of the $i$-th target element, and $\mathrm{SBV}_{i-j}$ is the surface binding potential of the $i$-th and $j$-th elements. $\mathrm{SBV}_{i-j}$ are assumed to be constant. Calculated angular distribution functions (ADF) and energy distribution functions (EDF) of the sputtered species are utilized in SIMTRA.

$$
\left(\begin{array}{c}
\mathrm{SBE}_{\mathrm{Mo}} \\
\mathrm{SBE}_{\mathrm{B}} \\
\mathrm{SBE}_{\mathrm{C}}
\end{array}\right)=\left(\begin{array}{ccc}
\mathrm{SBV}_{\mathrm{Mo}-\mathrm{Mo}} & \mathrm{SBV}_{\mathrm{Mo}-\mathrm{B}} & \mathrm{SBV}_{\mathrm{Mo}-\mathrm{C}} \\
\mathrm{SBV}_{\mathrm{B}-\mathrm{Mo}} & \mathrm{SBV}_{\mathrm{B}-\mathrm{B}} & \mathrm{SBV}_{\mathrm{B}-\mathrm{C}} \\
\mathrm{SBV}_{\mathrm{C}-\mathrm{Mo}} & \mathrm{SBV}_{\mathrm{C}-\mathrm{B}} & \mathrm{SBV}_{\mathrm{C}-\mathrm{C}}
\end{array}\right)\left(\begin{array}{c}
\mathrm{C}_{\mathrm{Mo}} \\
\mathrm{C}_{\mathrm{B}} \\
\mathrm{C}_{\mathrm{C}}
\end{array}\right)
$$

For the determination of the surface binding potentials, an approach based on the energy conservation law $[29,38]$ was used and will in the following be called the energy conservation law approach. In addition, a DFT ab initio-based approach has been employed.

\subsubsection{Energy Conservation Law Approach}

The surface binding potential of pure elements $\mathrm{SBV}_{i-i}$ is assumed to be equal to the enthalpy of sublimation $\Delta_{\text {sub }} H_{i}$. The surface binding potential of the atom pairs $\mathrm{SBV}_{i-j}$ is calculated using Equation (2), where $\Delta_{\mathrm{f}} H_{\mathrm{Mo}_{\mathrm{n}} \mathrm{B}_{\mathrm{m}} \mathrm{C}_{\mathrm{o}}}$ is the enthalpy of formation of the ternary compound and $a$ and $b$ are the stoichiometric factors of the elements $i$ and $j$.

$$
\operatorname{SBV}_{i-j}=\frac{1}{2}\left(\Delta_{\text {sub }} H_{i}+\Delta_{\text {sub }} H_{j}\right)-\frac{1}{3} \frac{\mathrm{n}+\mathrm{m}+\mathrm{o}}{2 a b} \Delta_{\mathrm{f}} H_{\mathrm{Mon}_{\mathrm{n}} \mathrm{B}_{\mathrm{m}} \mathrm{C}_{\mathrm{o}}}
$$

The energy of formation per formula unit (f.u.) of $\Delta_{\mathrm{f}} \mathrm{H}_{\mathrm{Mo}_{2} \mathrm{BC}}=-1.132 \frac{\mathrm{eV}}{\mathrm{f} u .}$ used in the simulations was calculated by Bolvardi et al. [4]. The enthalpies of sublimation of $6.83,5.73$, and $7.51 \mathrm{eV}$ for Mo, B, and $\mathrm{C}$ are given in the elements.dat file of TRIDYN, respectively. In addition, enthalpies of sublimation of 6.81, 5.75, and $7.37 \mathrm{eV}$ for Mo, B, and C, respectively, can be found in [39].

\subsubsection{Ab Initio Approach}

In addition to the TRIDYN approach, an ab initio approach based on DFT was used for the determination of the respective surface binding potentials. DFT calculations were implemented within the Vienna ab initio simulation package (VASP) [40,41]. Perdew-Burke-Ernzerhof (PBE) adjusted generalized gradient approximation (GGA) [42] was used for all calculations with projector augmented wave potential [43]. In addition, the tetrahedron method for total energy using Blöchl-corrections [44] and the reciprocal space integration using the Monkhorst-Pack scheme [45] were applied. The utilized $k$-point grid was $4 \times 4 \times 4$ for the (100) and (001) surfaces and $6 \times 2 \times 6$ for the (010) surface. The cut-off energy was set to $500 \mathrm{eV}$ with an electronic relaxation convergence of $0.01 \mathrm{meV}$. 
Considering the matrix model presented in Equation (1), the energy required to remove atoms of specific surfaces with different chemical compositions needs to be calculated. (100) and (001) surfaces, as well as different surface terminations of the (010) surface, are considered in the calculation and are illustrated in Figure 3. Subsequently, atoms are removed from the surface, creating a vacancy. The change in energy is considered to be the surface binding potential of the atom within the respective surface, as shown in Equation (3). $E_{i}$ is the energy of the atom $i$ after being removed from the surface, $E_{\mathrm{vac}, i}^{\text {sure, } j}$ is the energy of surface $j$ with the vacancy of atom $i$, and $E^{\text {surface } j}$ is the energy of surface $j$ without a defect. Within DFT, the surfaces were simulated by a vacuum layer on top of the unit cell with the height of approximately $10 \AA$ for (100) and (001) and $17 \AA$ for (010) surfaces. Calculated SBVs for both approaches are presented in Equations (4) and (5).

$$
\begin{gathered}
\mathrm{SBV}_{i-j}=E_{i}+E_{\text {vac, }, i}^{\text {sura }, j}-E^{\text {surface }, j} \\
\mathrm{SBV}_{\text {energy conservation law }}=\left(\begin{array}{lll}
6.83 & 6.66 & 7.50 \\
6.66 & 5.73 & 7.32 \\
7.50 & 7.32 & 7.41
\end{array}\right) \mathrm{eV} \\
\mathrm{SBV}_{\mathrm{ab} \text { initio }}=\left(\begin{array}{ccc}
7.25 & 7.33 & 9.46 \\
7.19 & 6.98 & 9.71 \\
7.36 & 7.26 & 9.46
\end{array}\right) \mathrm{eV}
\end{gathered}
$$

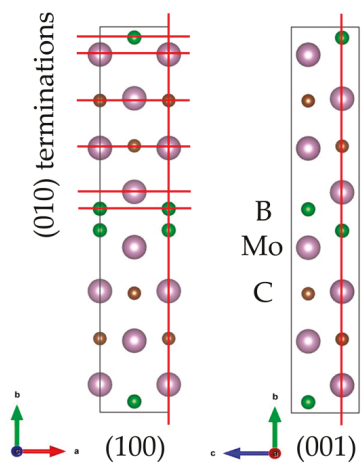

Figure 3. Considered (100), (001) surfaces and (010) surface terminations for the determination of the surface binding potentials in the ab initio approach. The colored spheres represent Mo atoms in purple, $\mathrm{B}$ atoms in green, and $\mathrm{C}$ atoms in brown. The figure was made with VESTA [46].

\subsubsection{SIMTRA}

Within SIMTRA simulations, $1 \times 10^{7}$ particles for Mo and $5 \times 10^{6}$ particles for B and C corresponding to a 2:1:1 target composition were transported. For the simulation setup, a cylinder with a diameter of $0.16 \mathrm{~m}$ and a length of $0.334 \mathrm{~m}$ was used. The target was positioned in the center of the simulation chamber. Seven circular substrates with a radius of $5 \mathrm{~mm}$ were arranged in the chamber corresponding to the actual experimental setup. The gas temperature was set to $300 \mathrm{~K}$. The atomic interaction was described with the Lenz-Jensen screening function implemented in SIMTRA. Gas motion and diffusion is considered within the gas transport. The racetrack profile of the target used for the experimental work was measured by a profilometer and taken into account for the simulations. The simulations were carried out in vacuum $\left(p_{\mathrm{Ar}}=1 \times 10^{-9} \mathrm{~Pa}\right)$ and in $\mathrm{Ar}$ and $\mathrm{Kr}$ gaseous atmosphere at pressures utilized in the experiments (Table 1). Atoms redeposited on the target during deposition are not sputtered again within the simulation. To overcome this virtual loss of particles, atoms redeposited on the target are distributed on all surfaces within the utilized 
simulation chamber with respect to the initial particle distribution, including the influence of the angular distribution function. For this, the ratio of deposited atoms on a substrate divided by the total number of sputtered atoms was multiplied by the number of deposited atoms on the target surface and added to the specific substrate.

\section{Results and Discussion}

\subsection{Experiment}

The angle- and pressure-dependent film compositions for both sputtering gases, Ar and $\mathrm{Kr}$, are presented in Figure 4. The target composition is indicated by black solid lines. For both sputter gases, the angle-dependence of Mo is convex, while the lighter elements B and C show a concave angle-dependence. At $\alpha \leq 22.5^{\circ}$ (Figure 2), a deficiency of the heavy element (Mo) and a surplus of light elements (B and C) is measured. Mo exhibits a deficiency of up to 18 at.\%, while B and $\mathrm{C}$ exhibit a surplus of up to 9 at.\% with respect to the target composition. The opposite trend is observed for $\alpha \geq 45^{\circ}$. Hence, the film composition while sputtering from a $\mathrm{Mo}_{2} \mathrm{BC}$ target is angle-dependent, which was previously observed by Olsen et al. [35] for sputtering (metallic) alloy targets. They explained mass-dependent angular distribution functions by backscattering of light elements on the heavier elements within the collision cascade in the target [35], resulting in an enrichment of lighter elements in directions normal to the target surface. Obviously, Mo cannot be backscattered due to reflective collisions with lighter elements, such as B and C.

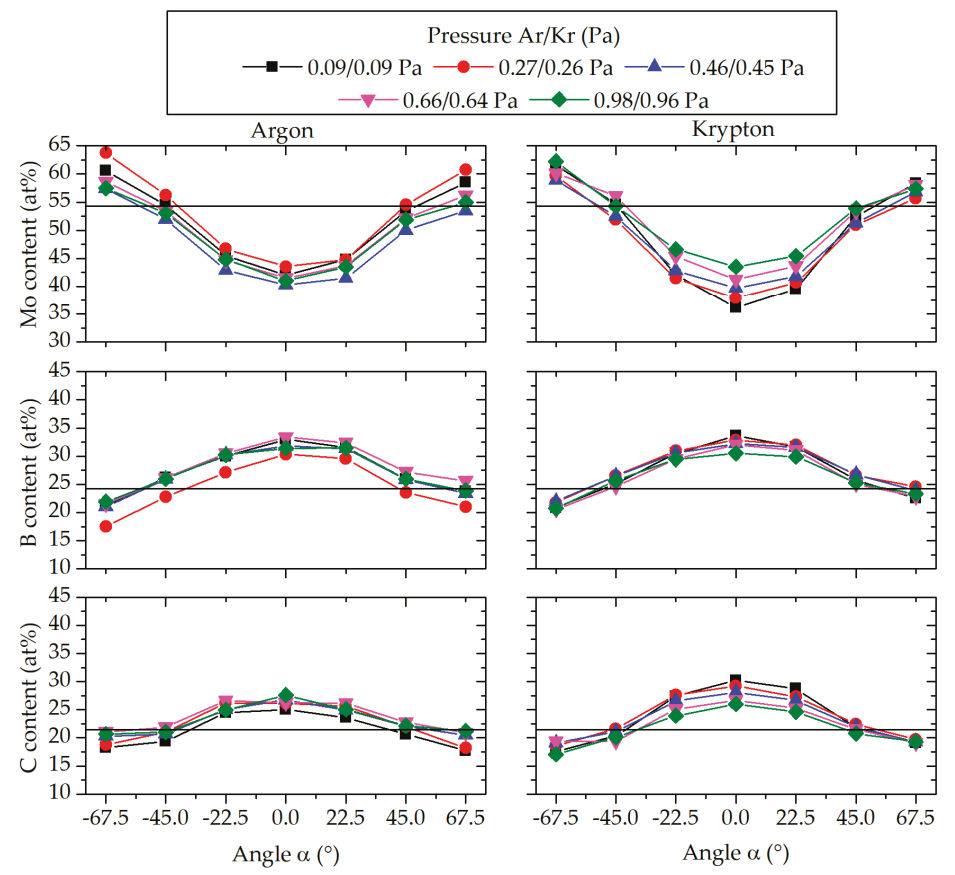

Figure 4. Angle-resolved composition evolution of the deposited thin films within the pressure range from 0.1 to $1.0 \mathrm{~Pa}$. The first pressure value pertains to the Ar depositions, the second value to the $\mathrm{Kr}$ depositions. The average oxygen content was less than 1.5 at.\% for all depositions and not considered further. The target composition is marked by the black horizontal lines. 
Comparing the Mo content of Ar and $\mathrm{Kr}$ depositions, a clear pressure-dependence can be seen for $\mathrm{Kr}$, while no significant composition changes were obtained for Ar. For Kr sputtering at $\alpha=0^{\circ}$, the Mo content changes from 36 at. $\%$ at $0.09 \mathrm{~Pa}$ to 43 at. $\%$ at $0.96 \mathrm{~Pa}$. The chemical variation at $\alpha= \pm 45^{\circ}$ is less distinct, while at $\alpha= \pm 67.5^{\circ}$, the Mo content variations are within the measurement error. For gas phase scattering of $\mathrm{B}$ and $\mathrm{C}$ in $\mathrm{Kr}$, the opposite trend is observed regarding the angle-dependent composition variation. However, the chemical variations due to pressure changes are within the measurement error. It is evident that an increase in pressure leads to a chemical composition closer to the nominal target composition and hence, stoichiometry. In an effort to determine the cause for the here observed sputtering gas-induced composition deviations, simulations were carried out, which allow for an independent analysis of composition deviations caused by sputtering of the target and scattering during the gas phase transport.

\subsection{Simulations}

The angle- and pressure-dependent film compositions with surface binding potentials (SBV's) determined by the energy conservation law and ab initio approaches, as discussed above, are presented in Figure 5 for depositions in Ar.

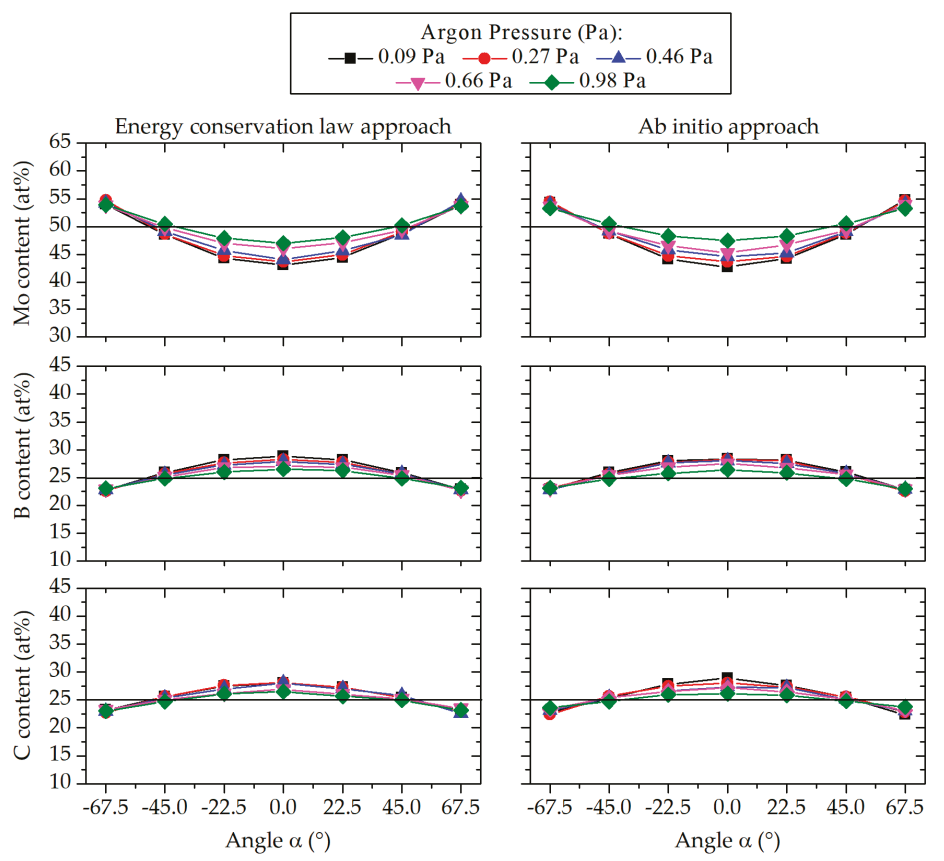

Figure 5. The simulated angle-resolved composition of thin films with the Ar pressure range from 0.09 to $0.98 \mathrm{~Pa}$. Considered surface binding energies of the two approaches (left) energy conservation law and (right) ab initio. The ideal stoichiometric target composition is marked by the black horizontal lines.

The trend of the experimentally determined angle- and pressure-dependent film composition depicted in Figure 4 is reproduced. The angle-dependence of Mo is convex, while B and C show a concave angle-dependence. Films at $\alpha \leq 22.5^{\circ}$ exhibit a deficiency of the heavy Mo and an enrichment of light B and C. As in the experimental data for $\alpha>45^{\circ}$, an opposite trend is observed. The maximum difference in SBVs determined by the energy conservation law and ab initio approaches is $32 \%$. This SBV difference leads to composition differences of less or equal to 0.9 at. $\%$ and 1.1 at.\% for Mo sputtered in Ar (Figure 5) and Kr (not shown), respectively. The magnitude of these composition 
differences cannot be resolved by EDX as the expected experimental errors are larger than the composition differences. For all simulations discussed below, SBVs determined by the ab initio approach were employed.

Pressure changes affect the target voltage and hence the ion energies impinging on the target (see Table 1). The influence of the ion energy on the ADF is illustrated in Figure 6. Within these simulations, scattering events during gas phase transport are deliberately not considered by utilizing an Ar pressure of $10^{-9} \mathrm{~Pa}$. Hence, these simulations only describe sputtering, specifically the effect of the kinetic energy of $\mathrm{Ar}^{+}$and $\mathrm{Kr}^{+}$on the angle-dependent composition of the sputtered flux. These simulations will therefore be referred to as initial ADFs. Increasing the kinetic energy of $\mathrm{Ar}^{+}$from 314 to $401 \mathrm{eV}$ (by $27 \%$ ) results in absolute mean composition differences of less than or equal to 0.4 at. $\%$ for all simulations. Hence, the absolute, ion energy-induced composition changes in the sputtered flux are on average one order of magnitude smaller than the expected measurement error and hence could not be resolved by EDX measurements.

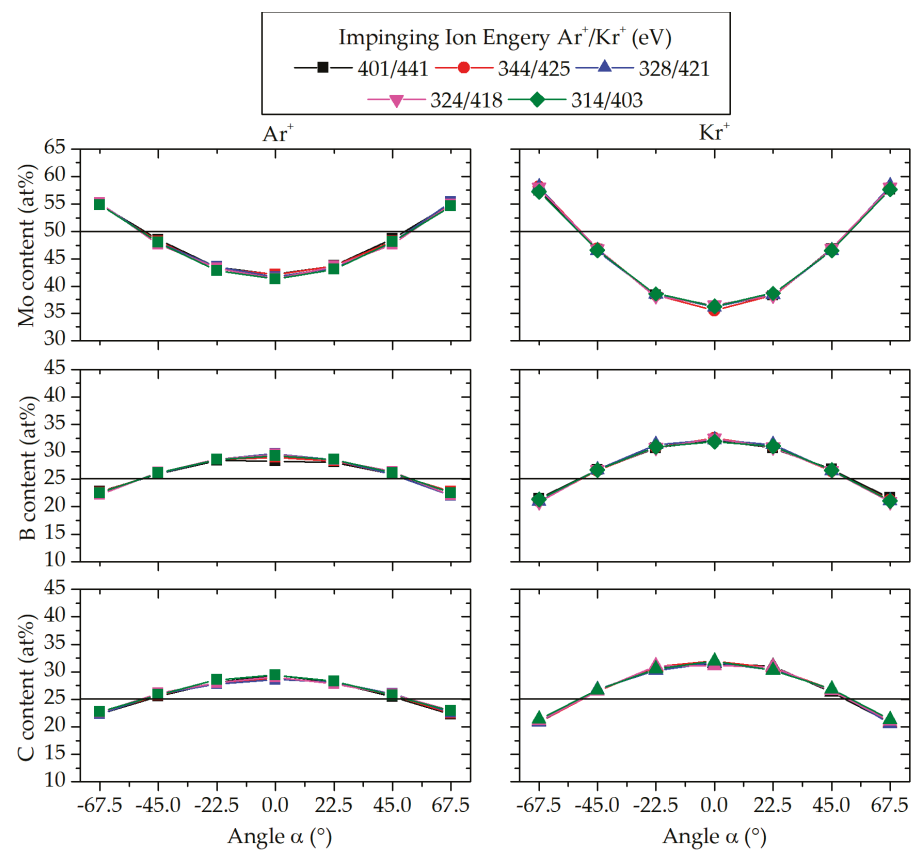

Figure 6. Angle-resolved composition evolution of the sputtered flux for different impinging ion energies of $\mathrm{Ar}^{+}$(left) and $\mathrm{Kr}^{+}$(right) ions. The first energy value pertains to $\mathrm{Ar}^{+}$sputtering, the second value to $\mathrm{Kr}^{+}$sputtering. The ideal stoichiometric target composition is marked by the black horizontal lines.

The initial ADF of Mo sputtered by $\mathrm{Ar}^{+}$(see Figure 6) exhibits a convex distribution, resulting in an Mo deficiency of 8 at. $\%$ at $\alpha=0^{\circ}$ with respect to a nominal Mo content of 50 at. $\%$. At $\alpha= \pm 67.5^{\circ}$, a surplus of 5 at.\% Mo is obtained. Both light elements exhibit a concave distribution, resulting in a surplus of 4 at. $\%$ at $\alpha=0^{\circ}$ and a deficiency of 3 at. $\%$ at $\alpha= \pm 67.5^{\circ}$ with respect to a nominal light element content of 25 at. $\%$ each. Sputtering by $\mathrm{Kr}^{+}$(Figure 6) leads to more pronounced convex and concave distributions for heavy and light elements, respectively. The Mo deficiency and surplus are increased to 14 at. $\%$ and 8 at. $\%$, respectively. For both light elements, a surplus of 7 at. $\%$ and a deficiency of 4 at. $\%$ can be found at $\alpha=0^{\circ}$ and $\pm 67.5^{\circ}$, respectively. Compared to Ar, the sputtering-induced differences of $\mathrm{ADF}$ in $\mathrm{Kr}$ result in larger deviations between the composition of the target and the 
angle-dependent sputtered flux. These results can be rationalized based on the above discussed mass-dependent reflective collisions within the target surface. In the collision cascade, only B and C can be backscattered by Mo, leading to a preferential ejection of $\mathrm{B}$ and $\mathrm{C}$ close to the target normal. Mo cannot be backscattered due to a reflective collision with lighter B or C.

Simulations of the film composition that take, in addition to sputtering at the target, the scattering events within gas phase transport into account, are shown in Figure 7. The Ar or Kr pressures are identical to the experimental pressures depicted in Table 1. Generally, the experimentally-determined angle-dependent film composition data are consistent with the simulation results.

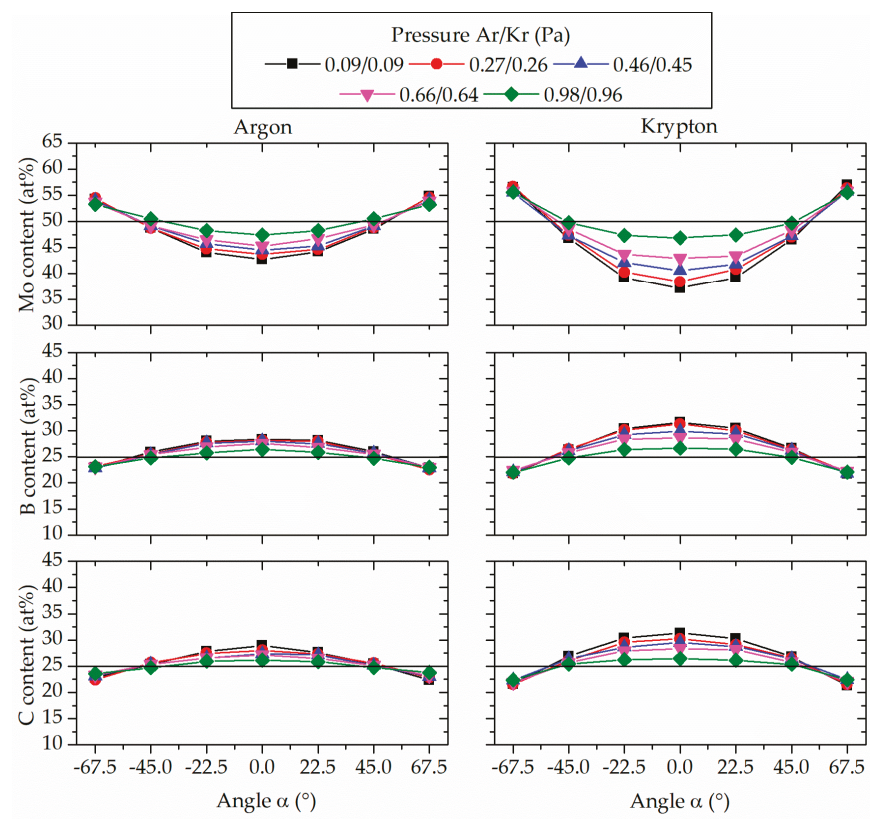

Figure 7. Angle-resolved evolution of simulated film compositions considering sputtering at the target, as well as scattering during gas phase transport. The first pressure value pertains to the Ar depositions, the second value to the $\mathrm{Kr}$ depositions. The ideal stoichiometric target composition is marked by the black horizontal lines.

Significant differences between the initial ADF and the ADF obtained after scattering during transport in the gas phase are obtained for $\mathrm{Ar}$ and $\mathrm{Kr}$ as the pressure is increased by one order of magnitude. An increase in Mo content at $\alpha=0^{\circ}$ of 4.7 at. $\%$ and 9.7 at. $\%$ and for both light elements a decrease of 3 at. $\%$ and 5 at. $\%$ can be obtained in $\mathrm{Ar}$ and $\mathrm{Kr}$, respectively. At $\alpha= \pm 67.5^{\circ}$, no significant pressure-induced impact on the chemical composition can be observed. Generally, the pressure-induced variations in chemical composition are more pronounced in $\mathrm{Kr}$ and are in good agreement with the experimentally-determined data. Comparison to the EDX composition measurement error indicates that the pressure-dependent composition variations simulated in Ar cannot be resolved experimentally.

To identify the cause of the here discussed angle- and pressure-dependent film composition variations, the angle-resolved average trajectory lengths of the sputtered species are calculated. The average trajectory length, $d$, is the mean distance a particle travels from sputtering at the target to deposition at the substrate surface and is maximized for scattering events at large scattering angles and short mean free paths. The pressure-dependence of $d$ is shown in Figure 8 for Ar and Kr. 


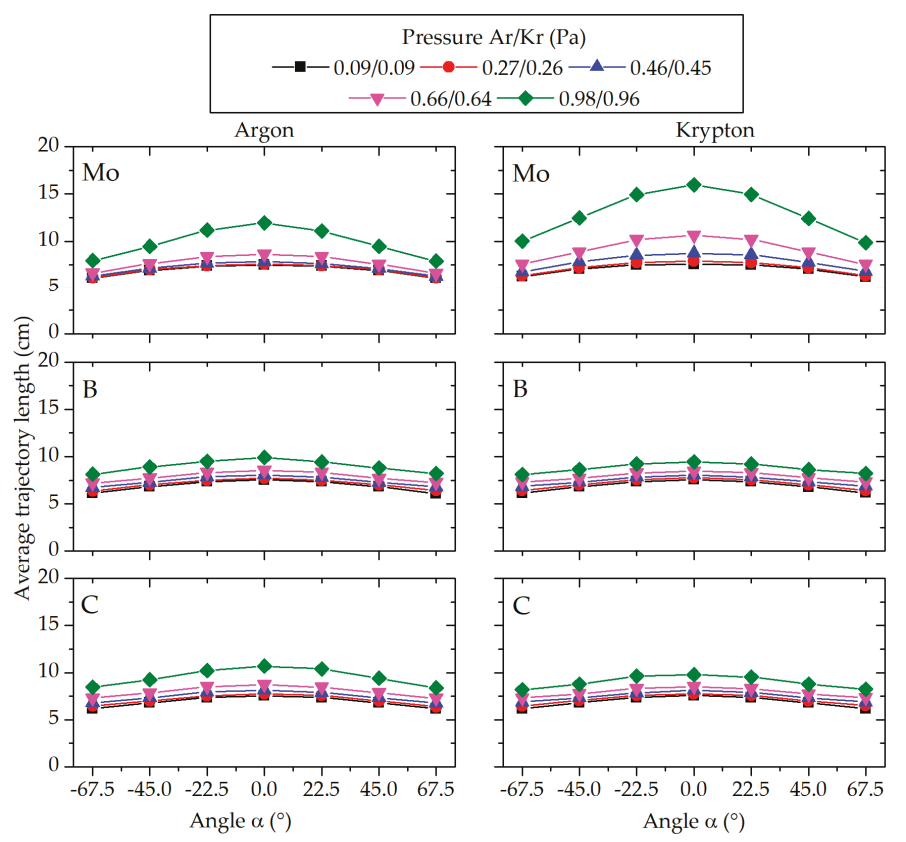

Figure 8. Simulated average trajectory length of the sputtered atoms transported through the gas phase at given $\mathrm{Ar}$ (left) and Krypton (right) pressures. The first pressure value pertains to the Ar depositions, the second value to the $\mathrm{Kr}$ depositions.

Increasing the Ar pressure by one order of magnitude results in a relative increase of $d$ at $\alpha=0^{\circ}$ of $59 \%, 31 \%$, and $42 \%$ for $\mathrm{Mo}, \mathrm{B}$, and $\mathrm{C}$, respectively. The same change in $\mathrm{Kr}$ pressure results in a relative increase of $\mathrm{d}$ at $\alpha=0^{\circ}$ of $111 \%, 25 \%$, and $29 \%$ for Mo, B, and C, respectively. Hence, the average Mo trajectory length is up to $34 \%$ larger in $\mathrm{Kr}$ than in Ar.

The average number of Mo collisions at the maximum $\mathrm{Ar}$ and $\mathrm{Kr}$ pressures at $\alpha=0^{\circ}$ is 19.4 and 22.9 , respectively, exhibiting a relative difference of $18.2 \%$. As the pressure-induced increase in average trajectory length $d$ is caused by the number of collisions, as well as the average scattering angle, simulations were conducted where the number of collisions was kept constant to unravel the contribution of the average scattering angle. For each element, one additional simulation was conducted at a specific $\mathrm{Kr}$ pressure (0.89 Pa for Mo and 1.10 Pa for B and $\mathrm{C}$ ) to match the number of collisions computed for scattering in $0.98 \mathrm{~Pa}$ of $\mathrm{Ar}$, which are 19.4, 10.7, and 12.4, for Mo, $\mathrm{B}$, and $C$, respectively.

At a constant average number of Mo collisions of 19.4, the pressure-induced increase in $d$ of Mo is 23\% larger in $\mathrm{Kr}$ than in Ar. At an average number of collisions of 10.7 and 12.4 for B and C, respectively, a pressure-induced increase in $\mathrm{d}$ of $1.3 \%$ and $0.7 \%$ was obtained for $\mathrm{B}$ and $\mathrm{C}$, respectively. Hence, it is deduced that the average scattering angle of Mo is significantly larger in $\mathrm{Kr}$ than in Ar and that the evolution of the angle- and pressure-dependent film composition is determined by the average scattering angle of Mo. Assuming energy and momentum conservation, a mass-dependent expression for the maximum scattering angle of a particle with a mass larger than the gas species is given by Equation (6) $[47,48]$, where $\vartheta_{\max }$ is the maximum scattering angle and $m_{\mathrm{Mo}}$ and $m_{i}$ are the masses of Mo and the gas atom, respectively. Consequently, maximum scattering angles for Mo of $24.6^{\circ}$ and $60.4^{\circ}$ in $\mathrm{Ar}$ and $\mathrm{Kr}$, respectively, were obtained for the masses of 95.96, 39.95, and $83.80 \mathrm{amu}$ 
for Mo, Ar, and Kr, respectively [49]. Hence, the above deduced larger average scattering angle for Mo in $\mathrm{Kr}$ as compared to Ar is caused by the mass ratio between the sputtering gas and Mo.

$$
\vartheta_{\max }=\arcsin \left(\frac{m_{\text {gas }}}{m_{\mathrm{Mo}}}\right)
$$

The simulations carried out within this work allowed an independent consideration of the sputtering process at the target surface, as well as the scattering events within the gas phase transport. Pressure variations over one order of magnitude insignificantly influence the sputtering process, whereas the mass of the impinging ion exhibits a strong impact on the initial ADF. Sputtering-induced differences between the target and thin film composition caused by $\mathrm{Kr}^{+}$are larger compared to sputtering with $\mathrm{Ar}^{+}$, which is in agreement with the sputtering experiments at low pressures.

Gas phase scattering events induced-variations in film chemical composition depend on both the gas pressure and mass of the gas atom. The average trajectory length was shown to be a good indicator for the impact of scattering. To unravel the relative contribution of numbers of collision and average scatter angle, simulations with an identical number of collisions in $\mathrm{Ar}$ and $\mathrm{Kr}$ of 19.4, 10.7, and 12.4, for Mo, B, and C, respectively, were conducted. In Kr compared to Ar a dominant pressure-induced increase in $d$ of $23 \%$ for Mo, compared to $1.3 \%$ and $0.7 \%$ for $\mathrm{B}$, and C, respectively, was obtained. Hence, the significantly larger average trajectory length of $\mathrm{Mo}$ in $\mathrm{Kr}$ as compared to Ar at the same number of collisions can be rationalized by the larger average scattering angle of Mo, which in turn controls the evolution of the angle- and pressure-dependent film composition.

\section{Conclusions}

The evolution of the angle-resolved composition of Mo-B-C thin films deposited from a $\mathrm{Mo}_{2} \mathrm{BC}$ compound target was investigated experimentally and theoretically as a function of the Ar and $\mathrm{Kr}$ pressure. Samples were positioned in a specific angular arrangement from $\alpha=0^{\circ}$ to $\pm 67.5^{\circ}$ with respect to the target normal with a fixed target substrate distance.

Considering the simulated mass-dependent initial angular distribution functions, a convex distribution for Mo was observed, whereas $B$ and $C$ exhibited concave distributions as a consequence of reflective collisions in the collision cascade. $\mathrm{B}$ and $\mathrm{C}$ can only be backscattered by the heavy Mo leading to the preferential ejection of B and C close to the target normal. Obviously, Mo cannot be backscattered due to a reflective collision with a lighter element.

Within experiments and simulations, the observed change in angle-resolved composition as a result of a by one order of magnitude increased Ar pressure was lower than the expected measurement error and hence, cannot be resolved by EDX. On the contrary, sputtering by $\mathrm{Kr}^{+}$results in significantly larger deviations between the target and the film composition. These deviations can be rationalized based on reflective collisions in the collision cascade. As the $\mathrm{Kr}$ pressure is increased, scattering during transport in the gas phase results in angular resolved compositions that approach the target composition. Furthermore, based on considering the relative contributions of the number of collisions and scatter angle to the average trajectory length, it is inferred that the significantly larger average trajectory length of Mo in $\mathrm{Kr}$ compared to Ar can be rationalized by an on average larger scattering angle of Mo. It is shown that the mass ratio between sputtering gas and sputtered species defines the scattering angle within the collision cascades in the target, as well as for the collisions in the gas phase, which in turn define the angle- and pressure-dependent film compositions.

Author Contributions: Conceptualization, J.-O.A., S.M. and J.M.S.; Methodology, J.-O.A., S.M. and D.P.; Investigation, J.-O.A., S.M. and D.P.; Writing, J.-O.A., S.M., D.P. and J.M.S.; Project administration, J.-O.A., S.M., J.M.S. and J.-O.A.; Funding acquisition, J.M.S.

Funding: The authors gratefully acknowledge the financial support granted by the DFG SCHN 735/35-1. 
Acknowledgments: The authors gratefully acknowledge G. Dehm for fruitful discussions. The simulations were performed with computing resources granted by JARAHPC from RWTH Aachen University under Project No. JARA0131. Support by VR-RFI (contracts \#821-2012-5144 \& \#2017-00646_9) and the Swedish Foundation for Strategic Research (SSF, contract RIF14-0053) supporting accelerator operation at Uppsala University is gratefully acknowledged.

Conflicts of Interest: The authors declare no conflict of interest.

\section{References}

1. Bovin, J.O.; O'Keeffe, M.; Stenberg, L. Planar defects in $\mathrm{Mo}_{2} \mathrm{BC}$. An electron microscope study. J. Solid State Chem. 1977, 22, 221-231. [CrossRef]

2. Jeitschko, W.; Nowotny, H.; Benesovsky, F. Die kristallstruktur von $\mathrm{Mo}_{2} \mathrm{BC}$. Monatshefte für Chemie und verwandte Teile anderer Wissenschaften 1963, 94, 565-568. [CrossRef]

3. Emmerlich, J.; Music, D.; Braun, M.; Fayek, P.; Munnik, F.; Schneider, J.M. A proposal for an unusually stiff and moderately ductile hard coating material: $\mathrm{Mo}_{2}$ BC. J. Phys. D Appl. Phys. 2009, 42, 185406. [CrossRef]

4. Bolvardi, H.; Emmerlich, J.; Music, D.; von Appen, J.; Dronskowski, R.; Schneider, J.M. Systematic study on the electronic structure and mechanical properties of $X_{2} \mathrm{BC}(X=\mathrm{Mo}, \mathrm{Ti}, \mathrm{V}, \mathrm{Zr}, \mathrm{Nb}, \mathrm{Hf}, \mathrm{Ta}$ and $\mathrm{W})$. J. Phys. Condens. Matter 2012, 25, 045501. [CrossRef]

5. Bolvardi, H.; Emmerlich, J.; Mráz, S.; Arndt, M.; Rudigier, H.; Schneider, J.M. Low temperature synthesis of $\mathrm{Mo}_{2} \mathrm{BC}$ thin films. Thin Solid Films 2013, 542, 5-7. [CrossRef]

6. Sarakinos, K.; Alami, J.; Konstantinidis, S. High power pulsed magnetron sputtering: A review on scientific and engineering state of the art. Surf. Coat. Technol. 2010, 204, 1661-1684. [CrossRef]

7. Ohring, M. Materials Science of Thin Films, 2nd ed.; Academic Press Limited: Cambridge, MA, USA, 1992.

8. Emmerlich, J.; Högberg, H.; Sasvári, S.; Persson, P.O.; Hultman, L.; Palmquist, J.P.; Jansson, U.; Molina-Aldareguia, J.M.; Czigány, Z. Growth of $\mathrm{Ti}_{3} \mathrm{SiC}_{2}$ thin films by elemental target magnetron sputtering. J. Appl. Phys. 2004, 96, 4817-4826. [CrossRef]

9. Eklund, P.; Joelsson, T.; Ljungcrantz, H.; Wilhelmsson, O.; Czigány, Z.; Högberg, H.; Hultman, L. Microstructure and electrical properties of Ti-Si-C-Ag nanocomposite thin films. Surf. Coat. Technol. 2007, 201, 6465-6469. [CrossRef]

10. Chen, L.; Holec, D.; Du, Y.; Mayrhofer, P.H. Influence of $\mathrm{Zr}$ on structure, mechanical and thermal properties of Ti-Al-N. Thin Solid Films 2011, 519, 5503-5510. [CrossRef] [PubMed]

11. Eklund, P.; Beckers, M.; Jansson, U.; Högberg, H.; Hultman, L. The $\mathrm{M}_{n+1} \mathrm{AX}$ 住 phases: Materials science and thin-film processing. Thin Solid Films 2010, 518, 1851-1878. [CrossRef]

12. Neidhardt, J.; Mráz, S.; Schneider, J.M.; Strub, E.; Bohne, W.; Liedke, B.; Moller, W.; Mitter, C. Experiment and simulation of the compositional evolution of Ti-B thin films deposited by sputtering of a compound target. J. Appl. Phys. 2008, 104, 063304. [CrossRef]

13. Mitterer, C. Borides in thin film technology. J. Solid State Chem. 1997, 133, 279-291. [CrossRef]

14. Kunc, F.; Musil, J.; Mayrhofer, P.H.; Mitterer, C. Low-stress superhard Ti-B films prepared by magnetron sputtering. Surf. Coat. Technol. 2003, 174, 744-753. [CrossRef]

15. Shaginyan, L.R.; Mišina, M.; Kadlec, S.; Jastrabik, L.; Mackova, A.; Peřina, V. Mechanism of the film composition formation during magnetron sputtering of WTi. J. Vac. Sci. Technol. A 2001, 19, 2554-2566. [CrossRef]

16. Jonsson, L.B.; Hedlund, C.; Katardjiev, I.V.; Berg, S. Compositional variations of sputter deposited Ti/W barrier layers on substrates with pronounced surface topography. Thin Solid Films 1999, 348, 227-232. [CrossRef]

17. Ramarotafika, H.; Lemperiere, G. Influence of a d.c. substrate bias on the resistivity, composition, crystallite size and microstrain of WTi and WTi-N films. Thin Solid Films 1995, 266, 267-273. [CrossRef]

18. Rogers, B.R.; Tracy, C.J.; Cale, T.S. Compositional variation in sputtered Ti-W films due to re-emission. J. Vac. Sci. Technol. A 1994, 12, 2980-2984. [CrossRef]

19. Rogers, B.R.; Cale, T.S.; Chang, Y.K. Simulation and experimental study of re-emission during sputter deposition of Ti-W films. J. Vac. Sci. Technol. A 1996, 14, 1142-1146. [CrossRef]

20. Bergstrom, D.B.; Tian, F.; Petrov, I.; Moser, J.; Greene, J.E. Origin of compositional variations in sputter-deposited $\operatorname{Ti}_{x} \mathrm{~W}_{1-x}$ diffusion barrier layers. Appl. Phys. Lett. 1995, 67, 3102-3104. [CrossRef] 
21. Rossnagel, S.M.; Yang, I.; Cuomo, J.J. Compositional changes during magnetron sputtering of alloys. Thin Solid Films 1991, 199, 59-69. [CrossRef]

22. Dirks, A.G.; Wolters, R.A.M.; Nellissen, A.J.M. On the microstructure-property relationship of W-Ti-(N) diffusion barriers. Thin Solid Films 1990, 193, 201-210. [CrossRef]

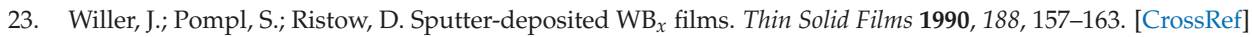

24. Simao, R.A.; Costa, A.K.; Achete, C.A.; Camargo Jr, S.S. Magnetron sputtering SiC films investigated by AFM. Thin Solid Films 2000, 377, 490-494. [CrossRef]

25. Murakami, Y.; Shingyoji, T. Compositional difference between films and targets in sputtering of refractory metal silicides. J. Vac. Sci. Technol. A 1990, 8, 851-854. [CrossRef]

26. Yamazaki, T.; Ikeda, N.; Tawara, H.; Sato, M. Investigation of composition uniformity of MoSi $i_{x}$ sputtering films based on measurement of angular-distribution of sputtered atoms. Thin Solid Films 1993, 235, 71-75. [CrossRef]

27. Liao, M.Y.; Gotoh, Y.; Tsuji, H.; Ishikawa, J. Deposition of vanadium carbide thin films using compound target sputtering and their field emission. J. Vac. Sci. Technol. A 2005, 23, 1379-1383. [CrossRef]

28. Liao, M.Y.; Gotoh, Y.; Tsuji, H.; Ishikawa, J. Compound-target sputtering for niobium carbide thin-film deposition. J. Vac. Sci. Technol. B 2004, 22, L24-L27. [CrossRef]

29. Mráz, S.; Emmerlich, J.; Weyand, F.; Schneider, J.M. Angle-resolved evolution of the composition of Cr-Al-C thin films deposited by sputtering of a compound target. J. Phys. D Appl. Phys. 2013, 46, 135501. [CrossRef]

30. Rueß, H.; to Baben, M.; Mráz, S.; Shang, L.; Polcik, P.; Kolozsvári, S.; Hans, M.; Primetzhofer, D.; Schneider, J.M. HPPMS deposition from composite targets: Effect of two orders of magnitude target power density changes on the composition of sputtered Cr-Al-C thin films. Vacuum 2017, 145, 285-289. [CrossRef]

31. Walter, C.; Martinez, C.; El-Raghy, T.; Schneider, J.M. Towards large area MAX phase coatings on steel. Steel Res. Int. 2005, 76, 225-228. [CrossRef]

32. Palmquist, J.P.; Jansson, U.; Seppänen, T.; Persson, P.; Birch, J.; Hultman, L.; Isberg, P. Magnetron sputtered epitaxial single-phase $\mathrm{Ti}_{3} \mathrm{SiC}_{2}$ thin films. Appl. Phys. Lett. 2002, 81, 835-837. [CrossRef]

33. Eklund, P.; Beckers, M.; Frodelius, J.; Högberg, H.; Hultman, L. Magnetron sputtering of $\mathrm{Ti}_{3} \mathrm{SiC}_{2}$ thin films from a compound target. J. Vac. Sci. Technol. A 2007, 25, 1381-1388. [CrossRef]

34. Jo, Y.H.; Mohanty, B.C.; Yeon, D.H.; Lee, S.M.; Cho, Y.S. Single elementary target-sputtered $\mathrm{Cu}_{2} \mathrm{ZnSnSe}_{4}$ thin film solar cells. Sol. Energy Mater. Sol. Cells 2015, 132, 136-141. [CrossRef]

35. Olson, R.R.; King, M.E.; Wehner, G.K. Mass effects on angular distribution of sputtered atoms. J. Appl. Phys. 1979, 50, 3677-3683. [CrossRef]

36. Van Aeken, K.; Mahieu, S.; Depla, D. The metal flux from a rotating cylindrical magnetron: A Monte Carlo simulation. J. Phys. D Appl. Phys. 2008, 41, 205307. [CrossRef]

37. Möller, W.; Eckstein, W.; Biersack, J.P. Tridyn-binary collision simulation of atomic collisions and dynamic composition changes in solids. Comput. Phys. Commun. 1988, 51, 355-368. [CrossRef]

38. Möller, W.; Posselt, M. TRIDYN_FZR user manual. Qucosa 2001.

39. Chase, M.W., Jr.; Davies, C.A.; Downey, J.R.; Frurip, D.J.; McDonald, R.A.; Syverud, A.N. JANAF thermochemical tables, 3rd ed. J. Phys. Chem. Ref. Data 1985, 14 (Suppl. 1).

40. Kresse, G.; Hafner, J. Ab initio molecular dynamics for open-shell transition metals. Phys. Rev. B 1993, 48, 13115. [CrossRef]

41. Kresse, G.; Hafner, J. Ab initio molecular-dynamics simulation of the liquid-metal-amorphoussemiconductor transition in germanium. Phys. Rev. B 1994, 49, 14251. [CrossRef]

42. Perdew, J.P.; Burke, K.; Ernzerhof, M. Generalized gradient approximation made simple. Phys. Rev. Lett. 1996, 77, 3865. [CrossRef]

43. Kresse, G.; Joubert, D. From ultrasoft pseudopotentials to the projector augmented-wave method. Phys. Rev. B 1999, 59, 1758. [CrossRef]

44. Blöchl, P.E. Projector augmented-wave method. Phys. Rev. B 1994, 50, 17953. [CrossRef]

45. Monkhorst, H.J.; Pack, J.D. Special points for Brillouin-zone integrations. Phys. Rev. B 1976, $13,5188$. [CrossRef]

46. Momma, K.; Izumi, F. VESTA 3 for three-dimensional visualization of crystal, volumetric and morphology data. J. Appl. Crystallogr. 2011, 44, 1272-1276. [CrossRef] 
47. McDaniel, E.W. Collision Phenomena in Ionized Gases; Wiley: New York, NY, USA, 1964.

48. Behrisch, R. Sputtering by Particle Bombardment I; Springer: Berlin, Germany, 1981.

49. Wieser, M.E.; Berglund, M. Atomic weights of the elements 2007 (IUPAC technical report). Pure Appl. Chem. 2009, 81, 2131-2156. [CrossRef]

(C) 2019 by the authors. Licensee MDPI, Basel, Switzerland. This article is an open access article distributed under the terms and conditions of the Creative Commons Attribution (CC BY) license (http://creativecommons.org/licenses/by/4.0/). 
Article

\title{
The Seebeck Coefficient of Sputter Deposited Metallic Thin Films: The Role of Process Conditions
}

\author{
Florian G. Cougnon and Diederik Depla * \\ Department of Solid State Sciences, Ghent University, Krijgslaan 281 (S1), 9000 Gent, Belgium; \\ florian.cougnon@ugent.be \\ * Correspondence: Diederik.Depla@ugent.be
}

Received: 9 April 2019; Accepted: 26 April 2019; Published: 1 May 2019

\begin{abstract}
Because of their reduced dimensions and mass, thin film thermocouples are a promising candidate for embedded sensors in composite materials, especially for application in lightweight and smart structures. The sensitivity of the thin film thermocouple depends however on the process conditions during deposition. In this work, the influence of the discharge current and residual gas impurities on the Seebeck coefficient is experimentally investigated for sputter deposited copper and constantan thin films. The influence of the layer thickness on the film Seebeck coefficient is also discussed. Our observations indicate that both a decreasing discharge current or an increasing background pressure results in a growing deviation of the film Seebeck coefficient compared to its bulk value. Variations in discharge current or background pressure are linked as they both induce a variation in the ratio between the impurity flux to metal flux towards the growing film. This latter parameter is considered a quantitative measure for the background residual gas incorporation in the film and is known to act as a grain refiner. The observed results emphasize the importance of the domain size on the Seebeck coefficient of metallic thin films.
\end{abstract}

Keywords: Seebeck coefficient; background pressure; impurities; discharge current; domain size; layer thickness; sputter deposition

\section{Introduction}

Thin film sensing applications, such as thin film thermocouples, can be very attractive for lightweight structures, small devices or applications in need of a high temporal, or spatial resolution as they have a very low mass, reduced dimensions, and a very fast response time [1-5]. For example, thin film thermocouples are used for nanoscale thermometry [6-8], for monitoring local temperature distributions on integrated-circuits [9,10], in solid oxide fuel cells [11], and turbine engines [12], or for monitoring sudden temperature changes in cutting tools for machining explosive materials [13]. Furthermore, thin film sensors can be embedded inside composite materials without affecting the structural integrity of the material by their dimensional extent, enabling local and in situ sensing without compromise. In the case of sensing applications, typically the circuit does not draw current but an open-gate voltage is measured and linked to a physical property. Studying the behavior of the Seebeck coefficient of thin films and the relation to the deposition process is therefore an interesting field. The Seebeck effect describes the observation of an induced voltage difference when a temperature difference is applied over a metal or semiconductor. The generated voltage scales linearly to the applied temperature difference and the proportionality factor is referred to as the Seebeck coefficient $S$.

Thermoelectric energy is transported by mobile charge carriers [14]. Typically, metals have a high charge carrier concentration and thus a low Seebeck coefficient but a high electrical conductivity, whereas semiconductors have a low charge carrier concentration and thus a high Seebeck coefficient and low electrical conductivity. For thermoelectric applications, i.e., the conversion of heat into 
electrical power, it is important to combine of a low thermal conductivity (increasing temperature difference), a high Seebeck coefficient (increasing thermoelectric voltage), and a high electrical conductivity (decreasing Ohmic losses). The interplay between these three material properties is summarized under a single parameter $Z=S \sigma^{2} / \kappa$, called the figure-of-merit. Presently, around half of the thermoelectric research and development is focused on the maximization of the figure of merit $\mathrm{Z}$ for semiconductor materials such as $\mathrm{PbTe}, \mathrm{Bi}_{2} \mathrm{Te}_{3}$ and $\mathrm{SiGe}$ [15]. Although semiconductors are expected to have higher Seebeck coefficients than metals, these materials are more expensive, have a more complex charge carrier transport mechanism and can quite often only be RF-sputter deposited which hinders to upscale the obtained results to industrial applications. Therefore, we solely study the behavior of the Seebeck coefficient for DC sputtered metallic thin films from a fundamental point of view. Despite the influence of layer thickness on the Seebeck coefficient of metallic films was already studied in the past [16-23], there is still much to be explored. In this study, we investigate the role of the process conditions, i.e., the discharge current and the background pressure, on the Seebeck coefficient of thin metallic films.

\section{Materials and Methods}

The samples discussed in the scope of this work are deposited in a cuboid stainless steel vacuum chamber with a volume of $0.7 \times 0.62 \times 0.56 \mathrm{~m}^{3}$. The films are grown on acetone-cleaned glass substrates (VWR International, Radnor, PA, USA) for the Seebeck measurements or either on RCA-cleaned silicon wafers in the case of film characterization. The samples were measured by X-ray diffraction (Bruker D8, Billerica, MA, USA) in a Bragg-Brentano configuration with a parallel beam bundle defined by the PolyCap. The peaks in the diffractogram were fitted to a Lorentzian-shaped curve and the Debye-Scherrer equation was used to determine the domain sizes. During all depositions, the pumping speed was kept constant $(\approx 75 \mathrm{l} / \mathrm{s})$ and the substrate was grounded and not externally heated or cooled. The used copper and constantan sputter targets are two inch circular planar targets (purity 99.99\%, Testbourne, Hampshire, UK). The films deposited for Seebeck measurements are patterned using a sputter mask specified in Figure 1. After deposition, a complementary bulk wire is attached to the thin film in order to form a conventional E-type, i.e., $\mathrm{Cu}_{55} \mathrm{Ni}_{45}(\mathrm{film})+$ $\mathrm{Ni}_{90} \mathrm{Cr}_{10}$ (wire), or T-type thermocouple, i.e., $\mathrm{Cu}$ (film) $+\mathrm{Cu}_{55} \mathrm{Ni}_{45}$ (wire). The $\mathrm{Cu}_{55} \mathrm{Ni}_{45}$ and $\mathrm{Ni}_{90} \mathrm{Cr}_{10}$ wire (Goodfellow, Cambridgeshire, UK) had a diameter of $0.125 \mathrm{~mm}$ and a polyimide insulation. The Seebeck measurements are performed with a home-built system. The setup consists of a heating element (MeiVac substrate heater, San Jose, CA, USA) and a water-cooled copper block, separated over a distance of $\approx 8 \mathrm{~cm}$. The bimetallic film-wire junction of the sample is centered on the heating element, whereas the electrodes are centered on the copper block. Thermal paste (Dow Corning, Midland, MI, USA) was applied at both ends of the glass substrate in order to enhance the heating or cooling transfer. The water-cooled copper block is kept at a constant temperature of $11^{\circ} \mathrm{C}$. The heating element is PID-controlled and ramps from room temperature to $60^{\circ} \mathrm{C}$ at a rate of $1{ }^{\circ} \mathrm{C} / \mathrm{min}$. Due to the imposed temperature difference, an open-circuit thermoelectric voltage arises at the electrodes of the film-wire thermocouple. The output of the thermocouples was logged by means of a TC01 (National Instruments, Austin, TX, USA), a thermocouple measurement device with built-in software for data acquisition. In order to provide a good electrical contact with the film for the read-out, a fine copper brush was clamped onto the thin film. The hot junction and cold junction temperature were measured by means of a K-type thermocouple. A Labview code managed all the data and plotted the measured voltage as function of the temperature difference between the hot and cold junction. A straight line was fitted to the data ( $\approx 200$ measurement points) and the slope of this line was used to determine the Seebeck coefficient. The experimental values for the E- and T-type bulk thermocouples (i.e., wire + wire) are used as reference and are $(67.3 \pm 1.4) \mu \mathrm{V} /{ }^{\circ} \mathrm{C}$ and $(46.3 \pm 0.8) \mu \mathrm{V} /{ }^{\circ} \mathrm{C}$ respectively. 


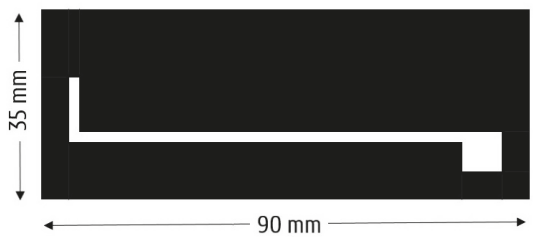

Figure 1. Design of the sputter mask used for pattering the thermocouple leg. The mask is fabricated out of stainless steel and has a thickness of $0.5 \mathrm{~mm}$.

The influence of contamination on the thin film Seebeck coefficient was investigated by progressively increasing the background pressure before deposition. This was achieved by leaking atmospheric air into the vacuum chamber with a mass flow controller (MKS, Andover, MA, USA). After the background pressure is stabilized at a desired value, the sputter gas pressure is set by the introduction of argon gas (Argon 5.0 Praxair, Danbury, CT, USA) in the system. Finally the magnetron discharge is ignited and the target is sputtered at constant current $(0.6 \mathrm{~A})$. The power towards the magnetron is delivered by a Hüttinger 1500DC power supply (Ditzingen, Germany). The target-to-substrate distance and the argon pressure were kept constant during all depositions at $10 \mathrm{~cm}$ and 0.6 Pa. Except otherwise specified, all films, irrespective of the material, had a thickness of $350 \pm 30 \mathrm{~nm}$. The impurity-to-metal impingement flux ratio $\tau$ is used as a measure for the degree of contamination. This parameter is defined as the ratio between the impurity flux and the material flux impinging on the substrate surface, i.e., $\tau=F_{i} / F_{m}$. The material flux was calculated from the measured thickness (Taylor-Hobson profilometer, Leicester, UK), the deposition time, and the film density as measured by $x$-ray reflectometry (Bruker D8). The impurity flux was calculated based on the Maxwell-Boltzmann distribution as presented in previous work [24].

The depositions for the experiment on the influence of the discharge current were performed under the same process conditions as the samples discussed above, i.e., target-to-substrate distance $10 \mathrm{~cm}$ and argon pressure $0.6 \mathrm{~Pa}$. The experiment was performed at two different background pressures. One series was deposited at a low background pressure $\left(3.50 \times 10^{-4} \mathrm{~Pa}\right)$ and one series at a high background pressure $\left(7.50 \times 10^{-3} \mathrm{~Pa}\right)$. The magnetron was sputtered in current-controlled way and the discharge current was varied from 0.6 to $0.1 \mathrm{~A}$. This was done in a random way in order to exclude any effects related to target erosion and/or chamber heating.

\section{Results}

\subsection{Impurities}

Figure 2 presents the results for the deviation of the thin film Seebeck coefficient from the bulk value, i.e., $|\Delta S|=\left|S_{B}-S_{F}\right|$, plotted as function of the impurity-to-metal impingement flux ratio $\tau$ for both copper (a) and constantan (b). In the low-impurity regime, i.e., $\tau \ll 1$, the Seebeck coefficient is not significantly affected by an increased background pressure. The average deviation from the bulk value in this low-impurity regime is represented by the dotted line for both copper $\left(1.9 \mu \mathrm{V} /{ }^{\circ} \mathrm{C}\right)$ and constantan $\left(2.61 \mu \mathrm{V} /{ }^{\circ} \mathrm{C}\right)$. In the high-impurity regime, i.e., $\tau \gg 1$, the Seebeck coefficient is strongly affected by an increasing degree of contamination. For both figures, error bars are included on a single marker but are valid for all data points in the respective plots. In order to simplify the rationale in Section 4.3 , the striped lines in the figures represent the relation $|\Delta S| \sim \tau^{1 / 2}$ where the proportionality factor is fitted to coincide with the data in this high-impurity regime. This specific description of $|\Delta S|$ as function of the impurity-to-metal ratio $\tau$ embodies the thought of a relation between $|\Delta S|$ and the domain size $D$, more specifically $1 / D$. The idea for this relation originates from a previous work [24], where we elaborated a model that accounts for the experimental observation $D \sim \tau^{-1 / 2}$ in the high-impurity regime. The agreement between the experiment and the proposed relation $|\Delta S| \sim \tau^{1 / 2}$ 
is evaluated by means of a chi-squared test. For copper and constantan, we find a value $\chi^{2}=1.98$ (12 data points) and $\chi^{2}=3.21$ (9 data points) respectively. In both cases, the significance of the description is guaranteed within the $95 \%$ confidence interval. The full gray lines in Figure 2 represent the maximal deviation $|\Delta S|_{\max }$ which is found when $S_{F} \rightarrow 0$ and thus $|\Delta S|_{\max }=\left|S_{B}\right|$. We find for copper and constantan a value of $6.2 \mu \mathrm{V} /{ }^{\circ} \mathrm{C}$ and $40.1 \mu \mathrm{V} /{ }^{\circ} \mathrm{C}$ [25] respectively. Copper has a much smaller Seebeck coefficient in comparison to constantan. This hinders the electronic measurement and results in a high signal-to-noise ratio. As the observed trends are similar for both materials, we focus our research on constantan as the latter material has a higher Seebeck coefficient.

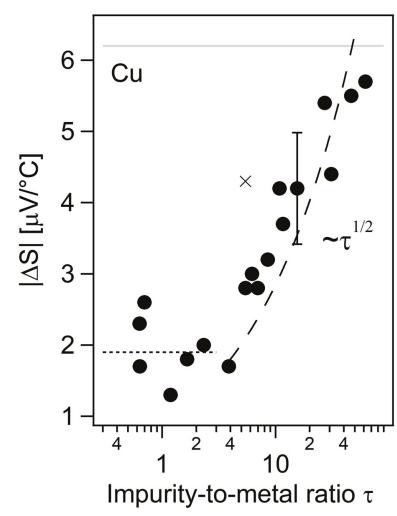

(a)

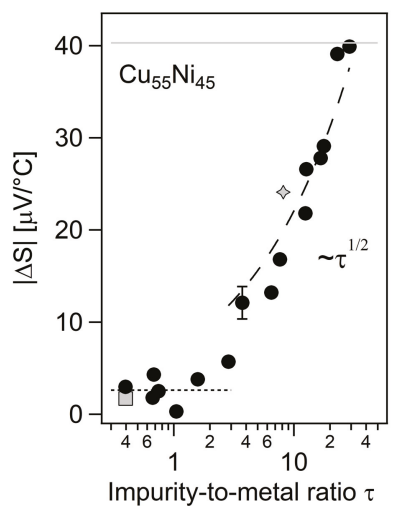

(b)

Figure 2. The deviation of the thin film Seebeck coefficient from the bulk value plotted as function of the impurity-to-metal impingement flux ratio for (a) copper and (b) constantan. The depositions are performed at a discharge current of $0.6 \mathrm{~A}$, an argon pressure of $0.6 \mathrm{~Pa}$ and a target-to-substrate distance of $10 \mathrm{~cm}$. The impurity-to-metal ratio was increased by leaking atmospheric impurities inside the vacuum chamber. The full gray lines in the figures indicate the maximal deviation $|\Delta S|_{\max }$ for copper $\left(6.2 \mu \mathrm{V} /{ }^{\circ} \mathrm{C}\right)$ and constantan $\left(40.1 \mu \mathrm{V} /{ }^{\circ} \mathrm{C}\right)$. The dotted lines represent the average deviation from the bulk value in the low-impurity regime for copper $\left(1.9 \mu \mathrm{V} /{ }^{\circ} \mathrm{C}\right)$ and constantan $\left(2.61 \mu \mathrm{V} /{ }^{\circ} \mathrm{C}\right)$ and the striped lines represent the power law $|\Delta S| \sim \tau^{1 / 2}$. Error bars are included on a single marker but are valid for all data points in the respective plots. The data point represented by the cross marker (a) was excluded from the fit for the proportionality factor. The gray square and diamond markers (b) represent data points from the experiment discussed in Section 3.2.

\subsection{Discharge Current}

The results for the influence of the discharge current on the thin film Seebeck coefficient are presented in Figure 3. The square markers and diamond markers represent depositions performed at a background pressure of $3.50 \times 10^{-4} \mathrm{~Pa}$ and $7.50 \times 10^{-3} \mathrm{~Pa}$ respectively. The values for $|\Delta S|$ are significantly larger for the series deposited at a high background pressure compared to the series deposited at a low background pressure, an observation in accordance to the results discussed in Figure 2. As an illustration, the data points obtained in this experiment which corresponds to the same deposition conditions as the previous experiment, i.e., a discharge current of $0.6 \mathrm{~A}$, are represented by the gray (square and diamond) markers on the plot in Figure 2. For both series, the measurements indicate an increasing deviation of the film Seebeck coefficient from the bulk value with decreasing discharge current. 


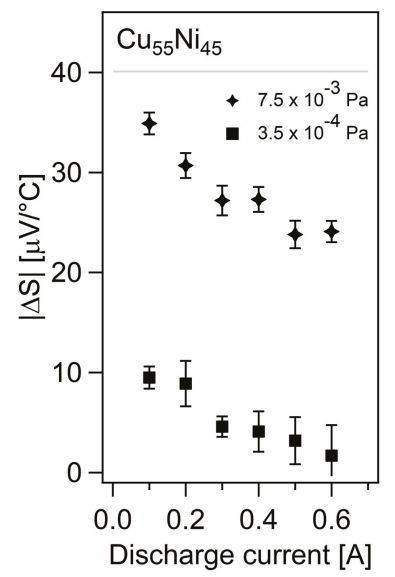

Figure 3. The deviation of the thin film Seebeck coefficient from the bulk value measured as function of the discharge current for 2 different background pressures: $3.50 \times 10^{-4} \mathrm{~Pa}$ (square markers) and $7.50 \times 10^{-3} \mathrm{~Pa}$ (diamond markers). The argon pressure was set to $0.6 \mathrm{~Pa}$ and the target-to-substrate distance was $10 \mathrm{~cm}$. The full gray line indicates the maximal deviation $|\Delta S|_{\max }$.

\subsection{Domain Size}

The domain sizes corresponding to the series deposited at varying discharge current at both low and high background pressure are plotted in Figure 4a. The domain size decreases with increasing background pressure and decreasing discharge current. As the discharge current is varied from $0.6 \mathrm{~A}$ up to $0.1 \mathrm{~A}$, the deposition speed was varied from $1.28 \mathrm{~nm} / \mathrm{s}$ up to $0.23 \mathrm{~nm} / \mathrm{s}$ respectively. Given the constant background pressure used within each series, the ratio of the impurity-to-metal impingement flux ratio $\tau$ thus increases with decreasing discharge current. The variation in background pressure between both series did not have any influence on the deposition speed. Figure $4 \mathrm{~b}$ is based on the same data for the domain size as in Figure 4a but presented in a normalized way and as function of the impurity-to-metal impingement flux ratio $\tau$. The data for the domain size have been normalized in order to allow a better comparison with the additional data set (gray circles) included in Figure $4 \mathrm{~b}$ which originates from a previous experiment [24]. The uppermost dotted line in the figures indicate the averaged domain size in this low-impurity regime. The domain size in the high-impurity regime $(\tau \gg 1)$ is strongly refined by the presence of the impurities during growth. Based on previous research [24], we expect a relation $D \sim \tau^{-1 / 2}$ in this regime. This relation is represented by the striped line and further consolidated by the gray markers taken from the previous work. In the low-impurity regime $(\tau \ll 1)$, the domain sizes are larger and less affected by an increase in impurity-to-metal impingement flux ratio. It must be noted here that in the determination of the domain sizes from the XRD data, we did not account for microstrain contributions. However, this analysis was carried out on the additional data set (gray circles Figure 4) from previous work, but no systematic variation in microstrain was observed.

Figure 5 summarizes the effect of background pressure (square markers vs. diamond markers) and discharge current (square markers and diamond markers) on $|\Delta S|$ described as function of the inverse of the domain size, i.e., $1 / D$. Additional data from the work of Barber et al. [26] are also included on the figure (round gray markers and unfilled blue markers). Whereas the data in the latter work are presented as $|\Delta S|$ as function of the FWHM, they are recalculated here using the Scherrer-equation in order to present them as function of $1 / D$. 


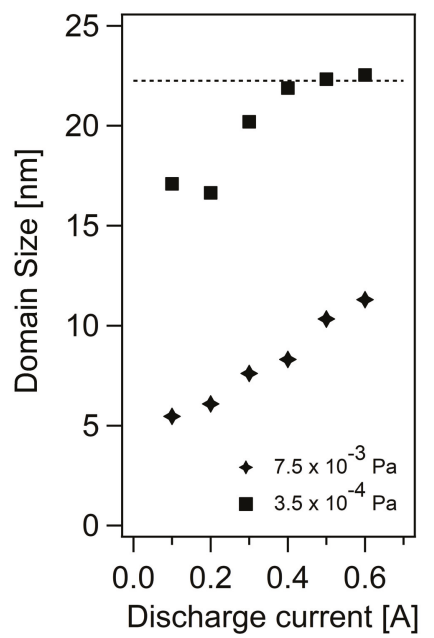

(a)

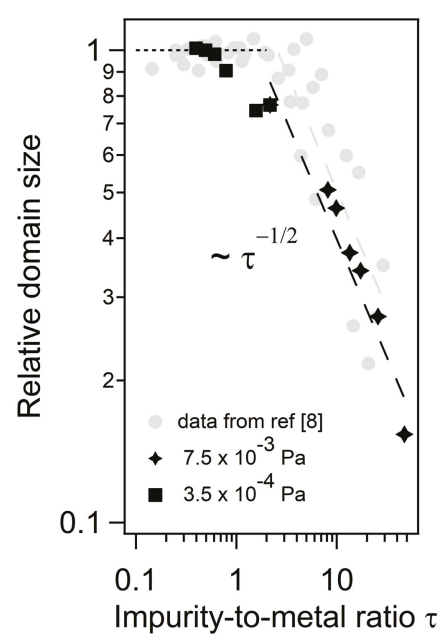

(b)

Figure 4. (a) Domain size as function of the discharge current; (b) Domain size presented as function of the impurity-to-metal impingement flux ratio. The square markers represent the deposition performed at low background pressure $\left(3.50 \times 10^{-4} \mathrm{~Pa}\right)$, the diamond markers represents the depositions performed at high background pressure $\left(7.50 \times 10^{-3} \mathrm{~Pa}\right)$. The dotted lines in the figures represents the average domain size in the low-impurity regime. The striped lines $(\mathbf{b})$ represent the relation $D \sim \tau^{-1 / 2}$.

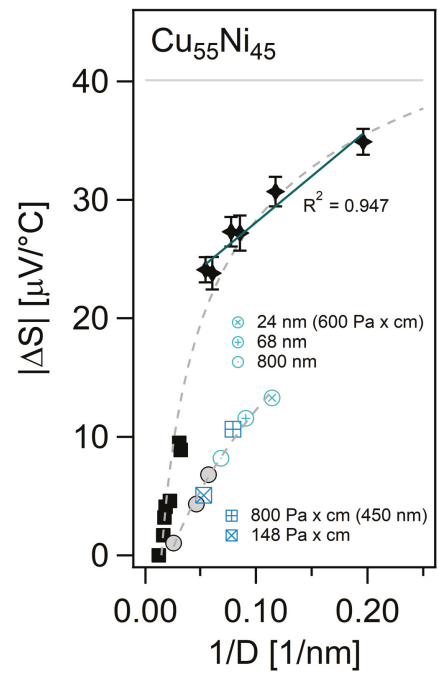

Figure 5. Deviation of the thin film Seebeck coefficient from the bulk value as function of the inverse of the domain size. The data derived from the work of Barber et al. [26] on sputter deposited constantan films are represented by the blue square unfilled markers (varying $P \times d$, constant thickness), the blue round unfilled markers (varying thickness, constant $P \times d$ ) and the round gray filled markers (both varying $P \times d$ and layer thickness). The full gray line represents the value for the maximal deviation $|\Delta S|_{\max }$. The gray dotted lines are a guide to the eye. 


\section{Discussion}

The work of Barber et al. [26] investigated the influence of the layer thickness and $P \times d$, i.e., the product of the sputter gas pressure $P$ times the target-to-substrate distance $d$, on the Seebeck coefficient of sputter deposited constantan films. The experiments showed that for a fixed $P \times d$-value, there is an increasing deviation $|\Delta S|$ from the bulk value as the film grows thinner. A similar observation was done by Gierczak et al. [27]. This is a classic observation of the size-effect. Inversely, at fixed layer thickness, $|\Delta S|$ increases with increasing $P \times d$-values. Only the data for which their respective domain size $D$ could be calculated are included in the plot in Figure 5. The influence of the layer thickness on the film Seebeck coefficient at fixed $P \times d$ is presented by the unfilled blue round markers, the influence of $P \times d$ at constant layer thickness is represented by the unfilled blue square markers. The round filled gray markers in Figure 5 represent combinations of a varying thickness together with a unspecified $P \times d$-value (thickness range $2000-90 \mathrm{~nm}$ ).

The main conclusion from the work of Barber is the direct correlation between the microstructure and the thin film Seebeck coefficient. The effect of the layer thickness was attributed to the morphological development as the film thickens. The dependency of the Seebeck coefficient on $P \times d$ is explained by thermalization effects affecting the microstructure. The role of the layer thickness is further discussed in Section 4.1, whereas the hypothesis of thermalization is confronted with our observations in Section 4.2 .

\subsection{Role of Layer Thickness}

The electrical properties of a thin metal film deviate from the bulk as soon as one of the dimensions of the metal is restricted in the order of the electron mean free path length. The mean free path length of an electron in a metal is typically of the order of tens of nanometers [28], and is therefore easily restricted by the spatial dimensions of a thin film. The effects caused by this reduced mean free path are typically called "size-effects". For example, this is a major challenge in the field of microelectronics for contacting and connection paths in the sub-50 nm range. Over time, many models have been proposed in order to describe and understand the origin of these effects. The Fuchs-Sondheimer model [29] describes the increase in thin film resistivity from the perspective of an enhanced electron scattering caused by the decreased layer thickness. The model is described as function of $l_{0} / t$, i.e., the ratio of the electron mean free path $l_{0}$ to the layer thickness $t$, and a parameter $p$, representing the fraction of specular reflected electrons at the external film surfaces (e.g., substrate-film and film-air). The thinner the film, the more electron scattering events and thus the higher the electrical resistivity of the film, i.e., $\rho \sim 1 / t$. Later on, a similar model was proposed by Mayer [30] in order to describe the thermoelectric film properties. Analogously to the Fuchs and Sondheimer description for the electrical film resistivity, Mayer predicts the deviation of the thin film Seebeck coefficient from the bulk value to scale with the inverse of the layer thickness, i.e., $\left|S_{B}-S_{F}\right|=\left|\Delta S_{F}\right| \sim l_{0} / t$. Thus according to the Mayer theory, $\Delta S_{F}$ plotted as function of $1 / t$ yields a straight line. This behavior can indeed be verified by an abundant amount of experimental data present in the literature. For example, this is reported for sputtered $\mathrm{Ni}_{90} \mathrm{Cr}_{10}$ [31], for evaporated $\mathrm{Cu}[4,18,32,33], \mathrm{Ag}$ [18,34,35], $\mathrm{Au}$ [35], Al [36], Fe [3], Sn [21], Sb [37], Bi films [37-39], or electron-beam evaporated $\mathrm{Ni}$ [40].

Sputtered thin films grow in a columnar fashion with columns growing in a lateral direction with respect to the substrate and with a mean diameter $D_{i n}$, referred here to as the in-plane domain size $[41,42]$. In most cases, the in-plane domain size is measured by means of characterization techniques such as TEM, SEM, AFM or EBSD. When a sample is measured by means of XRD, one measure the degree of crystallographic coherence in the out-of-plane direction. The more (X-ray) interference events at consecutive crystallographic planes through the thickness of the sample, the sharper the peakwidths (FWHM) of the resulting diffractogram. Using the Scherrer-equation $\left(D_{\text {out }} \sim(F W H M)^{-1}\right)$, sharp peaks with a small FWHM yield large (out-of-plane) domains $D_{\text {out }}$. For sputtered as-deposited films, the in-plane domain size $D_{\text {in }}$ measured by TEM is very similar to the out-of-plane domain size $D_{\text {out }}$ as measured by XRD [43]. Therefore, in what follows, we will use the 
out-of-plane domain size as measured by XRD as a representative measure for the in-plane domain size and both in-plane and out-of-plane are simply referred to as the domain size $D$.

For thin films grown by physical vapor deposition in general, it is an observation that the domain size increases with the film thickness, i.e., $D \sim t^{\chi}$, with $\chi$ a value between $1 / 3$ and $1 / 2$ [44-48], depending on the growth mode, substrate temperature and more. This can be verified with experimental data presented in the literature for sputtered $\mathrm{Cu}$ [49-51], Ag [52,53], Mo [42], Fe [54], FeCo [55], $\mathrm{CuNi}$ [56], $\mathrm{NiCr}$ [56] and $\mathrm{Sb}_{2} \mathrm{Te}_{3}$ [57], or for evaporated $\mathrm{Ni}_{80} \mathrm{Fe}_{20}$ (permalloy) films [58] or ion beam-evaporated Ni film [40]. Variations in film thickness are thus linked with variations in the domain size. The thinner the film, the smaller the resulting domains. Typically, for sputtered as-deposited thin films, $D<t$ [42,51-57]. Therefore, the size-depend effects observed for films with decreasing thickness can thus either be attributed to variations in $D$ or $t$ [59]. Indeed, when electrons flow through a thin film under influence of an electric field (resistivity) or temperature difference (thermoelectricity), the net flow is parallel to the substrate and film surface and thus perpendicular to the growth direction (out-of-plane) of the grains. This, in combination with the observations that $D<t$, makes it very likely that a much higher contribution of electron scattering at grain boundaries is to be expected for sputtered as-deposited films, rather than the scattering contribution from the external film surfaces as predicted by the Fuchs-Sondheimer or Mayer theory. The idea of an additional grain-boundary scattering contribution to the Fuchs-Sondheimer scattering model was already implemented under the Mayadas-Shatzkes model [41] for the description of the thin film resistivity. The shortcomings of the Fuchs-Sondheimer model to describe experimental data and the many experimental validations of the MS-model proved the dominant character of grain-boundary scattering [41,50,60,61]. Later on, Deschacht et al. [62] elaborated a similar analytical model for the description of the thermoelectric power of polycrystalline semimetal films taking into account the effects of grain boundaries. The strong contribution grain-boundary scattering could have on the Seebeck coefficient was also already hinted by other authors $[26,40,63]$. However, to our knowledge, elaborated experimental studies on the Seebeck coefficient of sputtered thin films, their relation to deposition conditions [64] and proof for the dominant character of the domain size on the Seebeck coefficient of (metallic) thin films [26] remains very limited. Based on the above discussion and in agreement with the latter work of Barber et al. [26], we conclude that the microstructural development during film growth can account for the observed variations in the film Seebeck coefficient as function of the layer thickness. In what follows, the layer thickness is kept constant $(350 \mathrm{~nm})$ for all depositions in order to exclude this influence from the discussion.

\subsection{Process Parameters}

The main effect of varying deposition conditions such as the discharge current $I$ or the argon pressure $P$ and the target-to-substrate distance $d$, is a variation in energy flux and/or material flux arriving on the substrate. As both the energy flux and the material flux are system-dependent parameters, i.e., not easily transferable towards other vacuum systems, we prefer to project them on system-objective parameters such as the energy per arriving adatom (EPA) and the impurity-to-metal impingement flux ratio $\tau$. The EPA has different energetic contributions such as a contribution from the kinetic energy of the sputtered particles or reflected neutrals, from electronic contributions, and from plasma radiation. Whereas the latter two contributions are mainly affected by the target-to-substrate distance $d$, the former two are mainly affected by $P \times d$, i.e., the product of the argon pressure and the target-to-substrate distance. As discussed in the work of Z. Barber et al. [26], $P \times d$ is a measure for the degree of thermalization. As thermalized adatoms have a restricted mobility on the substrate surface, large $P \times d$-values result in smaller grains.

In contrast to $P \times d$, the EPA remains mainly indifferent under a variation in discharge current. This because when the discharge current is decreased, both the decrease in material flux arriving on the substrate as well as the total power dissipated in the system approximately scale in a linear way [56]. The EPA is thus not significantly affected by a variation in the discharge current. The experimental 
result presented in Figure 3 is therefore not compatible with the idea of a variation in film Seebeck coefficient caused by thermalization effects. However, as the deposition speed drops with a decreasing discharge current, this causes the impurity-to-metal ratio $\tau$ to increase. It is shown in Figure 4 (right) that this latter effect is responsible for the decrease of the domain size with decreasing discharge current. Analogously, the impurity-to-metal ratio $\tau$ increases with increasing $P \times d$ as $P \times d$, and especially $d$, affects the deposition speed [56]. It can also be verified in the literature that for an increasing value for $P \times d$, the domain size decreases [65-67]. Our observations therefore indicate that a more consistent description for the effect of the discharge current on the thin film Seebeck coefficient (as presented in Figure 3) can be given from the perspective of refined grains - in coherence with the results reported in the work of Barber - but caused by an increased impurity-to-metal impingement flux $\tau$ rather than caused by thermalization effects. Furthermore, the $P \times d$-values used in the latter work, i.e., in the range of 150-800 Pa mm, are very high in comparison to the $P \times d$-value used in this work, i.e., $60 \mathrm{~Pa} \mathrm{~mm}$. The EPA values in the work of Barber are thus expected to be much lower than in this work. According to SiMTra simulations, i.e., a kinetic Monte Carlo code for the simulation of metal transport through the gas phase [68], the energy of the sputtered particles in the range of 150-800 Pa mm is estimated betzeen $1.5 \mathrm{eV}$ and $3 / 2 \mathrm{kT}$ ( $T$ at $300 \mathrm{~K}$ ) respectively and around $6 \mathrm{eV}$ for the $P \times d$-value (60 Pa $\mathrm{mm})$ used in this work. The simulations indicate fully thermalized sputtered particles, i.e., $E=3 / 2 k T$, at a $P \times d$-value close to $400 \mathrm{~Pa} \mathrm{~mm}$. Therefore, it seems less likely that the variations in $|\Delta S|$ as function of $P \times d$ (see Figure 2 in [26]) could be attributed to thermalization effects, especially for the data discussed where $P \times d>400 \mathrm{~Pa} \mathrm{~mm}$. Of course, other contributions could still contribute to the energy balance for the adatoms, although for this material-sputter gas combination, the amount of reflected neutrals is expected to be low $[69,70]$ and the electronic and radiative contributions rather scale as function of $d$ and not as $P \times d$ which is the expected dependency based on scattering events for massive particles. However, in order to demonstrate our point-of-view with respect to the importance of impurity incorporation, in the following section, both the layer thickness and the EPA are kept constant to eliminate thermalization effects and solely the impurity-to-metal ratio $\tau$ is altered.

\subsection{Impurities}

As already discussed in previous work [24], atmospheric gas impurities present in the vacuum chamber during film growth can act as grain refiners. In general, in the high-impurity regime $(\tau \gg 1)$, the average domain size $D$ decreases in a material-independent way with an increasing impurity-to-metal impingement flux ratio $\tau$ according to $D \sim \tau^{-1 / 2}$ [24]. This effect was experimentally verified in Figure 4 (right). The relation embodies the thought of a nucleation-dominated growth model. When the spatial distribution of impinging impurities on the surface is of the order of the characteristic length of the diffussing adatoms, the impurities act as active nucleation centra and restrict the adatom diffusion with refined grains as a consequence. The results presented in Figure 2 showed how the film Seebeck coefficient was strongly affected in this high-impurity regime. The similarity in behavior between the domain size $D \sim \tau^{-1 / 2}$ and the Seebeck coefficient as function of the impurity-to-metal ratio $|\Delta S| \sim \tau^{1 / 2}$ (see Figure 2) further empowers the perspective to describe the Seebeck coefficient as a film property determined by the domain size. Based on our assumptions, an approximate linear relationship $|\Delta S| \sim 1 / D$ can thus be expected in this high-impurity regime. As presented in Figure 5 (diamond markers), this prediction can be fairly well validated. Furthermore, in analogy to the work of Liu et al. [71] where the deviation in film Seebeck coefficient due to the size-effect is employed for manufacturing single-metal thermocouples, the observation of a decreased thin film Seebeck coefficient as function of $\tau$ could be exploited for creating thermocouples where both legs consist of the same material but are deposited at a different $\tau$-value.

\section{Conclusions}

The effect of impurities, layer thickness and deposition conditions such as discharge current and $P \times d$ on the thin film Seebeck coefficient is discussed. It was emphasized here how a variation in 
each of these deposition variables can affect the domain size. In view of the diversity of experimental variations which could be summarized as function of the domain size, more specifically, $1 / D$, it is justified to conclude that rather than the layer thickness, the domain size has a key role in the deviation of the thin film Seebeck coefficient from the bulk value. Therefore, the influence of deposition conditions on the Seebeck coefficient can be evaluated by understanding their effect on the domain size. This conclusion is mainly in coherence with a the work on sputter-deposited constantan by Barber et al. [26], i.e., there is a direct correlation between the microstructure and the Seebeck coefficient, but where our results indicate that the origin of the grain refinement can be attributed to an increased impurity incorporation during growth rather than to thermalization effects. Of course, it is not straightforward to discriminate between the active mechanisms as an increase in the degree of thermalization and a decrease in the deposition rate, and thus an increase in impurity-to-metal impingement flux ratio $\tau$, are unambiguously linked through an increasing target-to-substrate distance $d$. Although the data of the experiments performed in this work and in the work of Barber are quantitatively shifted in $|\Delta S|$ — which we believe can be attributed to experimental differences-this does not compromise on the generality of the discussion as the qualitative response of the Seebeck coefficient to the variations in domain size is very comparable.

Author Contributions: Conceptualization, F.G.C. and D.D.; methodology, F.G.C.; software, F.G.C.; validation, F.G.C.; formal analysis, F.G.C. and D.D.; investigation, F.G.C.; resources, F.G.C.; data curation, F.G.C.; writing-original draft preparation, F.G.C.; writing-review and editing, D.D.; visualization, F.G.C.; supervision, D.D.; project administration, D.D.; funding acquisition, D.D.

Funding: This research was funded by Ghent University through the GOA-ENCLOSE project (BOF15/GOA/007). Conflicts of Interest: The authors declare no conflict of interest.

$\begin{array}{ll}\text { Abbreviations } \\ \text { EPA } & \text { Energy per arriving adatom } \\ P & \text { Argon Pressure } \\ d & \text { Target-to-substrate distance } \\ D & \text { Domain Size } \\ t & \text { Layer thickness } \\ \text { FWHM } & \text { Full width at half maximum } \\ \text { RF } & \text { Radio-frequent } \\ \text { DC } & \text { Direct-current } \\ \text { RCA } & \text { Radio Corporation of America } \\ \text { MS } & \text { Mayadas-Shatzkes } \\ \text { PID } & \text { Proportional-integral-derivative } \\ \text { XRD } & \text { X-ray diffraction } \\ \text { TEM } & \text { Transmission electron microscope } \\ \text { AFM } & \text { Atomic force microscopy } \\ \text { EBSD } & \text { Electron backscatter diffraction }\end{array}$

\section{References}

1. Laugier, M. The construction and use of thin film thermocouples for the measurement of surface temperature: Applications to substrate temperature determination and thermal bending of a cantilevered plate during film deposition. Thin Solid Films 1980, 67, 163-170. [CrossRef]

2. Kreider, K.G. Sputtered high temperature thin film thermocouples. J. Vac. Sci. Technol. A Vac. Surf. Films 1993, 11, 1401-1405. [CrossRef]

3. Scarioni, L.; Castro, E. Thermoelectric power in thin film Fe-CuNi alloy (type-J) couples. J. Appl. Phys. 2000, 87, 4337-4339. [CrossRef]

4. Chopra, K.; Bahl, S.; Randlett, M. Thermopower in thin-film copper-Constantan couples. J. Appl. Phys. 1968, 39, 1525-1528. [CrossRef] 
5. Guo, H.; Jiang, J.Y.; Liu, J.X.; Nie, Z.H.; Ye, F.; Ma, C.F. Fabrication and Calibration of Cu-Ni Thin Film Thermocouples. Adv. Mater. Res. 2012, 512-515, 2068-2071. [CrossRef]

6. Sadat, S.; Tan, A.; Chua, Y.J.; Reddy, P. Nanoscale thermometry using point contact thermocouples. Nano Lett. 2010, 10, 2613-2617. [CrossRef] [PubMed]

7. Kim, K.; Jeong, W.; Lee, W.; Reddy, P. Ultra-high vacuum scanning thermal microscopy for nanometer resolution quantitative thermometry. Acs Nano 2012, 6, 4248-4257. [CrossRef]

8. Kim, K.; Song, B.; Fernández-Hurtado, V.; Lee, W.; Jeong, W.; Cui, L.; Thompson, D.; Feist, J.; Reid, M.H.; García-Vidal, F.J.; et al. Radiative heat transfer in the extreme near field. Nature 2015, 528, 387. [CrossRef]

9. Liu, H.; Sun, W.; Xiang, A.; Shi, T.; Chen, Q.; Xu, S. Towards on-chip time-resolved thermal mapping with micro-/nanosensor arrays. Nanoscale Res. Lett. 2012, 7, 484. [CrossRef]

10. Li, G.; Wang, Z.; Mao, X.; Zhang, Y.; Huo, X.; Liu, H.; Xu, S. Real-time two-dimensional mapping of relative local surface temperatures with a thin-film sensor array. Sensors 2016, 16, 977. [CrossRef]

11. Guk, E.; Ranaweera, M.; Venkatesan, V.; Kim, J.S. Performance and durability of thin film thermocouple array on a porous electrode. Sensors 2016, 16, 1329. [CrossRef] [PubMed]

12. Meredith, R.D.; Wrbanek, J.D.; Fralick, G.C.; Greer, L.C.; Hunter, G.W.; Chen, L. Design and operation of a fast, thin-film thermocouple probe on a turbine engine. In Proceedings of the 50th AIAA/ASME/SAE/ASEE Joint Propulsion Conference, Cleveland, OH, USA, 28-30 July 2014; p. 3923.

13. Zeng, Q.Y.; Hong, T.; Chen, L.; Cui, Y.X. Magnetron sputtering of NiCr/NiSi thin-film thermocouple sensor for temperature measurement when machining chemical explosive material. Key Eng. Mater. 2011, 467, 134-139. [CrossRef]

14. Geballe, T.; Hull, G. Seebeck effect in silicon. Phys. Rev. 1955, 98, 940. [CrossRef]

15. Gayner, C.; Kar, K.K. Recent advances in thermoelectric materials. Prog. Mater. Sci. 2016, 83, 330-382. [CrossRef]

16. Marshall, R.; Atlas, L.; Putner, T. The preparation and performance of thin film thermocouples. J. Sci. Instrum. 1966, 43, 144. [CrossRef]

17. Lin, S.F.; Leonard, W.F. Thermoelectric power of thin gold films. J. Appl. Phys. 1971, 42, 3634-3639. [CrossRef]

18. Yu, H.Y.; Leonard, W.F. Thermoelectric power of thin silver films. J. Appl. Phys. 1973, 44, 5324-5327. [CrossRef]

19. Leonard, W.F.; Yu, H. Thermoelectric power of thin copper films. J. Appl. Phys. 1973, 44, 5320-5323. [CrossRef]

20. Angadi, M.A.; Ashrit, P.V. Thermoelectric effect in ytterbium and samarium films. J. Phys. D Appl. Phys. 1981, 14, L125-L128. [CrossRef]

21. Angadi, M.; Udachan, L. Thermoelectric power measurements in thin tin films. J. Phys. D Appl. Phys. 1981, 14, L103. [CrossRef]

22. Angadi, M.A.; Shivaprasad, S.M. Thermoelectric power measurements in thin palladium films. J. Mater. Sci. Lett. 1982, 1, 65-66. [CrossRef]

23. Angadi, M.A.; Udachan, L.A. Thermopower measurements in chromium films. J. Mater. Sci. Lett. 1982, 1, 539-541. [CrossRef]

24. Cougnon, F.; Dulmaa, A.; Dedoncker, R.; Galbadrakh, R.; Depla, D. Impurity dominated thin film growth. Appl. Phys. Lett. 2018, 112, 221903. [CrossRef]

25. Guan, A.; Wang, H.; Jin, H.; Chu, W.; Guo, Y.; Lu, G. An experimental apparatus for simultaneously measuring Seebeck coefficient and electrical resistivity from $100 \mathrm{~K}$ to 600 K. Rev. Sci. Instrum. 2013, 84, 043903. [CrossRef]

26. Barber, Z.; Somekh, R. Magnetron sputtering of Cu55Ni45. Vacuum 1984, 34, 991-994. [CrossRef]

27. Gierczak, M.; Prażmowska-Czajka, J.; Dziedzic, A. Thermoelectric mixed thick-/thin film microgenerators based on constantan/silver. Materials 2018, 11, 115. [CrossRef]

28. Gall, D. Electron mean free path in elemental metals. J. Appl. Phys. 2016, 119, 085101. [CrossRef]

29. Sondheimer, E.H. The mean free path of electrons in metals. Adv. Phys. 1952, 1, 1-42. [CrossRef]

30. Mayer, H. Recent developments in conduction phenomena in thin metal films. In Structure and Properties of Thin Films; Neugebauer, C.A., Newkirk, J.W., Eds; Wiley: New York, NY, USA, 1959; p. 225.

31. Zhang, X.; Choi, H.; Datta, A.; Li, X. Design, fabrication and characterization of metal embedded thin film thermocouples with various film thicknesses and junction sizes. J. Micromech. Microeng. 2006, 16, 900. [CrossRef] 
32. Rao, V.N.; Mohan, S.; Reddy, P.J. Electrical resistivity, TCR and thermoelectric power of annealed thin copper films. J. Phys. D Appl. Phys. 1976, 9, 89. [CrossRef]

33. Thakoor, A.; Suri, R.; Suri, S.; Chopra, K. Electron transport properties of copper films. II. Thermoelectric power. J. Appl. Phys. 1975, 46, 4777-4783. [CrossRef]

34. Rao, V.N.; Mohan, S.; Reddy, P.J. The size effect in the thermoelectric power of silver films. Thin Solid Films 1977, 42, 283-289. [CrossRef]

35. Hubin, M.; Gouault, J. Resistivity and thermoelectric power between $-100{ }^{\circ} \mathrm{C}$ and $+100{ }^{\circ} \mathrm{C}$ of gold and silver thin films formed and studied in ultrahigh vacuum. Thin Solid Films 1974, 24, 311-331. [CrossRef]

36. De, D.; Bandyopadhyay, S.K.; Chaudhuri, S.; Pal, A.K. Thermoelectric power of aluminum films. J. Appl. Phys. 1983, 54, 4022-4027. [CrossRef]

37. Boyer, A.; Cisse, E. Properties of thin film thermoelectric materials: Application to sensors using the Seebeck effect. Mater. Sci. Eng. B 1992, 13, 103-111. [CrossRef]

38. Das, V.D.; Soundararajan, N. Size and temperature effects on the Seebeck coefficient of thin bismuth films. Phys. Rev. B 1987, 35, 5990. [CrossRef]

39. Mikolajczak, P.; Piasek, W.; Subotowicz, M. Thermoelectric power in bismuth thin films. Phys. Status Solidi A 1974, 25, 619-628. [CrossRef]

40. Bourque-Viens, A.; Aimez, V.; Taberner, A.; Nielsen, P.; Charette, P.G. Modelling and experimental validation of thin-film effects in thermopile-based microscale calorimeters. Sens. Actuators A Phys. 2009, 150, 199-206. [CrossRef]

41. Mayadas, A.; Shatzkes, M. Electrical-resistivity model for polycrystalline films: The case of arbitrary reflection at external surfaces. Phys. Rev. B 1970, 1, 1382. [CrossRef]

42. Hofer, A.; Schlacher, J.; Keckes, J.; Winkler, J.; Mitterer, C. Sputtered molybdenum films: Structure and property evolution with film thickness. Vacuum 2014, 99, 149-152. [CrossRef]

43. Braeckman, B.; Misják, F.; Radnóczi, G.; Caplovicova, M.; Djemia, P.; Tetard, F.; Belliard, L.; Depla, D. The nanostructure and mechanical properties of nanocomposite $\mathrm{Nb}_{x}-\mathrm{CoCrCuFeNi}$ thin films. Scr. Mater. 2017, 139, 155-158. [CrossRef]

44. Xin, Z.; Xiao-Hui, S.; Dian-Lin, Z. Thickness dependence of grain size and surface roughness for dc magnetron sputtered Au films. Chin. Phys. B 2010, 19, 086802. [CrossRef]

45. Srolovitz, D.J.; Battaile, C.C.; Li, X.; Butler, J.E. Simulation of faceted film growth in two-dimensions: Microstructure, morphology and texture. Acta Mater. 1999, 47, 2269-2281.

46. Song, X.; Liu, G. Computer simulation of normal grain growth in polycrystalline thin films. J. Mater. Sci. 1999, 34, 2433-2436. [CrossRef]

47. Thijssen, J. Simulations of polycrystalline growth in 2+1 dimensions. Phys. Rev. B 1995, 51, 1985. [CrossRef]

48. Van der Drift, A. Evolutionary selection, a principle governing growth orientation in vapour-deposited layers. Philips Res. Rep. 1967, 22, 267.

49. Barmak, K.; Darbal, A.; Ganesh, K.J.; Ferreira, P.J.; Rickman, J.M.; Sun, T.; Yao, B.; Warren, A.P.; Coffey, K.R. Surface and grain boundary scattering in nanometric $\mathrm{Cu}$ thin films: A quantitative analysis including twin boundaries. J. Vac. Sci. Technol. A Vac. Surf. Films 2014, 32, 061503. [CrossRef]

50. Sun, T.; Yao, B.; Warren, A.P.; Barmak, K.; Toney, M.F.; Peale, R.E.; Coffey, K.R. Dominant role of grain boundary scattering in the resistivity of nanometric Cu films. Phys. Rev. B 2009, 79, 041402. [CrossRef]

51. Zhang, X.; Misra, A. Residual stresses in sputter-deposited copper/330 stainless steel multilayers. J. Appl. Phys. 2004, 96, 7173-7178. [CrossRef]

52. Yu, S.; Li, L.; Lyu, X.; Zhang, W. Preparation and investigation of nano-thick FTO/Ag/FTO multilayer transparent electrodes with high figure of merit. Sci. Rep. 2016, 6, 20399. [CrossRef]

53. Ding, G.; Clavero, C. Silver-based low-emissivity coating technology for energy-saving window applications. In Modern Technologies for Creating the Thin-film Systems and Coatings; Nikitenkov, N., Ed.; InTechOpen: London, UK, 2017; pp. 409-431.

54. Shamsutdinov, N.; Sloof, W.; Böttger, A. A method for the experimental determination of surface photoemission core-level shifts for 3d transition metals. J. Appl. Phys. 2005, 98, 014908. [CrossRef]

55. Krist, T.; Teichert, A.; Meltchakov, E.; Vidal, V.; Zoethout, E.; Müllender, S.; Bijkerk, F. Stress reduction in multilayers used for X-Ray and neutron optics. In Modern Developments in X-Ray and Neutron Optics; Erko, A., Idir, M., Eds.; Springer: Heidelberg, Germany, 2008; pp. 371-388. 
56. Cougnon, F.; Schramm, I.; Depla, D. On the electrical properties of sputter deposited thin films: The role of energy and impurity flux. Thin Solid Films 2019, submitted for publication.

57. Wanarattikan, P.; Jitthammapirom, P.; Sakdanuphab, R.; Sakulkalavek, A. Effect of grain size and film thickness on the thermoelectric properties of flexible $\mathrm{Sb}_{2} \mathrm{Te}_{3}$ thin films. Adv. Mater. Sci. Eng. 2019, 2019, 6954918. [CrossRef]

58. Mayadas, A.F.; Janak, J.F.; Gangulee, A. Resistivity of Permalloy thin films. J. Appl. Phys. 1974, 45, 2780. [CrossRef]

59. Sun, T.; Yao, B.; Warren, A.P.; Barmak, K.; Toney, M.F.; Peale, R.E.; Coffey, K.R. Surface and grain-boundary scattering in nanometric Cu films. Phys. Rev. B 2010, 81, 155454. [CrossRef]

60. Zhang, W.; Brongersma, S.; Heylen, N.; Beyer, G.; Vandervorst, W.; Maex, K. Geometry effect on impurity incorporation and grain growth in narrow copper lines. J. Electrochem. Soc. 2005, 152, C832-C837. [CrossRef]

61. Birkett, M.; Penlington, R. Electrical resistivity of CuAlMo thin films grown at room temperature by dc magnetron sputtering. Mater. Res. Express 2016, 3, 075021. [CrossRef]

62. Deschacht, D.; Boyer, A.; Groubert, E. The thermoelectric power of polycrystalline semimetal films. Phys. Status Solidi A 1982, 71, K205-K209. [CrossRef]

63. Beensh-Marchwicka, G.; Osadnik, S.; Prociów, E.; Mielcarek, W. Structure and morphology of Ge(Au) sputtered films with useful Seebeck effect. Vacuum 1998, 50, 207-210. [CrossRef]

64. Arab Pour Yazdi, M.; Martin, N.; Petitot, C.; Neffaa, K.; Palmino, F.; Cherioux, F.; Billard, A. Influence of sputtering parameters on structural, electrical and thermoelectric properties of $\mathrm{Mg}$-Si coatings. Coatings 2018, 8, 380. [CrossRef]

65. Gordillo, G.; Mesa, F.; Calderón, C. Electrical and morphological properties of low resistivity Mo thin films prepared by magnetron sputtering. Braz. J. Phys. 2006, 36, 982-985. [CrossRef]

66. Chan, K.; Teo, B. Effect of Ar pressure on grain size of magnetron sputter-deposited Cu thin films. IET Sci. Meas. Technol. 2007, 1, 87-90. [CrossRef]

67. Qiu, H.; Wang, F.; Wu, P.; Pan, L.; Tian, Y. Structural and electrical properties of Cu films deposited on glass by DC magnetron sputtering. Vacuum 2002, 66, 447-452. [CrossRef]

68. Van Aeken, K.; Mahieu, S.; Depla, D. The metal flux from a rotating cylindrical magnetron: A Monte Carlo simulation. J. Phys. D Appl. Phys. 2008, 41, 205307. [CrossRef]

69. Hoffman, D.; Thornton, J.A. Internal stresses in $\mathrm{Cr}, \mathrm{Mo}$, Ta, and Pt films deposited by sputtering from a planar magnetron source. J. Vac. Sci. Technol. 1982, 20, 355-358. [CrossRef]

70. Depla, D.; Mahieu, S. Reactive Sputter Deposition; Springer: Heidelberg, Germany, 2008.

71. Liu, H.; Sun, W.; Xu, S. An extremely simple thermocouple made of a single layer of metal. Adv. Mater. 2012, 24, 3275-3279. [CrossRef]

(C) 2019 by the authors. Licensee MDPI, Basel, Switzerland. This article is an open access article distributed under the terms and conditions of the Creative Commons Attribution (CC BY) license (http:/ / creativecommons.org/licenses/by/4.0/). 

Article

\title{
Gas Sensing with Nanoplasmonic Thin Films Composed of Nanoparticles (Au, Ag) Dispersed in a $\mathrm{CuO}$ Matrix
}

\author{
Manuela Proença *, Marco S. Rodrigues, Joel Borges * and Filipe Vaz \\ Centro de Física da Universidade do Minho, Campus de Gualtar, 4710-057 Braga, Portugal; \\ marcopsr@gmail.com (M.S.R.); fvaz@fisica.uminho.pt (F.V.) \\ * Correspondence: manuelaproenca12@gmail.com (M.P.); joelborges@fisica.uminho.pt (J.B.); \\ Tel.: +351-253-510-471 (J.B.)
}

Received: 6 May 2019; Accepted: 23 May 2019; Published: 25 May 2019

\begin{abstract}
Magnetron sputtered nanocomposite thin films composed of monometallic $\mathrm{Au}$ and Ag, and bimetallic $\mathrm{Au}-\mathrm{Ag}$ nanoparticles, dispersed in a $\mathrm{CuO}$ matrix, were prepared, characterized, and tested, which aimed to find suitable nano-plasmonic platforms capable of detecting the presence of gas molecules. The Localized Surface Plasmon Resonance phenomenon, LSPR, induced by the morphological changes of the nanoparticles (size, shape, and distribution), and promoted by the thermal annealing of the films, was used to tailor the sensitivity to the gas molecules. Results showed that the monometallic films, $\mathrm{Au}: \mathrm{CuO}$ and Ag:CuO, present LSPR bands at $\sim 719$ and $\sim 393 \mathrm{~nm}$, respectively, while the bimetallic $\mathrm{Au}-\mathrm{Ag}: \mathrm{CuO}$ film has two LSPR bands, which suggests the presence of two noble metal phases. Through transmittance-LSPR measurements, the bimetallic films revealed to have the highest sensitivity to the refractive index changes, as well as high signal-to-noise ratios, respond consistently to the presence of a test gas.
\end{abstract}

Keywords: thin films; magnetron sputtering; microstructure; noble metal nanoparticles; $\mathrm{CuO}$ matrix; localized surface plasmon resonance; gas sensor

\section{Introduction}

Nanocomposite thin films, containing noble metal nanoparticles embedded in an oxide matrix, have been a subject of considerable interest for optical gas sensing due to their localized surface plasmon resonance (LSPR) properties [1,2]. Surface plasmons are coherent oscillations of free electrons excited by an electromagnetic field at the boundaries between a metal and a dielectric. They can propagate along the surface of the conductor, which are designated by surface plasmon polaritons, or be confined to metallic nanoparticles or nanostructures, in which case, are denominated as localized surface plasmons [3-5]. LSPR can give rise to strong absorption bands, the enhancement of the electromagnetic field near the nanoparticles, and the appearance of scattering to the far field [6-10]. Since its discovery, there have been significant advances in both theoretical and experimental investigations of surface plasmons, which led to the development of new modelling methods that contribute to the understanding of the morphology and to the calculation of the optical properties of nano-plasmonic systems $[8,11,12]$.

The two most well studied plasmonic metals are gold $(\mathrm{Au})$ and silver $(\mathrm{Ag})$. They exhibit LSPR bands within the visible spectrum due to the energy levels of $d$ - $d$ transitions, being used in various applications involving color $[13,14]$ as well as in sensing due to their relatively high refractive index sensitivity $[15,16]$. Since Ag nanoparticles present the sharpest and strongest bands among all metals, they are associated to higher sensitivity factors than Au. However, Au nanoparticles are more frequently selected for sensing applications due to their lower toxicity, inert nature (less prone to oxidation), and stability $[17,18]$. On the other hand, Ag-Au bimetallic nanoparticles have attracted particular attention 
due to their corresponding monometallic counterparts, which may allow further improvements on their set of properties [19-21], especially the optical behavior [16,21,22]. In fact, they are relatively easy to prepare since both metals have a face-centred cubic structure and similar lattice constants. However, it is known that the synthesis method can result in alloyed bimetallic nanoparticles [23,24], core-shell [25], and even Janus systems [26]. These features are determined by the $\mathrm{Au} / \mathrm{Ag}$ ratio in the bimetallic nanostructure, which leads to different optical properties. From the alloy formation of $\mathrm{Au}-\mathrm{Ag}$ bimetallic nanoparticles, only one LSPR band results between the peaks of the constituting monometallic nanoparticles, while a mixed system originates two plasmonic bands, as reported in different works $[3,22,23,27]$.

Diverse noble metal compositions dispersed in a dielectric matrix and different microstructures and nanostructures might be developed, which originates different LSPR bands, since their curvature and position are strongly dependent on different factors such as the composition, size, shape, and distribution of the nanoparticles, which are also sensitive to changes of the refractive index of the surrounding dielectric medium where they are dispersed [1,3]. Hence, the basis of the plasmonic bio/chemical sensors is established by the dependence of the LSPR band on the surrounding refractive index $[2,16,28]$. One of the advantages of using LSPR phenomenon for optical gas sensors in contrast to Surface Plasmon Resonance (SPR) systems is the fact that the first ones have a much higher potential to be sensitive to the extremely low refractive index changes such as those induced by gas molecules $[2,29,30]$, since the plasmon decay length in LSPR is much lower than in SPR [31]. Furthermore, LSPR-based sensors are basically supported by nanoparticles that can be directly coupled to light, while the SPR-based sensors are dependent on prisms, optical fibers, or gratings to be coupled with light $[30,32,33]$.

For the LSPR gas detection by refractive index changes to be functional, the production of highly sensitive plasmonic thin films is required, but the development of a high-resolution spectroscopy system to measure extremely small LSPR peak shifts is a fact that has been hampering the research on this area [2,34]. Hence, in order to optimize the sensitivity of the films, previous studies of the LSPR sensing response have been made by using two liquids with a relatively large refractive index difference $[28,35,36]$, which allowed us to estimate the refractive index sensitivity (RIS) [37-39].

The present work proposes a reliable and effective possibility of sensitive thin films, suitable to be used as optical sensors. Such (nanocomposite) thin films are based on $\mathrm{Au}$ and/or Ag nanoparticles, dispersed in a semiconductor copper oxide $(\mathrm{CuO})$ matrix, $\mathrm{Au}: \mathrm{CuO}, \mathrm{Ag}: \mathrm{CuO}$, and $\mathrm{Au}-\mathrm{Ag}: \mathrm{CuO}$, deposited by reactive DC magnetron sputtering. The use of a pure copper target containing gold and/or silver pellets on its surface, avoids the use of a second cathode [40,41], with evident economic advantages $[3,28]$. After the preparation of the thin films, a thermal annealing treatment was performed in order to promote the necessary nanostructural changes in the noble metal nanoparticles, and dielectric matrix, which enabled the manifestation of the LSPR behavior, and, consequently, turned the thin films sensitive to the gas molecules. The composition and morphology of the thin films were studied and correlated with the LSPR responses. LSPR sensing tests were performed through transmittance measurements in a custom-made optical vacuum system, which incorporates a gas flow cell. The sensitivity of the different films to the presence of $\mathrm{O}_{2}$ gas was also calculated and compared between them.

\section{Materials and Methods}

Thin films of $\mathrm{Au}: \mathrm{CuO}, \mathrm{Ag}: \mathrm{CuO}$, and $\mathrm{Au}-\mathrm{Ag}: \mathrm{CuO}$ manifesting LSPR behavior were produced by a two-step process, involving deposition of the thin films and posterior thermal treatment. For the depositions, two different types of substrates were used including $\mathrm{Si}$ (Boron doped, p type, $<100>$ orientation, $525 \mu \mathrm{m}$ thick) for chemical and (micro)structural characterization purposes and $\mathrm{SiO}_{2}$ (fused silica) for optical spectra measurements. Before the depositions and in order to clean and activate the surface of the substrates, plasma treatments were performed by a Low-Pressure Plasma Cleaner by Diener Electronic equipped with a $40 \mathrm{kHz}$ RF generator (Zepto Model, Ebhausen, Germany) [42], 
applying a power of $100 \mathrm{~W}$. The substrates were first cleaned with $\mathrm{O}_{2}$ plasma ( $80 \mathrm{~Pa}$, for $5 \mathrm{~min}$ ), and then activated with Ar plasma (80 Pa, for $15 \mathrm{~min}$ ).

The films were deposited by reactive (DC) magnetron sputtering during $60 \mathrm{~s}$ in order to produce films with thicknesses around $\sim 50 \mathrm{~nm}$. As illustrated in Figure 1a, the above-mentioned substrates were then placed in a grounded hexagonal holder, rotating at $16 \mathrm{rpm}$ and $7 \mathrm{~cm}$ far from the cathode. The latter is a rectangular copper target $\left(200 \times 100 \times 6 \mathrm{~mm}^{3}, 99.99 \%\right.$ purity), where gold and/or silver pellets (surface area of $960 \mathrm{~mm}^{2}$ and $0.5 \mathrm{~mm}$ thick) were placed symmetrically on its preferential erosion zone. The base pressure was below $5 \times 10^{-4} \mathrm{~Pa}$, while the target potential was limited to $500 \mathrm{~V}$, and the applied current was $3.25 \mathrm{~mA} / \mathrm{cm}^{2}$. The discharge was ignited in a gas atmosphere composed of $\operatorname{Ar}\left(3.5 \times 10^{-1} \mathrm{~Pa}\right)$ and $\mathrm{O}_{2}\left(2 \times 10^{-2} \mathrm{~Pa}\right)$. Then, in order to promote the nanoparticles' growth, the films were subjected to thermal treatments in-air, up to a maximum temperature of $700{ }^{\circ} \mathrm{C}$, according to what was previously studied and published by the group $[1,28]$. The heating ramp used was $5^{\circ} \mathrm{C} / \mathrm{min}$ and the isothermal period was $5 \mathrm{~h}$, which cooled down freely inside the furnace, before reaching room temperature.

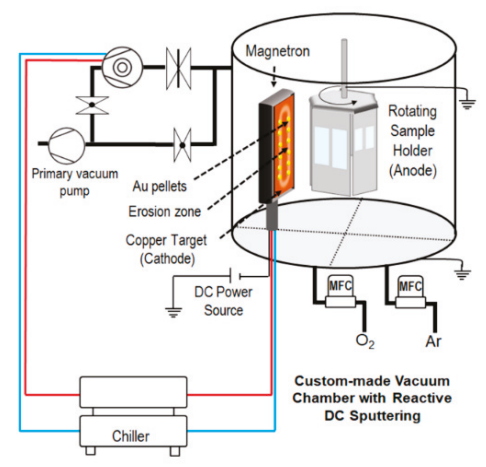

(a)

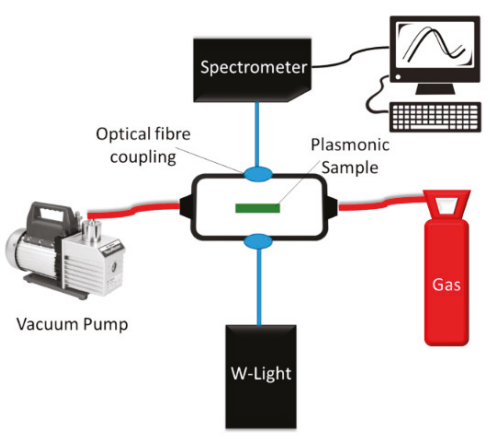

(b)

Figure 1. Simplified representation of the reactive DC magnetron sputtering system (a) and the custom-made system for transmittance-LSPR (T-LSPR) measurement in a controlled atmosphere (b).

The atomic composition of the films was studied by Rutherford Backscattering Spectrometry (RBS) using a Van de Graaff accelerator, a standard detector, placed at $140^{\circ}$, and two pin-diode detectors located symmetrically to each other, both placed at a $165^{\circ}$ scattering angle respective to the beam direction. Spectra were collected using $2.0 \mathrm{MeV} 4 \mathrm{He}^{+}$, and $1.45 \mathrm{MeV} 1 \mathrm{H}^{+}$beams at normal incidence and the data was analyzed with the IBA DataFurnace NDF v9.6i code [43].

The morphology of the films' surface was studied by a Dual Beam Scanning Electron Microscope, SEM/FIB FEI Helios 600i (Hillsboro, OR, USA), using a backscatter electron detector. The surface micrographs were analyzed using MATLAB software (version R2018a), by calculating the Feret diameter, the aspect ratio, and the nearest neighbor of the contrasted nanoparticles. The MATLAB algorithm included the locally adaptive threshold function "adaptthresh." After the binarization and scaling of the SEM images, the nanoparticles were analysed using the "regionprops" and "bwboundaries" functions.

The films' gas sensitivity was investigated by monitoring the LSPR band in the presence of $\mathrm{O}_{2}$ (atmospheric pressure), in comparison to a low vacuum pressure. Real-time measurements were performed in a custom-made system (Figure 1b), composed of two main parts: the optical components and a vacuum system. The optical system allows the measurement of the optical (transmittance) spectrum of the sample, using a tungsten lamp and a modular spectrometer by Ocean Optics (HR4000, Edinburgh, UK). Optical fibers were used to connect those components to the flow cell, where the sample is placed. A vacuum pump was used to produce a "primary" vacuum ( $40 \mathrm{~Pa})$ inside the flow cell and then $\mathrm{O}_{2}$ was introduced at atmospheric pressure for $120 \mathrm{~s}$. Several vacuum $/ \mathrm{O}_{2}$ cycles were 
employed and the LSPR peak position was monitored in real time. A MATLAB algorithm was written to smooth the spectra and find the position of the LSPR peak over time.

\section{Results}

\subsection{Thin Films Characterization}

The atomic concentration profiles of the thin films were determined by RBS (Figure 2). The as-deposited $\mathrm{CuO}$ matrix (solid lines), and the $\mathrm{CuO}$ matrix with thermal treatment at $700{ }^{\circ} \mathrm{C}$ (dash lines), are represented in Figure 2a, while the as-deposited nanocomposite films are displayed in Figure $2 b-d$. According to the RBS analysis, all the as-deposited thin films were found to have a roughly constant atomic concentration across their thickness, even after the annealing process for the case of the pure matrix. Moreover, elemental concentration results revealed that the matrix of the as-deposited films is not fully $\mathrm{CuO}$ stoichiometric, since the atomic ratio $C_{\mathrm{O}} / C_{\mathrm{Cu}}$ is always different from but close to 1 . However, as soon as the film is subjected to thermal annealing, it seems that the $\mathrm{CuO}$ matrix becomes stoichiometric, which can be observed by the corresponding RBS profile (Figure 2a), where $\mathrm{Cu}$ and $\mathrm{O}$ concentrations were estimated to be about $50.0 \pm 0.5$ at. $\%$ and $50 \pm 3$ at. $\%$, respectively. Thus, when the films are subjected to thermal treatment in air, the chemical composition may change in relation to the as-deposited films due to oxygen incorporation [44,45], as previously verified [1,28]. The atomic concentration of noble metals into the $\mathrm{CuO}$ matrix was determined to be about $C_{\mathrm{Au}}=15.0 \pm 0.5$ at. $\%$ $(\mathrm{Au}: \mathrm{CuO}), C_{\mathrm{Ag}}=17.7 \pm 0.5$ at.\% $(\mathrm{Ag}: \mathrm{CuO})$, and $C_{\mathrm{Au}}=6.7 \pm 0.5$ at.\%, $C_{\mathrm{Ag}}=8.0 \pm 0.5$ at. $\%(\mathrm{Au}-\mathrm{Ag}: \mathrm{CuO})$. These were the compositions of the thin films used for LSPR sensitivity tests.

(a)

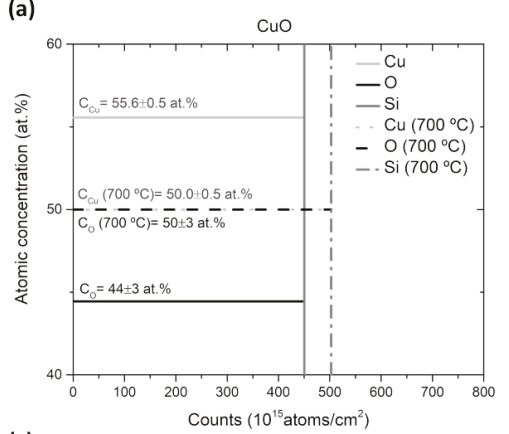

(c)

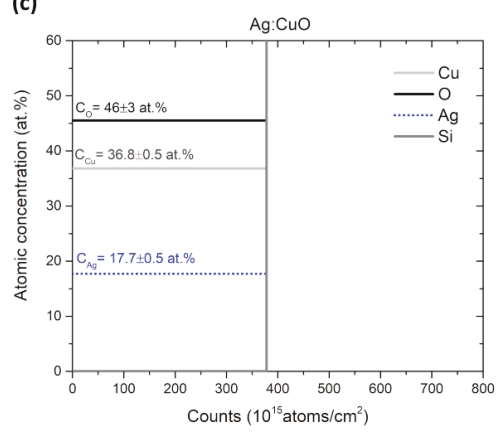

(b)

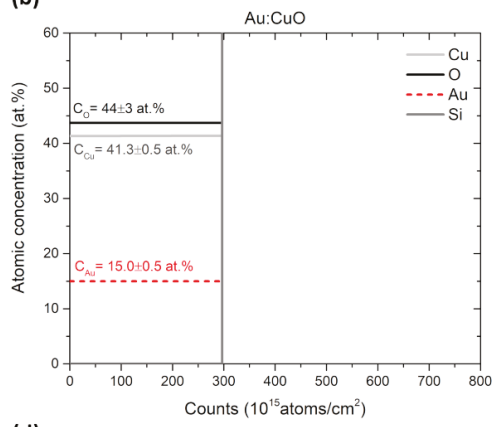

(d)

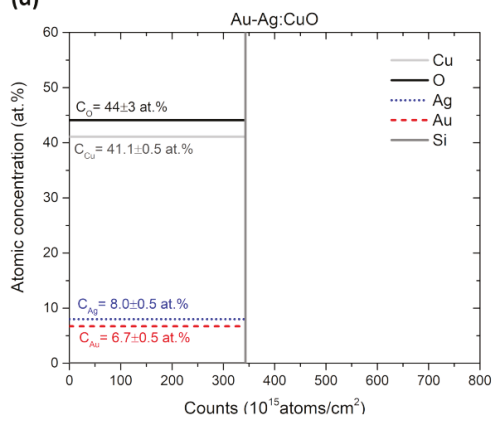

Figure 2. Atomic concentration (at.\%) of the different elements present in the as-deposited CuO matrix (solid lines (a)), in the $\mathrm{CuO}$ matrix with annealing at $700{ }^{\circ} \mathrm{C}$ (dash lines (a)), and in the as-deposited samples of $\mathrm{Au}: \mathrm{CuO}(\mathbf{b}), \mathrm{Ag}: \mathrm{CuO}(\mathbf{c})$, and $\mathrm{Au}-\mathrm{Ag}: \mathrm{CuO}(\mathrm{d})$ films deposited with a pellets' area of $960 \mathrm{~mm}^{2}$, obtained by the RBS data analyzed with the code IBA DataFurnace NDF v9.6i [43]. 
The $\mathrm{CuO}$ matrix annealed at $700{ }^{\circ} \mathrm{C}$ presents a polycrystalline structure with well-defined grain boundaries, as observed in the SEM micrograph displayed in Figure 3a. In addition, the optical transmittance spectrum (Figure $3 \mathrm{~b}$ ) reveals a semi-transparent $\mathrm{CuO}$ matrix in the visible range, with a progressive increase of transmittance for higher wavelengths, which is a feature that is in agreement with the literature [46].

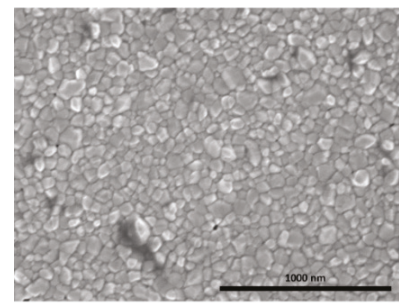

(a)

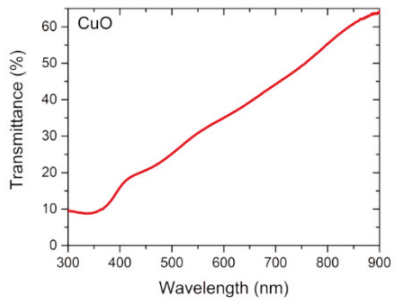

(b)

Figure 3. Top-view SEM micrograph of the $\mathrm{CuO}$ matrix annealed at $700{ }^{\circ} \mathrm{C}(\mathbf{a})$ and the respective optical transmittance spectrum (b).

The microstructural analysis of the annealed plasmonic thin films revealed the presence of noble nanoparticles (bright spots) in the different nanocomposite thin films ((a) and (b) in Figures 4-6), which suggests that the growth of nanoparticles might be facilitated by easier diffusion of $\mathrm{Au}$ and $\mathrm{Ag}$ atoms through grain boundaries of the $\mathrm{CuO}$ matrix. The $\mathrm{Au}: \mathrm{CuO}$ (Figure 4) film is the one that presents the highest nanoparticles' density at the surface $\left(127 \mu^{-2}\right)$ with an average size of about $33 \mathrm{~nm}$ (Figure 4c). Moreover, the Au nanoparticles are relatively close to each other (Figure $4 \mathrm{~d}$ ) and they are presumably spherical since their aspect ratio distribution is narrow and close to 1, as seen in Figure 4e.

(a)

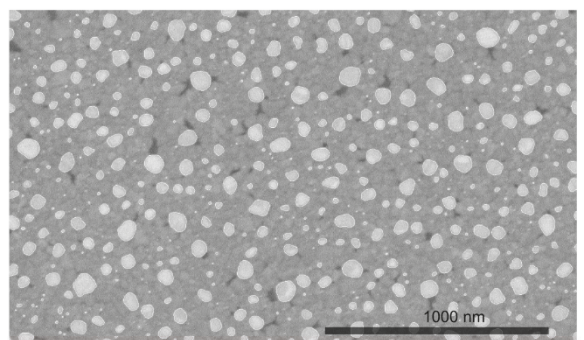

(c)

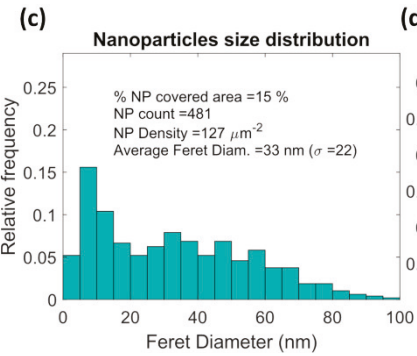

(d) (b) Thresholded image

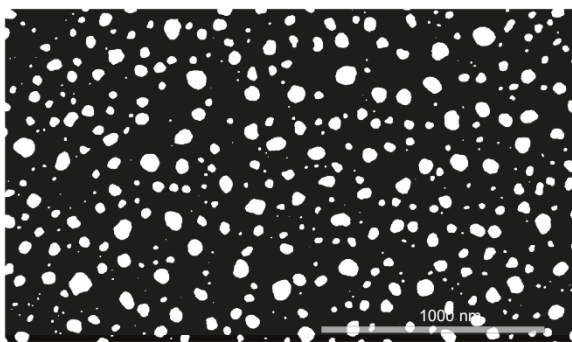

(e)

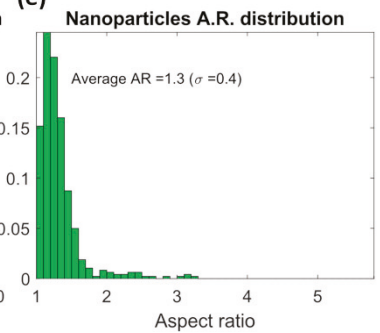

Figure 4. Au nanoparticle distribution analysis, using a MATLAB algorithm: (a) top-view SEM micrograph with atomic weight contrast and (b) processed and threshold micrograph showing the exposed Au nanoparticles, with 15\% Au coverage area. Distribution histograms are displayed in (c) for the nanoparticles Feret diameter, (d) for the nearest neighbor distance, and (e) for the aspect ratio. 
Regarding the Ag:CuO film (Figure 5), the average size of Ag nanoparticles was estimated to be $15 \mathrm{~nm}$. However, the nanoparticles' density at the surface $\left(69 \mu \mathrm{m}^{2}\right)$ is much smaller than in the other films (Figure 5c), which leads to the highest distance between the nanoparticles (Figure 5d). In fact, the formation of islands of $\mathrm{Ag}$ (micro-sized agglomerates with parallelepiped shape) was observed on the surface of the film (not shown here) [28]. This explains the low amount of nanosized Ag particles, which is a behavior that was not expected when taking into account the relatively high Ag atomic concentration determined for the as-deposited film.

The Au-Ag:CuO film (Figure 6) presents values between those belonging to the monometallic counterparts (Figures 4 and 5). It presents a density of Au-Ag nanoparticles at the surface of $100 \mu \mathrm{m}^{-2}$, with an average size estimated to be $30 \mathrm{~nm}$ (Figure 6c). Moreover, the nearest neighbor distance distribution is broader than in the Au film and narrower than in the $\mathrm{Ag}$ film. Moreover, this system shows the widest aspect ratio distribution, with an average value of 1.5 , which proves that both spherical and irregular nanoparticles are present in the film's surface.

The different microstructures achieved by the films with the thermal treatment originated different optical transmittance responses, as shown in Figure 7. The high Au nanoparticles' density at the surface and their quasi-spherical shape, observed in the $\mathrm{Au}: \mathrm{CuO}$ film (Figure 4), gave rise to a well-defined and sharp transmittance LSPR (T-LSPR) band at $\sim 719 \mathrm{~nm}$ (Figure 7a), with a high transmittance amplitude, at about 15 percentage points (i.e., the difference between the maximum and the minimum band's peak).

On the other hand, a T-LSPR band was also observed for the Ag:CuO film (Figure 7b), appearing at shorter wavelengths $(\sim 393 \mathrm{~nm})$ as is typical of the Ag nanoparticles [3,28]. However, despite the narrow shape, due to its slightly larger nanoparticle aspect ratio distribution, the LSPR band is also less intense since the number of Ag nanoparticles at the surface is scarce, which presents only a transmittance amplitude of $\sim 10$ percentage points.

(a)

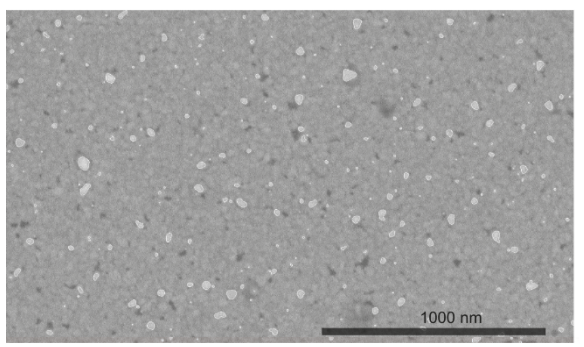

(c)
Backscattered electrons

(d) (b)

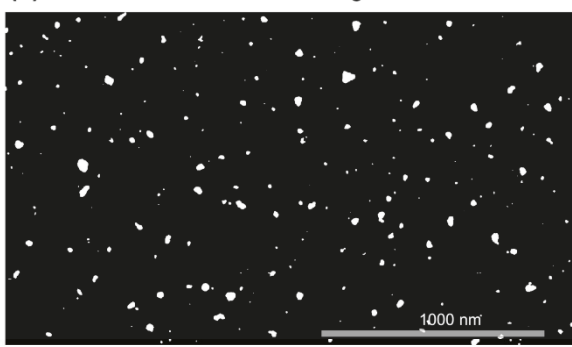

(e)
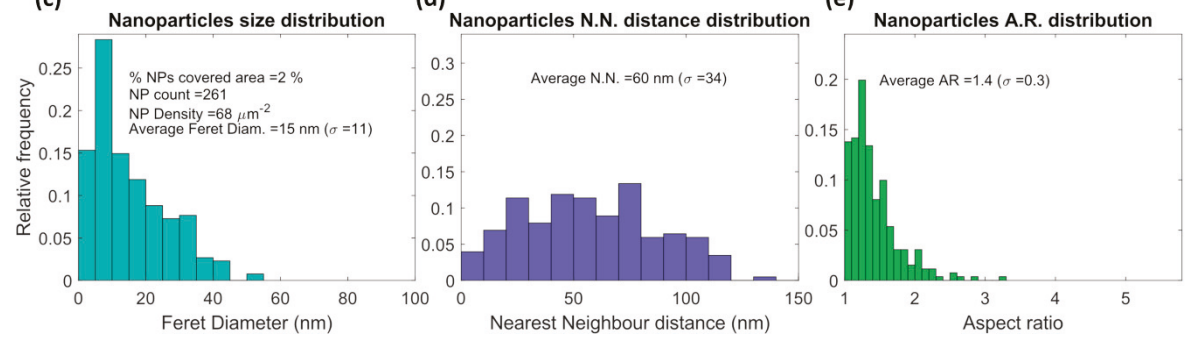

Figure 5. Ag nanoparticle distribution analysis, using a MATLAB algorithm: (a) top-view SEM micrograph with atomic weight contrast and (b) processed and threshold micrograph showing the exposed Ag nanoparticles, with 2\% Ag coverage area. Distribution histograms are displayed in (c) for nanoparticles Feret diameter, (d) for the nearest neighbor distance, and (e) for the aspect ratio. 
(a)

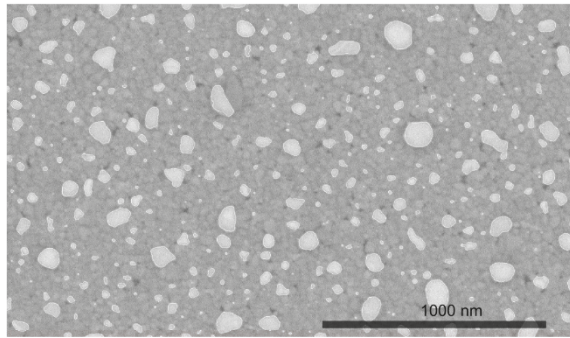

(c)

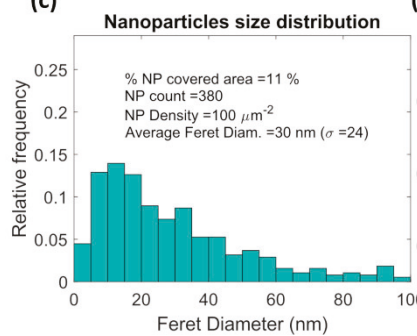

(d) (b)

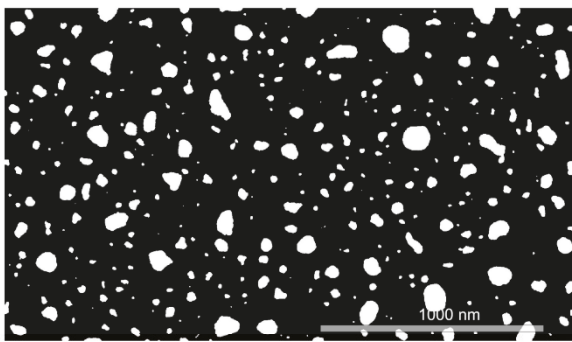

(e) Nanoparticles N.N. distance distribution
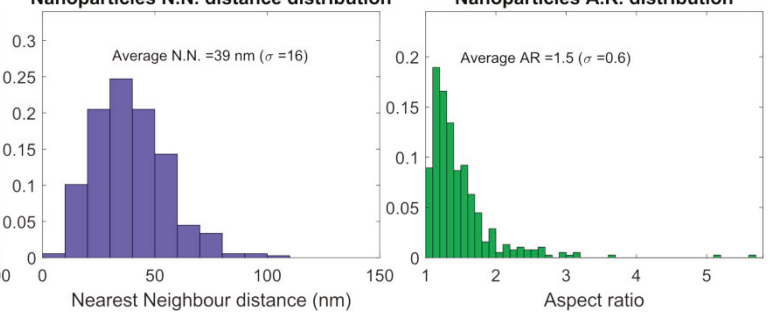

Figure 6. Au-Ag nanoparticle distribution analysis, using a MATLAB algorithm: (a) top-view SEM micrograph with atomic weight contrast and (b) processed and threshold micrograph showing the exposed Au-Ag nanoparticles, with 11\% Au-Ag coverage area. Distribution histograms are displayed in (c) for nanoparticles Feret diameter, (d) for the nearest neighbor distance, and (e) for the aspect ratio.

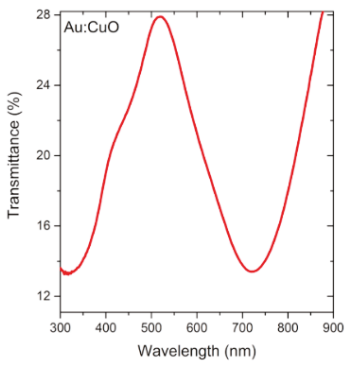

(a)

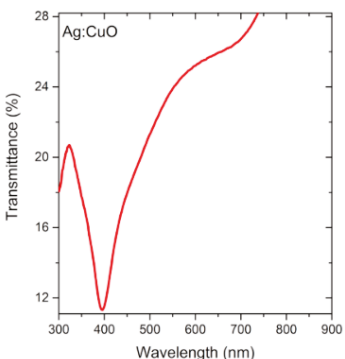

(b)

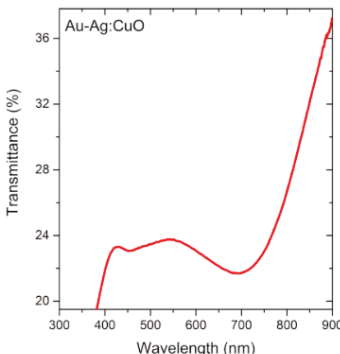

(c)

Figure 7. Transmittance spectra of the $\mathrm{Au}: \mathrm{CuO}(\mathbf{a}), \mathrm{Ag}: \mathrm{CuO}(\mathbf{b})$, and $\mathrm{Au}-\mathrm{Ag}: \mathrm{CuO}$ (c) thin films after in-air annealing.

Concerning the Au-Ag:CuO film, two shifted LSPR peaks are observed ( 450 and $676 \mathrm{~nm}$ ), even though the second one is much more pronounced (Figure 7c). The presence of two peaks might suggest the presence of separate phases of $\mathrm{Ag}$ and Au nanoparticles in these films, but since they are shifted from their initial positions, the formation of an alloy of Au-Ag bimetallic nanoparticles cannot be disregarded. Furthermore, as observed in Figure 6, the film presents both spherical and elongated nanoparticles, which contribute to the LSPR band widening and, therefore, appears much less intense.

\subsection{Sensitivity Tests Using Exposure to $\mathrm{O}_{2}$}

In order to test the films' sensitivity to refractive index changes promoted by the presence and/or adsorption of gas molecules, they were exposed to a test gas $\left(\mathrm{O}_{2}\right)$. Figure 8 presents the LSPR peak position (transmittance) of the three systems, during five cycles under vacuum, and $\mathrm{O}_{2}$ at atmospheric pressure. As expected from this type of sensor, the transmittance shift due to a change in the refractive 
index is typically very short, in the order of tenths of percentage points [2,47]. Anyway, it is possible to observe that the films responded consistently to the presence of the gas. The T-LSPR peak shifted to lower transmittances when the $\mathrm{O}_{2}$ was introduced, which decreases by $0.35,0.11$, and 0.43 percentage points for the $\mathrm{Au}, \mathrm{Ag}$, and $\mathrm{Au}-\mathrm{Ag}: \mathrm{CuO}$ films, respectively. These results are consistent with what has been already published for $\mathrm{Au}-\mathrm{TiO}_{2}$ films, but with slightly higher sensitivities [2]. The Ag:CuO sample presents the lowest shift and, subsequently, the lowest signal-to-noise ratio $(\sim 3)$. This is believed to result from the morphology achieved after the annealing process (Figure 5), where the presence of Ag nanoparticles at the film's surface is scarce, which might hinder the film's sensitivity. Moreover, the presence of $\mathrm{Au}$ in the $\mathrm{Au}: \mathrm{CuO}$ film and both $\mathrm{Ag}$ and $\mathrm{Au}$ nanoparticles in Au-Ag:CuO film seems to improve the film's response since a higher transmittance shift is observed when the test gas is introduced. In addition to show the highest transmittance shift, the Au-Ag:CuO film also presents the best signal-to-noise ratio ( 123) even though the Au:CuO film has also a reasonable value of $\sim 59$. Furthermore, the peak shifts are reproducible every cycle when the test gas is introduced, which suggests that the eventual gas adsorption is reversible.

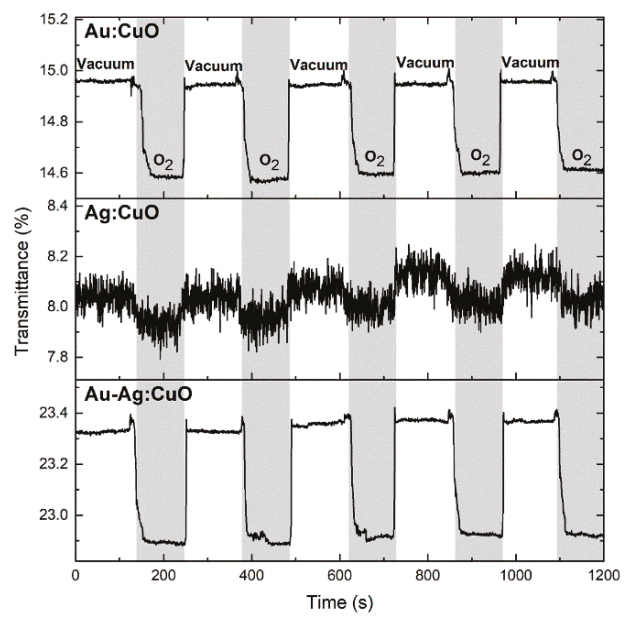

Figure 8. Variation of the LSPR peak position (transmittance minimum) of the $\mathrm{Au}: \mathrm{CuO}, \mathrm{Ag}: \mathrm{CuO}$, and $\mathrm{Au}-\mathrm{Ag}: \mathrm{CuO}$ films over time for five cycles of vacuum and $\mathrm{O}_{2}$ atmosphere.

\section{Conclusions}

$\mathrm{Au}: \mathrm{CuO}, \mathrm{Ag}: \mathrm{CuO}$, and $\mathrm{Au}-\mathrm{Ag}: \mathrm{CuO}$ thin films with nanoplasmonic properties were produced in this work. The films were first deposited by magnetron sputtering for $1 \mathrm{~min}$, using a Cu target with small metallic pellets $\left(960 \mathrm{~mm}^{2}\right.$ pellet area) and a target potential limited to $500 \mathrm{~V}$. Then, the different films were annealed up to $700{ }^{\circ} \mathrm{C}$ in order to promote the nanoparticles' growth and structural changes.

The composition analysis revealed the presence of reasonable amounts of noble metals in a $\mathrm{CuO}$ matrix, which becomes stoichiometric after a thermal treatment in air. Furthermore, the annealing induced structural and morphological changes that influenced the LSPR responses of the thin films. Due to the presence of spherical Au nanoparticles with high density at the surface, the Au:CuO film presented the most well-defined and pronounced transmittance LSPR band at $\sim 19 \mathrm{~nm}$, while the Ag:CuO film showed a narrower but less intense band at shorter wavelengths $(\sim 393 \mathrm{~nm})$ due to the scarce number of Ag nanoparticles at the surface. However, despite the fact that the $\mathrm{Au}-\mathrm{Ag}: \mathrm{CuO}$ film has two T-LSPR peaks ( 450 and $676 \mathrm{~nm})$ with relatively low intensity, it showed to be the most sensitive to the refractive index changes, such as to the $\mathrm{O}_{2}$ gas presence, followed after by $\mathrm{Au}: \mathrm{CuO}$ and Ag:CuO films. 
In conclusion, this work proves that the sensitivity of $\mathrm{Au}-\mathrm{Ag}: \mathrm{CuO}$ thin films to the test gas $\left(\mathrm{O}_{2}\right)$ can be improved by preparing bimetallic noble nanoparticles embedded in the $\mathrm{CuO}$ matrix. Hence, this configuration might be preferable to use for LSPR gas sensing.

Author Contributions: Conceptualization, J.B., F.V.; methodology, M.S.R., J.B.; software, M.S.R.; validation, M.P., M.S.R., J.B., F.V.; formal analysis, M.P., M.S.R., J.B., F.V.; investigation, M.P., M.S.R., J.B.; resources, J.B., F.V.; data curation, J.B., M.S.R., F.V.; writing—original Draft preparation, M.P., J.B.; writing—review and Editing, J.B., F.V.; visualization, M.P., M.S.R., J.B., F.V.; supervision, J.B., F.V.; project administration, F.V.; funding acquisition, J.B., F.V.

Funding: This research was funded by the Portuguese Foundation for Science and Technology (FCT) in the framework of the Strategic Funding UID/FIS/04650/2019; and by the projects NANOSENSING POCI-01-0145-FEDER-016902, with FCT reference PTDC/FIS-NAN/1154/2014; and project NANO4BIO POCI-01-0145-FEDER-032299, with FCT reference PTDC/FIS-MAC/32299/2017 supported this work. Manuela Proença acknowledges her PhD Scholarship from FCT, with reference SFRH/BD/137076/2018. Joel Borges acknowledges FCT for his Researcher Contract from project NANO4BIO, CTTI-149/18-CF(1). Marco S. Rodrigues acknowledges FCT for his PhD Scholarship, SFRH/BD/118684/2016.

Conflicts of Interest: The authors declare no conflict of interest.

\section{References}

1. Proença, M.; Borges, J.; Rodrigues, M.S.; Domingues, R.P.; Dias, J.P.; Trigueiro, J.; Bundaleski, N.; Teodoro, O.M.N.D.; Vaz, F. Development of $\mathrm{Au} / \mathrm{CuO}$ nanoplasmonic thin films for sensing applications. Surf. Coat. Technol. 2018, 343, 178-185. [CrossRef]

2. $\quad$ Rodrigues, M.S.; Borges, J.; Proença, M.; Pedrosa, P.; Martin, N.; Romanyuk, K.; Kholkin, A.L.; Vaz, F. Nanoplasmonic response of porous $\mathrm{Au}-\mathrm{TiO}_{2}$ thin films prepared by oblique angle deposition. Nanotechnology 2019, 30, 22. [CrossRef]

3. Borges, J.; Ferreira, C.G.; Fernandes, J.P.C.; Rodrigues, M.S.; Proença, M.; Apreutesei, M.; Alves, E.; Barradas, N.P.; Moura, C.; Vaz, F. Thin films of Ag-Au nanoparticles dispersed in $\mathrm{TiO}_{2}$ : Influence of composition and microstructure on the LSPR and SERS responses. J. Phys. D. Appl. Phys. 2018, 51, 205102. [CrossRef]

4. $\quad$ Borges, J.; Kubart, T.; Kumar, S.; Leifer, K.; Rodrigues, M.S.; Duarte, N.; Martins, B.; Dias, J.P.; Cavaleiro, A.; Vaz, F. Microstructural evolution of $\mathrm{Au} / \mathrm{TiO}_{2}$ nanocomposite films: The influence of Au concentration and thermal annealing. Thin Solid Films 2015, 580, 77-88. [CrossRef]

5. Borges, J.; Pereira, R.M.S.; Rodrigues, M.S.; Kubart, T.; Kumar, S.; Leifer, K.; Cavaleiro, A.; Polcar, T.; Vasilevskiy, M.I.; Vaz, F. Broadband optical absorption caused by the plasmonic response of coalesced Au nanoparticles embedded in a $\mathrm{TiO}_{2}$ matrix. J. Phys. Chem. C 2016, 120, 16931-16945. [CrossRef]

6. Ghosh, S.K.; Pal, T. Interparticle coupling effect on the surface plasmon resonance of gold nanoparticles: From theory to applications. Chem. Rev. 2007, 107, 4797-4862. [CrossRef]

7. Hutter, E.; Fendler, J.H. Exploitation of localized surface plasmon resonance. Adv. Mater. 2004, 16, 1685-1706. [CrossRef]

8. Toudert, J.; Simonot, L.; Camelio, S.; Babonneau, D. Advanced optical effective medium modeling for a single layer of polydisperse ellipsoidal nanoparticles embedded in a homogeneous dielectric medium: Surface plasmon resonances. Phys. Rev. B 2012, 86, 045415. [CrossRef]

9. Politano, A.; Formoso, V.; Chiarello, G. Dispersion and damping of gold surface plasmon. Plasmonics 2008, 3, 165-170. [CrossRef]

10. Pitarke, J.M.; Silkin, V.M.; Chulkov, E.V.; Echenique, P.M. Theory of surface plasmons and surface-plasmon polaritons. Rep. Prog. Phys. 2007, 70, 1. [CrossRef]

11. Scholl, J.A.; Koh, A.L.; Dionne, J.A. Quantum plasmon resonances of individual metallic nanoparticles. Nature 2012, 483, 421-427. [CrossRef]

12. Goyenola, C.; Gueorguiev, G.K.; Stafström, S.; Hultman, L. Fullerene-like CS $x$ : A first-principles study of synthetic growth. Chem. Phys. Lett. 2011, 506, 86-91. [CrossRef]

13. Rodrigues, M.S.; Borges, J.; Gabor, C.; Munteanu, D.; Apreutesei, M.; Steyer, P.; Lopes, C.; Pedrosa, P.; Alves, E.; Barradas, N.P.; et al. Functional behaviour of $\mathrm{TiO}_{2}$ films doped with noble metals. Surf. Eng. 2016, 32, 554-561. [CrossRef] 
14. Torrell, M.; Cunha, L.; Cavaleiro, A.; Alves, E.; Barradas, N.P.; Vaz, F. Functional and optical properties of $\mathrm{Au}: \mathrm{TiO}_{2}$ nanocomposite films: The influence of thermal annealing. Appl. Surf. Sci. 2010, 256, 6536-6542. [CrossRef]

15. Zhao, Y.; Yang, Y.; Cui, L.; Zheng, F.; Song, Q. Electroactive Au@Ag nanoparticles driven electrochemical sensor for endogenous $\mathrm{H}_{2} \mathrm{~S}$ detection. Biosens. Bioelectron. 2018, 117, 53-59. [CrossRef] [PubMed]

16. Ghodselahi, T.; Arsalani, S.; Neishaboorynejad, T. Synthesis and biosensor application of Ag@Au bimetallic nanoparticles based on localized surface plasmon resonance. Appl. Surf. Sci. 2014, 301, 230-234. [CrossRef]

17. Borges, J.; Buljan, M.; Sancho-Parramon, J.; Bogdanovic-Radovic, I.; Siketic, Z.; Scherer, T.; Kübel, C.; Bernstorff, S.; Cavaleiro, A.; Vaz, F.; et al. Evolution of the surface plasmon resonance of Au:TiO 2 nanocomposite thin films with annealing temperature. J. Nanopart. Res. 2014, 16, 2790. [CrossRef]

18. Petryayeva, E.; Krull, U.J. Localized surface plasmon resonance: Nanostructures, bioassays and biosensing-A review. Anal. Chim. Acta 2011, 706, 8-24. [CrossRef] [PubMed]

19. Cesca, T.; Michieli, N.; Kalinic, B.; Balasa, I.G.; Rangel-Rojo, R.; Reyes-Esqueda, J.A.; Mattei, G. Bidimensional ordered plasmonic nanoarrays for nonlinear optics, nanophotonics and biosensing applications. Mater. Sci. Semicond. Process. 2019, 92, 2-9. [CrossRef]

20. Dwivedi, C.; Chaudhary, A.; Srinivasan, S.; Nandi, C.K. Polymer stabilized bimetallic alloy nanoparticles: Synthesis and catalytic application. Colloid Interface Sci. Commun. 2018, 24, 62-67. [CrossRef]

21. Khlebtsov, B.N.; Liu, Z.; Ye, J.; Khlebtsov, N.G. Au@Ag core/shell cuboids and dumbbells: Optical properties and SERS response. J. Quant. Spectrosc. Radiat. Transf. 2015, 167, 64-75. [CrossRef]

22. Sangpour, P.; Akhavan, O.; Moshfegh, A.Z. The effect of Au/Ag ratios on surface composition and optical properties of co-sputtered alloy nanoparticles in Au-Ag: $\mathrm{SiO}_{2}$ thin films. J. Alloy. Compd. 2009, 486, 22-28. [CrossRef]

23. Sangpour, P.; Akhavan, O.; Moshfegh, A.Z. rf reactive co-sputtered Au-Ag alloy nanoparticles in $\mathrm{SiO}_{2}$ thin films. Appl. Surf. Sci. 2007, 253, 7438-7442. [CrossRef]

24. Hareesh, K.; Joshi, R.P.; Sunitha, D.V.; Bhoraskar, V.N.; Dhole, S.D. Anchoring of Ag-Au alloy nanoparticles on reduced graphene oxide sheets for the reduction of 4-nitrophenol. Appl. Surf. Sci. 2016, 389, 1050-1055.

25. Tiunov, I.A.; Gorbachevskyy, M.V.; Kopitsyn, D.S.; Kotelev, M.S.; Ivanov, E.V.; Vinokurov, V.A.; Novikov, A.A. Synthesis of large uniform gold and core-shell gold-silver nanoparticles: Effect of temperature control. Russ. J. Phys. Chem. A 2016, 90, 152-157. [CrossRef]

26. Song, Y.; Liu, K.; Chen, S. AgAu bimetallic janus nanoparticles and their electrocatalytic activity for oxygen reduction in alkaline media. Langmuir 2012, 28, 17143-17152. [CrossRef] [PubMed]

27. Blaber, M.G.; Arnold, M.D.; Harris, N.; Ford, M.J.; Cortie, M.B. Plasmon absorption in nanospheres: A comparison of sodium, potassium, aluminium, silver and gold. Phys. B Condens. Matter 2007, 394, 184-187. [CrossRef]

28. Proença, M.; Borges, J.; Rodrigues, M.S.; Meira, D.I.; Sampaio, P.; Dias, J.P.; Pedrosa, P.; Martin, N.; Bundaleski, N.; Teodoro, O.M.N.D.; et al. Nanocomposite thin films based on Au-Ag nanoparticles embedded in a $\mathrm{CuO}$ matrix for localized surface plasmon resonance sensing. Appl. Surf. Sci. 2019, 484, 152-168. [CrossRef]

29. Honda, M.; Ichikawa, Y.; Rozhin, A.G.; Kulinich, S.A. UV plasmonic device for sensing ethanol and acetone. Appl. Phys. Express 2018, 11, 012001. [CrossRef]

30. Kreno, L.E.; Hupp, J.T.; Van Duyne, R.P. Metal-organic framework thin film for enhanced localized surface Plasmon resonance gas sensing. Anal. Chem. 2010, 82, 8042-8046. [CrossRef]

31. Sagle, L.B.; Ruvuna, L.K.; Ruemmele, J.A.; Van Duyne, R.P. Advances in localized surface plasmon resonance spectroscopy biosensing. Nanomedicine 2011, 6, 1447-1462. [CrossRef] [PubMed]

32. Hammond, J.L.; Bhalla, N.; Rafiee, S.D.; Estrela, P. Localized surface plasmon resonance as a biosensing platform for developing countries. Biosensors 2014, 4, 172-188. [CrossRef] [PubMed]

33. Haes, A.J.; Zou, S.; Schatz, G.C.; Van Duyne, R.P. Nanoscale optical biosensor: Short range distance dependence of the localized surface plasmon resonance of noble metal nanoparticles. J. Phys. Chem. B 2004, 108, 6961-6968. [CrossRef]

34. Bingham, J.M.; Anker, J.N.; Kreno, L.E.; Duyne, R.P. Van gas sensing with high-resolution localized surface plasmon resonance spectroscopy. J. Am. Chem. Soc. 2010, 132, 17358-17359. [CrossRef] 
35. Demirdjian, B.; Bedu, F.; Ranguis, A.; Ozerov, I.; Henry, C.R. Water adsorption by a sensitive calibrated gold plasmonic nanosensor. Langmuir 2018, 34, 5381-5385. [CrossRef]

36. Jeong, H.H.; Mark, A.G.; Alarcón-Correa, M.; Kim, I.; Oswald, P.; Lee, T.C.; Fischer, P. Dispersion and shape engineered plasmonic nanosensors. Nat. Commun. 2016, 7, 11331. [CrossRef] [PubMed]

37. Chen, P.; Liedberg, B. Curvature of the localized surface plasmon resonance peak. Anal. Chem. 2014, 86, 7399-7405. [CrossRef]

38. Kedem, O.; Vaskevich, A.; Rubinstein, I. Critical issues in localized plasmon sensing. J. Phys. Chem. C 2014, 118, 8227-8244. [CrossRef]

39. Jung, L.S.; Campbell, C.T.; Chinowsky, T.M.; Mar, M.N.; Yee, S.S. Quantitative interpretation of the response of surface plasmon resonance sensors to adsorbed films. Langmuir 1998, 14, 5636-5648. [CrossRef]

40. Das, S.; Alford, T.L. Structural and optical properties of Ag-doped copper oxide thin films on polyethylene napthalate substrate prepared by low temperature microwave annealing. J. Appl. Phys. 2013, 113, 244905. [CrossRef]

41. Rydosz, A.; Szkudlarek, A. Gas-sensing performance of M-doped CuO-based thin films working at different temperatures upon exposure to propane. Sensors 2015, 15, 20069-20085. [CrossRef]

42. Pedrosa, P.; Fiedler, P.; Lopes, C.; Alves, E.; Barradas, N.P.; Haueisen, J.; Machado, A.V.; Fonseca, C.; Vaz, F. Ag:TiN-coated polyurethane for dry biopotential electrodes: From polymer plasma interface activation to the first EEG measurements. Plasma Process. Polym. 2016, 13, 341-354. [CrossRef]

43. Barradas, N.P.; Jeynes, C. Advanced physics and algorithms in the IBA DataFurnace. Nucl. Instrum. Methods Phys. Res. Sect. B 2008, 266, 1875-1879. [CrossRef]

44. Liu, Y.; Zhang, J.; Zhang, W.; Liang, W.; Yu, B.; Xue, J. Effects of annealing temperature on the properties of copper films prepared by magnetron sputtering. J. Wuhan Univ. Technol. Mater. Sci. Ed. 2015, 30, 92-96. [CrossRef]

45. Pierson, J.F.; Wiederkehr, D.; Billard, A. Reactive magnetron sputtering of copper, silver, and gold. Thin Solid Films 2005, 478, 196-205. [CrossRef]

46. Figueiredo, V.; Elangovan, E.; Gonçalves, G.; Barquinha, P.; Pereira, L.; Franco, N.; Alves, E.; Martins, R.; Fortunato, E. Effect of post-annealing on the properties of copper oxide thin films obtained from the oxidation of evaporated metallic copper. Appl. Surf. Sci. 2008, 254, 3949-3954. [CrossRef]

47. Borensztein, Y.; Delannoy, L.; Djedidi, A.; Barrera, R.G.; Louis, C. Monitoring of the plasmon resonance of gold nanoparticles in $\mathrm{Au} / \mathrm{TiO}_{2}$ catalyst under oxidative and reducing atmospheres. J. Phys. Chem. C 2010, 114, 9008-9021. [CrossRef]

(C) 2019 by the authors. Licensee MDPI, Basel, Switzerland. This article is an open access article distributed under the terms and conditions of the Creative Commons Attribution (CC BY) license (http://creativecommons.org/licenses/by/4.0/). 

Article

\title{
Nickel Film Deposition with Varying RF Power for the Reduction of Contact Resistance in NiSi
}

\author{
Sunil Babu Eadi ${ }^{1}$, Hyeong-Sub Song ${ }^{1}$, Hyun-Dong Song ${ }^{1}$, Jungwoo Oh ${ }^{2}$ and Hi-Deok Lee ${ }^{1, *}$ \\ 1 Department of Electronics Engineering, Chungnam National University, Daejeon 34134, Korea; \\ sunil@cnu.ac.kr (S.B.E.); hss2310@cnu.ac.kr (H.-S.S.); hd.song@cnu.ac.kr (H.-D.S.) \\ 2 School of Integrated Technology, Yonsei Institute of Convergence Technology, Yonsei University, \\ Incheon 21983, Korea; jungwoo.oh@yonsei.ac.kr \\ * Correspondence: hdlee@cnu.ac.kr; Tel.: +82-042-821-7702
}

Received: 10 May 2019; Accepted: 27 May 2019; Published: 28 May 2019

\begin{abstract}
In this study, the effect of radio frequency (RF) power on nickel (Ni) film deposition was studied to investigate the applications of lowering the contact resistance in the NiSi/Si junction. The RF powers of 100, 150, and $200 \mathrm{~W}$ were used for the deposition of the $\mathrm{Ni}$ film on an $\mathrm{n} / \mathrm{p}$ silicon substrate. RMS roughnesses of $1.354,1.174$ and $1.338 \mathrm{~nm}$ were obtained at 100,150 , and $200 \mathrm{~W}$, respectively. A circular transmission line model (CTLM) pattern was used to obtain the contact resistance for three different RF-power-deposited films. The lowest contact resistivity of $5.84 \times 10^{-5} \Omega$ - $\mathrm{cm}^{2}$ was obtained for the NiSi/n-Si substrate for Ni film deposited at $150 \mathrm{~W}$ RF power.
\end{abstract}

Keywords: deposition rate; contact resistance; nickel silicide; radio frequency

\section{Introduction}

Nickel silicide (NiSi) is a promising metal silicide material for the fabricating source/drain (S/D) contacts in electronic devices; the downscaling of a device leads to an uncontrollable increase in the contact resistance in the $\mathrm{S} / \mathrm{D}$ and gate electrodes [1-3]. NiSi, by virtue of its characteristic properties such as its low-temperature processing, low silicon consumption, and low resistivity phase compared to other metal silicides, has been studied intensively by various research groups. Ramly et al. reported a study on the controlled diffusion of $\mathrm{Ni}$ in the formation of NiSi with different $\mathrm{Ni}$ thicknesses for the application of a supercapacitor electrode [4]. Vijselaar et al. studied the effect of a NiSi interlayer on Si substrates for the fabrication of a photoelectrode for photocatalytic properties [5]. Marshall et al. reported the NiSi as a passivated tunneling contact for application in high-efficiency solar cells [6]. However, obtaining a low-resistivity NiSi phase still remains a critical issue for high-efficiency electronic devices. In this context, obtaining low-resistance NiSi is essential for high-performance devices. Previous reports have shown that, by controlling the Ni diffusion through $\mathrm{Si}$, the NiSi phase can be selectively obtained. Kousseifi et al. used a Pt intermixed layer to control the NiSi phase [7]. Jung et al. used an ultraviolet laser to obtain NiSi through a photo-thermal process [8]. Fouet et al. studied the silicide formation using different $\mathrm{Ni}$ film thicknesses to control NiSi formation [9]. Tous et al. obtained the direct formation of the NiSi phase using the excimer laser annealing (ELA) process [10]. Different methods have been reported for growing NiSi films for various applications. Kwang et al. studied the interfacial properties of NiSi films deposited by using atomic layer deposition [11]. Mahdi et al. reported well-aligned $\mathrm{NiSi} / \mathrm{SiC}$ core-shell nanowire growth by hot-wire chemical vapor deposition to enhance the electrical properties of NiSi [12]. Koichi et al. introduced a cyclic deposition process using molecular beam epitaxy (MBE) to grow NiSi for low-resistance films [13]. Azimirad et al. studied the thermal stability of NiSi film by a co-sputtering process and reported the thermal stability of NiSi to be improved by using a platinum interlayer structure on the Si substrate [14]. 
Therefore, greater understanding of the formation and control of NiSi is needed. To achieve this, the quality and stability of the Ni film are also very important. Ni films can be deposited by various methods such as chemical vapor deposition (CVD), atomic layer deposition (ALD), sputtering, MBE, thermal evaporation, pulsed laser deposition, electroplating [15-21], etc. Among these techniques, vapor deposition through conventional sputtering still remains the most widely used process for metal film deposition. However, to date, the effect of RF power on the contact resistance of the NiSi/Si junctions has not been reported.

In this study, Ni films were deposited with different RF powers, and the influence of the RF power on its surface and structural properties was investigated. Finally, NiSi was obtained by the rapid temperature annealing (RTA) of the different Ni films, and the contact resistance was measured using the circular transmission line model (CTLM) procedure.

\section{Materials and Methods}

\subsection{Ni Films Deposition}

The Ni film samples were deposited by using an RF sputtering process on $\mathrm{Si}(100)$ substrates. Initially, the substrates were cleaned in dilute HF solution for $150 \mathrm{~s}$ and subsequently rinsed in de-ionized water and dried using nitrogen purging. Then, the samples were inserted into the sputtering chamber until the base pressure of the sputtering chamber reached $5.0 \times 10^{-7}$ Torr. After that, an argon flow of $1.8 \mathrm{sccm}$ flowed into the chamber, and the chamber pressure was maintained at $2.5 \mathrm{mTorr}$ using a pressure gauge. The Ni film was deposited at three different RF powers of 100, 150, and $200 \mathrm{~W}$ and the deposition time was fixed to $20 \mathrm{~min}$.

\subsection{Nickel Silicide Fabrication and Contact Resistance Measurement}

Two types of Si substrates were used to measure the contact resistance of NiSi. Arsenic (As) and boron fluoride $\left(\mathrm{BF}_{2}\right)$ were used as $\mathrm{n}$ and $\mathrm{p}$ dopants, by ion implantation with a dose of $5 \times 10^{15} \mathrm{~cm}^{-2}$ at $50 \mathrm{keV}$ to obtain $\mathrm{n} / \mathrm{p}$-Si substrates, respectively. The in-situ deposition of nickel followed by titanium nitride $\mathrm{Ni} / \mathrm{TiN}[15 / 10 \mathrm{~nm}]$ was performed on $\mathrm{n} / \mathrm{p}$-Si substrates at optimized conditions of an Ar flow rate $1.8 \mathrm{sccm}$ and a chamber pressure of $2.5 \mathrm{mTorr}$ and different RF powers of 100, 150 and $200 \mathrm{~W}$, followed by the RTA process at $400{ }^{\circ} \mathrm{C}$ for $30 \mathrm{~s}$ to obtain NiSi. The TiN film was used as a capping layer to prevent the oxidation of Ni during thermal annealing. The CTLM pattern was fabricated on the substrates to measure the contact resistance. Figure 1 shows the experimental flow chart.
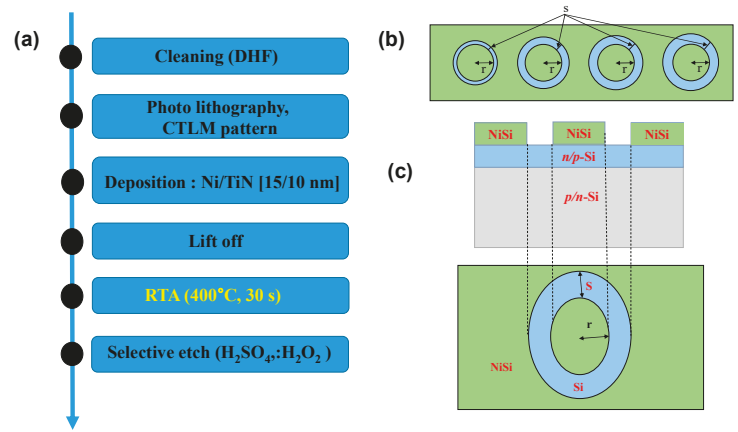

Figure 1. (a) Fabrication flow chart of nickel silicide (NiSi) formations and (b,c) circular transmission line model (CTLM) pattern. $r$ is the radius of the inner circle, and $s$ the radius difference between the inner and outer circle (gap space) and the schematic diagram of NiSi on the Si substrate.

The structural and surface morphologies were characterized using an X-ray diffractometer (XRD, D/MAX 2500PC, Rigaku, Japan) with CuK $\alpha$ radiation, atomic force microscopy (AFM, MAF20 VEECO, 
New York, NY, USA), and field-emission scanning electron microscopy (FESEM, Hitachi, S-4800, Tokyo, Japan). The contact resistance was measured using a Kelvin four-point probe (Alessi REL-6100 Cascade Microtech, Beaverton, OR, USA).

\section{Results and Discussion}

The influence of the RF sputtering power was investigated, keeping all other parameters, such as gas pressure and Ar flow, fixed at $2.5 \mathrm{mTorr}$ and $1.8 \mathrm{sccm}$, respectively. Figure 2 shows the plot of thickness and resistivity as a function of the sputtering power. The plot result shows that by increasing the RF power, the rate of film deposition increases while the resistivity of the film gradually decreases. The average lowest and highest thicknesses of the deposited Ni films obtained were 61.3 and $100 \mathrm{~nm}$ at 100 and $200 \mathrm{~W}$, respectively. The decrease in the resistivity with an increase in the RF power could be due to the decrease in the Ni film crystallite size and surface uniformity. The lowest resistivity of $1.69 \times 10^{-5} \Omega$-cm was obtained for the $150 \mathrm{~W}$-deposited film. It is possible that as the RF power increased, more Ar ions accelerated to hit the Ni target under high power, resulting in more $\mathrm{Ni}$ particles being ejected from the target with increased kinetic energy and velocity, resulting in a faster deposition rate. The high sputtering power improved the crystallization of the Ni films and aided in the formation of the Ni films with highly dense microstructures, which, as a result, led to a reduction in the resistivity $[22,23]$.

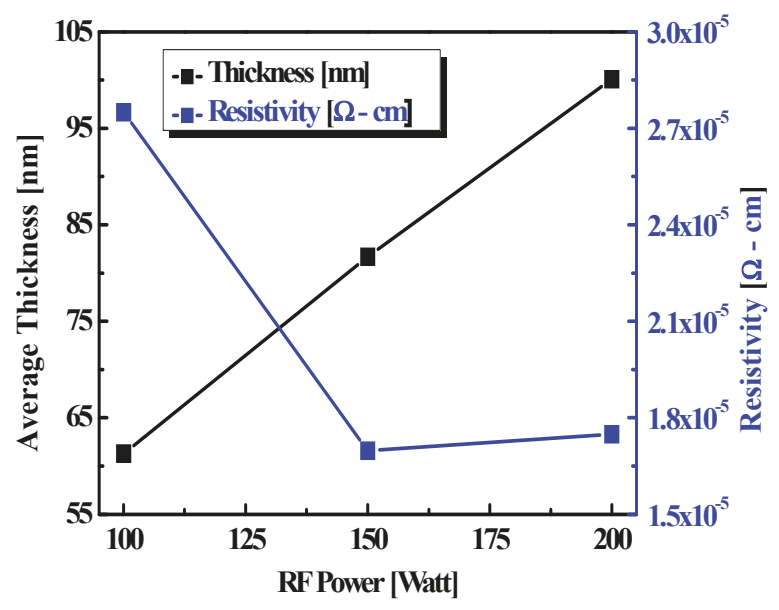

Figure 2. The plot of the Ni films' average thickness and resistivity versus RF power.

To check the surface morphology of the Ni films deposited at various RF powers, FESEM images were taken and are shown in Figure 3. The cross-sectional and surface morphology of the Ni film deposited at $100 \mathrm{~W}$ is shown in Figure 3a-1,a-2. The average film thickness of the Ni film was $61.3 \mathrm{~nm}$ and the surface shows uniform grain disturbance. However, we notice small cracks on the surface of the Ni film. Further, with an increase in the RF power, the Ni film thickness increased. The average thicknesses of the Ni films were 81.7 and $100 \mathrm{~nm}$ for RF powers of 150 and $200 \mathrm{~W}$, respectively, as shown in Figure 3b-2,c-2. The surface morphology of the films shows a bigger crystallite size compared to the $100 \mathrm{~W}$ Ni film. 


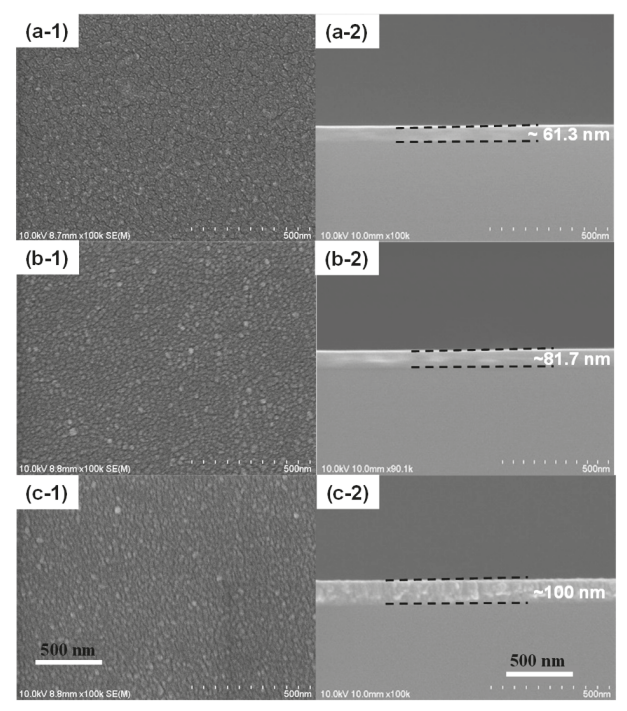

Figure 3. FESEM surface and cross-sectional images of Ni films deposited at different RF powers: $100 \mathrm{~W}(\mathbf{a}-\mathbf{1}, \mathbf{a}-\mathbf{2}), 150 \mathrm{~W}(\mathbf{b}-\mathbf{1}, \mathbf{b}-\mathbf{2})$ and $200 \mathrm{~W}(\mathbf{c}-\mathbf{1}, \mathbf{c}-\mathbf{2})$. The same scale is used for all images $(500 \mathrm{~nm})$.

The structural properties of the films deposited at various magnitudes of RF power are shown in Figure 4 . The main peaks at $44.75^{\circ}, 54.88^{\circ}, 76.41^{\circ}$ are indexed to Ni (111), Ni (200) and Ni (220), which are attributed to Ni's face-centered cubic (FCC) structure [24]. This indicates that the Ni films were in a well-defined crystalline state during deposition. The average crystallite size of the film was determined from the FWHM of the (111) diffraction peaks using Scherrer's equation [25],

$$
D_{\mathrm{p}}=\frac{\mathrm{K} \lambda}{\beta \cos \theta}
$$

where $D_{\mathrm{p}}$ is the average crystallite size, $K=$ Scherrer constant (0.94), $\lambda$ is the x-ray wavelength $(\lambda=1.54178 \AA$ ), $\beta$ is the full width half maximum (FWHM) of the (111) peak, and $\theta$ is the Bragg angle. It was found that the crystallite size varied between $13.59,16.24$, and $14.91 \mathrm{~nm}$ as the RF power changed from 100-200 W. According to the calculations, the crystallite size gradually increased with an increase in the RF power until $150 \mathrm{~W}$, which offered the highest crystallite size, and then gradually decreased. It could be that, with an increase in RF power higher than $150 \mathrm{~W}$, the crystalline quality of film decreases, as evident from the decrease in the relative Ni (111) peak intensity at the higher RF power of $200 \mathrm{~W}$.

To analyze the surface roughness of the $\mathrm{Ni}$ films deposited at different RF powers, AFM measurements were obtained. Figure 5 shows the AFM images at different RF powers. The RMS values of the Ni films obtained were 1.354, 1.174, and $1.338 \mathrm{~nm}$. It was noted that the Ni film surface roughness decreases at $150 \mathrm{~W}$ and gradually increases at $200 \mathrm{~W}$, indicating that the Ni films deposited at higher RF powers show lower film quality. It is observed that the RF power has an influence on the surface structure of Ni films. The Ni films grown at $150 \mathrm{~W}$ RF power have a comparatively low kinetic energy of sputtered particles compared to $200 \mathrm{~W}$ RF power, which leads to a relatively more random orientation and various sizes of grain growth, which lead to a rough surface. 


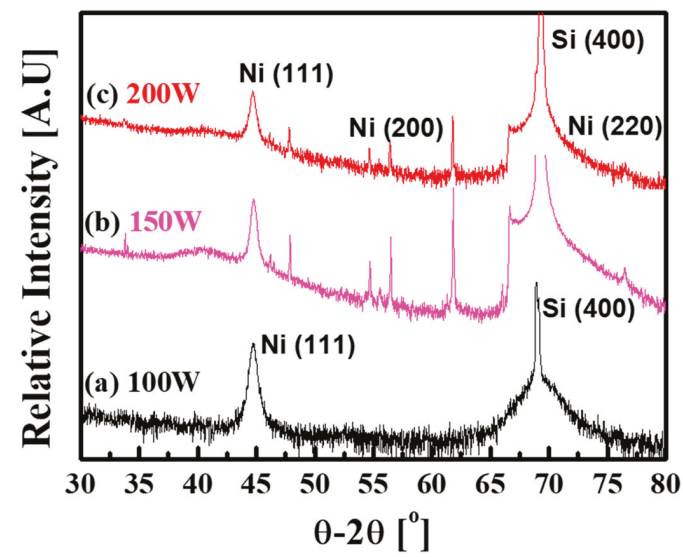

Figure 4. X-Ray diffraction plots of Ni films deposited at different RF powers.

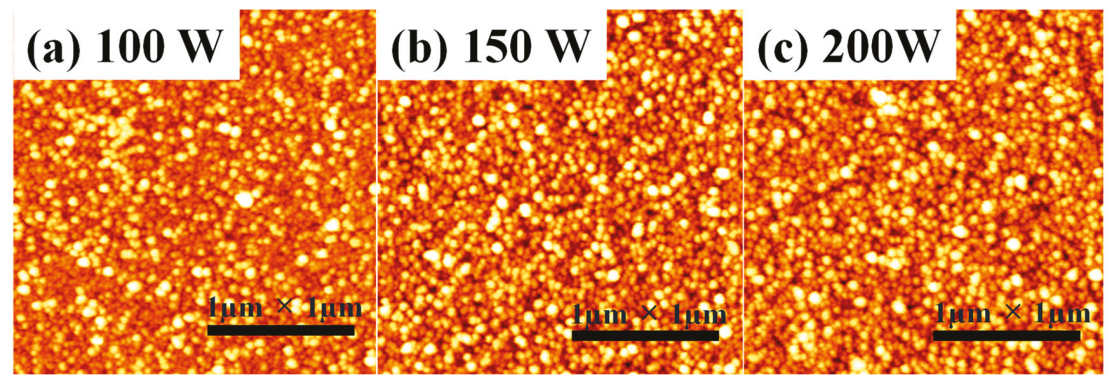

Figure 5. The AFM images Ni films deposited at various RF powers: (a) $100 \mathrm{~W}$, (b) $150 \mathrm{~W}$ and (c) $200 \mathrm{~W}$.

To investigate the effect of RF power on the contact resistance, NiSi films were formed with three different RF powers: 100, 150, and $200 \mathrm{~W}$. An Ni film of $15 \mathrm{~nm}$ was deposited using different sputtering powers on the Si substrates. Prior to deposition, the deposition rates were calculated for all three RF powers, and the corresponding sheet resistances were measured. The deposition rates were calculated by depositing Ni for different growth times of 11-14 min, and plots of thickness versus growth time and deposition rate were obtained by a linear fit. The deposition rates were found to be 4.6, 5.1, and $7.1 \mathrm{~nm} / \mathrm{min}$ at 100,150 , and $200 \mathrm{~W}$, respectively.

In the next section, the three RF conditions are referred to as RF-1, RF-2, and RF-3. Initially, to investigate the lowest sheet resistance of the NiSi phase, Ni films deposited on Si substrates were annealed to form NiSi. The RTA process was performed on the samples with varying temperatures from $300-700{ }^{\circ} \mathrm{C}$ for $30 \mathrm{~s}$ in a $\mathrm{N}_{2}$ atmosphere. Figure 6 shows the plot of sheet resistance $\left(R_{\mathrm{sh}}\right)$ verse RTA temperature; it can be observed that Rsh decreases with increasing temperature from $300{ }^{\circ} \mathrm{C}$ until $450{ }^{\circ} \mathrm{C}$, and with further increase in temperature, the Rsh increases drastically. It is well known that at a lower temperature $\left(300-400{ }^{\circ} \mathrm{C}\right)$, the NiSi phase predominantly exists, and with an increase in the temperature, Rsh increases. The lowest Rsh values of 6.04 and $6.46 \Omega / s q$. were obtained for RF-2 on $p$ and n-Si substrates, respectively. Above the RTA temperature of $550{ }^{\circ} \mathrm{C}$, the sheet resistance increases drastically due to the predominant formation of $\mathrm{NiSi}_{2}$, which is the high-resistance phase. Zhao et al. have reported that above $500{ }^{\circ} \mathrm{C}$, the agglomeration of $\mathrm{NiSi}$ increases and irregular nucleation of $\mathrm{NiSi}_{2}$ starts, which leads to an increase in resistance [26]. 


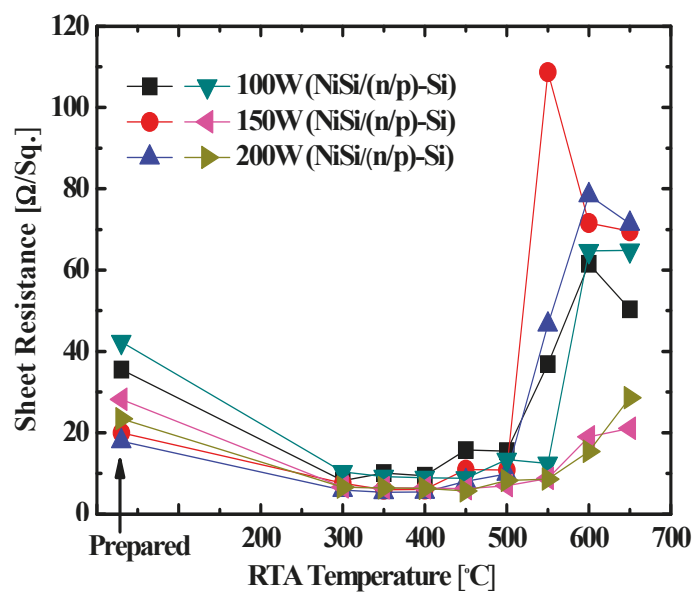

Figure 6. The plot of sheet resistance verses RTA temperature plot of NiSi on n/p-Si formed with different RF powers.

The specific contact resistance $\rho_{\mathrm{C}}$ between the Si substrate and NiSi for the three different $\mathrm{Ni}$ films were extracted using a 4-wire Kelvin resistance measurement, and $\rho_{\mathrm{C}}$ was determined using the following equations [27]:

$$
\begin{gathered}
R_{\mathrm{T}}=\frac{R_{\mathrm{sh}}}{2 \pi \mathrm{r}}\left(s+2 L_{\mathrm{T}}\right) C \\
C=\frac{r}{s} \ln \left(1+\frac{s}{r}\right) \\
\rho_{\mathrm{C}}=R_{\mathrm{sh}} L_{\mathrm{T}}^{2}
\end{gathered}
$$

where $R_{\mathrm{sh}}$ is the sheet resistance, $\mathrm{C}$ is the correction factor, $L_{\mathrm{T}}$ is the effective transfer length, $r$ is the radius of the inner circle, which was fixed at $80 \mathrm{~mm}$, and $s$ is the gap space, which was split as $8,12,16,20,24,32,40$, and $48 \mathrm{~mm}$. $R_{\mathrm{sh}}$ and $L_{\mathrm{T}}$ can be determined via a linear fit of $R_{\mathrm{T}}$ at the different gap space values. Figure 7 shows the plot of total resistance measured as a function of the gap space between the inner and outer rings of the NiSi layers for RF-1, RF-2, and RF-3. The lower total contact resistivities of $5.84 \times 10^{-5} \Omega-\mathrm{cm}^{2}$ for n-Si and $6.58 \times 10^{-5} \Omega-\mathrm{cm}^{2}$ for $\mathrm{p}$-Si are obtained for the RF-2 film, respectively, and the reduction in contact resistance is lower than the Ni Ohmic contacts previously reported [28]. Thus, by controlling the film deposition using RF power, the quality of the NiSi formation can be controlled. A similar conclusion was reported by Gordillo et al. in their work done on the effect of RF power on electrical properties of Mo films. They concluded from their study that a decrease in the resistivity of Mo films was caused by changing the RF power [29]. In our case, different RF powers control the grain size and surface roughness of the Ni films and show different NiSi electrical characteristics. 


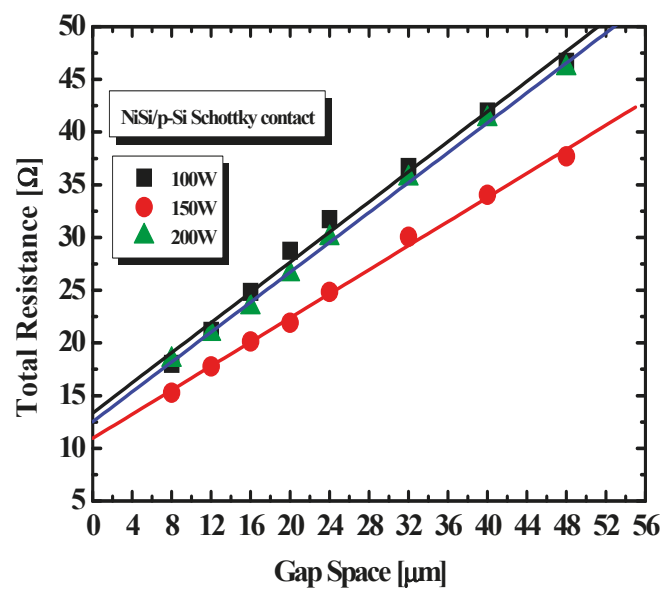

Figure 7. The plot of the total resistance versus gap space of the CTLM pattern for the extraction of specific contact resistivity's with different RF powers.

Table 1 shows the measured values of the contact resistivity and transfer length of the NiSi on $\mathrm{n} / \mathrm{p}$-Si substrates. Thus, from the results, it could be that Ni films deposited with an RF power of $150 \mathrm{~W}$ showed lower contact resistance in comparison to the Ni films at 100 and $200 \mathrm{~W}$ power. It is well known that $\mathrm{Ni}$ film and $\mathrm{Si}$ substrate undergo a sequential reaction from $\mathrm{Ni}_{2} \mathrm{Si}$ to $\mathrm{NiSi}$ and finally to $\mathrm{NiSi}_{2}$ with an increase in the RTA temperature [30-32]. In our case, the formation of the sequence of $\mathrm{Ni}$ silicide phases of the three films varied with the Ni deposition rate and RTA temperature. The Ni film deposited at $150 \mathrm{~W}$ could have controlled the NiSi phase with lower resistivity compared to the other two RF powers. These results lead to the conclusion that, by controlling the Ni film deposition rates, it is possible to obtain low-resistivity NiSi for future electronic devices.

Table 1. The values of the contact resistivity and transfer length of the NiSi on n/p-Si substrates.

\begin{tabular}{cccccc}
\hline \multirow{2}{*}{ Sample } & \multicolumn{2}{c}{ NiSi/n-Si } & \multicolumn{2}{c}{ NiSi/p-Si } \\
\cline { 2 - 6 } & RF Power & $\rho_{\mathrm{C}}\left[\Omega-\mathrm{cm}^{2}\right]$ & $L_{\mathrm{T}}[\mu \mathrm{m}]$ & $\rho_{\mathrm{C}}\left[\Omega-\mathrm{cm}^{2}\right]$ & $L_{\mathrm{T}}[\mu \mathrm{m}]$ \\
\hline $\mathrm{Ni} / \mathrm{TiN}$ & $100 \mathrm{~W}$ & $8.08 \times 10^{-5}$ & 8.128 & $7.82 \times 10^{-5}$ & 4.663 \\
\cline { 2 - 6 }$[15 / 10$ & $150 \mathrm{~W}$ & $5.84 \times 10^{-5}$ & 7.493 & $6.58 \times 10^{-5}$ & 4.787 \\
\cline { 2 - 6 } $\mathrm{nm}]$ & $200 \mathrm{~W}$ & $1.18 \times 10^{-4}$ & 10.29 & $6.94 \times 10^{-5}$ & 4.411 \\
\hline
\end{tabular}

\section{Conclusions}

In this study, Ni films formed at three different RF powers of 100, 150, and $200 \mathrm{~W}$ were investigated for low-resistivity NiSi contacts. The result analysis shows a decrease in the resistivity of Ni films with an increase in RF power. The XRD and AFM data on the Ni films clearly support the change in the resistivity with RF power change. Further, low contact resistance NiSi films were formed by annealing the Ni films, and their contact resistances were measured. The total resistance of $5.84 \times 10^{-5} \Omega-\mathrm{cm}^{2}$ was obtained for $150 \mathrm{~W}$ RF power. The results clearly show that the NiSi formed at $150 \mathrm{~W}$ showed lower resistance than NiSi formed at 100 and $200 \mathrm{~W}$. This study is meaningful in that a thin Ni film with a low resistivity was formed by varying the RF power in sputtering, and the formed NiSi phase showed a low contact resistance.

Author Contributions: Conceptualization and Supervision, H.-D.L.; Methodology and Writing-Original Draft Preparation, S.B.E., H.-S.S.; Writing-Review and Editing, H.-D.S., H.-D.L. and J.W.O.; Funding Acquisition, H.-D.L. 
Funding: This research was funded by the MOTIE (Ministry of Trade, Industry \& Energy (10048536) and the KSRC (Korea Semiconductor Research Consortium) support program for the development of future semiconductor devices. This research was also supported by the Ministry of Trade, Industry and Energy (MOTIE) (10067808) and the Korea Semiconductor Research Consortium (KSRC) support program for the development of future semiconductor devices.

Conflicts of Interest: The authors declare no conflict of interest.

\section{References}

1. Rabab, R.B.; Amir, N.H.; Arwa, T.K.; Abdurrahman, G.; Muhammad, M.H. Impact of nickel silicide rear metallization on the series resistance of crystalline silicon solar cells. Energy Technol. 2018, 6, 1627-1632.

2. Geenen, F.A.; Van Stiphout, K.; Nanakoudis, A.; Bals, S.; Vantomme, A.; Jordan-Sweet, J.; Lavoie, C.; Detavernier, C. Controlling the formation and stability of ultra-thin nickel silicides-An alloying strategy for preventing agglomeration. J. Appl. Phys. 2018, 123, 075303. [CrossRef]

3. Wolansky, D.; Grabolla, T.; Lenke, T.; Schulze, S.; Zaumseil, P. Impact of nickel silicide on SiGe BiCMOS devices. Semicond. Sci. Technol. 2018, 33, 124003. [CrossRef]

4. Ramly, M.M.; Omar, F.S.; Rohaizad, A.; Aspanut, Z.; Rahman, S.A.; Goh, B.T. Solid-phase diffusion controlled growth of nickel silicide nanowires for supercapacitor electrode. Appl. Surf. Sci. 2018, 456, 515-525. [CrossRef]

5. Vijselaar, W.; Tiggelaar, R.M.; Gardeniers, H.J.; Huskens, J. Efficient and stable silicon microwire photocathodes with a nickel silicide interlayer for operation in strongly alkaline solutions. ACS Energy Lett. 2018, 3, 1086-1092. [CrossRef] [PubMed]

6. Marshall, A.; Florent, K.; Tapriya, A.; Lee, B.G.; Kurinec, S.K.; Young, D.L. Nickel silicide metallization for passivated tunneling contacts for silicon solar cells. In Proceedings of the IEEE 43rd Photovoltaic Specialists Conference (PVSC), Portland, OR, USA, 5-10 June 2016; pp. 2479-2482.

7. El Kousseifi, M.; Hoummada, K.; Bertoglio, M.; Mangelinck, D. Selection of the first Ni silicide phase by controlling the Pt incorporation in the intermixed layer. Acta Mater. 2016, 106, 193-198. [CrossRef]

8. Jung, S.M.; Kim, J.H.; Park, C.J.; Shin, M.W. Nickel mono-silicide formation using a photo-thermal process assisted by ultra-violet laser. Mater. Sci. Semicond. Process. 2018, 75, 263-268. [CrossRef]

9. Richard, M.-I.; Mangelinck, D.; Guichet, C.; Thomas, O.; Texier, M.; Fouet, J.; Boudet, N.; Portavoce, A. Silicide formation during reaction between Ni ultra-thin films and Si (001) substrates. Mater. Lett. 2013, 116, 139-142.

10. Tous, L.; Lerat, J.-F.; Emeraud, T.; Negru, R.; Huet, K.; Uruena, A.; Aleman, M.; Russell, R.; John, J.; Poortmans, J.; et al. Nickel silicide formation using excimer laser annealing. Energy Procedia 2012, 27, 503-509. [CrossRef]

11. Lee, K.M.; Kim, C.Y.; Choi, C.K.; Yun, S.W.; Ha, J.B.; Lee, J.H.; Lee, J.Y. Interface properties of nickel-silicide films deposited by using plasma-assisted atomic layer deposition. J. Korean Phys. Soc. 2009, 55, 1153-1157. [CrossRef]

12. Alizadeh, M.; Hamzan, N.B.; Ooi, P.C.; Bin Omar, M.F.; Dee, C.F.; Goh, B.T. Solid-state limited nucleation of $\mathrm{NiSi} / \mathrm{SiC}$ core-shell nanowires by hot-wire chemical vapor deposition. Materials 2019, 12, 674. [CrossRef]

13. Terashima, K.; Miura, Y.; Ikarashi, N.; Oshida, M.; Manabe, K.; Yoshihara, T.; Tanaka, M.; Wakabayashi, H. Formation of Nickel Self-Aligned Silicide by Using Cyclic Deposition Method. In Proceedings of the 2004 International Conference on Solid State Devices and Materials, Tokyo, Japan, 14-17 September 2004; pp. 182-183.

14. Azimirad, R.; Kargarian, M.; Akhavan, O.; Moshfegh, A.Z. Improved thermal stability of NiSi nanolayer in Ni-Si Co-sputtered structure. Int. J. Nanosci. Nanotechnol. 2011, 7, 14-20.

15. Guo, Q.; Guo, Z.; Shi, J.; Sang, L.; Gao, B.; Chen, Q.; Liu, Z.; Wang, X. Fabrication of nickel and nickel carbide thin films by pulsed chemical vapor deposition. MRS Commun. 2018, 8, 88-94. [CrossRef]

16. Han, W.S.; Kim, S.; Hwang, J.; Park, J.-M.; Koh, W.; Lee, W.-J. Plasma-enhanced atomic layer deposition of nickel thin film using is (1,4-diisopropyl-1,4-diazabutadiene) nickel. J. Vac. Sci. Technol. A Vacuum Surf. Film 2017, 36, 01A119.

17. Widodo, S. Characterization of Thin Film Nickel (Ni) Deposition by Sputtering Method. Int. J. Innov. Sci. Technol. 2015, 2, 380-385. 
18. Nishiyama, T.; Tanaka, T.; Shikada, K.; Ohtake, M.; Kirino, F.; Futamoto, M. Growth of Ni Thin Films on $\mathrm{Al}_{2} \mathrm{O}_{3}$ Single-Crystal Substrates. Jpn. J. Appl. Phys. 2009, 48, 13003. [CrossRef]

19. Valladares, L.D.L.S.; Ionescu, A.; Holmes, S.; Barnes, C.H.W.; Dominguez, A.B.; Quispe, O.A.; Gonzalez, J.C.; Milana, S.; Barbone, M.; Ferrari, A.C.; et al. Characterization of Ni thin films following thermal oxidation in air. J. Vac. Sci. Technol. B 2014, 32, 51808. [CrossRef]

20. Kassem, W.; Roumie, M.; Tabbal, M. Pulsed Laser Deposition of Tungsten Thin Films on Graphite. Adv. Mater. 2011, 324, 77-80. [CrossRef]

21. Li, J.-D.; Zhang, P.; Wu, Y.-H.; Liu, Y.-S.; Xuan, M. Uniformity study of nickel thin-film microstructure deposited by electroplating. Microsyst. Technol. 2009, 15, 505-510. [CrossRef]

22. Peri, B.; Borah, B.; Dash, R.K. Effect of RF power and gas flow ratio on the growth and morphology of the PECVD SiC thin film s for MEMS applications. Mater. Sci. 2015, 38, 1105-1112. [CrossRef]

23. Posadowski, W.; Wiatrowski, A.; Kapka, G. Effect of pulsed magnetron sputtering process for the deposition of thin layers of nickel and nickel oxide. Mater. Sci. 2018, 36, 69-74. [CrossRef]

24. Vergara, J.; Madurga, V. Structure and magnetic properties of Ni films obtained by pulsed laser ablation deposition. J. Mater. 2002, 17, 2099-2104. [CrossRef]

25. D'Agostino, A.T. Determination of thin metal film thickness by X-ray diffractometry using the Scherrer equation, atomic absorption analysis and transmission/reflection visible spectroscopy. Anal. Chim. Acta 1992, 262, 269-275. [CrossRef]

26. Zhao, F.F.; Zheng, J.Z.; Shen, Z.X.; Osipowicz, T.; Gao, W.Z.; Chan, L.H. Thermal stability study of NiSi and $\mathrm{NiSi}_{2}$ thin films. Microelectron. Eng. 2004, 71, 104-111. [CrossRef]

27. Schroder, D.K. Semiconductor Material and Device Characterization, 3rd ed.; John Wiley \& Sons, Inc.: Hoboken, NJ, USA, 2006; p. 144. ISBN 9780471739067.

28. Kuchuk, A.V.; Borowicz, P.; Wzorek, M.; Borysiewicz, M.; Ratajczak, R.; Golaszewska, K.; Kaminska, E.; Kladko, V.; Piotrowska, A. Ni-based ohmic contacts to n-type 4H-SiC: The formation mechanism and thermal stability. Adv. Condens. Matter Phys. 2016, 2016, 26. [CrossRef]

29. Gordillo, G.; Mesa, F.; Calderón, C. Electrical and morphological properties of low resistivity Mo thin Films prepared by magnetron sputtering. Braz. J. Phys. 2006, 36, 982-985. [CrossRef]

30. Zhang, S.-L.; Smith, U. Self-aligned silicides for Ohmic contacts in complementary metal-oxide-semiconductor technology: $\mathrm{TiSi}_{2}, \mathrm{CoSi}_{2}$, and NiSi. J. Vac. Sci. Technol. A Vacuum Surf. Film 2004, 22, 1361-1370. [CrossRef]

31. Chen, X.; Zhang, B.; Li, C.; Shao, Z.; Su, D.; Williams, C.T.; Liang, C. Structural and electrochemical properties of nanostructured nickel silicides by reduction and silicification of high-surface-area nickel oxide. Mater. Res. Bull. 2012, 47, 867-877. [CrossRef]

32. Lauwers, A.; Kitt, J.A.; Van Dal, M.J.H.; Chamirian, O.; Pawlak, M.A.; De Potter, M.; Lindsay, R.; Raymakers, T.; Pages, X.; Mebarki, B. Ni based suicides for $45 \mathrm{~nm} \mathrm{CMOS}$ and beyond. Mater. Sci. Eng. B Solid-State Mater. Adv. Technol. 2004, 114-115, 29-41. [CrossRef]

(C) 2019 by the authors. Licensee MDPI, Basel, Switzerland. This article is an open access article distributed under the terms and conditions of the Creative Commons Attribution (CC BY) license (http://creativecommons.org/licenses/by/4.0/). 

Article

\title{
Experimental and Modeling Study of the Fabrication of Mg Nano-Sculpted Films by Magnetron Sputtering Combined with Glancing Angle Deposition
}

\author{
Hui Liang ${ }^{1,2}$, Xi Geng ${ }^{1}$, Wenjiang Li ${ }^{1,3, *}$, Adriano Panepinto ${ }^{2}$, Damien Thiry ${ }^{2}$, \\ Minfang Chen ${ }^{1,3, *}$ and Rony Snyders ${ }^{2,4, *}$ \\ 1 School of Materials Science and Engineering, Tianjin University of Technology, Tianjin 300384, China; \\ hui.liang@umons.ac.be (H.L.); xigeng0220@126.com (X.G.) \\ 2 Chimie des Interactions Plasma-Surface, University of Mons, 20 Place du Parc, B 7000 Mons, Belgium; \\ adriano.panepinto@umons.ac.be (A.P.); Damien.THIRY@umons.ac.be (D.T.) \\ 3 Key Laboratory of Display Materials and Photoelectric Device (Ministry of Education), Tianjin 300384, China \\ 4 Materia Nova Research Center, 1 Avenue Nicolas Copernic, B 7000 Mons, Belgium \\ * Correspondence: liwj@tjut.edu.cn (W.L.); jgj@tjut.edu.cn (M.C.); rony.snyders@umons.ac.be (R.S.); \\ Tel.: +32-65-554955 (R.S.)
}

Received: 5 May 2019; Accepted: 27 May 2019; Published: 1 June 2019

\begin{abstract}
Today, $\mathrm{Mg}$ is foreseen as one of the most promising materials for hydrogen storage when prepared as nano-objects. In this context, we have studied the fabrication of Mg nano-sculpted thin films by magnetron sputtering deposition in glancing angle configuration. It is demonstrated that the microstructure of the material is controllable by tuning important deposition parameters such as the tilt angle or the deposition pressure which both strongly affect the shadowing effect during deposition. As an example, the angle formed by the column and the substrate and the intercolumnar space varies between $\sim 20^{\circ}$ to $\sim 50^{\circ}$ and $\sim 45$ to $\sim 120 \mathrm{~nm}$, respectively, when increasing the tilt angle from $60^{\circ}$ to $90^{\circ}$. These observations are highlighted by modeling the growth of the material using kinetic Monte Carlo methods which highlights the role of surface diffusion during the synthesis of the coating. This work is a first step towards the development of an air-stable material for hydrogen storage.
\end{abstract}

Keywords: Mg columnar films; glancing angle deposition; magnetron sputtering; kinetic Monte Carlo modeling

\section{Introduction}

With increased worldwide energy consumption that is associated with the global warming problem and the depletion of fossil fuels, renewable energy sources from hydro, solar, and wind sources are increasingly replacing the conventional fuels $[1,2]$. This is the driving force of a real appeal for the development of new solutions in several domains of our society, including the transport industry. Considering the latter, today, several strategies are considered to design the car of the future, and among them, the hydrogen car is one of the most promising ones. Indeed, hydrogen can be produced by various electrochemical and biological methods and has a higher chemical energy as compared with fossil fuels [3-5]. Furthermore, once produced from any energy source, hydrogen generates electricity during fuel cell operations, leaving water vapor as the only exhaust gas, without any other greenhouse gases or harmful emissions [2]. Nevertheless, several issues related to the production, distribution, and storage of hydrogen have to be fixed before using hydrogen as an economically viable fuel for the transport industry [6]. In particular, the hydrogen storage is an important issue related to the low volumetric density of hydrogen. Among the solutions developed to store hydrogen, the utilization of solid-state materials is preferred because of its higher volumetric density (as compared with gaseous 
and liquid solutions) and for safety reasons. Among the solid-state materials that store hydrogen, the hydride materials where hydrogen is chemically bounded (i.e., not only adsorbed) appear to be good candidates [7-9].

Specifically, magnesium-based hydrides, and more specifically elemental magnesium hydride $\left(\mathrm{MgH}_{2}\right)$, are often considered as promising materials for hydrogen storage because magnesium $(\mathrm{Mg})$ is abundant, low cost, has low density, low toxicity and higher hydrogen capacity and reversibility as compared with other hydrides [10,11]. Nevertheless, this material suffers two main drawbacks which are a high desorption temperature and a slow hydrogen sorption kinetic [11]. In addition, $\mathrm{Mg}$ can easily be oxidized by oxygen and hydrogen not easily diffused in bulk Mg.

For many years, these problems have been addressed by the community. A complete review on the topic has recently been published by Sadhasivam et al. [12]. From these works, it appears that the reduction of the size of the $\mathrm{Mg}$ compounds down to the nanoscale strongly improves the thermodynamic properties of the material [13]. Therefore, several routes have been investigated to reduce the size of the $\mathrm{Mg} / \mathrm{MgH}_{2}$ (below $1 \mu \mathrm{m}$ ) particles such as mechanical ball milling in the presence (or not) of catalyst materials leading to significant improvements in term of the sorption kinetic of the material [14]. Nevertheless, if the sorption kinetic is improved by this approach, this is not the case for the thermodynamic parameters [14]. In order to overcome this problem, it has been suggested that a further reduction of the dimension $(<100 \mathrm{~nm})$ of the material could help. This is why efforts have been developed in order to fabricate 1-, 2- or 3D Mg nanoparticles [6,10]. As an example, Barawi et al. $[15,16]$ reported on the synthesis of $\mathrm{Mg}$ films by e-beam evaporation on $\mathrm{SiO}_{2}$ substrates with a thickness ranging from 45 to $900 \mathrm{~nm}$ and demonstrated that it plays a major role in the hydrogen absorption kinetics.

In this context and in view of the material science challenges, plasma techniques appear as an ideal technological platform to synthesize these materials. Indeed, these technologies are known as "green" technologies, since they allow for good control of the material properties and their industrial transfer has been demonstrated in many fields such as the glass industry or microelectronics [17-21]. Among these technologies, magnetron sputtering (MS) is usually used to grow dense thin films of various materials (from metal to polymer coatings) [21-23]. Nevertheless, when used in the glancing angle deposition (GLAD) configuration, it has been demonstrated that nanostructurated coatings for the microstructure can be controlled. As an example, we recently reported on the growth of Ti and $\mathrm{TiO}_{2}$ nanostructurated films by using this approach $[19,20]$.

Therefore, in this work, we aim to study the growth of nano-sculpted $\mathrm{Mg}$ films by combining magnetron sputtering and glancing angle deposition (MSGLAD) in order to better understand the growth mechanism of this material which could ultimately be used in composite material for hydrogen storage application. Our strategy consists of a systematic study of the influence of important deposition parameters namely the tilt angle $(\alpha)$ and the working pressure $\left(P_{\text {Tot }}\right)$ on the microstructure of the synthesized material. These experimental results are compared to computer simulation by Kinetic Monte Carlo (KMC) using the NASCAM code to better understand the growth mechanism of the Mg thin films.

\section{Material and Method}

\subsection{Experimental}

All experiments were carried out in a cylindrical stainless-steel chamber (height: $60 \mathrm{~cm}$, diameter: $42 \mathrm{~cm}$ ), shown in Figure 1. The chamber was evacuated by a turbo-molecular pump (Edwards nEXT400D 160W, Burgess Hill, UK), down to a residual pressure of $10^{-7} \mathrm{~Pa}$. A magnetron cathode was installed at the top of the chamber and the substrate was located at a distance of $80 \mathrm{~mm}$. A 2-inch in diameter and 0.25 -inch thick $\mathrm{Mg}$ target (99.99\% purity) was used. The target was sputtered in DC mode using an Advanced Energy MDK $1.5 \mathrm{~K}$ power supply in argon atmosphere using a flow of 
$12 \mathrm{sccm}$. Conductive silicon wafers (100) were used as substrates and rinsed with ultra-pure water before deposition.

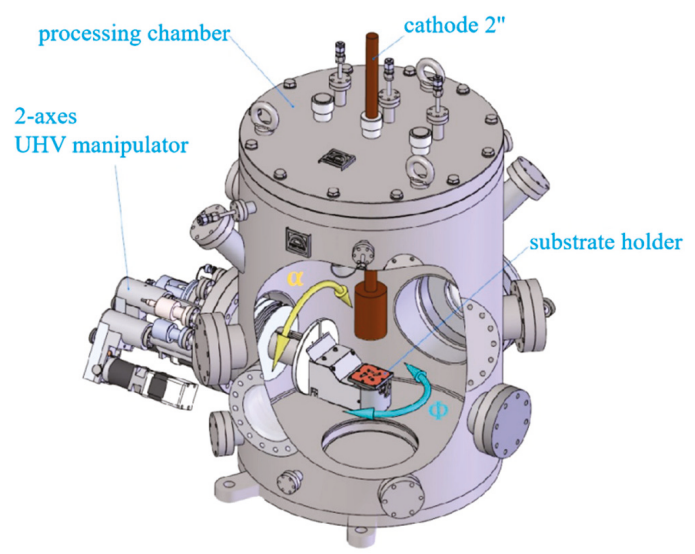

Figure 1. Sketch of the deposition chamber used in this work.

Using the GLAD system, the substrate can be tilted with an angle $\alpha$ and eventually rotated by an angle $(\varphi)$ either step-by-step or with a continuous angular speed in order to generate diverse thin film architectures. In this work, we have only studied the effect of the tilt angle on the architecture of the deposited films with $\alpha=60^{\circ}, 80^{\circ}, 82.5^{\circ}, 85^{\circ}, 87^{\circ}$, and $89^{\circ}$. On the other hand, we also evaluated the influence of the working pressure $\left(P_{\mathrm{Tot}}\right)$ which was varied from 0.13 to $1.3 \mathrm{~Pa}$. For all deposition, the sputtering power was kept constant at $50 \mathrm{~W}$ and the deposition time varied between 10 and $20 \mathrm{~min}$ depending on the deposition conditions in order to reach similar thicknesses for all deposited films.

The morphology of the material was characterized with a field emission gun scanning electron microscope (FEG-SEM, Hitachi SU8020, Ri Li, Japan). In addition, from the SEM images, we extracted the so-called aspect ratio, $\Gamma$, which is defined as the ratio between the inter-columnar space and the column width.

The chemical composition of the films was evaluated by X-ray photoelectron spectroscopy (XPS) on a VERSAPROBE PHI 5000 hemispherical analyzer from Physical Electronics with a base pressure of $<3 \times 10^{-7} \mathrm{~Pa}$. The X-ray photoelectron spectra were collected at the take-off angle of $45^{\circ}$ with respect to the electron energy analyzer, operating in constant analyzer energy (CAE) mode $(23.50 \mathrm{eV})$. The spectra were recorded with the monochromatic $\mathrm{Al} \mathrm{K \alpha}$ radiation $(15 \mathrm{kV}, 25 \mathrm{~W})$ with a highly focused beam size of $100 \mu \mathrm{m}$. The energy resolution was $0.7 \mathrm{eV}$. Eventual surface charging was compensated for by a built-in electron gun and an argon ion neutralizer. For the chemical depth profile, an $\mathrm{Ar}^{+}$ion source was operated at $1 \mu \mathrm{A}$ and $2 \mathrm{kV}$ with a raster area of $2 \mathrm{~mm} \times 2 \mathrm{~mm}$ at an incident angle normal to the sample surface of $54.7^{\circ}$. The XPS spectra were referenced to the $\mathrm{Mg} 2 \mathrm{p}$ peak at $49.5 \mathrm{eV}$ arising from the metallic magnesium component [24]. Atomic compositions were derived from peak areas using photoionization cross sections calculated by Scofield, corrected for the dependence of the escape depth on the kinetic energy of the electrons and corrected for the analyzer transmission function of our spectrometer.

The thickness of the films, as measured by a mechanical profilometer Dektak 150 from Veeco, was kept constant for all films, and their average thickness was about $620 \pm 20 \mathrm{~nm}$. As an example, the deposition rate was $0.32 \mathrm{~nm} / \mathrm{s}$ for a deposition angle of $85^{\circ}$ and a sputtering pressure of $2 \mathrm{mTorr}$.

Finally, the phase constitution of the samples was evaluated by X-ray diffraction (XRD) using a PANalytical Empyrean diffractometer working with $\mathrm{Cu} \mathrm{K}_{\alpha 1}$ radiation $(\lambda=0.1546 \mathrm{~nm})$ in the grazing incidence configuration $\left(\Omega=0.5^{\circ}\right)$. The $\mathrm{X}$-ray source voltage was fixed at $45 \mathrm{kV}$ and the current 
at $40 \mathrm{~mA}$. The grain size $\left(G_{S}\right)$ was calculated from the XRD pattern using the following Scherrer equation [25]:

$$
G_{s}=\frac{K \lambda}{\beta \cos \theta}
$$

where, $K$ is a dimensionless shape factor, $\lambda$ is the $X$-ray wavelength, $\beta$ is the diffraction line broadening at half the maximum intensity (FWHM), and $\theta$ is the Bragg angle.

\subsection{Simulation}

NASCAM is an atomistic deposition simulation code based on the kinetic Monte Carlo (kMC) method. It can be used for the modeling of different processes occurring at the surface such as the growth of films during deposition. The atoms are deposited on the substrate at random positions at an equal time interval which is determined by the deposition rate. Only diffusion or evaporation events can take place between two deposition events. Energy transfer during ballistic collision events is also taken into account. This made it suitable to simulate glancing angle deposition processes [26]. The energy and angular distribution of incident particles were calculated by SRIM [27] and SIMTRA [28]. First, SRIM was used to calculate the energy and the direction of particles which leave the target. The particles were then transported in the gas phase by the SIMTRA code which took into account all the collisions between the sputtered species and the gas molecules. The energy and the angular distribution of the species at the substrate location were derived for each working conditions by the introduction of the experimental parameters, which included the working pressure, the particles' energy which was a function of the power applied at the target, the racetrack sizes, and the target-to-substrate distance. After that, these files were used as input data for NASCAM. Other parameters could be tuned in the input file. To compare the simulation with the experience, we tuned the number of deposited atoms and the substrate size (XYZ). In these conditions, the simulated and the experimental thin film had the same thickness.

The energy and the angular distribution of the species at the substrate location were derived for each working conditions by the introduction of the experimental parameters such as the working pressure, the power applied to the target, the racetrack size, and the target-to-substrate distance, Other parameters could be varied in the NASCAM input file such as the number of deposited atoms $(\mathrm{N})$ and the substrate size (XYZ). In order to compare simulated and experimental thin films, both had the same thickness. For direct comparison of the cross-sectional film morphology, "2D" $(Y=2)$ NASCAM simulations were performed ( $\mathrm{N}=1000$ atoms), whereas, for density and porosity evaluation, "3D" $(\mathrm{Y}=4)$ simulations were performed ( $\mathrm{N}=1.667$ atoms). The deposition rate was fixed at 0.5 monolayer by second $(0.301 \mathrm{~nm} / \mathrm{s})$, which was close to the experimental value $(0.32 \mathrm{~nm} / \mathrm{s})$ at a deposition angle of $85^{\circ}$.

\section{Results and Discussion}

\subsection{Characterization of A Dense Mg Film}

In a first attempt, we have grown a $\mathrm{Mg}$ thin film in conventional geometry $\left(\alpha=0^{\circ}\right)$ in order to evaluate the deposition rate, as well as the chemical composition and the phase constitution of the deposited material. Figure 2 shows the survey XPS spectrum recorded for this film. It reveals the presence of $\mathrm{Mg}, \mathrm{O}$, and $\mathrm{C}$ lines at $49.5 \mathrm{eV}(\mathrm{Mg} 2 \mathrm{p}), 285 \mathrm{eV}(\mathrm{C} 1 \mathrm{~s})$, and $530 \mathrm{eV}(\mathrm{O} 1 \mathrm{~s})$. From the quantification of these signal, $\sim 50$ atom $\%$ of oxygen and 10 atom $\%$ of carbon are observed. These are likely related to surface pollution that appears during the transport of the sample from the chamber to the XPS machine. Particularly, the presence of oxygen while working in non-reactive conditions is related strong reactivity of $\mathrm{Mg}$ towards $\mathrm{O}_{2}\left(\Delta H_{f(\mathrm{MgO})}=-601.8 \mathrm{~kJ} \cdot \mathrm{mol}^{-1}\right)$ [29], which allows for the oxidation of the top surface of the deposited film. In order to clarify the chemistry of the film and to validate that the presence of carbon, as well as the surface oxidation of $\mathrm{Mg}$, are related to surface pollution, depth profiling of the films were performed by using an $\mathrm{Ar}^{+}$gun in the XPS machine before recording the XPS spectra during 2, 4, and $20 \mathrm{~min}$ of erosion. The results are presented in Table 1. Clearly, it appears from this analysis that a few minutes of erosion allows removal of all the initially observed carbon contamination 
as well as reduction of the oxygen content to an $\sim 10 \mathrm{atom} \%$ limit, validating the surface contamination of the as-deposited sample. The presence of the 10 atom\% of oxygen in the bulk of the material is likely explained by the presence of very low quantity adsorbed water or oxygen in the deposition chamber even if the base pressure is good quality $\left(10^{-7}\right.$ Torr). Indeed, because of the already mentioned strong reactivity of $\mathrm{Mg}$ towards $\mathrm{O}_{2}$, a getter effect likely occurs and leads to the partial oxidation of the material similar to the effect already observed for another getter material such as Ti [30].

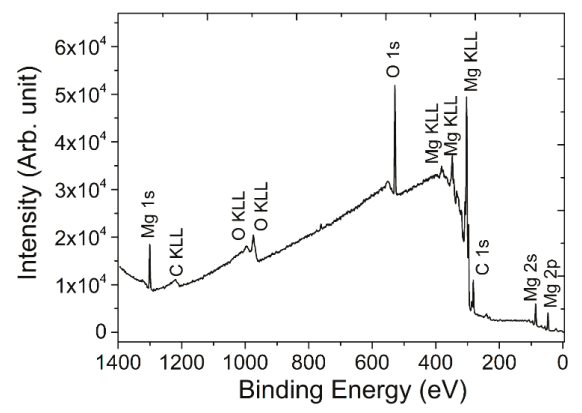

Figure 2. XPS survey spectra of a dense Mg film prepared for $\alpha=0^{\circ}$ and $P_{\text {Tot }}=0.26 \mathrm{~Pa}$. The sputtering power is $50 \mathrm{~W}$.

Table 1. Elemental composition of the as-deposited $\mathrm{Mg}$ thin film before and after $2 \mathrm{~min}$ of erosion.

\begin{tabular}{cccccc}
\hline \multicolumn{2}{c}{ At. $\%$ Mg } & \multicolumn{2}{c}{ At. \% O } & \multicolumn{2}{c}{ At. $\%$ C } \\
\hline As prepared & After erosion & As prepared & After erosion & As prepared & After erosion \\
\hline 44.6 & 89.9 & 48.9 & 10.1 & 10.5 & 0 \\
\hline
\end{tabular}

In order to support this conclusion, Figure 3 shows the evolution of the Mg2p XPS line as a function of the depth profiling. The estimated sputtering rate is $\sim 20 \mathrm{~nm} / \mathrm{min}$, according to the study reported by Milcius et al. [31]. For the as-deposited sample, it appears that the $\mathrm{Mg}$ line is composed of two components corresponding to metallic $\mathrm{Mg}$ at $49.5 \mathrm{eV}$ and $\mathrm{Mg}^{2+}$ at $50.8 \mathrm{eV}$. On the other hand, at $\sim 60 \mathrm{eV}$, a satellite line related to the metallic component is also observable. The presence of a strong oxidized component is in line with the surface stoichiometry of the surface composition of the as-prepared sample. After two minutes of sputtering, which is evaluated to correspond to $40 \mathrm{~nm}$, the oxidized component of the $\mathrm{Mg}$ peak completely vanishes while the satellite peak intensity strongly increases. Both these observations clearly confirm that the deposited film is only oxidized on its top surface.

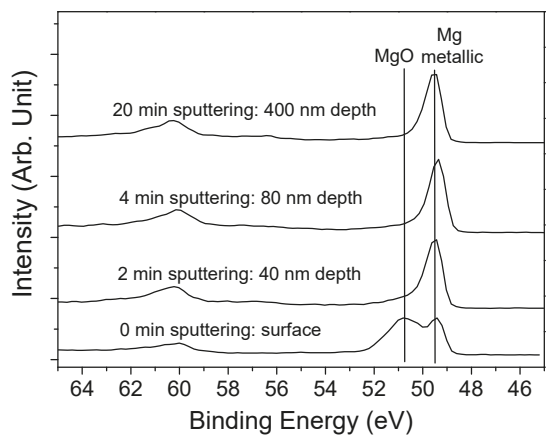

Figure 3. Evolution of the $\mathrm{Mg} 2 \mathrm{p}$ line during the depth profiling of the $\mathrm{Mg}$ films prepared for $\alpha=0^{\circ}$ and $P_{\text {Tot }}=0.26 \mathrm{~Pa}$. 
The XRD analysis of the as-deposited dense film is presented in Figure 4. The diffractogram reveals that the film consists of a polycrystalline material with the presence of several diffraction peaks, (002), (102), and (102), attributable to the cubic phase of Mg (JCPDS card N 04-0770) [31]. The (002) peak dominates the spectra which is likely explained by a preferential orientation of the growth along the $c$ axis as already reported for other materials [13]. In these conditions, applying the Sherrer formula to the dominating peak, a crystal size of about $25 \mathrm{~nm}$ has been calculated.

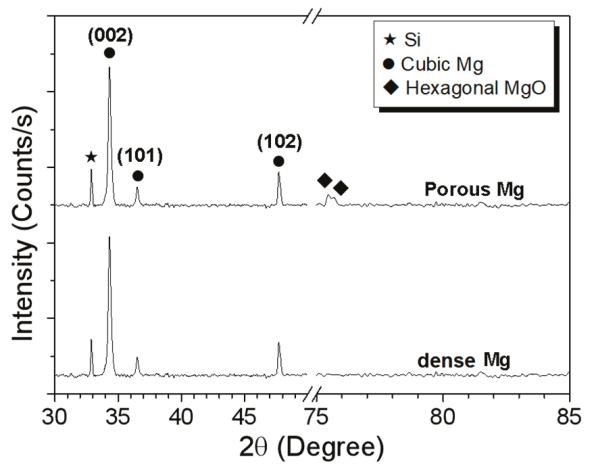

Figure 4. XRD spectra of the $\mathrm{Mg}$ films prepared for $\alpha=0^{\circ}$ and $P_{\mathrm{Tot}}=0.26 \mathrm{~Pa}$ (dense $\mathrm{Mg}$ ) and for $\alpha=85^{\circ}$ and $P_{\text {Tot }}=0.26 \mathrm{~Pa}$ (porous $\mathrm{Mg}$ ). In both cases, the sputtering power is $50 \mathrm{~W}$.

\subsection{Nano-Sculpted Mg Films}

After having characterized the reference film, we have studied the nanostructuration of the material by using MSGLAD by systematically studying the influence of both the tilt angle $(\alpha)$ and the working pressure $\left(P_{\mathrm{Tot}}\right)$ on the morphology of $\mathrm{Mg}$ films evaluated by SEM images. In addition, in order to clarify and understand the growth mechanisms, the experimental data was compared to the result of the modeling of the growth by kinetic Monte Carlo method using the NASCAM software.

Figure 5 shows, as a typical example of the generated microstructure, the cross section and the surface images of a $\mathrm{Mg}$ thin films deposited for $\alpha=85^{\circ}$ and $P_{\mathrm{Tot}}=0.26$. Pa. Figure 5 a shows the side view of the film which reveals that it is made of well separated faceted columns which have a width of $\sim 200 \mathrm{~nm}$, a length of $\sim 800 \mathrm{~nm}$, and (when considering the substrate surface) a tilt angle $\beta$ of $\sim 44^{\circ}$. The estimated dimension of the voids between the column is $\sim 10-60 \mathrm{~nm}$. Figure $5 \mathrm{~b}$ shows the surface of the film and confirms that the columns are well separated. Similar images for all samples synthesized by varying $\alpha$ and $P_{\text {Tot }}$ are shown in the Supplementary Figure S1. Similar morphologies have been obtained for all samples. Nevertheless, it is observed that the deposited film features ( $\beta$, the column length, the intercolumnar space, and the column width) more or less depend on these parameters. This offer many knobs for tuning the morphology of the deposited films as a function of the foreseen application.

Figure 6 summaries the evolution of $\beta$ for $60^{\circ}<\alpha<89^{\circ}$ (Figure $6 \mathrm{a}$ ) and for $0.13 \mathrm{~Pa}<P_{\mathrm{Tot}}<1.3 \mathrm{~Pa}$ (Figure 6b) evaluated from SEM images. Figure 6a reveals that $\beta$ strongly depends on $\alpha$ in line with previous work on $\mathrm{Ti}$ and $\mathrm{TiO}_{2}$ nano-sculpted films. This can be explained considering the influence of the atomic shadowing effects during deposition in GLAD configuration [19]. In particular, at extremely oblique incidence angles of the flux $\left(>60^{\circ}\right)$, the shadowing mechanism is strongly enhanced and results in a porous microstructure composed of columns inclined toward the vapor source [32]. $\beta$ drastically increases for $\alpha>60^{\circ}$ and stabilizes for $\alpha>85^{\circ}$. This evolution is explained by considering that when the direction of vapor incidence is normal to the film surface, the diffusion during the accommodation of the adatoms is a few atomic distances in the isotropic direction. However, under oblique incidence, the diffusion occurs in the direction given by the projection of the vapor beam direction on the film surface. The amount of kinetic energy (momentum) preserved in the direction parallel to the film surface is only determined by the angle of incidence. 

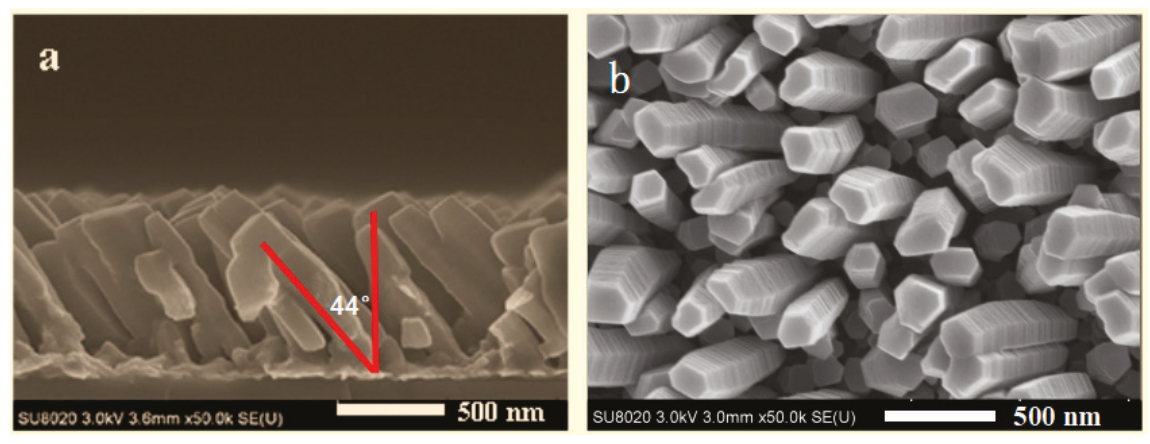

Figure 5. SEM (a) cross-section view and (b) surface view of a Mg nanostructured thin film synthesized for $\alpha=85^{\circ}$ and $P_{\text {Tot }}=0.26 \mathrm{~Pa}$.

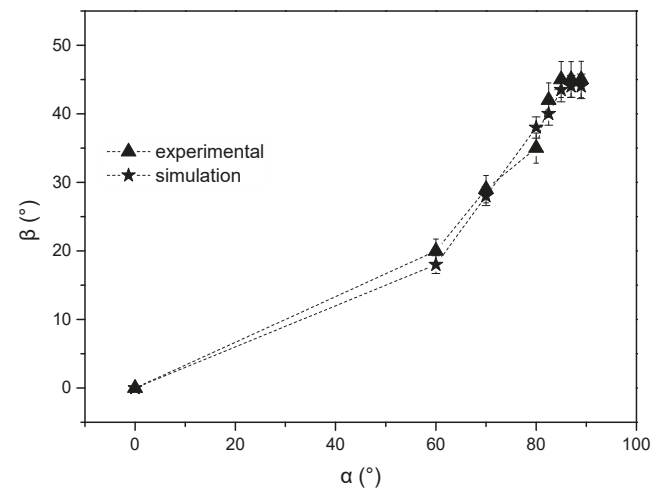

(a)

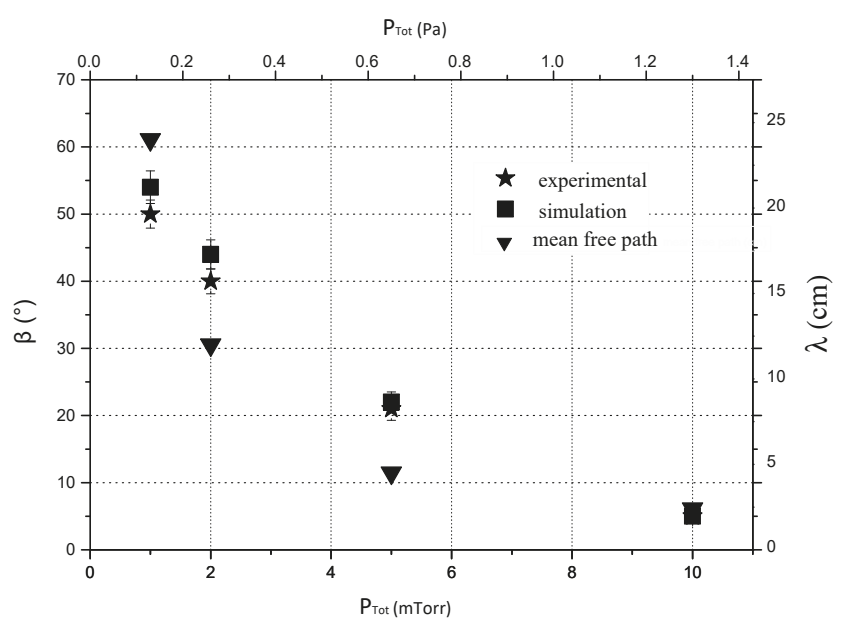

(b)

Figure 6. Evolution of the experimental and simulated values of the columnar angle $\beta$ as a function of the tilt angle, $\alpha$ (a) and of the working pressure, $P_{\text {Tot }}(\mathbf{b})$ for $\mathrm{Mg}$ nano-sculpted films and the mean free path. The films were deposited for a discharge power of $50 \mathrm{~W}$. 
It has to be noted that since the substrate to target distance $(8 \mathrm{~cm})$ is not very high in our chamber, the diameter of the target ( 2 inches) have to be taken into account to distinguish $\alpha$ and the incident angle of the particles, since the majority of the latter comes from the racetrack region of the target. The size of the particles source induces an angle of deviation in the $\alpha$ direction which increases with the target diameter [33]. This angle of deviation has been calculated in previous work for similar conditions and slightly increases with $\alpha$ due to the geometrical inclination of the substrate leading to an asymmetric deposition. Indeed, the particles sputtered at the left side of the target have a higher probability to reach the left side of the substrate and inversely for the right side. This increases the deviation angle and can explain the similar morphologies for coatings synthesized for $\alpha>85^{\circ}$.

On Figure 6a and Supplementary Figures S1 and S2, the corresponding kMC simulations obtained by using the NASCAM (NAnoSCAle Modeling) code and the procedure described in the experimental part. As input parameters, we have utilized the defined experimental parameters ( $50 \mathrm{~W}, 0.26 \mathrm{~Pa}$ ). A number of $5 \times 10^{5}$ atoms was chosen to obtain a film thickness similar to the one corresponding to the experimental conditions according to the size of the simulation box $(X=1000$ and $Y=2 \mathrm{Mg}$ atom unit). The ballistic deposition simulation of $\mathrm{Mg}$ atoms was used to understand the growth mechanisms of these films. The morphology of both simulated and experimental thin films were compared and the effect of the deposition parameters was analyzed. From the good agreement between the calculated and experimental data, it appears that the simulation employed in this work is perfectly adapted to our deposition.

Considering that our films are deposited without intentional heating, we can roughly estimate that the deposition temperature is about $\sim 323 \mathrm{~K}$. This corresponds to a $\mathrm{T}^{*}$, the generalized temperature of the Anders's Structure Zone Diagram (ASZM) [17], of $~ 0.25$ since the melting temperature of $\mathrm{Mg}$ is $923 \mathrm{~K}$. For this $\mathrm{T}^{*}$ condition and considering, in our process conditions, that the normalized energy was $<1$ [34], the ASZM depicts the synthesized films as a zone I film corresponding to $T_{s} / T_{m}<0.3$, for which surface diffusion is limited, and therefore does not allow for the filling of the void regions that form in the microstructure because of the geometrical shadowing effect occurring during the GLAD experiments. In these conditions, the film growth proceeds by the formation of an underdense, fine nanofibrous microstructure that develops into a columnar morphology. In the conditions, where geometric restrictions govern the formation of the microstructure, a strong anisotropic deposition is observed. Furthermore, it has to be noted that, for the "zone I" conditions, the columnar tilt angle ( $\beta=44 \pm 1.0^{\circ}$ in our case) is in line with the Tait's rule derived from geometric analysis of the inter-column shadowing geometry [34].

Figure $6 \mathrm{~b}$ shows the evolution of $\beta$ as a function of $P_{\text {Tot }}$. In this work, $P_{\text {Tot }}$ was varied from 0.13 to 1.3 Pa where $0.13 \mathrm{~Pa}$ corresponds to the minimum value necessary to maintain the magnetron discharge. The sputtering power and $\alpha$ were fixed at $50 \mathrm{~W}$ and $85^{\circ}$, respectively. $\beta$ rapidly decreases as $P_{\text {Tot }}$ increases, from $\beta=51 \pm 1.0^{\circ}$ for $0.13 \mathrm{~Pa}$ to $\beta=5 \pm 0.5^{\circ}$ for $1.3 \mathrm{~Pa}$. The modification of the columnar tilt angle can be attributed to a decrease of the collimation of the incident particle flux due to the increase of collision probability as $P_{\text {Tot }}$ increases. Indeed, this probability mainly depends on the mean free path of the sputtered $\mathrm{Mg}$ atoms $\left(\lambda_{\mathrm{Mg}}\right)$ which is inversely proportional to $P_{\text {Tot }}$ following this equation:

$$
\lambda=\frac{\kappa_{B} T}{\sqrt{2} \pi d^{2} P_{T o t}}
$$

where, $\kappa_{B}$ is the Boltzmann constant $\left(1.380 \times 10^{-23} \mathrm{~J} / \mathrm{K}\right), T$ is the temperature in $K, P_{\text {Tot }}$ is the total pressure in Pascal, and $d$ is the diameter of the gas particles in meters. From this relation, $\lambda_{\mathrm{Mg}}$ (atomic diameter $=1.72 \AA$ ) ranges from 24 to $2.4 \mathrm{~cm}$ between 0.13 and $1.33 \mathrm{~Pa}$, respectively. Considering the target/substrate distance $(8 \mathrm{~cm})$ that is used, an increase of $P_{\text {Tot }}$ induces a large amount of collisions between particles for the 1.3 Pa conditions resulting in a less porous film. Figure $6 \mathrm{~b}$ shows that the morphology of the simulated thin films is again in line with the experimental ones. In addition, the calculated mean free path for Ti atoms as a function of the pressure is also presented. It appears that the mean free path becomes smaller than the target-to-substrate distance for a pressure value around 
$0.7 \mathrm{~Pa}\left(\lambda_{\mathrm{Mg}}=4.5 \mathrm{~cm}\right)$. Below this pressure, very few collisions occur through the vapor phase, whereas, a higher pressure leads to numerous collisions between particles. The analysis of the predicted film morphology at different pressures also allows determination of the range of pressure where a ballistic deposition process occurs which, in our case, is between 0.13 and $0.26 \mathrm{~Pa}$, and its mean free path ranges from $24 \mathrm{~cm}$ to $12 \mathrm{~cm}$.

If it is very difficult to measure the porosity of thin films $(\chi)$ experimentally because of the low quantity of material. Therefore, it has been simulated using the PoreSTAT software [35] which uses the NASCAM files to perform a full 3D analysis of the porous structure of the material, or a 2D study on the different slices of material belonging to the $\mathrm{XZ}$ or $\mathrm{YZ}$ planes $(\mathrm{X}$ and $\mathrm{Y}$ are horizontal axes defining the substrate, whereas, $Z$ corresponds to the vertical axe, defining the film height) [36]. Figure 7 shows the evolution of $\chi$ as a function of $\alpha$ for $P_{\text {Tot }}=0.26 \mathrm{~Pa}$ and of $P_{\text {Tot }}$ for $\alpha=85^{\circ}$. It appears that $\chi$ increases with $\alpha$ from $54 \%$ until $60 \%$ for $\alpha=85^{\circ}$ and then stabilizes for higher values. On the other hand, $\chi$ increases from $51 \%$ until $66 \%$ when reducing $P_{\text {Tot }}$ from 1.33 to $0.13 \mathrm{~Pa}$. These evolutions are obviously correlated with the evolution of the nanosculpted films feature with the $\alpha$ and $P_{\text {Tot }}$ parameters. More precisely, it appears from Figure 8 that the evolution of $\chi$ is linearly correlated with the evolution of the aspect ratio, $\Gamma$, which is convenient since tuning the key parameters of the process such as $\alpha$ or $P_{\text {Tot, }}$, we get a fine control on the porosity of the films which is indirectly correlated with the surface area of the material. This linear correlation can be understood by considering the meaning of $\Gamma$ which is the ratio of the inter columnar space on the width of columns. On the basis of this definition, it is obvious that an increase of $\Gamma$ will lead to an increase of the material porosity since there is more space between the columns because the intercolumnar space increases or the column width reduces (or both).

The chemical and structural characterization of the nano-sculpted films have again been performed by XPS and XRD measurements. As expected the XPS data reveals the presence of strong oxygen and carbon signals explained by the surface contamination which is likely even stronger for these porous films. Unfortunately, in this case, because of the nanostructured features of the material, it is not easy to depth profile the thin film. To the contrary, XRD measurements are still possible in good conditions and are reported in Figure 2 for a nano-sculpted sample synthesized for $\alpha=85^{\circ}$ and $P_{\text {Tot }}=0.26 \mathrm{~Pa}$ (sputtering power of $50 \mathrm{~W}$ ).

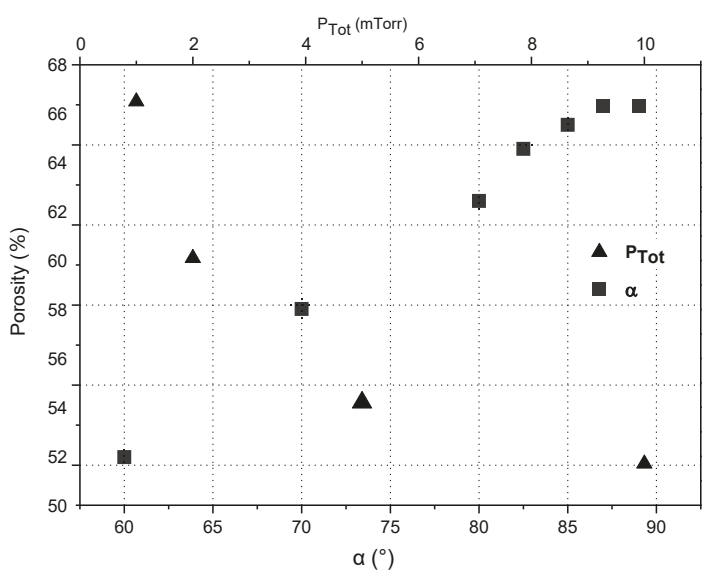

Figure 7. Evolution of the simulated values of the porosity as a function of the tilt angle, $\alpha$, and of the working pressure, $P_{\mathrm{Tot}}$, for $\mathrm{Mg}$ nano-sculpted films prepared using a discharge power of $50 \mathrm{~W}$. 


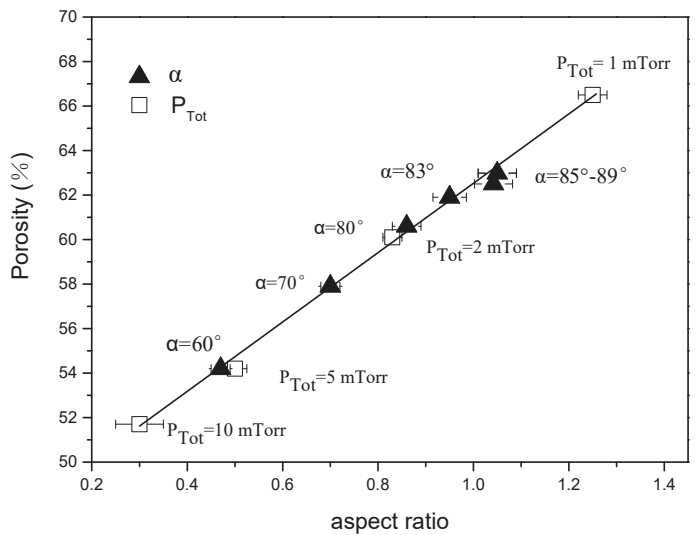

Figure 8. Evolution of the porosity as a function of the aspect ratio, $\Gamma$, for $\mathrm{Mg}$ nano-sculpted films with the $\alpha$ and $P_{\text {Tot }}$.

From the data we determine that the crystalline constitution of the material is only slightly affected by the utilization of the GLAD geometry. Indeed, all diffraction peaks observed for the dense Mg coatings are again present with the same relative intensity. The only minor difference is related to the presence of $\mathrm{MgO}$ lines that appear in addition to the already identified $\mathrm{Mg}$ lines. This suggests that the quantity of oxygen in the bulk of the material is likely higher in the nano-sculpted films allowing for the presence of $\mathrm{MgO}$ grains. This can be understood when considering the magnified surface area which is subjected to oxidation during the growth as compared with the situation occurring for dense film deposition.

\section{Conclusions and Perspectives}

Through the present work, we provide a fairly clear description and understanding of a magnetron sputtering in grazing angle geometry method allowing for the deposition of $\mathrm{Mg}$ nanocolumnar thin films for potential hydrogen storage. The effect of the deposited angle and sputtering pressure on the $\mathrm{Mg}$ nanocolumnar structure has been specifically investigated. The good agreement between experimental observations and model predictions indicates that the simulations realistically reproduces the competitive growth mechanism involved in GLAD experiments. On the basis of this study, we conclude that the fundamental mechanisms responsible for the growth of nano-sculpted $\mathrm{Mg}$ film in a MSGLAD are based on (i) the self-shadowing mechanisms at the surface and (ii) the collisional processes of the sputtered particles in the gas phase. In addition, it appears, under our experimental conditions, that the self-diffusion of deposited $\mathrm{Mg}$ atoms is strongly reduced and that the microstructure of our films belong to the zone I of the ASZM. In addition, we learn that when growing Mg porous films and because of the strong reactivity of $\mathrm{Mg}$ towards oxygen, surface and even bulk oxidation easily occurs. If not controlled, probably, the porous film would not be suitable for hydrogen storage.

Supplementary Materials: The following are available online at http://www.mdpi.com/2079-6412/9/6/361/s1, Figure S1: SEM cross-section view and of $\mathrm{Mg}$ films deposited for $P_{\mathrm{Tot}}=0.26 \mathrm{~Pa}$ and varying $\alpha$ from $60^{\circ}$ to $89^{\circ}$. The green images correspond to the structures calculated by using Mkc modeling, Figure S2: SEM cross-section view and of $\mathrm{Mg}$ films deposited for $\alpha=85^{\circ}$ and varying $P_{\text {Tot }}$ from 0.13 to $1.3 \mathrm{~Pa}$. The green images correspond to the structures calculated by using Mkc modeling.

Author Contributions: Conceptualization, H.L. and R.S.; Methodology, H.L.; Software, H.L.; validation, H.L., X.G., A.P. and D.T.; Formal analysis, H.L.; Investigation, H.L.; Resources, H.L.; Data curation, H.L.; Writing-original draft preparation, H.L.; Writing-review and editing, R.S. and W.L.; Visualization, H.L.; Supervision, R.S. and W.L.; Project administration, R.S., W.L. and M.C.; Funding acquisition, W.L. and M.C.; The design of the study, H.L., R.S.; The collection, analyses or interpretation of data, H.L., X.G., A.P. and D.T.; The writing of the manuscript, H.L.; The decision to publish the results, R.S., W.L. and M.C. 
Funding: This research was funded by the F.R.I.A grant of the National Fund for Scientific Research (FNRS, Belgium); the Joint Foundation of National Natural Science Foundation of China, Grant No. U1764254; the National Natural Science Foundation of China, Grant No. 51871166; 321 talent projects of Nanjing (China), Grant No. 631783; 111 Project (China), D17003.

Acknowledgments: A.P. thanks the F.R.I.A grant of the National Fund for Scientific Research (FNRS, Belgium); H.L. thanks the Joint Foundation of National Natural Science Foundation of China (Grant No. U1764254); the National Natural Science Foundation of China (Grant No. 51871166); 321 talent projects of Nanjing (Grant No. 631783), China; 111 Project (D17003), China.

Conflicts of Interest: The authors declare no conflict of interest.

\section{References}

1. Chaubey, R.; Sahu, S.; James, O.O.; Maity, S. A review on development of industrial processes and emerging techniques for production of hydrogen from renewable an sustainable sources. Renew. Sustain. Energy Rev. 2013, 23, 443-462. [CrossRef]

2. Momirlan, M.; Veziroglu, T.N. The properties of hydrogen as fuel tomorrow in sustainable energy system for a cleaner planet. Int. J. Hydrog. Energy 2005, 30, 795-802. [CrossRef]

3. Haas, I.; Gedanken, A. Synthesis of metallic magnesium nanoparticles by sono electrochemistry. Chem. Commun. 2008, 15, 1795-1797. [CrossRef] [PubMed]

4. Jong, H.D. The preparation of carbon-supported magnesium nanoparticles using melt infiltration. Chem. Mater. 2007, 19, 6052-6057. [CrossRef]

5. Kooi, B.J.; Palasantzas, G.; De Hosson, J.T.M. Gas-phase synthesis of magnesium nanoparticles: A high-resolution transmission electron microscopy study. Appl. Phys. Lett. 2006, 89, 161914. [CrossRef]

6. Aneke, M.; Wang, M. Energy storage technologies and real life applications-A state of the art review. Appl. Energy 2016, 179, 350-377. [CrossRef]

7. Basak, S.; Shashikala, K.; Kulshreshth, S.K. Hydrogen absorption characteristics of Zr sustituted $\operatorname{Ti}_{0.85} \mathrm{VFe}_{0.15}$ alloy. Int. J. Hydrog. Energy 2008, 33, 350-355. [CrossRef]

8. Suh, M.P.; Park, H.J.; Prasad, T.K.; Lim, D.W. Hydrogen storage in metal-organic framworks. Chem. Rev. 2012, 112, 782-835. [CrossRef]

9. Li, W.; Li, C.; Ma, H.; Chen, J. Magnesium nanowires: enhanced kinetics for hydrogen absorption and desorption. J. Am. Chem. Soc. 2007, 129, 6710-6711. [CrossRef]

10. Sun, Y.H.; Shen, C.Q.; Lai, Q.W.; Liu, W.D.; Wang, W.; Francois, K.; Zinsoua, A. Tailoring magnesium based materials for hydrogen storage through synthesis: Current state of the art. Energy Storage Mater. 2017, 10, 168-198. [CrossRef]

11. Jain, I.P.; Lal, C.; Jain, A. Hydrogen storage in Mg: A most promising material. Int. J. Hydrog. Energy 2010, 35, 5133-5144. [CrossRef]

12. Sadhasivam, T.; Kim, H.T.; Jung, S.; Roh, S.H.; Park, J.H.; Jung, H.Y. Dimensional effects of nanostructured $\mathrm{Mg} / \mathrm{MgH}_{2}$ for hydrogen storage applications: A review. Renew. Sustain. Energy Rev. 2017, 72, 523-534. [CrossRef]

13. Jeon, K.J.; Moon, H.R.; Ruminski, A.M.; Jiang, B.; Kisielowski, C.; Bardhan, R. Air-stable magnesium nanocomposites provide rapid and high-capacity hydrogen storage without using heavy-metal catalysts. Nat. Mater. 2011, 4, 286-290. [CrossRef] [PubMed]

14. Yuan, J.G.; Zhu, Y.F.; Li, Y.; Li, L.Q. Effect of multi-wall carbon nanotubes supported palladium addition on hydrogen storage properties of magnesium hydride. Int. J. Hydrog. Energy 2014, 39, 10184-10194. [CrossRef]

15. Barawi, M.; Granero, C.; Chao, P.D.; Manzano, C.V.; Gonzalez, M.; Jimenez, R.D.; Ferrer, I.J.; Ares, J.R.; Fernánde, J.F.; Sanchez, C. Thermal decomposition of noncatalysed $\mathrm{MgH}_{2}$ film. Int. J. Hydrog. Energy 2014, 39, 9865-9870. [CrossRef]

16. Dura, J.A.; Kelly, S.T.; Kienzle, P.A.; Her, J.H.; Udovic, T.J.; Majkrzak, C.F.; Chung, C.J.; Clemens, B.M. Porous $\mathrm{Mg}$ formation upon dehydrogenation of $\mathrm{MgH}_{2}$ thin films. J. Appl. Phys. 2011, 109, 093501. [CrossRef]

17. Laforge, J.M.; Taschuk, M.T.; Brett, M.J. Glancing angle deposition of crystalline zinc oxide nanorods. Thin Solid Films 2011, 519, 3530-3537. [CrossRef]

18. Thiry, D.; Aparicio, F.J. Surface temperature: A key parameter to control the propanethiol plasma polymer chemistry. J. Vac. Sci. Technol. A 2014, 32, 050602. [CrossRef] 
19. Dervaux, J.; Cormier, P.A.; Konstantinidis, S.; Di, R.; Coulembier, O.; Dubois, P.; Snyders, R. Deposition of porous titanium oxide thin films as anode material for dye sensitized solar cells. Vacuum 2015, 114, 213-220. [CrossRef]

20. Dervaux, J.; Cormier, P.A.; Konstantinidis, S.; Moskovkin, P.; Lucas, S.; Snyders, R. Nanostructured Ti thin films by combining GLAD and magnetron sputtering and did a joint experimental and modeling study. In Proceedings of the 22nd International Symposium on Plasma Chemistry, Antwerp, Belgium, 5-10 July 2015.

21. Huo, H.W.; Li, Y.; Wang, F.H. Preparation and corrosion resistance of magnesium coatings by magnetron sputtering deposition. J. Mater. Sci. Technol. 2003, 19, 459-462.

22. Saraiva, M.; Depla, D. Texture and microstructure in co-sputtered Mg-M-O (M = Mg, Al, Cr, Ti, Zr and Y) films. J. Appl. Phys. 2012, 111, 104903. [CrossRef]

23. Park, C.H.; Lee, W.G.; Kim, D.H.; Ha, H.J.; Ryu, J.Y. Surface discharge characteristics of MgO thin films prepared by RF reactive magnetron sputtering. Surface Coat. Technol. 1998, 110, 128-135. [CrossRef]

24. Yao, H.B.; Li, Y.; Wee, A.T.S. An XPS investigation of the oxidation/corrosion of melt-spun Mg. Appl. Surf. Sci. 2000, 158, 112-119. [CrossRef]

25. Scherrer, P. Bestimmung der Grösse und der inneren Struktur von Kolloidteilchen mittels Röntgenstrahlen. Nachr. Ges. Wiss. Göttingen 1918, 26, 98-100.

26. Lucas, S. Simulation at high temperature of atomic deposition, islands coalescence, Ostwald and inverse ripening with a general simple kinetic Monte Carlo code. Thin Solid Films 2010, 518, 5355-5361. [CrossRef]

27. Window, B. Recent advances in sputter deposition. Surf. Coat. Technol. 1995, 71, 91-93. [CrossRef]

28. Zhong, P.; Que, W.; Zhang, J.; Jia, Q.; Wang, W.; Liao, Y. Charge transport and recombination in dye-sensitized solar cells based on hybrid films of $\mathrm{TiO}_{2}$ particles/ $\mathrm{TiO}_{2}$ nanotube. J. Alloys Compd. 2011, 509, 7803-7808. [CrossRef]

29. Ruminski, A.M.; Bardhan, R.; Brand, A.; Aloni, S.; Urban, J.J. Synergistic enhancement of hydrogen storage and air stability via Mg nanocrystal-polymer interfacial interactions. Energy Environ. Sci. 2013, 6, 3267-3271. [CrossRef]

30. Snyders, R.; Gouttebaron, R.; Dauchota, J.P.; Hecq, M. Mass spectrometry diagnostic of dc magnetron reactive sputtering. J. Anal. At. Spectrom. 2003, 18, 618-623. [CrossRef]

31. Milcius, D.; Grbović-Novaković, J.; Zostautienė, R.; Lelis, M.; Girdzevicius, D.; Urbonavicius, M. Combined XRD and XPS analysis of ex-situ and in-situ plasma hydrogenated magnetron sputtered Mg films. J. Alloys Compd. 2015, 647, 790-796. [CrossRef]

32. Mor, G.K.; Shankar, K.; Paulose, M.; Varghese, O.K.; Grimes, C.A. Use of highly-ordered $\mathrm{TiO}_{2}$ nanotube arrays in dye-sensitized solar cells. Nano Lett. 2006, 6, 215-218. [CrossRef]

33. Pozuelo, M.; Melnyk, C.; Kao, W.H.; Yang, J.M. Cryomilling and spark plasma sintering of nanocrystalline magnesium-based alloy. J. Mater. Res. 2011, 14, 904-911. [CrossRef]

34. Tait, R.N.; Smy, T.; Brett, M.J. Modelling and characterization of columnar growth in evaporated films. Thin Solid Films 1993, 226, 196-201. [CrossRef]

35. PoreSTAT. Available online: http://nanoscops.icmse.csic.es/software/porestat (accessed on 31 March 2017).

36. Godinho, V.; Hernández, J.C.; Jamon, D.; Rojas, T.C.; Schierholz, R.; García-López, J.; Ferrer, F.J.; Fernández, A. A new bottom-up methodology to produce silicon layers with a closed porosity nanostructure and reduced refractive index. Nanotechnology 2013, 24, 275604. [CrossRef]

(C) 2019 by the authors. Licensee MDPI, Basel, Switzerland. This article is an open access article distributed under the terms and conditions of the Creative Commons Attribution (CC BY) license (http://creativecommons.org/licenses/by/4.0/). 
Article

\title{
Phase Selectivity in $\mathrm{Cr}$ and $\mathrm{N}$ Co-Doped $\mathrm{TiO}_{2}$ Films by Modulated Sputter Growth and Post-Deposition Flash-Lamp-Annealing
}

\author{
Raúl Gago ${ }^{1, *}$, Slawomir Prucnal ${ }^{2}$, René Hübner ${ }^{2}$, Frans Munnik ${ }^{2}$, David Esteban-Mendoza ${ }^{1}$, \\ Ignacio Jiménez ${ }^{1}$ and Javier Palomares ${ }^{1}$ \\ 1 Instituto de Ciencia de Materiales de Madrid, Consejo Superior de Investigaciones Científicas, \\ E-28049 Madrid, Spain \\ 2 Helmholtz-Zentrum Dresden-Rossendorf, Institute of Ion Beam Physics and Materials Research, \\ D-01328 Dresden, Germany \\ * Correspondence: rgago@icmm.csic.es; Tel.: +34-91-334-9090
}

Received: 11 June 2019; Accepted: 14 July 2019; Published: 17 July 2019

\begin{abstract}
In this paper, we report on the phase selectivity in $\mathrm{Cr}$ and $\mathrm{N}$ co-doped $\mathrm{TiO}_{2}\left(\mathrm{TiO}_{2}: \mathrm{Cr}, \mathrm{N}\right)$ sputtered films by means of interface engineering. In particular, monolithic $\mathrm{TiO}_{2}: \mathrm{Cr}, \mathrm{N}$ films produced by continuous growth conditions result in the formation of a mixed-phase oxide with dominant rutile character. On the contrary, modulated growth by starting with a single-phase anatase $\mathrm{TiO}_{2}: \mathrm{N}$ buffer layer, can be used to imprint the anatase structure to a subsequent $\mathrm{TiO}_{2}: \mathrm{Cr}, \mathrm{N}$ layer. The robustness of the process with respect to the growth conditions has also been investigated, especially regarding the maximum $\mathrm{Cr}$ content $(<5$ at. $\%)$ for single-phase anatase formation. Furthermore, post-deposition flash-lamp-annealing (FLA) in modulated coatings was used to improve the as-grown anatase $\mathrm{TiO}_{2}: \mathrm{Cr}, \mathrm{N}$ phase, as well as to induce dopant activation ( $\mathrm{N}$ substitutional sites) and diffusion. In this way, $\mathrm{Cr}$ can be distributed through the whole film thickness from an initial modulated architecture while preserving the structural phase. Hence, the combination of interface engineering and millisecond-range-FLA opens new opportunities for tailoring the structure of $\mathrm{TiO}_{2}$-based functional materials.
\end{abstract}

Keywords: oxide materials; doping; sputter deposition; modulated growth; flash-lamp-annealing; XANES

\section{Introduction}

Titania or titanium dioxide $\left(\mathrm{TiO}_{2}\right)$ is a functional wide band-gap semiconductor with tuneable electrical and optical properties by intrinsic (structure in single- or mixed-phase anatase/rutile and/or native defects) or extrinsic (doping) mechanisms [1]. The relevance of doping effects comprises many applications that partially rely on the performance of $\mathrm{TiO}_{2}$ as a solvent for impurities. In the case of cation dopants, metal incorporation has been used to functionalize or enhance $\mathrm{TiO}_{2}$ as photocatalyst [2], diluted magnetic semiconductor [3], or transparent conductor material [4].

One of the most interesting properties of $\mathrm{TiO}_{2}$ relies on its photoactivity, which has been exploited in many applications, such as photocatalysis, hydrogen production, pigments or solar cells [2,5]. However, due to the relatively large band-gap of $\mathrm{TiO}_{2}(>3 \mathrm{eV})$, its photoactivity is limited to the ultraviolet (UV) region of the solar spectrum (only $5 \%$ of the total energy [6]). Therefore, many efforts have been focused on band-gap narrowing for $\mathrm{TiO}_{2}$ to achieve a visible-light (VISL) response. Such a challenge is mainly realized via doping with foreign atoms at cation or anion sites [2]. In this respect, non-metal (anion) doping has been extensively studied, especially after the work by Asahi et al. [7] on nitrogen $(\mathrm{N})$ doped $\mathrm{TiO}_{2}\left(\mathrm{TiO}_{2}: \mathrm{N}\right)$, where the effective optical absorption appears to be related 
with intragap localized states [8]. In addition, the solid solubility of $\mathrm{N}$ in $\mathrm{TiO}_{2}$ is rather low, and this situation leads to excess $\mathrm{N}$ in (a priori, undesirable) interstitial positions [7]. These sites not only compromise the effectiveness of band-gap narrowing, but provide recombination centres responsible for the loss of photo-generated electron-hole pairs [4]. Metal (cation) doping represents another approach to increase VISL absorption in doped $\mathrm{TiO}_{2}$ [9], but it induces structural distortions in the host matrix and the defects act as carrier recombination centres [7]. Among these systems, Cr-doped $\mathrm{TiO}_{2}\left(\mathrm{TiO}_{2}: \mathrm{Cr}\right)$ has been addressed due to its catalytic [10,11] and magnetic [12] properties. A recent concept for effective band-gap narrowing relies on simultaneous doping of $\mathrm{TiO}_{2}$ with anions $(\mathrm{C}, \mathrm{N}$, etc.) and transition metals $(\mathrm{Cr}, \mathrm{V}, \mathrm{Mo}$, etc.). In such a case, the opposite charge states of $\mathrm{p}$ - and n-type sites in non-compensated dopants (e.g., $\mathrm{Cr} / \mathrm{N}$ ) should significantly increase the solubility limit of dopant pairs [13]. Based on this hypothesis, $\mathrm{Cr}-\mathrm{N}$ co-doped $\mathrm{TiO}_{2}\left(\mathrm{TiO}_{2}: \mathrm{Cr}, \mathrm{N}\right)$ nanoparticles [6] and single-crystal anatase [14] or rutile [15] thin films have been produced with an extraordinary reduction of the bandgap. The experimental results also indicated that (substitutional) $\mathrm{Cr}$ and $\mathrm{N}$ dopants are coupled due to the preferential formation of $\mathrm{Cr}-\mathrm{N}$ bonds [6,14].

In this work, we address the structural impact of co-doping in $\mathrm{TiO}_{2}: \mathrm{Cr}, \mathrm{N}$ films produced by magnetron sputtering. However, $\mathrm{Cr}$ containing $\mathrm{TiO}_{2}$ samples produced by this method typically have a poor crystalline quality even under growth on heated substrates up to $500{ }^{\circ} \mathrm{C}$ [16]. This eventual drawback represents a handicap with respect to other methods producing $\mathrm{TiO}_{2}: \mathrm{Cr}, \mathrm{N}$ films with high structural quality $[13,14]$. Post-deposition treatments can be used to enhance the structural quality where, additionally, further dopant activation or the promotion of a specific structure can be achieved. For industry-oriented applications, non-contact and rapid treatments pose a great advantage. Among such methods, millisecond-range flash-lamp-annealing (FLA) [8] enables the control of dopant diffusion and activation, where only the near-surface region is annealed and rapidly cooled. Recently, we reported [17] the structural impact of $\mathrm{Cr}$ incorporation before and after $\mathrm{FLA}$ in $\mathrm{TiO}_{2}$ films grown by magnetron sputtering. We found that $\mathrm{TiO}_{2}$ phases can accommodate up to $\sim 5$ at. $\% \mathrm{Cr}$ and, in general, rutile environments are favored. This situation may not be desirable for certain applications, since the anatase phase shows a substantially higher photoactivity than rutile [18]. However, phase selectivity in $\mathrm{TiO}_{2}$-based films is a complex competition between nucleation and growth mechanisms. Rutile is the most stable and dense structure, whereas anatase is a metastable phase. The nucleation of rutile requires more energy input than anatase, but after nucleation it grows more easily within a wider range of conditions than anatase [19].

The objectives of this work are two-fold. First, we aim to extend our previous structural study to co-doped films. Second, we seek growth or processing conditions that yield preferential formation of the anatase phase. Since, as stated above, the growth regime for anatase seems to be the limiting factor, we test the preparation of modulated architectures as an attempt to transfer and retain the growth of the anatase phase in the $\mathrm{Cr}$-containing layer. Remarkably, this approach has been found to be rather successful. In addition, FLA has been used to improve the quality of the resulting phase and to induce dopant activation and diffusion. The present results provide a relevant framework for tailoring the atomic structure of $\mathrm{TiO}_{2}$-based mixed oxides by exploiting the concept of interface engineering in combination with FLA.

\section{Materials and Methods}

\subsection{Sample Preparation}

In this work, $\mathrm{TiO}_{2}: \mathrm{Cr}, \mathrm{N}$ coatings were grown by reactive magnetron sputtering on p-type ( $\mathrm{B}$ doped) commercial Si(100) substrates (University Wafer, South Boston, MA, USA) cleaved into $12 \times 12 \mathrm{~mm}^{2}$ pieces. Individual 3" Ti and Cr targets (99.99\% purity, Testbourne Ltd., Basingstoke, UK) were used for co-sputtering, both located at $\sim 15 \mathrm{~cm}$ from the grounded substrates in a confocal geometry at an angle of $30^{\circ}$ with respect to the substrate normal. The base pressure in the deposition chamber was $10^{-4} \mathrm{~Pa}$. The working pressure was $0.5 \mathrm{~Pa}$ with a gas (99.9995\% purity grade) mixture of $\mathrm{Ar}(50 \%), \mathrm{N}_{2}$ 
(44\%) and $\mathrm{O}_{2}(6 \%)$ set by individual mass flow controllers. For plasma generation, a DC signal with a power of $150 \mathrm{~W}$ was applied to the Ti cathode, whereas the power applied to the $\mathrm{Cr}$ cathode, $W_{\mathrm{Cr}}$, was modulated from 0 to $25 \mathrm{~W}$ according to the desired coating architecture. In all cases, the growth was carried out for $2 \mathrm{~h}$, resulting in a total thickness of $\sim 100 \mathrm{~nm}$. Monolithic and modulated film architectures were studied. In the first case, $W_{\mathrm{Cr}}$ was kept constant during the whole deposition time. In the latter case, a buffer $\mathrm{TiO}_{2}: \mathrm{N}$ films was initially grown $\left(\mathrm{W}_{\mathrm{Cr}}=0 \mathrm{~W}\right)$ with a thickness up to several tens of nm's followed by the subsequent instant (bilayer coating) or gradual (gradient coating) increase in $W_{\mathrm{Cr}}$ to produce the Cr-containing layer(s). The deposition was done at a substrate temperature, $T_{\mathrm{S}}$, of $300{ }^{\circ} \mathrm{C}$ to achieve high-quality anatase phase in $\mathrm{TiO}_{2}: \mathrm{N}$ films [20].

The as-grown samples were cut in two and one piece was kept as a reference while the other was processed with FLA for $20 \mathrm{~ms}$ at a continuous flow of $\mathrm{N}_{2}$ ( $99.999 \%$ purity). The overall energy density was set to $\sim 70 \mathrm{~J} / \mathrm{cm}^{2}$ according to the optimum conditions for anatase crystallization from (amorphous) pure $\mathrm{TiO}_{2}$ films [17]. Such FLA condition corresponds to a maximum surface temperature in the range of $1100{ }^{\circ} \mathrm{C}$. The heating and cooling rate during millisecond FLA is in the range of $100 \mathrm{~K} / \mathrm{ms}$ and $200 \mathrm{~K} / \mathrm{s}$, respectively. Further details about the FLA system can be found in [21].

\subsection{Sample Characterization}

The composition profile of the $\mathrm{TiO}_{2}: \mathrm{Cr}, \mathrm{N}$ layers was determined by Rutherford backscattering spectrometry (RBS). The measurements were performed at the Ion Beam Center (IBC) of Helmholtz-Zentrum Dresden-Rossendorf (HZDR) using a $1.7 \mathrm{MeV} \mathrm{He}^{+}$probing beam. The RBS spectra were acquired under normal incidence with a "random" scan (to avoid channelling effects in the substrate signal), and the backscattered particles were detected with a silicon detector at a scattering angle of $165^{\circ}$. For quantitative analysis, the RBS spectra were simulated with the SIMNRA code [22]. Complementary compositional analysis regarding light elements was done by means of heavy-ion elastic recoil detection analysis (ERDA). The measurements were carried out with a $35 \mathrm{MeV}$ $\mathrm{Cl}^{7+}$ beam impinging at $75^{\circ}$ with respect to the sample surface normal. The scattered ions and recoils were detected with a Bragg ionization chamber (BIC) located at a scattering angle of $31^{\circ}$. The BIC chamber allows for the discrimination of detected particles according to their atomic number $(Z)$. The analysis of the recoil spectra and scattered $\mathrm{Cl}$ spectrum was performed simultaneously for each sample with the code NDF [23].

The phase structure of the samples was examined by grazing-incidence X-ray diffraction (GI-XRD) measurements using a D5000 (BRUKER AXS, Billerica, MA, USA) diffractometer with $\mathrm{Cu}-\mathrm{K} \alpha$ radiation (wavelength of $1.5418 \AA$ ). The data were collected at an incidence angle of $0.5^{\circ}$. The local bonding structure with element sensitivity was studied by X-ray absorption near-edge structure (XANES) analysis [24] with soft X-rays. XANES provides short-range information of electronic states for each individual element, being a powerful technique to study complex multi-element systems. Moreover, the technique can be applied to materials with either amorphous or crystalline structure. Particularly, $\mathrm{TiO}_{2}$-based materials have been widely studied by XANES, providing clear distinct spectral fingerprints of $\mathrm{TiO}_{2}$ polymorphs that can be used for univocal phase identification [25]. XANES measurements were carried out at the dipole beamline PM3 of the synchrotron facility BESSY-II of Helmholtz-Zentrum Berlin (HZB). The data were acquired using the ALICE endstation in the total electron yield (TEY) mode. TEY-XANES probes the near-surface region (up to a few tens of nm's) and, therefore, in-depth structural information can be achieved by the combination of XANES and XRD. The electronic structure has also been studied by high-resolution X-ray photoelectron spectroscopy (XPS). The spectra were acquired with a Phoibos 150 spectrometer (SPECS, Berlin, Germany) equipped with a hemispherical analyser and 2D-DLD detector. The measurements were performed with monochromatic $\mathrm{Al} \mathrm{K} \alpha$ radiation at normal emission take-off angle. The spectra were acquired using an energy step of $0.05 \mathrm{eV}$ and pass-energy of $10 \mathrm{eV}$, providing an overall instrumental peak broadening of $\sim 0.4 \mathrm{eV}$.

The microstructure of the $\mathrm{TiO}_{2}: \mathrm{Cr}, \mathrm{N}$ thin films was analysed locally with cross-sectional transmission electron microscopy (TEM) using an image $\mathrm{C}_{\mathrm{s}}$-corrected Titan 80-300 microscope 
(FEI, Eindhoven, Netherlands) operated at an accelerating voltage of $300 \mathrm{kV}$. TEM specimens were prepared by sawing, grinding, dimpling, and finally, $\mathrm{Ar}^{+}$ion-milling. In particular, bright-field TEM imaging and selected-area electron diffraction (SAED) were performed. Since the smallest available selected area aperture of $10 \mu \mathrm{m}$ covers a circular area with a diameter of about $190 \mathrm{~nm}$, amorphous glue used for TEM specimen preparation contributes to the SAED patterns recorded. Complementary information was extracted from high-angle annular dark-field scanning transmission electron microscopy (HAADF-STEM) imaging and spectrum imaging analysis based on energy-dispersive X-ray spectroscopy (EDXS). This analysis was performed with a Talos F200X microscope (FEI, Brno, Czech Republic) operated at $200 \mathrm{kV}$ and equipped with a Super-X EDXS detector system. The TEM specimens were placed for $8 \mathrm{~s}$ into a Model 1020 Plasma Cleaner (Fischione, Export, PA, USA) to remove organic surface contamination before the analysis.

\section{Results and Discussion}

\subsection{Compositional Profile in Monolithic and Modulated Films}

Figure 1 shows the experimental (dots) and fitted (solid lines) RBS data from monolithic and modulated $\mathrm{TiO}_{2}: \mathrm{Cr}, \mathrm{N}$ coatings. In this case, the monolithic film was produced with constant power $W_{\mathrm{Cr}}=15 \mathrm{~W}$ during the whole deposition time. The bilayer coating was produced with $W_{\mathrm{Cr}}=0 \mathrm{~W}$ for one half of the deposition time $(\sim 50 \mathrm{~nm})$, followed by switching on the discharge on the $\mathrm{Cr}$ cathode to $W_{\mathrm{Cr}}=15 \mathrm{~W}$ during the rest of the process. Finally, the gradient coating was produced with $W_{\mathrm{Cr}}=0 \mathrm{~W}$ for one fourth of the total deposition time $(\sim 25 \mathrm{~nm})$ and a stepwise increase of $W_{\mathrm{Cr}}$ by $5 \mathrm{~W}$ each $30 \mathrm{~min}$. The designed layer structures for the different coating architectures are depicted as inset in Figure 1. The contributions from the various elements to the overall RBS spectra are also labelled in the figure. The $\mathrm{Cr}$ contribution to the overall spectra obtained from the fitting results is also included in the graph and reflects the different layer arrangements. Moreover, the $\mathrm{Cr}$ doping level is similar $\left(\sim 4\right.$ at.\%) at the near surface region for all cases (where $W_{\mathrm{Cr}}=15 \mathrm{~W}$ ), with a $\mathrm{Cr} / \mathrm{Ti}$ ratio of around 0.15 . Obviously, the $\mathrm{Cr}$ concentration is constant for the whole film thickness in the monolithic film. The RBS cross-section is low for light elements and, hence, only the oxygen signal is clearly detected due to its large concentration. However, the $\mathrm{N}$ content is found to be around $2-3$ at. $\%$, as derived by complementary ERDA measurements.

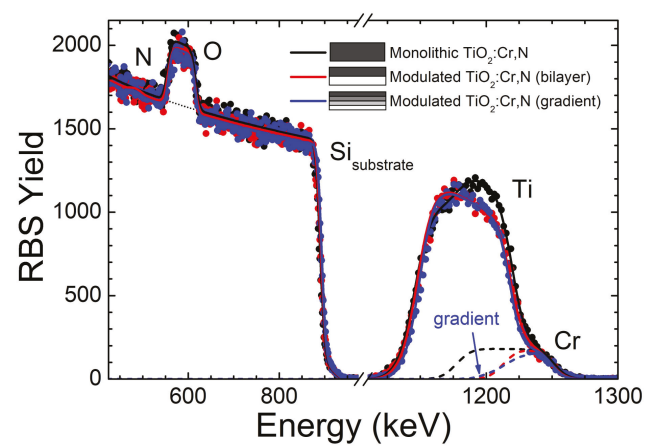

Figure 1. RBS (Rutherford backscattering spectrometry) data (dots) and fitted spectra (solid lines) for as-grown $\mathrm{TiO}_{2}: \mathrm{Cr}$, N coatings grown on $\mathrm{Si}(100)$ substrates with monolithic and modulated (bilayer and gradient architectures) structures. The individual element contribution from $\mathrm{Cr}$ to the fitted spectra is also shown (dotted lines). 


\subsection{Structural Investigations of Monolithic and Modulated Films}

Figure 2 shows the GI-XRD patterns from monolithic and modulated $\mathrm{TiO}_{2}: \mathrm{Cr}, \mathrm{N}$ coatings as well as from $\mathrm{TiO}_{2}$ and $\mathrm{TiO}_{2}: \mathrm{N}$ monolithic films grown under equivalent conditions. The $\mathrm{TiO}_{2}$ coating shows a mixture of rutile and anatase phases for this particular growth condition, although the relative phase contribution can be tuned, among other parameters, by the oxygen partial pressure [26]. Note that the broad bump around $55^{\circ}$ comes from the underlying $\mathrm{Si}(100)$ substrate. The dominant $\mathrm{A}_{101}$ reflection from the $\mathrm{TiO}_{2}: \mathrm{N}$ coating evidences that the addition of $\mathrm{N}$ under these conditions prevents rutile formation and yields a nearly single-phase anatase film. This effect was reported in detail in a previous publication by some of the present authors [20] and other groups [27-29]. In the Cr co-doped monolithic film, the structure displays a strong mixed-phase character where the rutile content is significantly higher than in the pure $\mathrm{TiO}_{2}$ film. As discussed in the introduction, this result supports the favourable formation of rutile environments upon $\mathrm{Cr}$ incorporation. The $\mathrm{R}_{110}$ reflection from rutile in the monolithic $\mathrm{TiO}_{2}: \mathrm{Cr}, \mathrm{N}$ coating is considerably broad, which can be attributed to both the structural disorder induced by $\mathrm{Cr}$ incorporation into the oxide matrix and, eventually, the formation of a rutile hybrid oxide in a similar fashion as observed in Cr-doped $\mathrm{TiO}_{2}$ [17]. Remarkably, the anatase selectivity in the $\mathrm{TiO}_{2}: \mathrm{N}$ buffer layer can be effectively used to imprint this structural phase to the co-doped layer in the modulated growth. Indeed, both modulated films display strong $\mathrm{A}_{101}$ Bragg reflections corresponding to a nearly single-phase structure. The similar result in both modulated designs supports the validity of the transfer process, where anatase keeps on growing by either increasing the $\mathrm{Cr}$ content progressively or abruptly. The widths of the $\mathrm{A}_{101}$ Bragg reflection indicate that the anatase phase is nanocrystalline, with grain sizes of around $20 \mathrm{~nm}$, as estimated by the Scherrer formula [30]. Finally, in all Cr-containing films, no evidence of $\mathrm{Cr}-\mathrm{O}$ phase segregation is observed.

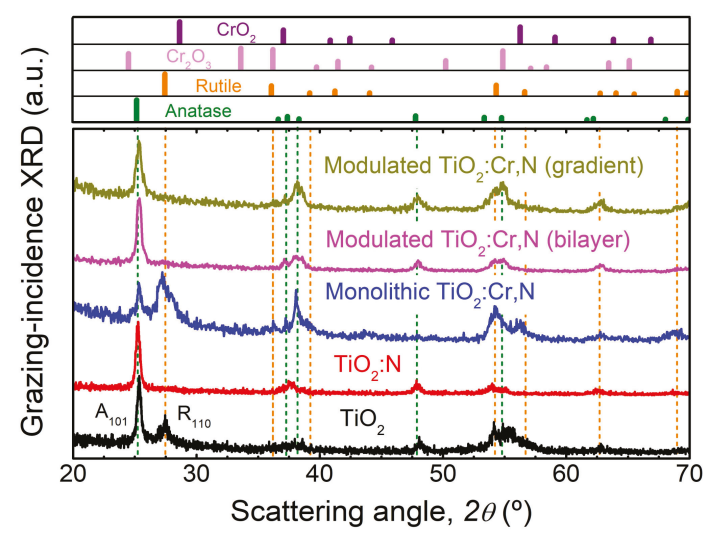

Figure 2. Grazing-incidence $X \mathrm{RD}$ patterns from $\mathrm{TiO}_{2}, \mathrm{TiO}_{2}: \mathrm{N}$ and $\mathrm{TiO}_{2}: \mathrm{Cr}, \mathrm{N}$ (monolithic/modulated) coatings grown under equivalent conditions. The diffraction patterns from anatase (PDF-00-021-1272), rutile (PDF-00-021-1276), $\mathrm{Cr}_{2} \mathrm{O}_{3}$ (PDF-00-001-0622) and $\mathrm{CrO}_{2}$ (PDF-00-001-0622) reference compounds are shown in the upper panel for phase identification.

For complementary phase identification at the near-surface region, the bonding structure around host and dopant sites has been studied in detail by XANES. The spectra for the Ti $2 p, \mathrm{O} 1 s, \mathrm{Cr} 2 p$ and $\mathrm{N} 1$ s element edges are shown in Figure 3. For the sake of clarity, only the spectra from the bilayer coating are shown as representative case of the modulated growth (same results were obtained from the gradient design). The element spectra from corresponding reference binary oxides as extracted from the literature are also shown for comparison. In particular, the $\mathrm{TiO}_{2}$ references refer to the spectra reported for polycrystalline films by Ruus et al. [31]. Regarding chromium oxides, we have included the 
spectra from single-crystal $\mathrm{Cr}_{2} \mathrm{O}_{3}$ [32], $\mathrm{CrO}_{2}$ deposited film [33] and $\mathrm{CrO}_{3}$ powder [34]. Accordingly, the spectral features from the latter oxides can be used as fingerprints of environments with oxidation state $\mathrm{Cr}^{3+}, \mathrm{Cr}^{4+}$ and $\mathrm{Cr}^{6+}$, respectively. Basically, the individual XANES spectra can be interpreted as a picture of the density of Ti-3d, O- $2 p, \mathrm{~N}-2 p$, and Cr-3d states according to dipole selection rules. A detailed description of the origin of the different spectral features and regions can be found in previous publications for binary $[26,35]$ and ternary $[17,20]$ oxides. The most direct information about the oxide matrix can be found in the Ti $2 p$ edge, where anatase and rutile $\mathrm{TiO}_{2}$ display clear distinct features, especially in the double-peak structure within the $459-462 \mathrm{eV}$ region. In this way, the Ti $2 p$ line shape indicates a dominant rutile and anatase structure for monolithic and modulated films, respectively. Then, surface-sensitive XANES results prove that the anatase phase in the $\mathrm{TiO}_{2}: \mathrm{N}$ buffer layer is clearly transferred from the interface up to the surface in the $\mathrm{Cr}$-containing layer by the modulated growth.

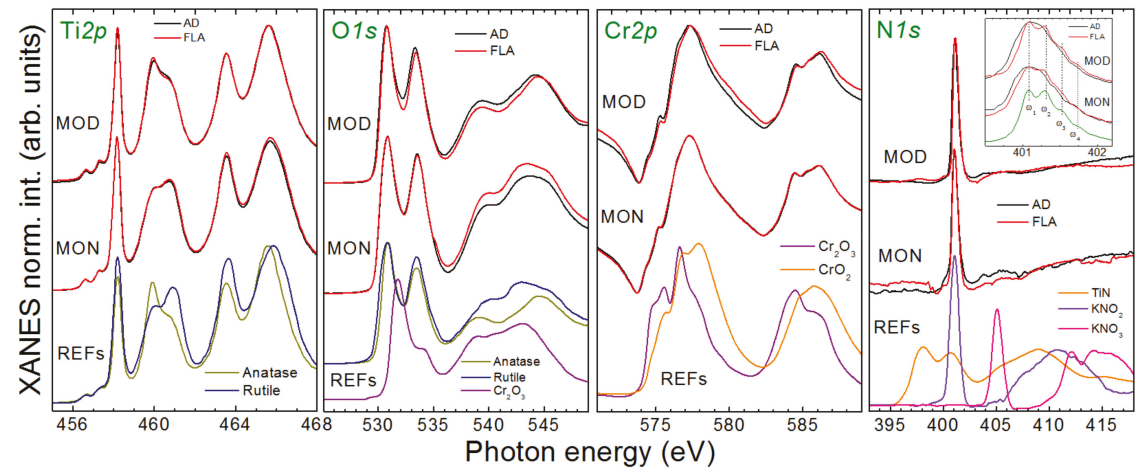

Figure 3. XANES spectra at the different element edges for monolithic (MON) and modulated (MOD) $\mathrm{TiO}_{2}: \mathrm{Cr}, \mathrm{N}$ coatings before (black) and after (red) FLA. The reference spectra from binary oxide compounds are also included in the bottom part (see text for details).

The $\mathrm{O} 1 s$ leads to similar conclusions in relation to the dominant titania phase. Moreover, there is no evidence of $\mathrm{O}-\mathrm{Cr}$ bonds, since the signal is dominated by the $\mathrm{TiO}_{2}$ matrix. In any case, information about the $\mathrm{Cr}$ sites can be extracted from the $\mathrm{Cr} 2 p$ edge. The $\mathrm{Cr} 2 p$ spectra are rather broad in comparison to the reference oxides due to the nanocrystalline nature of the films. The spectra show a marked background since the $\mathrm{Cr} 2 p$ edge is superimposed to the $\mathrm{O} 1$ s post-edge and, in addition, the $\mathrm{Cr}$ content in the films is relatively low. Here, the adsorption onset and spectral features indicate that most of the $\mathrm{Cr}$ dopants are in the $\mathrm{Cr}^{3+}$ oxidation state $[17,35]$. This situation has been considered as an indication of the substitutional nature of $\mathrm{Cr}$ dopants [6] and, from a practical point of view, can also be relevant to improve the photocatalytic response [10,11]. However, a certain contribution from $\mathrm{Cr}^{4+}\left(\mathrm{CrO}_{2}\right)$ may also be plausible by the increased intensity in the 578-579 and 586-588 eV regions. The presence of $\mathrm{Cr}^{4+}$ sites could also be correlated with the formation of a (secondary) mixed-oxide rutile phase [17], as suggested by the rather broad $\mathrm{R}_{110}$ Bragg reflection in the GI-XRD pattern from the monolithic $\mathrm{TiO}_{2}: \mathrm{Cr}, \mathrm{N}$ in Figure 2. Regarding $\mathrm{N}$ sites, the XANES N $1 s$ shows a main contribution of interstitial sites in the form of $-\mathrm{NO}_{x}$ radicals $(x \sim 2)$, as evidenced by the intense peak at $\sim 401 \mathrm{eV}$. However, as shown for the case of $\mathrm{N}$-doped $\mathrm{TiO}_{2}$ [26], such environments may have a relatively large cross-section and some $\mathrm{N}$ can also be in substitutional sites $(\mathrm{N}-\mathrm{Ti})$. This is further discussed below when presenting the XPS results.

\subsection{Effect of Flash-Lamp-Annealing (FLA)}

The impact of FLA on the structural properties of monolithic and modulated films was also examined. XANES spectra of the flashed (red curves) and as-deposited (black curves) films are 
compared in Figure 3. Since the films were grown at moderate temperatures, the modifications induced by FLA are not severe. However, there are subtle changes that are worth mentioning. First, the low energy peak at $\sim 461 \mathrm{eV}$ is promoted and sharpened at the $\mathrm{Ti} 2 p$ edge of the modulated film, which evidences a slight increase in the quality of the anatase phase. A similar structural improvement is hinted in the rutile features for the monolithic film. Another relevant change induced by FLA in both structures is related to the nature of $\mathrm{N}$ environments. In particular, the high-resolution scan of the $\mathrm{N} 1 \mathrm{~s}$ peak around $401 \mathrm{eV}$ (see inset) reveals the appearance of a fine structure, a fingerprint of the formation of $\mathrm{N}_{2}$ molecules inside the oxide matrix [20].

The structural changes induced by FLA were also studied by GI-XRD (note that this analysis was performed in smaller samples than those in Figure 2). In agreement with XANES, the XRD patterns in Figure 4 also show that the phase composition is not significantly altered by FLA. In this case, a small transformation into rutile takes place in the bilayer coating, which is not observed for the case of a gradient profile. In addition, the $\mathrm{A}_{101}$ Bragg peak in modulated coatings displays a shift after FLA to higher angles (smaller lattice parameter). This should be mainly attributed to strain effects, but another plausible contribution could be the promotion of $\mathrm{N}$ substitutional sites (see below).

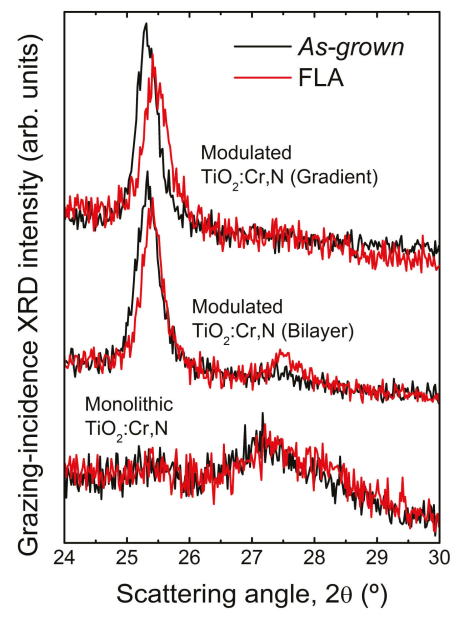

Figure 4. Grazing-incidence XRD patterns from monolithic and modulated $\mathrm{TiO}_{2}: \mathrm{Cr}, \mathrm{N}$ coatings before and after FLA. The anatase phase in modulated coatings shows a shift to higher scattering angles (lattice contraction) with the thermal treatment.

In order to deepen in the evolution of $\mathrm{N}$ environments, XPS was performed in as-deposited and FLA samples. The N 1s core-level spectra for the different samples are shown in Figure 5. In line with the XANES, XPS of as-grown samples shows the dominance of interstitial sites (N-O), together with the presence of $\mathrm{N}_{2}$ molecules $(\mathrm{N}-\mathrm{N})$. Note that FLA increases the relative contribution of $\mathrm{N}-\mathrm{N}$ bonds, as already pointed out by XANES. Remarkably, FLA also transforms N into substitutional sites (Ti-N) and, hence, it clearly favors activation of the anion dopant. 


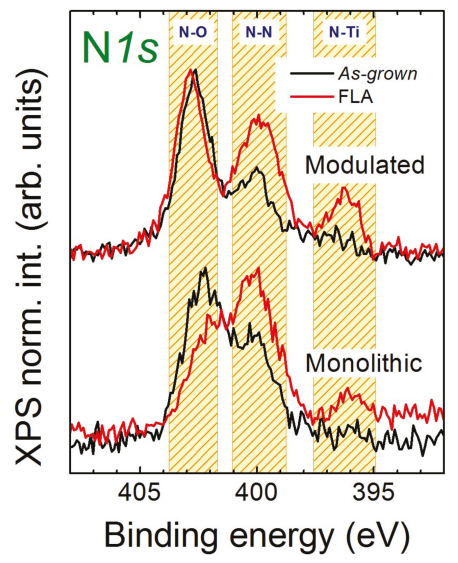

Figure 5. $\mathrm{N} 1 s$ core-level spectra from monolithic and modulated $\mathrm{TiO}_{2}: \mathrm{Cr}, \mathrm{N}$ coatings before and after FLA.

The impact of FLA on the film morphology and phase structure was also studied by TEM analysis. Figure 6 shows cross-sectional bright-field TEM images of the monolithic and modulated architecture with a gradient profile. In both samples, there is a thin $(\sim 2 \mathrm{~nm}) \mathrm{SiO}_{2}$ layer at the substrate-coating interface coming from the native oxide of the $\mathrm{Si}(100)$ wafers. The images show columnar grain growth with in-plane diameters of a few tens of nanometres, which is consistent with the grain size estimated by GI-XRD. Irrespective of the film architecture, the crystal growth is more or less homogenous across the whole layer thickness. The samples after FLA show a more pronounced grain orientation contrast close to the interface to the substrate, which can be correlated with an enhanced crystallinity in that region. The presence of pores in the early stage of growth can also be seen in both samples, although they are more evident in the $\mathrm{TiO}_{2}: \mathrm{N}$ buffer layer within the modulated design. Such pore structures can be resolved more clearly in slightly under-focused (UF) bright-field TEM images, as shown in Figure 6 from a magnified view of the film-substrate interface region. It is also relevant that those structures increase in size after FLA. The UF images also emphasize the enhancement (darker zones) of the film crystalline quality near the substrate interface after FLA. Finally, selected-area electron diffraction (SAED) analysis shown in Figure 6 from the imaged regions supports the phase formation derived from XANES and XRD, with a dominant rutile or anatase character for monolithic and modulated coatings, respectively.

Spectrum imaging analysis based on EDXS was done at the cross-sectional TEM specimens to study the in-depth element distributions. The corresponding HAADF-STEM micrographs and the EDXS element maps are shown in Figure 7. The images reveal homogenous distributions of Ti, O and $\mathrm{N}$ atoms. Note that there is also an additional narrow $\mathrm{N}$ contribution below the native oxide layer, which should be attributed to the commercial wafer fabrication process. The $\mathrm{Cr}$ distributions from the as-deposited samples clearly reproduce the coating designs, where the different interfaces resulting from the modulated growth can be identified, as highlighted by the dashed lines in the figure. The preservation of the expected profile also evidences that TEM specimen preparation has not altered the element distributions. Interestingly, the $\mathrm{Cr}$ distribution obtained after FLA in the modulated structure shows that the thermal treatment can (at least partially) homogenize the initial $\mathrm{Cr}$ profile. Therefore, one could design a modulated structure for phase selectivity and subsequently distribute the Cr dopants by FLA. Quantitative EDXS analysis (line scans not shown here) provide similar results as RBS and ERD with $\mathrm{Cr}$ and $\mathrm{N}$ contents of a few at.\%. In the monolithic film, there is a slight accumulation of $\mathrm{N}$ in the region where the aforementioned pores are observed (note the contrast in this region in the HAADF-STEM images due to the reduced atomic number). Therefore, we 
assume that such mesoporous structure may be filled with $\mathrm{N}_{2}$ (bubbles). Note that the promotion of $\mathrm{N}_{2}$ after FLA has been detected at the near-surface region by XANES and XPS. In this regard, it should be mentioned that the reduced $\mathrm{N}$ signal in the pore region for the FLA-treated modulated film (see Figure 7) is most probably caused by $\mathrm{N}_{2}$ release from the enlarged and interconnected pores, which get opened during preparation of the thin electron-transparent TEM lamella.
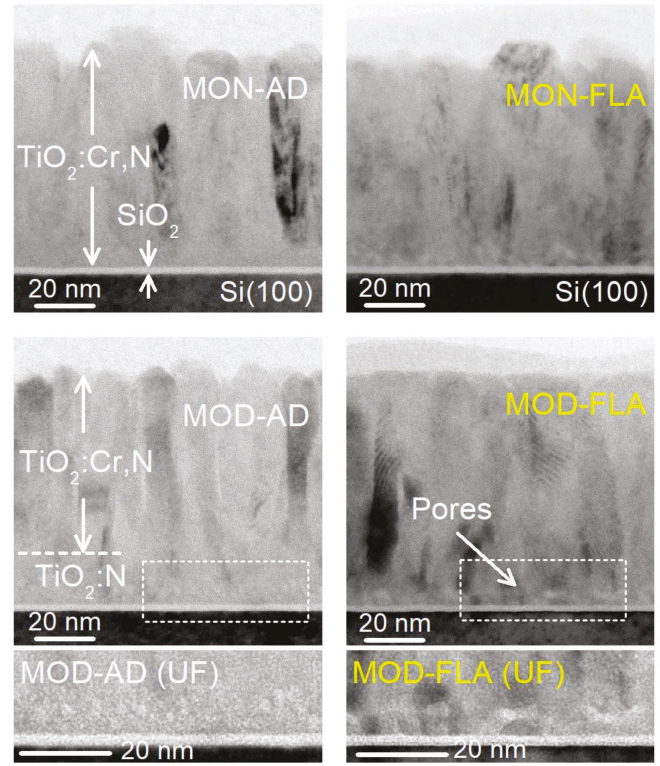

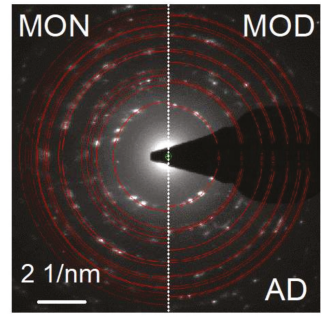

(a)

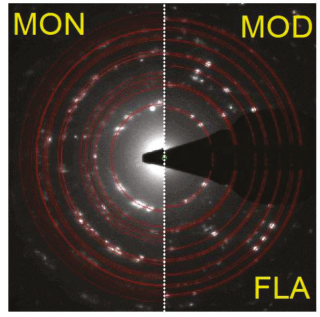

(b)

Figure 6. Cross-sectional bright-field TEM images from monolithic (MON) and modulated (MOD) $\mathrm{TiO}_{2}: \mathrm{Cr}$,N coatings as-deposited (AD) (a) and after FLA (b). The slightly under-focused (UF) bright-field images in the third row are magnified views of the marked regions close to the substrate-coating interface in the MOD case to enhance the contrast of the pore structures. Corresponding representative SAED patterns displayed in the bottom part confirm the dominant rutile and anatase character for MON and MOD coatings, respectively. 


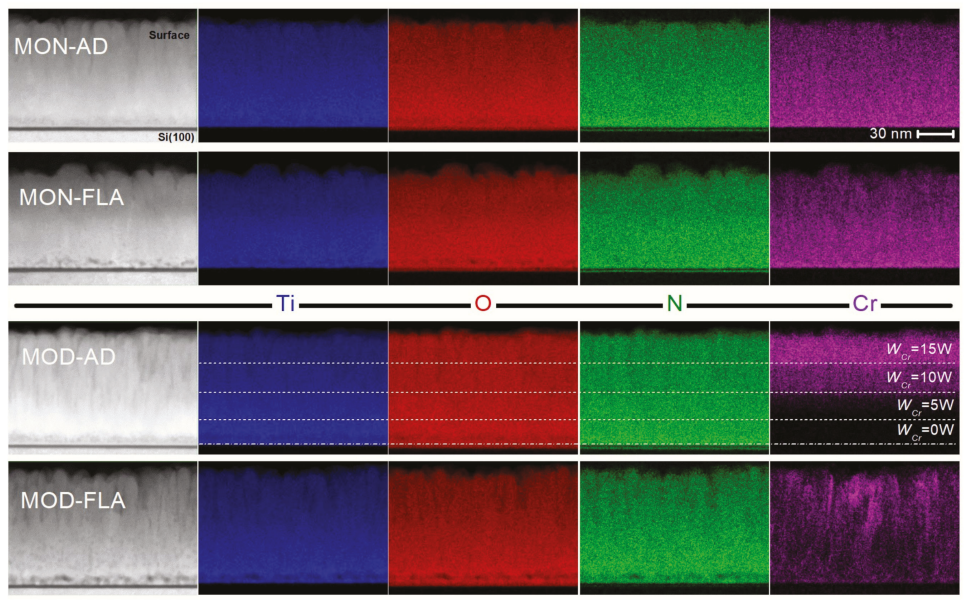

Figure 7. HAADF-STEM micrographs and element distributions obtained by EDXS analysis of monolithic (MON) and modulated (MOD) coatings in as-deposited (AD) and after FLA states. Dashed lines in the MOD-AD coating indicate the interfaces caused by the gradient steps during film growth.

\subsection{Final Remarks}

One relevant question regarding the modulated growth relies on the generalization of the phase selectivity for other growth conditions and coating designs. For this purpose, additional processing windows were tested. First, higher $W_{\mathrm{Cr}}$ were sampled for the stability of the anatase phase against higher $\mathrm{Cr}$ contents in the films. In particular, bilayer films were produced with $W_{\mathrm{Cr}}$ up to $25 \mathrm{~W}$ in the second growth stage. This change implies a slight increase of the $\mathrm{Cr}$ content from $\sim 4$ to $\sim 5$ at. $\%$, as extracted from RBS. As shown in the Ti $2 p$ XANES spectra in Figure 8, the progressive increase in $\mathrm{Cr}$ results in a higher promotion of the rutile phase. Hence, single-phase anatase $\mathrm{TiO}_{2}: \mathrm{Cr}, \mathrm{N}$ layers produced in modulated structures can only accommodate $\mathrm{Cr}$ contents below the threshold of $\sim 5$ at. $\%$. Interestingly, the Ti $2 p$ spectra from the samples after FLA (red curves) confirm that the thermal treatment improves the crystal structure, and even restores the anatase dominance for the highest $\mathrm{Cr}$ contents. The latter could be related to the aforementioned redistribution of $\mathrm{Cr}$ after FLA, which would imply an effective decrease in the Cr content. Such a hypothesis is supported by the relative intensity of the corresponding XANES edges (not shown).

Another crucial parameter in the modulated growth is the substrate temperature. As indicated in the experimental section, we selected the lowest temperature $\left(300^{\circ} \mathrm{C}\right)$ capable of producing high-quality anatase $\mathrm{TiO}_{2}: \mathrm{N}$ layer [20] for the buffer layer. This means that we could obtain better (worse) quality anatase phase at higher (lower) temperatures. The thickness of the Cr-containing layer should also be considered to test how long the anatase growth can be sustained in the modulated mode. To study this influence, we prepared bilayer coatings by increasing the Cr-containing layer from 50 to $75 \mathrm{~nm}$. In the latter case, the structure preserves the anatase character, but the rutile contribution starts to increase for the thicker layer. 


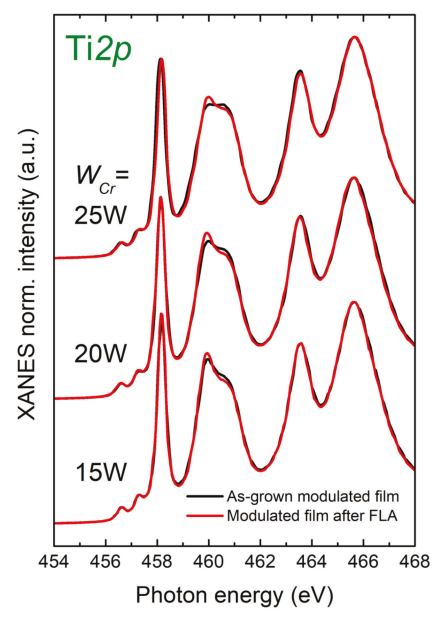

Figure 8. XANES spectra of modulated films with a bilayer structure produced with different $W_{\mathrm{Cr}}$ in the uppermost layer in as-grown (black curves) and after FLA (red curves) states.

Finally, in the present work, we have focused our investigations on the structural properties related to the modulated growth. Obviously, additional efforts should be devoted to varying the FLA conditions and modulated designs in order to optimize the structural quality. In addition, a natural continuation of this work would be testing the performance of these coatings for photocatalytic applications.

\section{Conclusions}

In conclusion, we have studied the phase formation in $\mathrm{TiO}_{2}: \mathrm{Cr}, \mathrm{N}$ thin films and exploited interface engineering concepts for phase selectivity. In particular, monolithic coatings result in the formation of a mixed-phase oxide with dominant rutile character. Remarkably, under equivalent conditions, the favourable formation of single-phase anatase in a $\mathrm{TiO}_{2}: \mathrm{N}$ buffer layer can be utilized to transfer this structure into $\mathrm{TiO}_{2}: \mathrm{Cr}, \mathrm{N}$. Such phase selectivity in the $\mathrm{Cr}$-containing film occurs in both gradual and abrupt $\mathrm{Cr}$ profiles. However, the production of single-phase anatase seems to be limited to low $\mathrm{Cr}$ contents $(<5$ at.\%) and is less effective as the film thickness is increased. Post-deposition millisecond-range FLA was used to enhance the quality of the as-grown phase together with dopant activation and diffusion. This work shows that the combination of modulated growth designs and FLA opens new opportunities for tailoring the desired structure of $\mathrm{TiO}_{2}$-based materials.

Author Contributions: Conceptualization, R.G. and S.P.; Methodology, R.G. and S.P.; Validation, R.G., S.P., and R.H.; Formal Analysis, R.G., S.P., R.H. and F.M.; Investigation, R.G., S.P., R.H., F.M., D.E.-M., I.J. and J.P.; Resources, R.G., S.P., R.H., F.M. and J.P.; Writing-Original Draft Preparation, R.G.; Writing-Review and Editing, R.G., S.P., R.H., F.M., D.E.-M., I.J. and J.P.; Visualization, R.G. and R.H.; Supervision, R.G. and S.P.; Project Administration, R.G. and S.P.; Funding Acquisition, R.G., S.P., R.H., I.J., and J.P.

Funding: The research leading to these results has received funding from projects RTI2018-095137-B-I00 and MAT2016-80394-R from Ministerio de Ciencia, Innovación y Universidades (Spain) and grant agreement $n^{\circ} 226716$ from the European Community's Seventh Framework Programme (FP7/2007-2013). Funding of TEM Talos F200X by the German Federal Ministry of Education of Research (BMBF) through Grant No. 03SF0451 in the framework of HEMCP is gratefully acknowledged.

Acknowledgments: We thank HZB and HZDR for the allocation of beamtime for the synchrotron and ion beam analysis experiments, respectively. Support by the TEM facilities at IBC-HZDR is gratefully acknowledged. The authors would also like to thank R. Aniol at HZDR for TEM specimen preparation.

Conflicts of Interest: The authors declare no conflict of interest. 


\section{References}

1. Diebold, U. The surface science of titanium dioxide. Surf. Sci. Rep. 2003, 48, 53-229. [CrossRef]

2. Henderson, M.A. A surface science perspective on $\mathrm{TiO}_{2}$ photocatalysis. Surf. Sci. Rep. 2011, 66, 185-297. [CrossRef]

3. Matsumoto, Y.; Murakami, M.; Shono, T.; Hasegawa, T.; Fukumura, T.; Kawasaki, M.; Ahmet, P.; Chikyow, T.; Koshihara, S.; Koinuma, H. Room-temperature ferromagnetism in transparent transition metal-doped titanium dioxide. Science 2001, 291, 854-856. [CrossRef] [PubMed]

4. Serpone, N. Is the band gap of pristine $\mathrm{TiO}_{2}$ narrowed by anion- and cation-doping of titanium dioxide in second-generation photocatalysts? J. Phys. Chem. B 2006, 110, 24287-24293. [CrossRef] [PubMed]

5. Vitiello, G.; Pezzella, A.; Calcagno, V.; Silvestri, B.; Raiola, L.; D’Errico, G.; Costantini, A.; Branda, F.; Luciani, G. 5,6-Dihydroxyindole-2-carboxylic acid- $\mathrm{TiO}_{2}$ charge transfer complexes in the radical polymerization of melanogenic precursor(s). J. Phys. Chem. C 2016, 120, 6262-6268. [CrossRef]

6. Chiodi, M.; Cheney, C.P.; Vilmercati, P.; Cavaliere, E.; Mannella, N.; Weitering, H.H.; Gavioli, L. Enhanced dopant solubility and visible-light absorption in $\mathrm{Cr}-\mathrm{N}$ codoped $\mathrm{TiO}_{2}$ nanoclusters. J. Phys. Chem. C 2012, 116, 311-318. [CrossRef]

7. Asahi, R.; Morikawa, T.; Ohwaki, T.; Aoki, K.; Taga, Y. Visible-light photocatalysis in nitrogen-doped titanium oxides. Science 2001, 293, 269-271. [CrossRef]

8. Batzill, M.; Morales, E.H.; Diebold, U. Influence of nitrogen doping on the defect formation and surface properties of $\mathrm{TiO}_{2}$ rutile and anatase. Phys. Rev. Lett. 2006, 96, 026103. [CrossRef]

9. Clarizia, L.; Vitiello, G.; Pallotti, D.K.; Silvestri, B.; Nadagouda, M.; Lettieri, S.; Luciani, G.; Andreozzi, R.; Maddalena, P.; Marotta, R. Effect of surface properties of copper-modified commercial titanium dioxide photocatalysts on hydrogen production through photoreforming of alcohols. Int. J. Hydrogen Energy 2017, 42, 28349-28362. [CrossRef]

10. Zhu, J.; Deng, Z.; Chen, F.; Zhang, J.; Chen, H.; Anpo, M.; Huang, J.; Zhang, L. Hydrothermal doping method for preparation of $\mathrm{Cr}^{3+}-\mathrm{TiO}_{2}$ photocatalysts with concentration gradient distribution of $\mathrm{Cr}^{3+}$. Appl. Catal. B: Environ. 2006, 62, 329-335. [CrossRef]

11. Fan, X.; Chen, X.; Zhu, S.; Li, Z.; Yu, T.; Ye, J.; Zou, Z. The structural, physical and photocatalytic properties of the mesoporous Cr-doped $\mathrm{TiO}_{2}$. J. Mol. Catal. A Chem. 2008, 284, 155-160. [CrossRef]

12. Kaspar, T.C.; Heald, S.M.; Wang, C.M.; Bryan, J.D.; Droubay, T.; Shutthanandan, V.; Thevuthasan, S.; McCready, D.E.; Kellock, A.J.; Gamelin, D.R.; et al. Negligible magnetism in excellent structural quality $\mathrm{Cr}_{x} \mathrm{Ti}_{1-x} \mathrm{O}_{2}$ anatase: contrast with high- $T_{\mathrm{C}}$ ferromagnetism in structurally defective $\mathrm{Cr}_{x} \mathrm{Ti}_{1-x} \mathrm{O}_{2}$. Phys. Rev . Lett. 2005, 95, 217203. [CrossRef] [PubMed]

13. Zhu, W.; Qiu, X.; Iancu, V.; Chen, X.Q.; Pan, H.; Wang, W.; Dimitrijevic, M.N.; Rajh, T.; Meyer, H.M.; Paranthaman, M.P.; et al. Band gap narrowing of titanium oxide semiconductors by noncompensated anion-cation codoping for enhanced visible-light photoactivity. Phys. Rev. Lett. 2009, 103, 226401. [CrossRef] [PubMed]

14. Wang, Y.; Cheng, Z.; Tan, S.; Shao, X.; Wang, B.; Hou, J.G. Characterization of Cr-N codoped anatase $\mathrm{TiO}_{2}(001)$ thin films epitaxially grown on $\mathrm{SrTiO}_{3}(001)$ substrate. Surf. Sci. 2013, 616, 93. [CrossRef]

15. Cheng, Z.; Zhang, L.; Dong, S.; Ma, X.; Ju, H.; Zhu, J.; Cui, X.; Zhao, J.; Wang, B. Electronic properties of Cr-N codoped rutile $\mathrm{TiO}_{2}(110)$ thin films. Surf. Sci. 2017, 666, 84-89. [CrossRef]

16. Kollbek, K.; Szkudlarek, A.; Marzec, M.M.; Lyson-Sypien, B.; Cecot, M.; Bernasik, A.; Radecka, M.; Zakrzewska, K. Optical and electrical properties of $\mathrm{Ti}(\mathrm{Cr}) \mathrm{O}_{2}: \mathrm{N}$ thin films deposited by magnetron co-sputtering. Appl. Surf. Sci. 2016, 380, 73-82. [CrossRef]

17. Gago, R.; Prucnal, S.; Pérez-Casero, R.; Caretti, I.; Jiménez, I.; Lungwitz, F.; Cornelius, S. Structural impact of chromium incorporation in as-grown and flash-lamp-annealed sputter deposited titanium oxide films. J. Alloy Compd. 2017, 729, 438-445. [CrossRef]

18. Xu, M.; Gao, Y.; Martinez-Moreno, E.; Kunst, M.; Muhler, M.; Wang, Y.; Idriss, H.; Wöll, C. Photocatalytic activity of bulk $\mathrm{TiO}_{2}$ anatase and rutile single crystals using infrared absorption spectroscopy. Phys. Rev. Lett. 2011, 106, 138302. [CrossRef]

19. Houska, J.; Mraz, S.; Schneider, J.M. Experimental and molecular dynamics study of the growth of crystalline $\mathrm{TiO}_{2}$. J. Appl. Phys. 2012, 112, 073527. [CrossRef] 
20. Gago, R.; Redondo-Cubero, A.; Vinnichenko, M.; Lehmann, J.; Munnik, F.; Palomares, F.J. Spectroscopic evidence of $\mathrm{NO}_{x}$ formation and band-gap narrowing in $\mathrm{N}$-doped $\mathrm{TiO}_{2}$ films grown by pulsed magnetron sputtering. Mater. Chem. Phys. 2012, 136, 729-736. [CrossRef]

21. Skorupa, W.; Gebel, T.; Yankov, R.A.; Paul, S.; Lerch, W.; Downey, D.F.; Arevalo, E.A. Advanced thermal processing of ultrashallow implanted junctions using flash lamp annealing. J. Electrochem. Soc. 2005, 152, G436-G440. [CrossRef]

22. Mayer, M. SIMNRA User's Guide 6.05; Max-Planck-Institut für Plasmaphysik: Garching, Germany, 2009.

23. Barradas, N.P.; Jeynes, C.; Webb, R.P. Simulated annealing analysis of Rutherford backscattering data. Appl. Phys. Lett. 1997, 71, 291-293. [CrossRef]

24. Stöhr, J. NEXAFS Spectroscopy; Springer: New York, NY, USA, 1992.

25. Crocombette, J.P.; Jollet, F. Ti $2 p$ X-ray absorption in titanium dioxides $\left(\mathrm{TiO}_{2}\right)$ : The influence of the cation site environment. J. Phys. Condens. Matter 1994, 6, 10811. [CrossRef]

26. Gago, R.; Vinnichenko, M.; Redondo-Cubero, A.; Czigány, Z.; Vázquez, L. Surface morphology of heterogeneous nanocrystalline rutile/amorphous anatase $\mathrm{TiO}_{2}$ films grown by reactive pulsed magnetron sputtering. Plasma Process. Polym. 2010, 7, 813-823. [CrossRef]

27. Lindgren, T.; Mwabora, J.M.; Avendano, E.; Jonsson, J.; Hoel, A.; Granqvist, C.G.; Lindquist, S. Photoelectrochemical and optical properties of nitrogen doped titanium dioxide films prepared by reactive DC magnetron sputtering. J. Phys. Chem. B 2003, 107, 5709-5716. [CrossRef]

28. Madhavi, V.; Kondaiah, P.; Mohan Rao, G. Influence of silver nanoparticles on titanium oxide and nitrogen doped titanium oxide thin films for sun light photocatalysis. Appl. Surf. Sci. 2018, 436, 708-719.

29. Mwabora, J.M.; Lindgren, T.; Avendaño, E.; Jaramillo, T.F.; Lu, J.; Lindquist, S.E.; Granqvist, C.G. Structure, composition, and morphology of photoelectrochemically active $\mathrm{TiO}_{2-x} \mathrm{~N}_{x}$ thin films deposited by reactive DC magnetron sputtering. J. Phys. Chem. B 2004, 108, 20193-20198. [CrossRef]

30. Klug, H.P.; Alexander, L.E. X-ray Diffraction Procedures for Polycrystalline and Amorphous Materials; Wiley: Hoboken, NJ, USA, 1974.

31. Ruus, R.; Kikas, A.; Saar, A.; Ausmees, A.; Nommiste, E.; Aarik, J.; Aidla, A.; Uustare, T.; Martinson, I. Ti 2p and $\mathrm{O} 1 s \mathrm{X}$-ray absorption of $\mathrm{TiO}_{2}$ polymorphs. Solid State Commun. 1997, 104, 199-203. [CrossRef]

32. Kucheyev, S.O.; Sadigh, B.; Baumann, T.F.; Wang, Y.M.; Felter, T.E.; Van Buuren, T.; Gash, A.E.; Satcher, J.H., Jr.; Hamza, A.V. Electronic structure of chromia aerogels from soft X-ray absorption spectroscopy. J. Appl. Phys. 2007, 101, 124315. [CrossRef]

33. Schedel-Niedrig, T.; Neisius, T.; Simmons, C.T.; Köhler, K. X-ray absorption spectroscopy of small chromium oxide particles $\left(\mathrm{Cr}_{2} \mathrm{O}_{3}, \mathrm{CrO}_{2}\right)$ supported on titanium dioxide. Langmuir 1996, 12, 6377-6381.

34. Dedkov, Y.S.; Vinogradov, A.S.; Fonin, M.; König, C.; Vyalikh, D.V.; Preobrajenski, A.B.; Krasnikov, S.A.; Kleimenov, E.Y.; Nesterov, M.A.; Rüdiger, U.; et al. Correlations in the electronic structure of half-metallic ferromagnetic $\mathrm{CrO}_{2}$ films: An X-ray absorption and resonant photoemission spectroscopy study. Phys. Rev. B 2005, 72, 060401(R). [CrossRef]

35. Gago, R.; Vinnichenko, M.; Hübner, R.; Redondo-Cubero, A. Bonding structure and morphology of chromium oxide films grown by pulsed-DC reactive magnetron sputter deposition. J. Alloy Compd. 2016, 672, 529-535. [CrossRef]

(C) 2019 by the authors. Licensee MDPI, Basel, Switzerland. This article is an open access article distributed under the terms and conditions of the Creative Commons Attribution (CC BY) license (http://creativecommons.org/licenses/by/4.0/). 

Article

\title{
The Effect of RF Sputtering Conditions on the Physical Characteristics of Deposited GeGaN Thin Film
}

\author{
Cao Phuong Thao ${ }^{1}$, Dong-Hau Kuo ${ }^{2, *}$, Thi Tran Anh Tuan ${ }^{3, *}$, Kim Anh Tuan ${ }^{1}$, \\ Nguyen Hoang $\mathrm{Vu}^{1}$, Thach Thi Via Sa $\mathrm{Na}^{1}$, Khau Van Nhut ${ }^{1}$ and Nguyen Van Sau ${ }^{3}$ \\ 1 School of Engineering and Technology, Tra Vinh University, Tra Vinh 87000, Vietnam; \\ cpthao@tvn.edu.cn (C.P.T.); katuan@tvu.edu.cn (K.A.T.); nghvu@tvu.edu.vn (N.H.V.); \\ viasana@tvu.edu.cn (T.T.V.S.N.); nhutkhau@tvu.edu.vn (K.V.N.) \\ 2 Department of Materials Science and Engineering, National Taiwan University of Science and Technology, \\ Taipei 10607, Taiwan \\ 3 School of Basic Sciences, Tra Vinh University, Tra Vinh 87000, Vietnam; nvsau@tvu.edu.vn \\ * Correspondence: dhkuo@mail.ntust.edu.tw (D.-H.K.); thitrananhtuan@tvu.edu.vn (T.T.A.T.); \\ Tel.: +886-2-27303291 (D.-H.K.)
}

Received: 5 September 2019; Accepted: 1 October 2019; Published: 6 October 2019

\begin{abstract}
Ge}_{0.07} \mathrm{GaN}$ films were successfully made on $\mathrm{Si}(100), \mathrm{SiO}_{2} / \mathrm{Si}(100)$ substrates by a radio frequency reactive sputtering technique at various deposition conditions listed as a range of $100-400^{\circ} \mathrm{C}$ and $90-150 \mathrm{~W}$ with a single ceramic target containing 7 at \% dopant Ge. The results showed that different RF sputtering power and heating temperature conditions affected the structural, electrical and optical properties of the sputtered $\mathrm{Ge}_{0.07} \mathrm{GaN}$ films. The as-deposited $\mathrm{Ge}_{0.07} \mathrm{GaN}$ films had an structural polycrystalline. The GeGaN films had a distorted structure under different growth conditions. The deposited- $150 \mathrm{~W} \mathrm{Ge} 0.07 \mathrm{GaN}$ film exhibited the lowest photoenergy of $2.96 \mathrm{eV}$, the highest electron concentration of $5.50 \times 10^{19} \mathrm{~cm}^{-3}$, a carrier conductivity of $35.2 \mathrm{~S} \cdot \mathrm{cm}^{-1}$ and mobility of $4 \mathrm{~cm}^{2} \cdot \mathrm{V}^{-1} \cdot \mathrm{s}^{-1}$.
\end{abstract}

Keywords: Ge donor; GaN; growth condition; heating substrate temperature; RF power; reactive sputtering; thin film property

\section{Introduction}

It is known that Gallium Nitride (GaN) and its compounds have wide bandgap, high thermal conductivity [1] and wurtzite crystal structure. They have been employed for electronics and photo-electronic components, listed as MOSFET and HJ-FET transistors, diodes and light emitting diodes (LED) [2-6].

By using the doping technique to make n-type semiconductor materials, Shuji et al. studied the efficiency between $\mathrm{Ge}$ and $\mathrm{Si}$ doping. The doping of $\mathrm{Si}$ had higher efficiency as the $\mathrm{GeH}_{4}$ and $\mathrm{SiH}_{4}$ precursors were applied for $\mathrm{Ge}$ - and Si-doped $\mathrm{GaN}$ with high electron concentrations at $2 \times$ $10^{19}$ and $1 \times 10^{19} \mathrm{~cm}^{-3}$, respectively [7]. Ge performed as a charge carrier in GaN film made by a plasma-assisted molecular beam epitaxy (MBE) system [8,9]. Many researchers have applied various deposition techniques to make Ge-doped GaN, such as hydride-vapor phase-epitaxy (HVPE) [10], chemical-vapor-deposition (MOCVD) [7,11,12], metalorganic -vapor- phase-epitaxy (MOVPE) [13,14], and a thermionic-vacuum arc [15].

To investigate the influences of dopant on the semiconductor behaviors, in our previous experiment, we reported Ge-doped GaN film deposited by a radio frequency (RF) reactive sputtering technique with a single ceramic target at the different Ge contents of the dopant of 0, 0.03, 0.07 and 1 . It was 
presented that all these Ge-doped GaN thin films acted in as an n-type semiconductor for the various Ge dopant ratios [16]. Besides, there were many previous works that studied the effects of different sputtering conditions on the doping GaN films $[5,17,18]$. However, there is not much research exploring the influence of the different growth conditions on Ge-doped GaN film made by the RF reactive sputtering technique until this work. In this research, we study the effects of RF sputtering conditions on properties of these $\mathrm{Ge}_{0.07} \mathrm{GaN}$ films. Firstly, $\mathrm{Ge}_{0.07} \mathrm{GaN}$ films were grown at different heating substrate temperatures from 100 to $400{ }^{\circ} \mathrm{C}$. Secondly, the RF sputtering power changed in the range of $90-150 \mathrm{~W}$ and was applied to prepare $\mathrm{Ge}_{0.07} \mathrm{GaN}$ films, while the deposition temperature was fixed at $300{ }^{\circ} \mathrm{C}$.

\section{Experimental Details}

$\mathrm{Ge}_{0.07} \mathrm{GaN}$ thin films were successfully deposited on $\mathrm{Si}(100)$ substrate by radio-frequency (RF) reactive sputtering with a $\mathrm{Ge}_{0.07} \mathrm{GaN}$ single ceramic target containing 7 at the $\%$ of the $\mathrm{Ge} /(\mathrm{Ge}+\mathrm{Ga})$ molar ratio. To investigate the influences of deposition temperature, the substrates were heated in a range of $100-400{ }^{\circ} \mathrm{C}$ while the output $\mathrm{RF}$ power and sputtering time were kept at $120 \mathrm{~W}$ and $30 \mathrm{~min}$, respectively. To study the effects of different sputtering powers on properties of $\mathrm{Ge}_{0.07} \mathrm{GaN}$ films, the films were deposited under 90,120, and $150 \mathrm{~W}$ while the deposition temperature and duration of sputtering were held at $300{ }^{\circ} \mathrm{C}$ and $30 \mathrm{~min}$, respectively. The sputtering proceeded under the working pressure at $9 \times 10^{-3}$ torrs and the mixing gases of Argon flow rate at $5 \mathrm{sccm}$ and Nitrogen flow rate at $15 \mathrm{sccm}$. The size of the single cermet targets employed in RF sputtering was $5.08 \mathrm{~cm}$ (2 inches). The distance between the target and substrates in the working chamber for depositing was kept at $5 \mathrm{~cm}$, while the substrate faced the target. Details for preparing a single ceramic target and RF reactive sputtering process were presented in the previous experiment in our laboratory $[16,18-21]$.

The structural crystallite of the sputtered $\mathrm{Ge}_{0.07} \mathrm{GaN}$ films deposited under the different heating substrates (range of $100-400^{\circ} \mathrm{C}$ ) was tested by X-ray diffractometry (XRD, D8 Discover, Bruker, Billerica, MA, USA). The morphological and topographical surfaces of these $\mathrm{Ge}_{0.07} \mathrm{GaN}$ films were investigated by scanning electron microscopy (SEM, JSM-6500F, JEOL, Tokyo, Japan) and atomic force microscopy (AFM, Dimension Icon, Bruker). The energy dispersive spectrometer (EDS, JSM-6500F, JEOL) prepared on SEM was employed to analyze the composition data of these films. A Hall measurement system (HMS-2000, Ecopia, Tokyo, Japan) including a maximum magnetic-field of 0.51T was applied for electrical properties. An Ultraviolet-Visible (UV-Vis) spectrometer (V-670, Jasco, Tokyo, Japan) was used to study the optical properties of $\mathrm{Ge}_{0.07} \mathrm{GaN}$ films.

\section{Results and Discussion}

\subsection{Effects of Growth Temperature on the Sputtered GeGaN Film Properties}

Compositional EDS investigation of the $\mathrm{Ge}_{0.07} \mathrm{GaN}$ films deposited in a temperature range from 100 to $400{ }^{\circ} \mathrm{C}$ is shown in Table 1. It is shown that the grown $\mathrm{Ge}_{0.07} \mathrm{GaN}$ films contained nitrogen from $48.4-49.7$ at.\%, and the $[\mathrm{N}] /([\mathrm{Ga}]+[\mathrm{Ge}])$ molar ratios were between $0.93-0.98$. It was illustrated that these $\mathrm{Ge}_{0.07} \mathrm{GaN}$ films were composed of slightly deficient nitrogen contents, and inadequate nitrogen was associated with the electrical properties of films. From EDS data displayed in Table 1, $[\mathrm{Ge}] /([\mathrm{Ge}]+[\mathrm{Ga}])$ molar ratios were 0.057, 0.074, 0.085, and 0.094 for Ge-0.07-GaN films at heating substrate temperatures of $100,200,300$ and $400{ }^{\circ} \mathrm{C}$, respectively. As the heating substrate temperature increased, there was an increase in the Ge molar ratios of the sputtered $\mathrm{Ge}_{0.07} \mathrm{GaN}$ films. It was indicated that deposition temperature changed the Ge atom ratio in the deposited film to prove the effect of sputtering temperature on the film properties.

The morphological and topographical surface images of $\mathrm{Ge}_{0.07} \mathrm{GaN}$ films deposited at different deposition temperatures in the range from 100 to $400{ }^{\circ} \mathrm{C}$ are displayed in Figure 1. The SEM surface images indicated that the grown $\mathrm{Ge}_{0.07} \mathrm{GaN}$ films had a microstructure with continuous and smooth surfaces. From the cross-sectional SEM patterns in Figure 1, these $\mathrm{Ge}_{0.07} \mathrm{GaN}$ films had a 1.0-1.78 $\mu \mathrm{m}$ 
thickness and adhered well between $\mathrm{Ge}_{0.07} \mathrm{GaN}$ films and $\mathrm{Si}$ wafer with free cracks or voids at interfaces. From data seen in Table 2, as the growth temperature rose from 100 to $400{ }^{\circ} \mathrm{C}$, the growth rate corresponded to $33.33,39.0,43.33$, and $59.33 \mathrm{~nm} / \mathrm{min}$. The root-mean-square (rms) roughness values of these deposited $\mathrm{Ge}_{0.07} \mathrm{GaN}$ films were 1.35, 1.40, 3.0, $3.1 \mathrm{~nm}$ as the substrate temperatures in the sputtering process increased from 100 to $400{ }^{\circ} \mathrm{C}$. The sputtered GaN film made by RF sputtering technology had a roughness from 0.7 to $20 \mathrm{~nm}$ [22], while the roughness of the GaN films made by the MOCVD method was in the range of $0.5-3 \mathrm{~nm}$ [23]. As the deposition temperature changed from 100 to $400{ }^{\circ} \mathrm{C}, \mathrm{Ge}_{0.07} \mathrm{GaN}$ films deposited had an increase in roughness value from 1.35 to $3.1 \mathrm{~nm}$ and a smooth surface. The morphology of the $\mathrm{Ge}_{0.07} \mathrm{GaN}$ film became rougher as the heating temperature substrate increased. It could be determined that strong bombardment of argon against the $\mathrm{Ge}_{0.07} \mathrm{GaN}$ target at a higher RF sputtering temperature was responsible for the faster deposition rate and the higher roughness of the surface.

Table 1. EDS composition of $\mathrm{Ge}_{0.07} \mathrm{GaN}$ films under different deposition conditions.

\begin{tabular}{ccccccc}
\hline Sputtering Conditions & Ga (at. $\%)$ & Ge (at. $\%)$ & N (at. $\%)$ & \multicolumn{1}{c}{ [Ge]/([Ga]+[GeIN]/([Ga]+[Ge]) } \\
\hline Heating & 100 & 47.42 & 2.87 & 49.71 & 0.057 & 0.988 \\
Substrate $\left({ }^{\circ} \mathrm{C}\right)$ & 200 & 47.08 & 3.78 & 49.14 & 0.074 & 0.966 \\
& 300 & 46.83 & 4.35 & 48.82 & 0.085 & 0.954 \\
\hline \multirow{2}{*}{ RF Sputtering } & 400 & 46.73 & 4.87 & 48.40 & 0.094 & 0.938 \\
Power (W) & 120 & 49.39 & 3.89 & 46.72 & 0.073 & 0.877 \\
& 150 & 46.83 & 4.35 & 48.82 & 0.085 & 0.954 \\
\hline
\end{tabular}

Table 2. The influence of RF power and substrate temperature conditions on the structural properties.

\begin{tabular}{ccccc}
\hline \multicolumn{2}{c}{ Sputtering Conditions } & $\begin{array}{c}\text { Film Thickness } \\
(\boldsymbol{\mu m})\end{array}$ & $\begin{array}{c}\text { Deposition Rate } \\
(\mathbf{n m} / \text { minute) }\end{array}$ & Roughness (nm) \\
\hline \multirow{2}{*}{$\begin{array}{c}\text { Deposition } \\
\text { Temperature }\left({ }^{\circ} \mathrm{C}\right)\end{array}$} & 100 & 1.00 & 33.33 & 1.35 \\
& 200 & 1.17 & 39.00 & 1.40 \\
& 300 & 1.30 & 43.33 & 3.00 \\
Sputtering Power & 400 & 1.78 & 59.33 & 3.10 \\
\hline$(\mathrm{W})$ & 90 & 0.62 & 20.67 & 3.46 \\
& 120 & 1.30 & 43.33 & 3.77 \\
\hline
\end{tabular}

Figure 2a presented the XRD pattern and slow scan rate spectra of the $\mathrm{Ge}_{0.07} \mathrm{GaN}$ films grown by $\mathrm{RF}$ sputtering at a different heating temperature in the range of $100-400{ }^{\circ} \mathrm{C}$ and at $120 \mathrm{~W}$ of $\mathrm{RF}$ power under the mixing of $\mathrm{Ar} / \mathrm{N}_{2}$ input gases. From the surveyed XRD, all $\mathrm{Ge}_{0.07} \mathrm{GaN}$ films deposited on $\mathrm{Si}$ (100) substrates at temperature $100-400{ }^{\circ} \mathrm{C}$ were polycrystalline including structural wurtzite, and Ge constituted the solid-state solution in the GaN crystal structure [16]. It could be clearly seen that these $\mathrm{Ge}_{0.07} \mathrm{GaN}$ films with a preferential $(10 \overline{10})$ growth plane had $(10 \overline{1} 0),(10 \overline{1} 1),(11 \overline{2} 0)$ and $(11 \overline{2} 2)$ peaks, and other secondary phases could not be found. At the higher heating temperature, the (1010) peak slightly shifted to the higher $2 \theta$ angle, and the (1010) peak of the deposited $\mathrm{Ge}_{0.07} \mathrm{GaN}$ films at 100, 200, 300 and $400^{\circ} \mathrm{C}$ was located at $32.25^{\circ}, 32.30^{\circ}, 32.36^{\circ}$ and $32.40^{\circ}$, respectively. Table 2 shows the parameters for the crystal structure of $\mathrm{Ge}_{0.07} \mathrm{GaN}$ films grown at different temperatures. The lattice constant $c$ slightly decreased from $5.21,5.20,5.18$ to $5.17 \AA$ and $a$ was $3.21,3.20,3.19$ and $3.18 \AA$, corresponding to GeGaN films made at the heating substrate temperatures of $100,200,300$ and $400{ }^{\circ} \mathrm{C}$, respectively. Additionally, cell volumes of Ge-0.07-GaN films sputtered at $100,200,300$, and $400^{\circ} \mathrm{C}$ were $46.57,46.13,45.70$ and 45.27 at $\AA^{3}$, respectively. From XRD data in Table 3, the dominant $(10 \overline{1} 0)$ peaks of the $100-400{ }^{\circ} \mathrm{C}$ deposited $\mathrm{Ge}_{0.07} \mathrm{GaN}$ films were slightly reduced with respect to the full width at half maxima (FWHM) values, i.e., $0.34^{\circ}, 0.30^{\circ}, 0.27^{\circ}$ and $0.25^{\circ}$ at $100,200,300$, and $400{ }^{\circ} \mathrm{C}$, respectively. Additionally, the 
crystalline size could be computed by the Scherer equation and the was significantly greater at higher heating temperature: $24.33,27.57,30.64$, and $33.09 \mathrm{~nm}$ for the $\mathrm{Ge}_{0.07} \mathrm{GaN}$ films deposited at 100, 200, 300 , and $400^{\circ} \mathrm{C}$, respectively. It could be believed that the heating temperature affected the structural crystallite of the film as the $\mathrm{Ge}_{0.07} \mathrm{GaN}$ films were deposited by RF sputtering at 100, 200, 300, and $400{ }^{\circ} \mathrm{C}$.

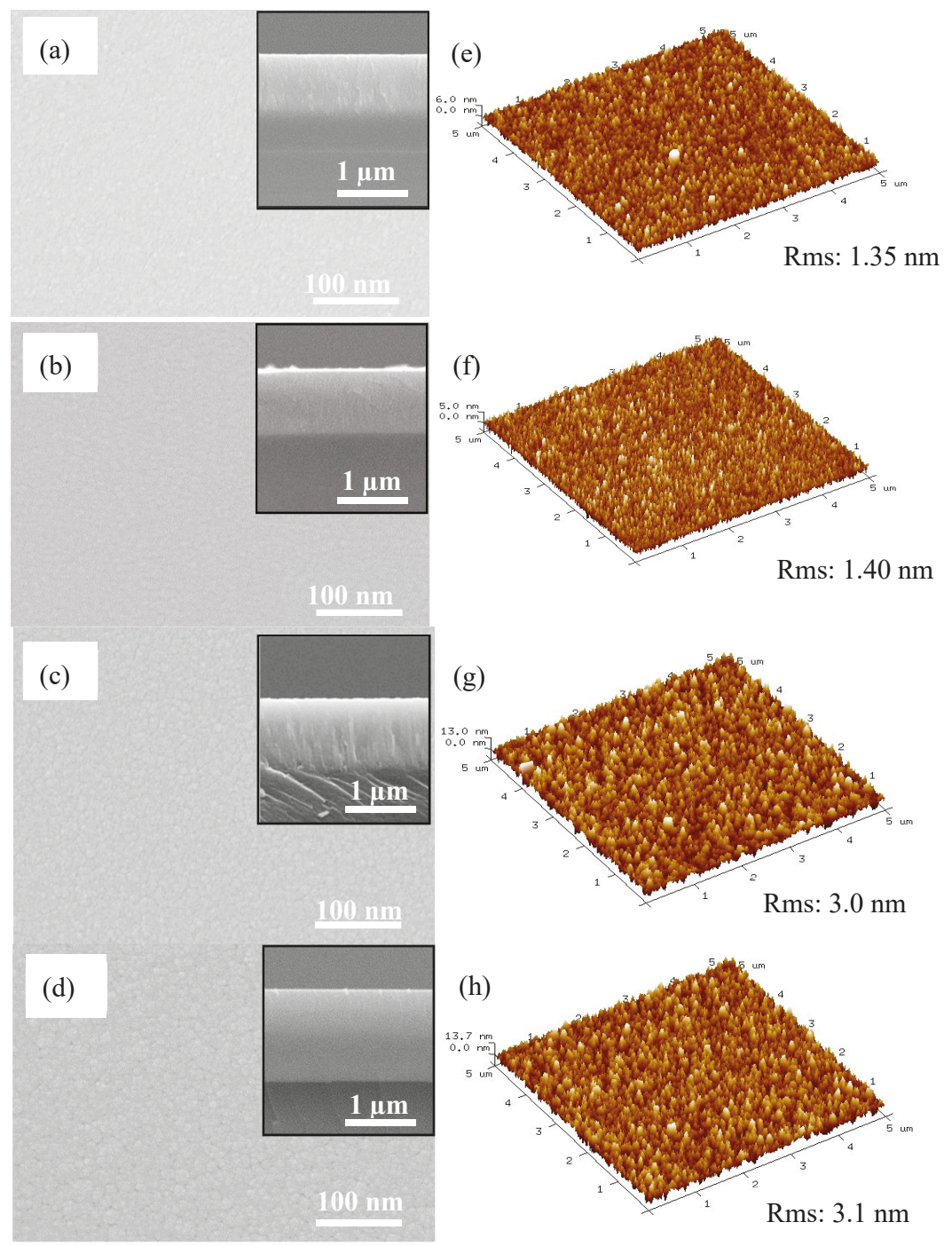

Figure 1. (a-d) SEM surface images and (e-h) $3 \mathrm{D}$ AFM morphologies of $\mathrm{Ge}_{0.07} \mathrm{GaN}$ films at (a,e) $100{ }^{\circ} \mathrm{C}$, $(\mathbf{b}, \mathbf{f}) 200^{\circ} \mathrm{C},(\mathbf{c}, \mathbf{g}) 30$ and $400{ }^{\circ} \mathrm{C}$. The insets are their individual cross-sectional images. 

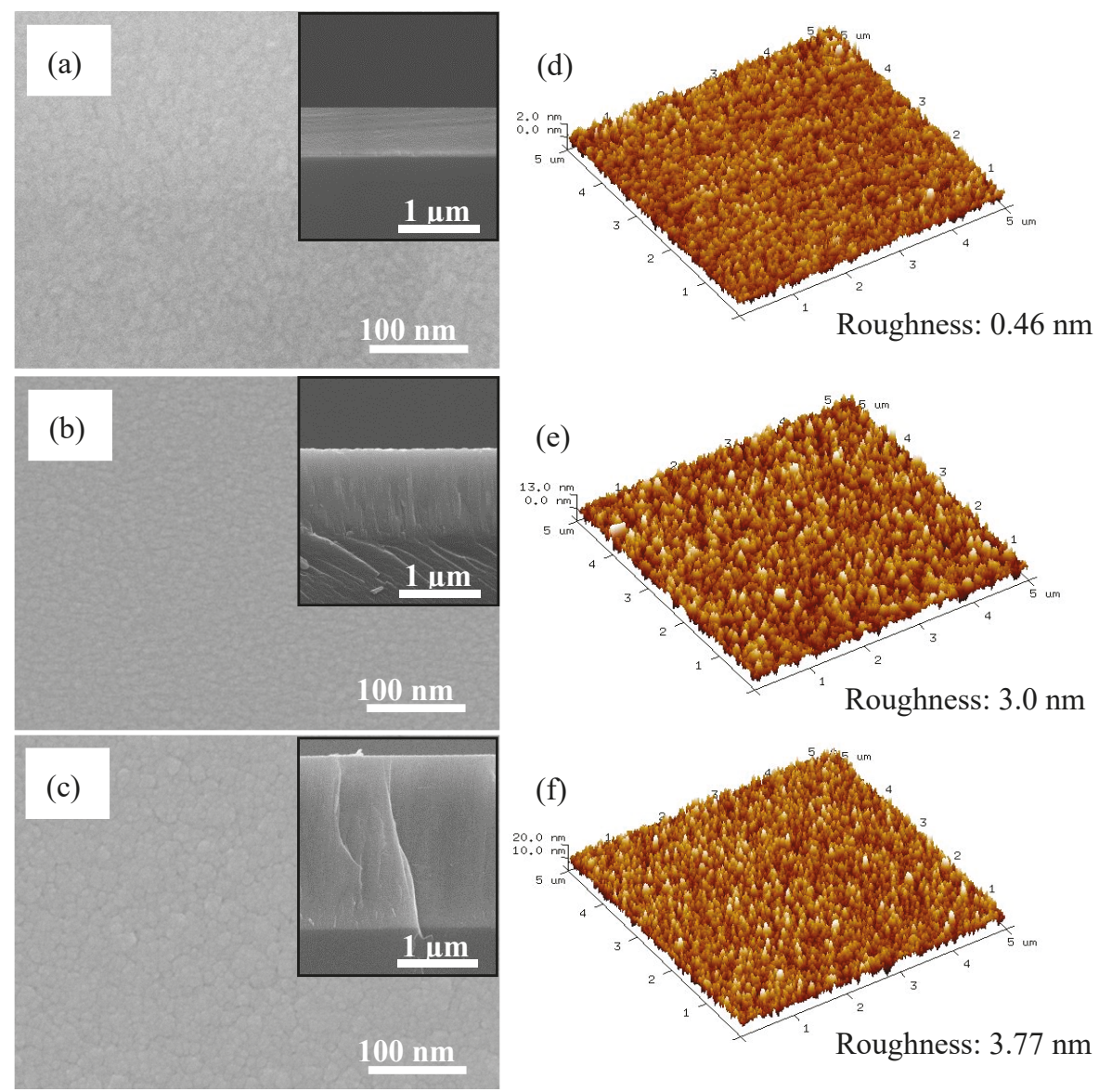

Figure 2. (a,b,d) SEM surface images and (e-g) 3D AFM morphologies of $\mathrm{Ge}_{0.07} \mathrm{GaN}$ films deposited at $(\mathbf{a}, \mathbf{d}) 90 \mathrm{~W},(\mathbf{b}, \mathbf{e}) 120 \mathrm{~W}$, and $(\mathbf{c}, \mathbf{f}) 150 \mathrm{~W}$ in $\mathrm{Ar} / \mathrm{N}_{2}$ atmosphere. The insets present their single cross-sectional images.

Table 3. Structure properties of $\mathrm{Ge}_{0.07} \mathrm{GaN}$ thin films at different sputtering powers and substrate temperature from $\mathrm{X}$-ray diffraction analyses.

\begin{tabular}{|c|c|c|c|c|c|c|c|}
\hline \multicolumn{2}{|c|}{ Sputtering Conditions } & \multirow{2}{*}{$\begin{array}{c}\left.\begin{array}{c}- \\
2 \theta(1010)\end{array}\right) \text { peak } \\
32.25\end{array}$} & \multirow{2}{*}{$\begin{array}{l}a(\AA) \\
3.21\end{array}$} & \multirow{2}{*}{$\begin{array}{c}c(\AA) \\
5.21\end{array}$} & \multirow{2}{*}{$\begin{array}{c}\begin{array}{c}\text { Volume } \\
\left(\AA^{3}\right)\end{array} \\
46.57\end{array}$} & \multirow{2}{*}{$\begin{array}{c}\begin{array}{c}\text { FWHM (1010) } \\
\text { (degree) }\end{array} \\
0.34\end{array}$} & \multirow{2}{*}{$\begin{array}{c}\begin{array}{c}\text { Crystallite } \\
\text { Size (nm) }\end{array} \\
24.33\end{array}$} \\
\hline & 100 & & & & & & \\
\hline \multirow{3}{*}{$\begin{array}{l}\text { Deposition } \\
\text { Temperature } \\
\quad\left({ }^{\circ} \mathrm{C}\right)\end{array}$} & 200 & 32.30 & 3.20 & 5.20 & 46.13 & 0.30 & 27.57 \\
\hline & 300 & 32.36 & 3.19 & 5.18 & 45.70 & 0.27 & 30.64 \\
\hline & 400 & 32.40 & 3.18 & 5.17 & 45.27 & 0.25 & 33.09 \\
\hline $\mathrm{RF}$ & 90 & 32.30 & 3.59 & 5.83 & 64.87 & 0.41 & 21.44 \\
\hline Sputtering & 120 & 32.36 & 3.38 & 5.49 & 54.26 & 0.27 & 33.04 \\
\hline Power (W) & 150 & 32.40 & 3.25 & 5.29 & 48.43 & 0.26 & 34.67 \\
\hline
\end{tabular}

The electrical properties of $\mathrm{Ge}_{0.07} \mathrm{GaN}$ films deposited at different temperatures in the range of $100-400^{\circ} \mathrm{C}$ and the $120 \mathrm{~W}$ of RF power were investigated by the Hall measurement system. In previous experiments, we reported that the $300^{\circ} \mathrm{C}$-sputtered $\mathrm{Ge}_{0.07} \mathrm{GaN}$ film achieved an electron concentration of $5.02 \times 10^{17} \mathrm{~cm}^{-3}$, mobility of $10.5 \mathrm{~cm}^{2} \cdot \mathrm{V}^{-1} \cdot \mathrm{s}^{-1}$, and carrier conductivity of $10.84 \mathrm{~S} \cdot \mathrm{cm}^{-1}$, and worked 
as an n-semiconductor layer [16]. From data displayed in Table 4 and Figure 3a, all sputtered $\mathrm{Ge}_{0.07} \mathrm{GaN}$ films at different growth temperatures from 100 to $400{ }^{\circ} \mathrm{C}$ remained $n$-type semiconductors. It could be explained that the compositional EDS data shown in Table 1 were responsible for the electrical properties of the $\mathrm{Ge}_{0.07} \mathrm{GaN}$ films. The $\mathrm{Ge}_{0.07} \mathrm{GaN}$ film at $100,200,300$, and $400{ }^{\circ} \mathrm{C}$ had an increase in electron concentration $\left(\mathrm{n}_{\mathrm{e}}\right)$ from $1.64 \times 10^{16}, 2.14 \times 10^{17}, 5.02 \times 10^{17}$ to $1.30 \times 10^{18} \mathrm{~cm}^{-3}$, and a decrease in mobility $(\mu)$ between $33,17,11$ and $7 \mathrm{~cm}^{2} \cdot \mathrm{V}^{-1} \cdot \mathrm{s}^{-1}$, respectively. It is believed that electron concentration could be a function of electrical conductivity and the as-deposited GeGaN at 100, 200, 300 and $400{ }^{\circ} \mathrm{C}$ maintained the increase in electronic conductivity corresponding to $0.09,0.58,0.88$ and $1.46 \mathrm{~S} \cdot \mathrm{cm}^{-1}$, respectively. The practical electrical properties of these $\mathrm{Ge}_{0.07} \mathrm{GaN}$ films illustrated that there were effects of heating substrate temperatures on film properties.

Table 4. Electrical properties of $\mathrm{Ge}_{0.07} \mathrm{GaN}$ films deposited at different temperatures.

\begin{tabular}{lcccccc}
\hline \multicolumn{2}{c}{ Sputtering Conditions. } & Type & $\begin{array}{c}\text { Concentration } \\
\mathbf{N}_{\mathbf{e}} \mathbf{~ c m}^{-\mathbf{3}}\end{array}$ & $\begin{array}{c}\text { Mobility } \boldsymbol{\mu} \\
\mathbf{c m}^{\mathbf{2}} \cdot \mathbf{V}^{-\mathbf{1}} \cdot \mathbf{s}^{-\mathbf{1}}\end{array}$ & $\begin{array}{c}\text { Conductivity } \boldsymbol{\sigma} \\
\mathbf{S} \cdot \mathbf{c m}^{-\mathbf{1}}\end{array}$ & $\begin{array}{c}\text { Bandgap } \\
\mathbf{e V}\end{array}$ \\
\hline Deposition & 100 & $\mathrm{n}$ & $1.64 \times 10^{16}$ & 33 & 0.09 & 3.14 \\
$\begin{array}{c}\text { Temperature } \\
\left({ }^{\circ} \mathrm{C}\right)\end{array}$ & 200 & $\mathrm{n}$ & $2.14 \times 10^{17}$ & 17 & 0.58 & 3.09 \\
& 300 & $\mathrm{n}$ & $5.02 \times 10^{17}$ & 11 & 0.88 & 3.05 \\
\hline \multirow{2}{*}{$\begin{array}{l}\text { Sputtering } \\
\text { Power }(\mathrm{W})\end{array}$} & 100 & $\mathrm{n}$ & $1.30 \times 10^{18}$ & 7 & 1.46 & 3.02 \\
\hline
\end{tabular}
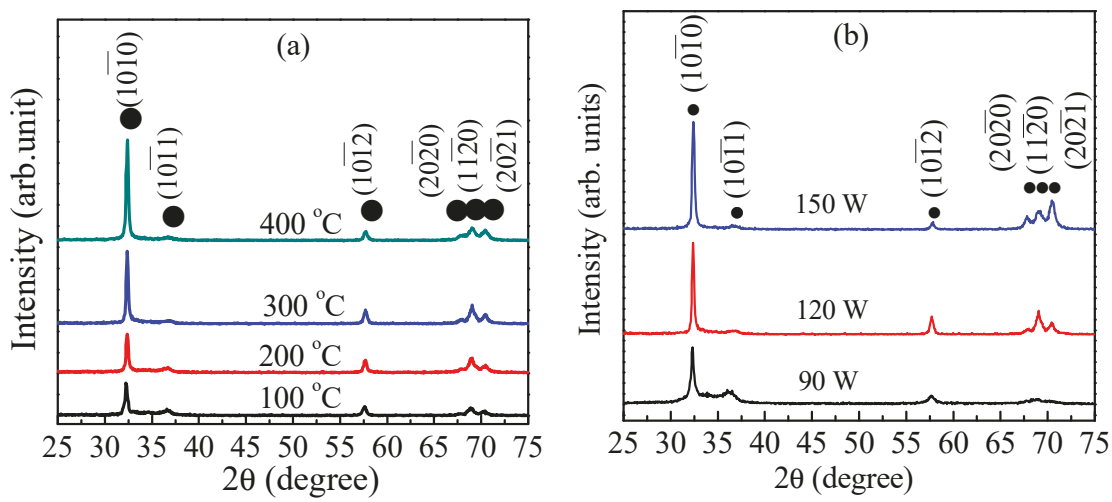

Figure 3. XRD patterns of $\mathrm{Ge}_{0.07} \mathrm{GaN}$ films deposited at (a) different growth temperatures; (b) different RF power $(90-150 \mathrm{~W})$ in an $\mathrm{Ar} / \mathrm{N}_{2}$ atmosphere.

The absorption of GeGaN films was studied by UV-Vis measurement at room temperature. The Equation (1) named the Tauc equation has been used to show the optical absorption coefficient and energy bandgap $\left(E_{\mathrm{g}}\right)$ of $\mathrm{Ge}_{0.07} \mathrm{GaN}$ films from the UV-Vis database.

$$
(\alpha h v)^{2}=A\left(h v-E_{\mathrm{g}}\right)
$$

where $A$ is a invariable number, $\alpha$ is the coefficient of optical absorption. From equation, the incident photon and the $\mathrm{Ge}_{0.07} \mathrm{GaN}$ films bandgap of energies were determined and listed for $h v$ and $E_{\mathrm{g}}$. Figure $4 \mathrm{a}$ and Table 4 show the plots of the $(\alpha h v)^{2}-h v$ curves and the bandgap values of $\mathrm{Ge}_{0.07} \mathrm{GaN}$ films deposited at different temperatures, which could be directly obtained by extrapolating the linear part of these curves. The $E_{\mathrm{g}}$ values from the extrapolated curves were 3.14, 3.09, 3.05, and $3.02 \mathrm{eV}$ for $\mathrm{Ge}_{0.07} \mathrm{GaN}$ films deposited at different temperatures from 100 to $400{ }^{\circ} \mathrm{C}$. 

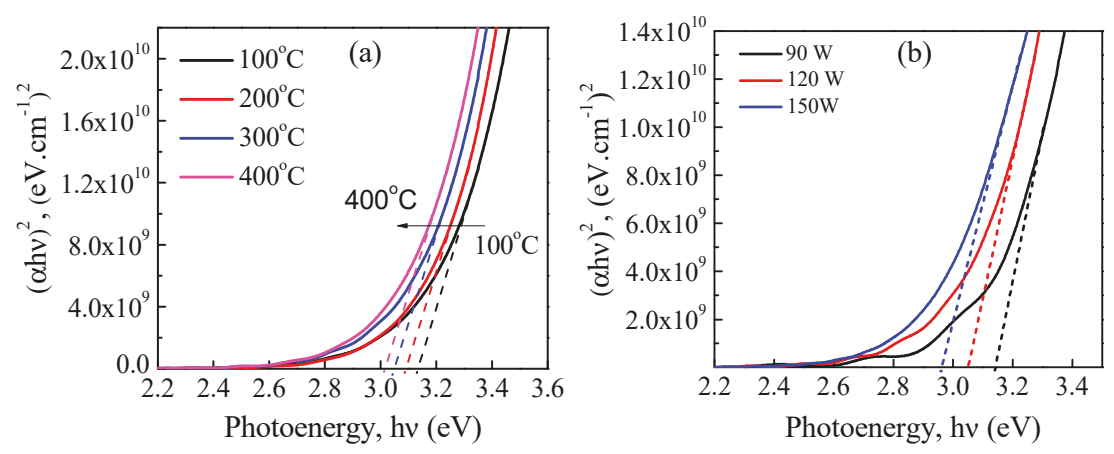

Figure 4. Plots of $(\alpha h v)^{2}$ vs. photon energy ( $\left.h v\right)$ for the optical band gap determination of the $\mathrm{Ge}_{0.07} \mathrm{GaN}$ films sputtered in a (a) deposition temperature $\left(100-400^{\circ} \mathrm{C}\right)$ range; (b) RF reactive sputtering range of 90-150 W.

3.2. Influences of RF Sputtering Power on the Electrical, Optical and Structural Properties of Ge-Doped Gan Thin Films

The composition of $\mathrm{Ge}_{0.07} \mathrm{GaN}$ films as-deposited at 90, 120 and $150 \mathrm{~W}$ RF sputtering power is shown in Table 1. The ratios of molar $[\mathrm{Ge}] /([\mathrm{Ge}]+[\mathrm{Ga}])$ were $7.3,8.5$ and 9.9 at \% for $\mathrm{Ge}_{0.07} \mathrm{GaN}$ films grown at 90, 120 and $150 \mathrm{~W}$, respectively. Under output RF power conditions, the Ge content in sputtered films increased with the RF power. Moreover, the nitrogen contents in these films were less than 50 at \%, which indicates that there was a nitrogen-deficiency state in $\mathrm{Ge}_{0.07} \mathrm{GaN}$ films at different sputtering powers.

The surface morphology and cross-section images of Ge-GaN films grown at different output RF sputtering powers are presented in Figure 4. The results of SEM images showed the smoothness surface and grains in nanometer size without voids and mechanical fracture phenomena. It is found that the higher sputtering power of deposition processes is the reason for the crystal grains having sufficient energy, causing the increase in the size of grains. From the cross-sectional patterns of $\mathrm{Ge}_{0.07} \mathrm{GaN}$ films at 90-150 W of RF power in Figure 5, film thickness increased from 0.62 to $2.5 \mu \mathrm{m}$ and explained the excellent adhesion, and no cracks or holes appeared at the surface between Si substrate and films. It can be observed that the thickness of the film increased as the film was deposited under a higher sputtering power. This means that the sputtering rate increased because the number of atoms deposited on the substrate increase and the film thickness will become thicker. From data in Table 3, the sputtering growth rate was 20.6743 .33 and $83.33 \mathrm{~nm} / \mathrm{min}$ corresponding to 90,120 and $150 \mathrm{~W}$ of deposition power. This experiment successful prepared $\mathrm{Ge}_{0.07} \mathrm{GaN}$ films under different sputtering powers without a buffer layer film. It can be seen from Figure 2 that GeGaN films have increased grain size as the RF sputtering power increased from 90 to $150 \mathrm{~W}$, which is due to the higher the sputtering power, the higher the current density of the plasma, and the free energy of the gas molecules, which increases, so that the opportunity to effectively hit the target increased, while the sputtered atoms have a large kinetic energy, arrived at the substrate with a high surface energy for grain growth, and increased the grain size. Using the Scherer equation, the crystalline size could be 24.33, 27.57, 30.64, and $33.09 \mathrm{~nm}$ for the $\mathrm{Ge}_{0.07} \mathrm{GaN}$ films grown at 90,120, and $150 \mathrm{~W}$, respectively. 

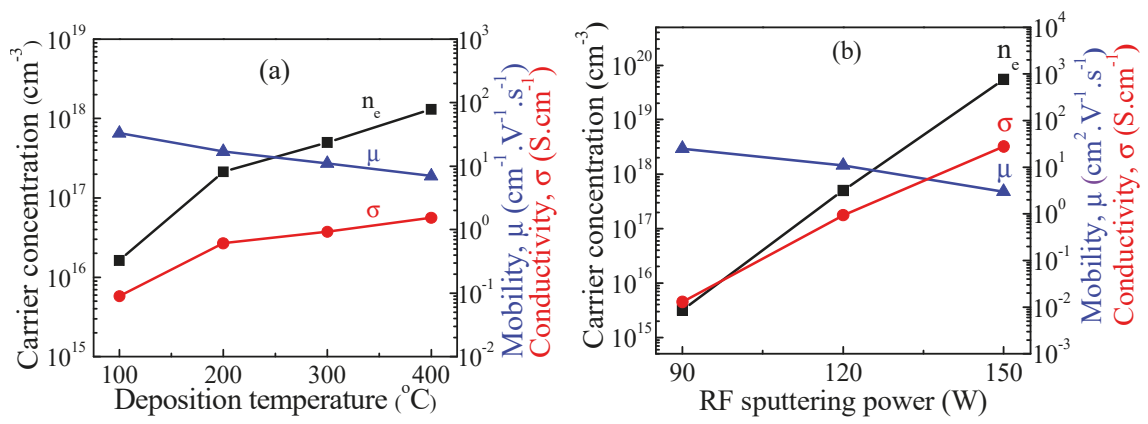

Figure 5. Electrical properties of $\mathrm{Ge}_{0.07} \mathrm{GaN}$ films deposited under a (a) heating substrate temperature range of $100-400{ }^{\circ} \mathrm{C}$, (b) $\mathrm{RF}$ sputtering power from 90 to $150 \mathrm{~W}$.

Under deposition power conditions of 90, 120 and $150 \mathrm{~W}$, the roughness of Ge-doped GaN films was $0.46,3.0$ and $3.2 \mathrm{~nm}$, respectively. It is explained that there is a relationship between the increase in sputtering power and the surface roughness of the film. The result of the roughness of films showed that higher bombardment of atoms from the target resulted in an increase in the deposition rate at higher sputtering power. Under higher output power, atoms have high surface movement energy to cause coarsening of grains and increases in the surface roughness of films.

Figure $3 \mathrm{~b}$ shows the $\mathrm{XRD}$ images of $\mathrm{Ge}_{0.07} \mathrm{GaN}$ thin films deposited under different output powers of 90,120 and $150 \mathrm{~W}$. The XRD results show that these $\mathrm{Ge}_{0.07} \mathrm{GaN}$ films exhibited a wurtzite structure. At the higher RF power of 120 and $150 \mathrm{~W}$, the sputtered $\mathrm{Ge}_{0.07} \mathrm{GaN}$ films were polycrystalline. However, there was deficient momentum between atoms and the substrate during the depositing process at $90 \mathrm{~W}$ $\mathrm{RF}$ power with respect to the low-quality crystallite of the $\mathrm{Ge}_{0.07} \mathrm{GaN}$ film. The $(10 \overline{1} 0),(10 \overline{1} 1),(10 \overline{1} 2)$, $(11 \overline{2} 0)$ and $(11 \overline{2} 2)$ diffraction peaks were found in Ge-GaN films, and no other secondary phase was detected. The peak positions of the $(10 \overline{1} 0)$ lattice plane were located at $32.30^{\circ}, 32.36^{\circ}$ and $32.40^{\circ}$ as the sputtering power was kept at 90,120 , and $150 \mathrm{~W}$, respectively. The $2 \theta$ angle of diffraction peaks slightly shifted higher at higher power. Table 2 shows all the calculated data from the XRD investigation. The a, c lattice constants and a unit cell volume of Ge-GaN films slightly degraded at higher RF power. While the c lattice constant slightly dropped from $5.83 \AA, 5.49 \AA$, and to $5.29 \AA$, there was a reduction in the a lattice constant from $3.59 \AA, 3.38 \AA$, and to $3.25 \AA$, with the cell volume of $64.87 \AA^{3}, 54.26 \AA^{3}$ to 48.43 $\AA^{3}$ corresponding to 90,120 and $150 \mathrm{~W}$, respectively. The full-width-half-maximum (FWHM) values of the $(10 \overline{1} 0)$ diffraction peaks of the $2 \theta$ value decreased from $0.41^{\circ}$ for $90 \mathrm{~W}$ power to $0.26^{\circ}$ for $150 \mathrm{~W}$ power. From XRD investigation at the higher sputtering power, the $\mathrm{Ge}_{0.07} \mathrm{GaN}$ films achieved a higher crystallinity quality. The XRD of $\mathrm{Ge}_{0.07} \mathrm{GaN}$ film deposited at $90 \mathrm{~W}$ showed the worst crystallinity because the low sputtering power condition created less Ge in the film. All the evidence indicates the formation of GeGaN films was affected by different sputtering powers.

Electrical properties of $\mathrm{Ge}_{0.07} \mathrm{GaN}$ films sputtered under output powers of 90,120 , and $150 \mathrm{~W}$ were investigated by the Hall effect measurement system at room temperature. The electron concentration $\left(n_{\mathrm{e}}\right)$, mobility $(\mu)$, and conductivity $(\sigma)$ are plotted in Figure $4 \mathrm{~b}$ and shown in Table 4 . All the $\mathrm{Ge}_{0.07} \mathrm{GaN}$ films deposited under different output powers presented as a semiconductor of the n-type. The electrical concentration $\left(n_{\mathrm{e}}\right)$ was $3.22 \times 10^{15}, 5.02 \times 10^{17}$ and $5.50 \times 10^{19} \mathrm{~cm}^{-3}$ while the electron mobility $(\mu)$ was 25,11 and $4 \mathrm{~cm}^{2} \cdot \mathrm{V}^{-1} \cdot \mathrm{s}^{-1}$ at the sputtering power of 90,120 , and $150 \mathrm{~W}$, respectively. The results from the experiments showed that carrier concentration increased with sputtering power. It can be explained that the sputtering power provides energy to the Ge solid solution in the GaN lattice. At low sputtering power, insufficient Ge solid solution precipitated at the grain boundaries prevents internal carrier transfer in films, which causes a lower free carrier concentration while Ge solid solution can be increased with the power upgrade. Additionally, the electrical conductivity $(\sigma)$ of the films was 
affected by carrier concentration $\left(n_{\mathrm{e}}\right)$ and mobility $(\mu)$, and electrical conductivity $(\sigma)$ was $0.012,0.84$ and $35.2 \mathrm{~S} \cdot \mathrm{cm}^{-1}$. The data show that the electrical conductivity increases as the output power increases.

The absorption coefficient and optical bandgap $\left(E_{\mathrm{g}}\right)$ of $\mathrm{Ge}_{0.07} \mathrm{GaN}$ films deposited at room temperature on a transparent glass plate at $90-150 \mathrm{~W}$ were tested by UV-Vis spectrometry. Figure $5 \mathrm{~b}$ shows the extrapolated linear part of the $(\alpha h v)^{2}-h v$ curves from which the optical bandgap of $\mathrm{Ge}_{0.07} \mathrm{GaN}$ films could be directly achieved, and the energy bandgap $E_{\mathrm{g}}$ was $3.14,3.05,2.96 \mathrm{eV}$ for $\mathrm{Ge}_{0.07} \mathrm{GaN}$ films under power conditions of 90, 120 and $150 \mathrm{~W}$, respectively. As the sputtering power increased, the energy gap gradually became smaller and decreased by $0.18 \mathrm{eV}$ from 90 watts to 150 watts. It is concluded that the increase in the RF power supplied sufficient energy to dissolve the Ge atoms into the lattice of $\mathrm{GaN}$, resulting in a decrease in the energy gap. As a result of electrical properties, it can be found that the carrier concentration increased with the increase in the sputtering power, and the film deposited at 150 watts has the highest carrier concentration and teh minimum energy gap.

\section{Conclusions}

$\mathrm{Ge}_{0.07} \mathrm{GaN}$ films were deposited on $\mathrm{Si}(100)$ substrates by employing radio frequency reactive magnetron sputtering technology at different temperature and RF power conditions. The characteristics and microstructure of these GeGaN films were studied thoroughly by AFM, SEM, XRD, UV-Vis spectrometry and the Hall effect measurement. The results showed that the $\mathrm{Ge}_{0.07} \mathrm{GaN}$ films remained in the polycrystalline structure and conductivity under the different growth conditions. The various sputtering conditions of the deposition process affected structural GeGaN films and resulted in heavy structural distortion. Compared with the sputtered film at different RF power values in the range of 90-150 W, the sputtered-150 W Ge $0.07 \mathrm{GaN}$ films achieved the lowest energy bandgap of $2.96 \mathrm{eV}$, the highest carrier concentration of $5.50 \times 10^{19} \mathrm{~cm}^{-3}$ and electrical conductivity of $35.2 \mathrm{~S} \cdot \mathrm{cm}^{-1}$, and $4 \mathrm{~cm}^{2} \cdot \mathrm{V}^{-1} \cdot \mathrm{s}^{-1}$ mobility. Besides, the analysis of $\mathrm{Ge}_{0.07} \mathrm{GaN}$ films at different substrate temperatures proved the influences of deposition temperature on the structure and properties of the films. From all investigated data, it could be believed that growth conditions of the RF reactive sputtering process affected the structure and properties of $\mathrm{Ge}_{0.07} \mathrm{GaN}$ films.

Author Contributions: Data curation, C.P.T. and T.T.A.T.; Methodology; Writing-original draft, investigation, C.P.T. and T.T.A.T., Formal analysis, Funding acquisition, Writing-review \& editing, C.P.T., T.T.A.T., N.H.V., K.A.T., T.T.V.S.N., K.V.N., and N.V.S.; Supervision, D.-H.K.

Funding: This research was funded by the Ministry of Science and Technology of the Republic of China under grant number 107-2221-E-011-141-MY3.

Conflicts of Interest: The authors declare no conflict of interest.

\section{References}

1. Akasaki, I.; Amano, H. Crystal growth and conductivity control of group III nitride semiconductors and their application to short wavelength light emitters. Jpn. J. Appl. Phys. 1997, 36, 5393-5408. [CrossRef]

2. Fujii, T.; Gao, Y.; Sharma, R.; Hu, E.L.; DenBaars, S.P.; Nakamura, S. Increase in the extraction efficiency of GaN-based light-emitting diodes via surface roughening. Appl. Phys. Lett. 2004, 84, 855-857. [CrossRef]

3. Pearton, S.J.; Ren, F.; Zhang, A.P.; Lee, K.P. Fabrication and performance of GaN electronic devices. Mater. Sci. Eng. R Rep. 2000, 30, 205-212. [CrossRef]

4. Tuan, T.T.A.; Kuo, D.-H. Characteristics of RF reactive sputter-deposited $\mathrm{Pt} / \mathrm{SiO}_{2} / \mathrm{n}-\mathrm{InGaN}$ MoS Schottky diodes. Mater. Sci. Semicond. Process. 2015, 30, 314-320. [CrossRef]

5. Tuan, T.T.A.; Kuo, D.-H.; Saragih, A.D.; Li, G.-Z. Electrical properties of RF-sputtered Zn-doped GaN films and $\mathrm{p}-\mathrm{Zn}-\mathrm{GaN} / \mathrm{n}-\mathrm{Si}$ hetero junction diode with low leakage current of $10^{-9} \mathrm{~A}$ and a high rectification ratio above $10^{5}$. Mater. Sci. Eng. B. 2017, 222, 18-25. [CrossRef]

6. Kuo, D.-H.; Liu, Y.-T. Characterization of quaternary $\mathrm{Zn} / \mathrm{Sn}$-codoped GaN films obtained with $\mathrm{Zn}_{x} \mathrm{Sn}_{0.04} \mathrm{GaN}$ targets at different $\mathrm{Zn}$ contents by the RF reactive magnetron sputtering technology. J. Mater. Sci. 2018, 53, 9099-9106. [CrossRef] 
7. Nakamura, S.; Mukai, T.; Senoh, M. Si- and Ge-doped GaN films grown with GaN buffer layers. Jpn. J. Appl. Phys. 1992, 31, 2883-2888. [CrossRef]

8. Hageman, P.R.; Schaff, W.J.; Janinski, J.; Liliental-Weber, Z. n-type doping of wurtzite GaN with germanium grown with plasma-assisted molecular beam epitaxy. J. Cryst. Growth. 2004, 267, 123-128. [CrossRef]

9. Colussi, M.L.; Baierle, R.J.; Miwa, R.H. Doping effects of C, Si and Ge in wurtzite [0001] GaN, AlN, and InN nanowires. J. Appl. Phys. 2011, 110, 033709. [CrossRef]

10. Oshima, Y.; Yoshida, T.; Watanabe, K.; Mishima, T. Properties of Ge-doped, high-quality bulk GaN crystals fabricated by hydride vapor phase epitaxy. J. Cryst. Growth. 2010, 312, 3569-3573. [CrossRef]

11. Kirste, R.; Hoffmann, M.P.; Sachet, E.; Bobea, M.; Bryan, Z.; Bryan, I.; Nenstiel, C.; Hoffmann, A.; Maria, J.-P.; Collazo, R.; et al. Ge doped GaN with controllable high carrier concentration for plasmonic applications. Appl. Phys. Lett. 2013, 103, 242107. [CrossRef]

12. Shikanaia, A.; Fukahori, H.; Kawakami, Y.; Hazu, K.; Sota, T.; Mitani, T.; Mukai, T.; Fujita, S. Optical properties of Si-, Ge- and Sn-doped GaN. Phys. Status. Solidi. B 2003, 235, 26-30. [CrossRef]

13. Dadgar, A.; Bläsing, J.; Diez, A.; Krost, A. Crack-free, highly conducting GaN layers on Si substrates by Ge doping. Appl. Phys. Express. 2011, 4, 011001. [CrossRef]

14. Fritze, S.; Dadgar, A.; Witte, H.; Bügler, M.; Rohrbeck, A.; Bläsing, J.; Hoffmann, A.; Krost, A. High Si and Ge n-type doping of GaN doping-Limits and impact on stress. Appl. Phys. Lett. 2012, 100, 122104. [CrossRef]

15. Özen, S.; Korkmaz, Ş.; Şenay, V.; Pat, S. The substrate effect on Ge doped GaN thin films coated by thermionic vacuum arc. J. Mater. Sci.: Mater. Electron. 2017, 28, 1288-1293. [CrossRef]

16. Thao, C.P.; Kuo, D.H. Electrical and structural characteristics of Ge-doped GaN thin films and its hetero-junction diode made all by RF reactive sputtering. Mater. Sci. Semicond Process 2018, 74, 336-341. [CrossRef]

17. Li, C.-C.; Kuo, D.-H. Effects of growth temperature on electrical and structural properties of sputtered GaN films with a cermet target. J. Mater. Sci.: Mater. Electron. 2014, 25, 1404-1409. [CrossRef]

18. Thao, C.P.; Kuo, D.-H.; Jan, D.-J. Codoping effects of the $\mathrm{Zn}$ acceptor on the structural characteristics and electrical properties of the Ge donor-doped $\mathrm{GaN}$ thin films and its hetero-junction diodes all made by reactive sputtering. Mater. Sci. Semicond. Process. 2018, 82, 126-134. [CrossRef]

19. Li, C.-C.; Kuo, D.-H. Material and technology developments of the totally sputtering-made $\mathrm{p} / \mathrm{n}$ GaN diodes for cost-effective power electronics. J. Mater. Sci.: Mater. Electron. 2014, 25, 1942-1948. [CrossRef]

20. Tuan, T.T.A.; Kuo, D.-H.; Li, C.C.; Yen, W.-C. Schottky barrier characteristics of Pt contacts to all sputtering-made n-type GaN and MoS diodes. J. Mater. Sci.: Mater. Electron. 2014, 25, 3264-3270. [CrossRef]

21. Ting, C.-W.; Thao, C.P.; Kuo, D.-H. Electrical and structural characteristics of tin-doped GaN thin films and its hetero-junction diode made all by RF reactive sputtering. Mater. Sci. Semicond Process. 2017, 59, 50-55. [CrossRef]

22. Kim, H.W.; Kim, N.H. Preparation of GaN films on Zno buffer layers by rf magnetron sputtering. Appl. Surf. Sci. 2004, 236, 192-197. [CrossRef]

23. Chyr, I.; Lee, B.; Chao, L.C.; Steckl, A.J. Damage generation and removal in the $\mathrm{Ga}^{+}$focused ion beam micromachining of GaN for photonic applications. J. Vac. Sci. Technol. 1999, 17, 3063-3067. [CrossRef]

(C) 2019 by the authors. Licensee MDPI, Basel, Switzerland. This article is an open access article distributed under the terms and conditions of the Creative Commons Attribution (CC BY) license (http://creativecommons.org/licenses/by/4.0/). 
Article

\title{
Electrical and Structural Properties of All-Sputtered $\mathrm{Al} / \mathrm{SiO}_{2} / p$-GaN MOS Schottky Diode
}

\author{
Tran Anh Tuan Thi ${ }^{1, *}$, Dong-Hau Kuo ${ }^{2, *}$, Phuong Thao Cao ${ }^{3}$, Pham Quoc-Phong ${ }^{3}$, \\ Vinh Khanh Nghi $^{3}$ and Nguyen Phuong Lan Tran ${ }^{4}$ \\ 1 School of Basic Sciences, Tra Vinh University, Tra Vinh 87000, Vietnam \\ 2 Department of Materials Science and Engineering, National Taiwan University of Science and Technology, \\ Taipei 10607, Taiwan \\ 3 School of Engineering and Technology, Tra Vinh University, Tra Vinh 87000, Vietnam; \\ cpthao@tvu.edu.vn (P.T.C.); phongpham@tvu.edu.vn (P.Q.-P.); nghivinhkhanh@tvu.edu.vn (V.K.N.) \\ 4 School of Engineering and Technology, Can Tho University, Can Tho 94000, Vietnam; tnplan@ctu.edu.vn \\ * Correspondence: thitrananhtuan@tvu.edu.vn (T.A.T.T.); dhkuo@mail.ntust.edu.tw (D.-H.K.); \\ Tel.: +886-2-2730-3291 (D.-H.K.)
}

Received: 22 September 2019; Accepted: 14 October 2019; Published: 21 October 2019

\begin{abstract}
The all-sputtered $\mathrm{Al} / \mathrm{SiO}_{2} / p$-GaN metal-oxide-semiconductor (MOS) Schottky diode was fabricated by the cost-effective radio-frequency sputtering technique with a cermet target at $400{ }^{\circ} \mathrm{C}$. Using scanning electron microscope (SEM), the thicknesses of the electrodes, insulator $\mathrm{SiO}_{2}$ layer, and $p-\mathrm{GaN}$ were found to be $\sim 250 \mathrm{~nm}, 70 \mathrm{~nm}$, and $1 \mu \mathrm{m}$, respectively. By Hall measurement of a $p-\mathrm{Mg}-\mathrm{GaN}$ film on an $\mathrm{SiO}_{2} / \mathrm{Si}(100)$ substrate at room temperature, the hole's concentration $\left(N_{p}\right)$ and carrier mobility $(\mu)$ were found to be $N_{p}=4.32 \times 10^{16} \mathrm{~cm}^{-3}$ and $\mu=7.52 \mathrm{~cm}^{2} \cdot \mathrm{V}^{-1} \cdot \mathrm{s}^{-1}$, respectively. The atomic force microscope (AFM) results showed that the surface topography of the $p$-GaN film had smoother, smaller grains with a root-mean-square $(\mathrm{rms})$ roughness of $3.27 \mathrm{~nm}$. By $I-V$ measurements at room temperature (RT), the electrical properties of the diode had a leakage current of $\sim 4.49 \times 10^{-8}$ A at $-1 \mathrm{~V}$, a breakdown voltage of $-6 \mathrm{~V}$, a turn-on voltage of $\sim 2.1 \mathrm{~V}$, and a Schottky barrier height (SBH) of $0.67 \mathrm{eV}$. By $C-V$ measurement at RT, with a frequency range of $100-1000 \mathrm{KHz}$, the concentration of the diode's hole increased from $3.92 \times 10^{16} \mathrm{~cm}^{-3}$ at $100 \mathrm{kHz}$ to $5.36 \times 10^{16} \mathrm{~cm}^{-3}$ at $1 \mathrm{MHz}$, while the Fermi level decreased slightly from 0.109 to $0.099 \mathrm{eV}$. The SBH of the diode at RT in the $C-V$ test was higher than in the $I-V$ test because of the induced charges by dielectric layer. In addition, the ideality factor $(n)$ and series resistance $\left(R_{s}\right)$ determined by Cheung's and Norde's methods, other parameters for MOS diodes were also calculated by $C-V$ measurement at different frequencies.
\end{abstract}

Keywords: MOS Schottky diode; $\mathrm{SBH}$; $I-V$ measurement; $C-V$ measurement; Cheung's and Norde's methods

\section{Introduction}

GaN-based semiconductor materials are currently of interest for the fabrication of electronic devices such as the metal-semiconductor (MS) and MOS Schottky diodes, light-emitting diodes (LEDs), photo-detector, metal-oxide-semiconductor field-effect transistors (MOSFETs), and heterojunction field-effect transistors (HFETs). [1-5]. Previous studies created the thin, high-quality insulator layer between the metal and semiconductor that is used to create a metal-oxide-semiconductor (MOS) structure, which was an important factor for the high-performance of MOS devices [6-10]. Researchers investigated the contact of MOS layers via various approaches, e.g., $\mathrm{Al} / \mathrm{HfO}_{2} / p$-Si [7], Pt/oxide/n-InGaP [10], $\mathrm{Pt} / \mathrm{SiO}_{2} / n$-InGaN [11], $\mathrm{Pd} / \mathrm{NiO} / \mathrm{GaN}$ [12], $\mathrm{Au} / \mathrm{SiO}_{2} / n$-GaN [13], $\mathrm{Au} / \mathrm{SnO}_{x} / n$-LTPS/glass [14], $\mathrm{Pt} / \mathrm{SiO}_{2} / n$-GaN [6,15], Pt/Oxide/Al ${ }_{0.3} \mathrm{Ga}_{0.7} \mathrm{As}$ [16], 
$\mathrm{Pd} / \mathrm{HfO}_{2} / \mathrm{GaN}$ [17], and $\mathrm{Al} / \mathrm{SnO}_{2} / p$-Si (111) [18]. Due to the presence of the oxide layer, several parameters can be applied to improve the characteristics of electronic devices. Bengi et al. reported the parameters of the $\mathrm{Al} / \mathrm{HfO}_{2} / p$-Si MOS device, which was tested by $\mathrm{C}-V$ measurement. Their SBHs were shown from 0.17 to $0.98 \mathrm{eV}$, in the temperature range 300-400 K [7]. Karadeniz et al. investigated the $\mathrm{Al} / \mathrm{SnO}_{2} / p-\mathrm{Si}$ (111) diode using spray deposition. The MOS diode showed a Schottky barrier height (SBH) of $0.52 \mathrm{~V}$, an ideality factor of 2.4, and series resistance of $66 \Omega$ [18]. Liu et al. studied the influence of hydrogen adsorption on the $\mathrm{Pd} / \mathrm{AlGaN}$-based $\mathrm{MOS}$ diode with $\mathrm{SiO}_{2}$ passivation [19]. Their SBHs were reduced from 0.98 to $0.75 \mathrm{eV}$ under exposure to a $1 \% \mathrm{H}_{2} / \mathrm{air}$ gas.

In this study, the radio-frequency (RF) reactive sputtering technique was used to design the $\mathrm{Al} / \mathrm{SiO}_{2} / p$-GaN MOS Schottky diode because of advantages such as low deposition temperature, low cost, and safety $[3,6,11]$. With the support of the parameters and using the RF technique, our diode was fabricated below $400{ }^{\circ} \mathrm{C}$. The characteristics of the MOS Schottky diode were tested using $I-V$ and $C-V$ measurements. The parameters of the diode were calculated by thermionic emission (TE) mode using Cheung's and Norde's methods.

\section{Materials and Methods}

Figure 1 shows the modeling of the $\mathrm{Al} / \mathrm{SiO}_{2} / p-\mathrm{GaN}$ MOS Schottky diode based on $p-\mathrm{GaN}$ film. First, for the Schottky contacts, an $\mathrm{Al}$ layer was sputtered on an $\mathrm{SiO}_{2} / \mathrm{Si}(100)$ substrate at $200{ }^{\circ} \mathrm{C}$ for 20 min using a pure $\mathrm{Al}(99.99 \%)$ target, and RF power of $80 \mathrm{~W}$. To construct the MOS Schottky diodes, an interlayer between $\mathrm{Al}$ and $p-\mathrm{GaN}$ was designed by depositing $\mathrm{SiO}_{2}$. The $\mathrm{SiO}_{2}$ film was sputtered on an $\mathrm{Al} / \mathrm{SiO}_{2} / \mathrm{Si}(100)$ substrate at $100{ }^{\circ} \mathrm{C}$ for 10 min using a quartz target. The RF power remained at $80 \mathrm{~W}$ and the Ar atmosphere at a flow rate of $5 \mathrm{sccm}$. Second, the $\mathrm{Mg}-\mathrm{GaN}$ films were deposited onto $\mathrm{SiO}_{2} / \mathrm{Al} / \mathrm{SiO}_{2} / \mathrm{Si}(100)$ and $\mathrm{SiO}_{2} / \mathrm{Si} \mathrm{(100)} \mathrm{substrates} \mathrm{at} 400{ }^{\circ} \mathrm{C}$ for $40 \mathrm{~min}$. The $\mathrm{RF}$ power of was kept at $150 \mathrm{~W}$ with a gas mixture of $\mathrm{Ar}$ and $\mathrm{N}_{2}$ and a flow rate of $5 \mathrm{sccm}$ for each. The 2-inch Mg-GaN target had an $[\mathrm{Mg}] /([\mathrm{Ga}]+[\mathrm{Mg}])$ molar ratio of $10 \%$ and was made via hot pressing. Finally, a Pt-Omhic contact with a size of $1 \mathrm{~mm}^{2}$ was deposited, at $200{ }^{\circ} \mathrm{C}$ for $20 \mathrm{~min}$, with a pure $\mathrm{Pt}(99.99 \%)$ target using a stainless mask.

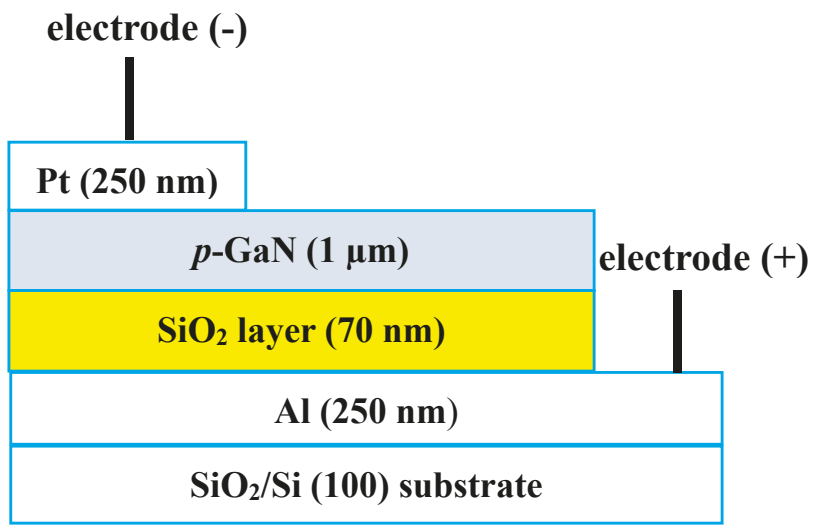

Figure 1. The modeling of the as-deposited $\mathrm{Al} / \mathrm{SiO}_{2} / p-\mathrm{GaN}$ MOS Schottky diode.

The composition analysis and surface topographies of the $p$-GaN films were determined via SEM and EDS (JSM-6500F, JEOL, Tokyo, Japan), AFM (Dimension Icon, Bruker, Tokyo, Japan). The hole's concentration $\left(N_{p}\right)$ and the mobilities $(\mu)$ of the $p$-GaN film were calculated by Hall measurement (HMS-2000, Ecopia, Tokyo, Japan). The $I-V$ and $C-V$ measurement of the MOS Schottky diode were tested using a semiconductor device analyzer (Agilent, B1500A, Santa Clara, CA, USA) at RT. All the parameters of the MOS Schottky diode were considered by thermionic emission (TE) mode using Cheung's and Norde's methods. 


\section{Results and Discussion}

\subsection{Structural and Surface Morphological Characteristics}

By Hall measurement of the $p-\mathrm{Mg}-\mathrm{GaN}$ film on the $\mathrm{SiO}_{2} / \mathrm{Si}(100)$ substrate at RT, the hole's concentration $\left(N_{p}\right)$ and carrier mobility $(\mu)$ were found to be $N_{p}=4.32 \times 10^{16} \mathrm{~cm}^{-3}$ and $\mu=7.52 \mathrm{~cm}^{2} \cdot \mathrm{V}^{-1} \cdot \mathrm{s}^{-1}$, respectively. Using SEM, the thicknesses of both the electrodes and the $\mathrm{SiO}_{2}$ layer were found to be 250 and $70 \mathrm{~nm}$, respectively.

Figure 2a shows the SEM surface morphologies of the $p-\mathrm{GaN}$ films sputtered on the $\mathrm{SiO}_{2} / \mathrm{Si}(100)$ substrate. With EDS analysis results, the ratio of $[\mathrm{Mg}] /([\mathrm{Ga}]+[\mathrm{Mg}])$ was $10.2 \%$ for the $p-\mathrm{GaN}$ film. This indicated that the $p$-Mg-GaN film deposited at $400{ }^{\circ} \mathrm{C}$ with up to $10 \% \mathrm{Mg}$ displayed continuous smoothness without cracks and pores. The inset shows a cross-sectional image, with a thickness of $1 \mu \mathrm{m}$ for the $p$-GaN film. Figure $2 \mathrm{~b}$ shows the surface topography of the as-deposited $\mathrm{Mg}-\mathrm{GaN}$ films on the $\mathrm{SiO}_{2} / \mathrm{Si}(100)$ substrate tested by AFM measurement. The surface topography showed smoother and smaller grains and the root-mean-square (rms) roughness of the films was found to be $3.27 \mathrm{~nm}$. The EDS compositions, SEM surface morphologies and XRD patterns of the $p$-Mg-GaN film obtained with cermet targets at different $\mathrm{Mg}$ contents can be found in our previous works [20,21]. The positive surface conditions of $\mathrm{Mg}-\mathrm{GaN}$ layer together with the insulator $\mathrm{SiO}_{2}$ layer were the important factors for determining the electrical properties of the MOS Schottky diodes.
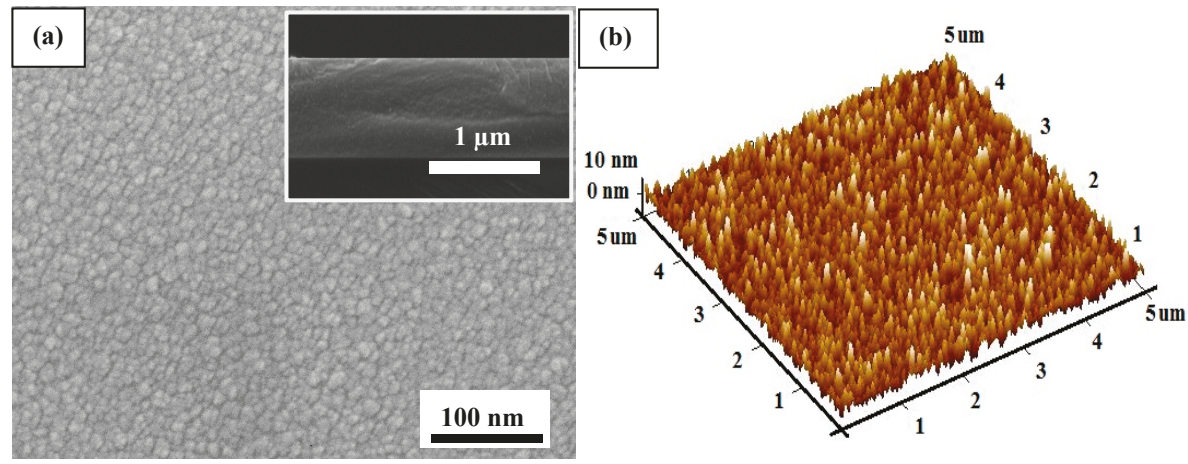

Figure 2. (a) SEM surface image and (b) three-dimensional AFM topographies of the $p$-GaN film deposited on the $\mathrm{SiO}_{2} / \mathrm{Si} \mathrm{(100)} \mathrm{substrate.} \mathrm{The} \mathrm{inset} \mathrm{is} \mathrm{the} \mathrm{cross-sectional} \mathrm{image} \mathrm{of} \mathrm{the} p$-GaN film.

\subsection{Current-Voltage (I-V) Characteristics}

Figure 3a displays the $I-V$ plot of the $\mathrm{Al} / \mathrm{SiO}_{2} / p-\mathrm{GaN}$ MOS Schottky diode measured at RT. The Figure $3 \mathrm{~b}$ shows the $\ln I-V$ semilogarithmic view of the diode. From the $I-V$ data, tested with a voltage range of $(-6 \mathrm{~V} ;+6 \mathrm{~V})$ and a leakage current of $-1 \mathrm{~V}$, the turn-on voltage of the diode was determined to be $\sim 4.49 \times 10^{-6} \mathrm{~A} / \mathrm{cm}^{2}$ and $2.3 \mathrm{~V}$. 


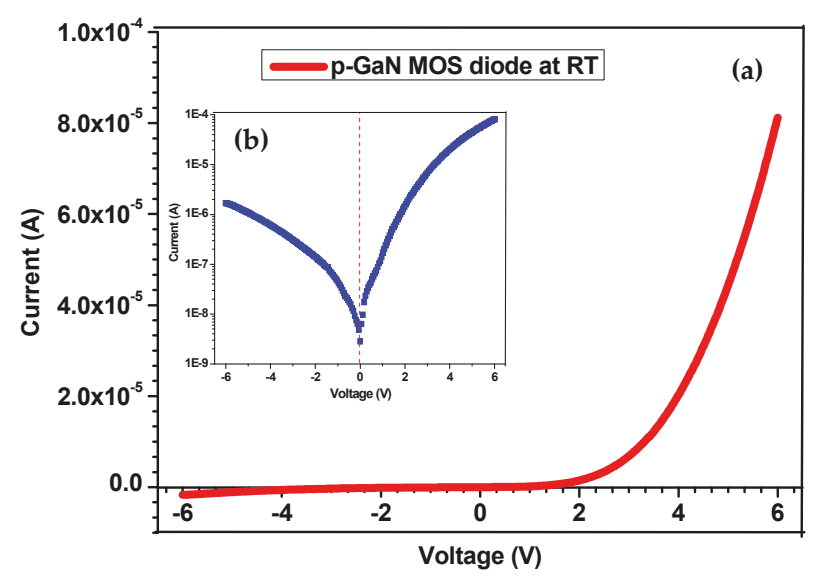

Figure 3. (a) $I-V$ plot of the as-deposited $\mathrm{Al} / \mathrm{SiO}_{2} / p-\mathrm{GaN}$ MOS Schottky diode tested at $\mathrm{RT}$, (b) the forward and reverse $\ln I-V$ characteristics of diodes.

According to the thermionic emission (TE) mode (for $q V>3 \mathrm{kT}$ ), the electrical properties of the Schottky diode can be described as [6,11,22]:

$$
I=I_{0} \exp \left[q\left(V-I R_{s}\right) / n \mathrm{k} T\right]
$$

The SBH can be expressed by $[1,5,21]$ :

$$
\phi_{\mathrm{B}}=\frac{\mathrm{k} T}{q} \ln \left(\frac{A \mathrm{~A}^{*} T^{2}}{I_{0}}\right)
$$

where $I_{0}$ is the saturation current, $V$ is the applied voltage, $R_{S}$ is the series resistance, $n$ is the ideality factor, $T$ is the measured temperature in Kelvin, $q$ is the electronic charge, $\mathrm{k}$ is the Boltzmann constant, $\phi_{\mathrm{B}}$ is the Schottky barrier height (SBH), $\mathrm{A}^{*}$ is the Richardson constant, $A$ is the contact area of the diode, and $A^{*}$ is the effective. The saturation current $I_{0}$ was defined by the intersection between the interpolated straight lines of the linear region and the current axis.

Using a stainless-steel mask with a square opening, the electrodes of our diode were measured at $1 \mathrm{~mm}^{2}$. The $\mathrm{A}^{*}$ value was $26.4 \mathrm{~A} \cdot \mathrm{cm}^{-2} \cdot \mathrm{K}^{-2}$ (based on effective mass $m^{*}=0.22 \times m_{e}$ for $\mathrm{GaN}, m_{e}$ is electron mass) $[4,5,13]$. The ideality factor $(n)$ from Equation (1) can be determined by $[5,21,23]$ :

$$
n=\frac{q}{\mathrm{k} T}\left(\frac{\mathrm{d} V}{\mathrm{~d}(\ln I)}\right)
$$

Based upon Equations (1) and (2), the SBH of the diode was $0.67 \mathrm{~V}$, while the ideality factor $n$, based on Equation (3), was 3.32. According to Cheung's method, the series resistance $R_{S}$ and ideality factor can be found by the intersecting slope from the linear region of the $\mathrm{d} V / \mathrm{d}(\ln I)$ vs. the $I$ plots [11,22,24-26]:

$$
\frac{\mathrm{d} V}{\mathrm{~d}(\ln I)}=\frac{n \mathrm{k} T}{q}+I R_{S}
$$

As shown in Figure 4, a calculation based on Equation (4) showed that the values of $R_{S}$ and $n$ were $5914 \Omega$ and 3.51, respectively. Our MOS Schottky diode had high series resistance because there was an insulator $\mathrm{SiO}_{2}$ layer of $70 \mathrm{~nm}$ between the metal and semiconductor. 


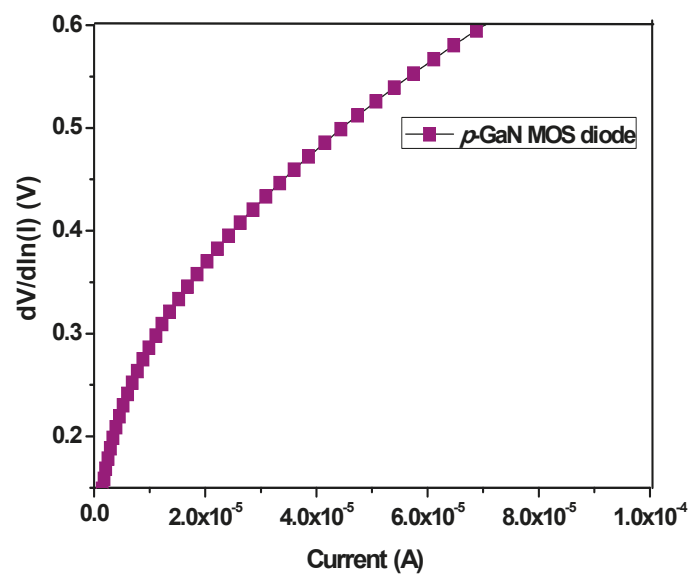

Figure 4. Plot of $\mathrm{d} V / \mathrm{d} \ln (I)$ versus $I$ for the as-deposited $\mathrm{Al} / \mathrm{SiO}_{2} / p-\mathrm{GaN}$ MOS Schottky diode.

The Norde method was also used to calculate the effective SBH of the diodes. The Norde function is described as the $\mathrm{F}(V, I)$ vs. the voltage $V$. It is given by $[6,27]$ :

$$
\mathrm{F}(V, I)=\frac{V}{\gamma}-\frac{\mathrm{k} T}{q} \ln \left(\frac{I}{A \mathrm{~A}^{*} T^{2}}\right)
$$

The effective $\mathrm{SBH} \phi_{\mathrm{B}}$ is obtained by:

$$
\phi_{\mathrm{B}}=\mathrm{F}\left(V_{\min }\right)+\frac{V_{\min }}{\gamma}-\frac{\mathrm{k} T}{q}
$$

where $\gamma$ is the first integer (dimensionless) is higher than $n, \mathrm{~F}\left(V_{\min }\right)$ is the min value of $\mathrm{F}(V)$, and $V_{\min }$ is the corresponding voltage $[27,28]$.

Figure 5 displays the plot of $\mathrm{F}(V)$ vs. the $V$ of the $\mathrm{Al} / \mathrm{SiO}_{2} / p-\mathrm{GaN}$ MOS Schottky diode measured at RT. Based on Equations (5) and (6), the SBH value was $0.78 \mathrm{eV}$ for the device. Table 1 lists all the parameters of the diode, calculated by $I-V$ test, and Cheung's and Norde's methods.

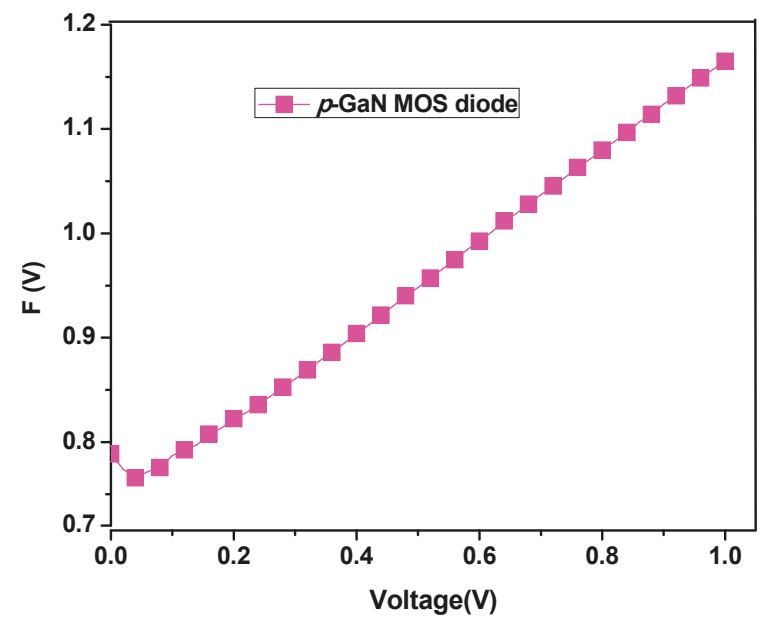

Figure 5. Characterization of the $\mathrm{F}(V, I)$ vs. $V$ for the as-deposited $\mathrm{Al} / \mathrm{SiO}_{2} / p-\mathrm{GaN}$ MOS Schottky diode. 
Table 1. The parameters of the $I-V$ characteristics of the $\mathrm{Al} / \mathrm{SiO}_{2} / p-\mathrm{MOS}$ Schottky diode at room temperature.

\begin{tabular}{|c|c|c|c|c|c|c|}
\hline \multirow[t]{2}{*}{ Sample } & \multirow{2}{*}{$\begin{array}{l}\text { Leakage Current } \\
\text { (A) at }-1(\mathrm{~V})\end{array}$} & \multicolumn{2}{|c|}{$\begin{array}{l}\text { Schottky Barrier } \\
\text { Height }(\mathrm{SBH})(\mathrm{eV})\end{array}$} & \multirow{2}{*}{$\begin{array}{c}\text { From } I-V \\
n\end{array}$} & \multicolumn{2}{|c|}{$\begin{array}{l}\text { Cheungs' Function } \\
\text { dV/dln }(I)-I\end{array}$} \\
\hline & & $I-V$ & Norde & & $R_{s}(\Omega)$ & $n$ \\
\hline As-dep. & $4.49 \times 10^{-8}$ & 0.67 & 0.78 & 3.32 & 5914 & 3.51 \\
\hline
\end{tabular}

\subsection{Capacitance-Voltage ( $\mathrm{C}-\mathrm{V})$ Characteristics}

The capacitance-voltage $(C-V)$ measurement of our diode was expressed and tested at room temperature, with a frequency range of $100 \mathrm{kHz}-1 \mathrm{MHz}$. The $C-V$ relationship of diodes can be expressed by $[4,9,13]$ :

$$
\begin{gathered}
\frac{1}{C^{2}}=\frac{2\left(V_{b i}-\frac{\mathrm{k} T}{q}-V\right)}{q \varepsilon_{\mathrm{S}} N_{p} A^{2}} \\
N_{p}=\frac{2}{q \varepsilon_{\mathrm{s}} A^{2}}\left[-\frac{1}{\mathrm{~d}\left(1 / \mathrm{C}^{-2}\right) / \mathrm{d} V}\right]
\end{gathered}
$$

where $N_{p}$ is hole concentration, $V$ is the flat band voltage, $A$ is the area of the diode, and $\varepsilon_{\mathrm{s}}$ is the permittivity of the semiconductor $\left(\varepsilon_{\mathrm{S}}=9.5 \times \varepsilon_{\mathrm{o}}\right.$ for $\mathrm{GaN}$, $\varepsilon_{\mathrm{o}}$ is electric constant) [4,13]. $V_{0}$ is determined by the plot of $1 / C^{2}$ vs. $V$. The potential $V_{b i}$ is calculated from $V_{0}$ by $[4,5,11]$ :

$$
V_{b i}=V_{0}+\frac{\mathrm{k} T}{q}
$$

The SBH $\phi_{\mathrm{CV}}$ from the $C-V$ measurement is given by $[13,14,18]$ :

$$
\phi_{\mathrm{CV}}=V_{b i}+E_{F}-\Delta \Phi_{b}
$$

where $E_{F}$ is the energy of Fermi level. This is given by $[9,11,13]$ :

$$
E_{F}=\frac{\mathrm{k} T}{q} \ln \left(\frac{N_{c}}{N_{p}}\right)
$$

Based on the $m^{*}=0.22 \times m_{e}$ for GaN, $N_{c}$ is the density of states in the conduction band edge. It is expressed by $[2,5,7]$ :

$$
N_{c}=2\left(\frac{2 \pi m^{*} k T}{h^{2}}\right)^{3 / 2}
$$

where $h$ is Plank constant. The $\Delta \Phi_{b}$ is the image force-induced barrier lowering. It is given by $[7,9,13]$ :

$$
\Delta \Phi_{b}=\left[\frac{q E_{m}}{4 \pi \varepsilon_{s} \varepsilon_{0}}\right]^{1 / 2}
$$

where $E_{m}$ is the maximum electric field and given by $[9,13]$ :

$$
E_{m}=\left[\frac{2 q N_{p} V_{0}}{\varepsilon_{\mathrm{s}} \varepsilon_{0}}\right]^{1 / 2}
$$

Figure 6a shows the plotted $C-V$ measurement of the MOS Schottky diode tested at the frequency range $100 \mathrm{KHz}-1 \mathrm{MHz}$. The Figure $6 \mathrm{~b}$ is the electrical properties of the diode, which was measured at a frequency of $1 \mathrm{MHz}$ with an alternating current (AC) modulation of $100 \mathrm{mV}$. Figure 7 shows the characterization of $1 / C^{2}$ vs. $V$ as a function of the $p$-MOS Schottky diode tested at different frequencies. 
The $x$-intercept of the $1 / C^{2}$ vs. $V$ plot determined $V_{0}$ from the straight lines for the downward region at the reverse bias $[4,5,7,11]$.

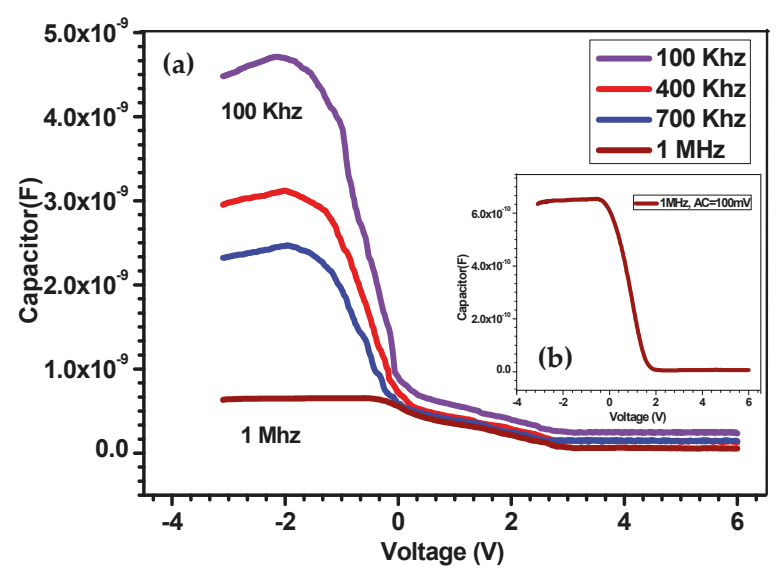

Figure 6. (a) Plot $\mathrm{C}-V$ measurement for the as-deposited $\mathrm{Al} / \mathrm{SiO}_{2} / p-\mathrm{GaN}$ MOS Schottky diode measurement at different frequencies between $100 \mathrm{kHz}$ and $1 \mathrm{MHz}, \mathrm{AC}=100 \mathrm{mV}$. (b) The electrical properties of the diode was measured at frequency of $1 \mathrm{MHz}, \mathrm{AC}=100 \mathrm{mV}$.

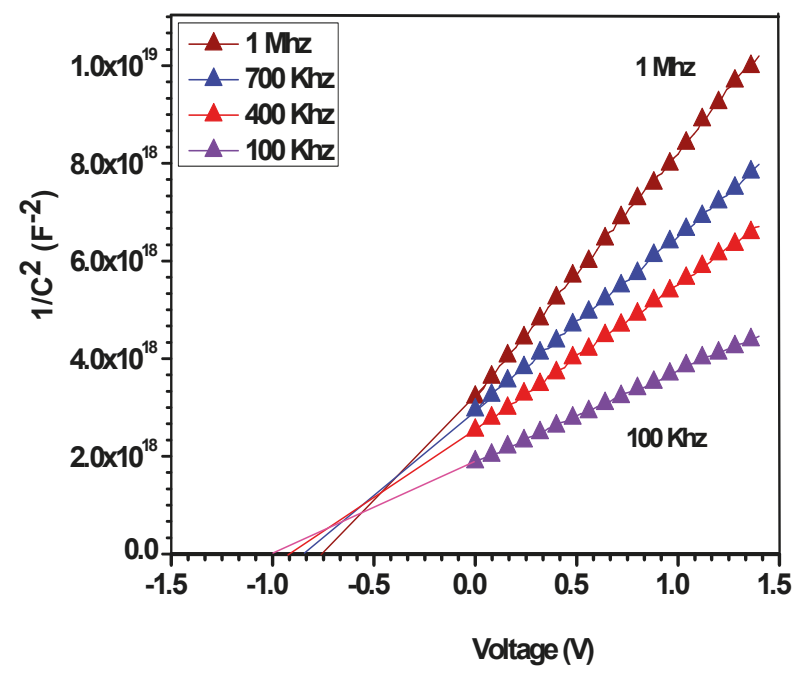

Figure 7. Plot of the $1 / \mathrm{C}^{-2}-V$ for the as-deposited $\mathrm{Al} / \mathrm{SiO}_{2} / p-\mathrm{GaN}$ MOS Schottky diode measurement at different frequencies between $100 \mathrm{kHz}$ and $1 \mathrm{MHz}, \mathrm{AC}=100 \mathrm{mV}$.

Based on Equation (8), the hole concentration of the diode increased from $3.92 \times 10^{16} \mathrm{~cm}^{-3}$ to $5.36 \times 10^{16} \mathrm{~cm}^{-3}$ tested at $100 \mathrm{KHz}$ to $1 \mathrm{MHz}$, while the Fermi level of the diode slightly decreased from 0.109 to $0.099 \mathrm{eV}$. After calculating the values of $V_{0}, V_{b i}, E_{F}, E_{m}$, and $\Delta \Phi_{b}$, based on Equations (9)-(14), the values of $V_{0}$ and the $\mathrm{SBH}$ values were reduced from 0.99 to $0.75 \mathrm{eV}$ and 1.06 to $0.88 \mathrm{eV}$, respectively, when the testing frequencies were changed from $100 \mathrm{kHz}$ to $1 \mathrm{MHz}$ (Table 2). 
Table 2. The parameters calculated from $1 / \mathrm{C}^{2}-V$ for characteristics of the $\mathrm{Al} / \mathrm{SiO}_{2} / p-\mathrm{GaN}$ MOS Schottky diode between $100 \mathrm{kHz}$ and $1 \mathrm{MHz}$ at the room temperature.

\begin{tabular}{cccccc}
\hline $\begin{array}{c}\text { Frequency } \\
(\mathbf{K H z})\end{array}$ & $\boldsymbol{N}_{\boldsymbol{p}}\left(\mathbf{c m}^{-3}\right)$ & $\boldsymbol{E}_{\boldsymbol{F}} \mathbf{( e V )}$ & $\boldsymbol{V}_{\mathbf{0}}(\mathbf{e V})$ & $\boldsymbol{\Delta} \boldsymbol{\Phi}_{\mathbf{b}}(\mathbf{e V})$ & $\boldsymbol{\phi}_{\mathbf{C V}}(\mathbf{e V})$ \\
\hline 1000 & $3.92 \times 10^{16}$ & 0.109 & 0.75 & 0.039 & 0.88 \\
700 & $4.20 \times 10^{16}$ & 0.106 & 0.82 & 0.042 & 0.94 \\
400 & $4.89 \times 10^{16}$ & 0.103 & 0.90 & 0.044 & 1.02 \\
100 & $5.36 \times 10^{16}$ & 0.099 & 0.99 & 0.046 & 1.06 \\
\hline
\end{tabular}

\section{Discussion}

Tables 1 and 2 display all the parameters of the MOS Schottky diode measured by $I-V$ and $C-V$ measurements. This MOS Schottky diode showed an acceptable leakage current of $\sim 4.49 \times 10^{-8} \mathrm{~A}$ at $-1 \mathrm{~V}$, a breakdown voltage of $-6 \mathrm{~V}$, and a turn-on voltage of $\sim 2.1 \mathrm{~V}$. Calculating using Cheung's method, the MOS Schottky had $R_{S}$ of $5918 \Omega$ and $n$ of 3.51. In addition, from the plot of the $I-V$ curve, the ideality factor $n$ was found to be 3.32. This indicated that a higher turn-on voltage leads to a higher ideality factor $[4,9,13]$. The growth of an insulator $\mathrm{SiO}_{2}$ layer can effect to the accumulation layer during the forward bias, which affected to the high value of $R_{s}$ in our Schotky diode. For similar results, by measurement at RT, the series resistance $\left(R_{s}\right)$ and ideality factor $(n)$ were calculated to be $84.4 \mathrm{k} \Omega$ and 2.96 for the $\mathrm{Pt} / \mathrm{SiO}_{2} / n$-GaN MOS Schottky diode [6], $230 \Omega$ and 1.6 for the $\mathrm{Au} / \mathrm{SiO}_{2} / n-\mathrm{GaN}$ MOS diode [13], and $66 \Omega$ and 2.48 for the $\mathrm{Al} / \mathrm{SnO}_{2} / p$-Si (111) MOS diode, respectively [18].

From Table 2, we showed that the $C-V$ measurement data depended strongly on the tested frequency at the RT. At the high frequency $(1 \mathrm{MHz})$, the interface state density could not identify the value of capacitance because it balanced with the semiconductor. At the low frequency, the interface state's density easily followed the AC signal. This created a signal and extra capacitance $[11,13,18]$. The $N_{p}$ and SBH decreased from $5.36 \times 10^{16} \mathrm{~cm}^{3}$ to $3.92 \times 10^{16} \mathrm{~cm}^{3}$ and from 1.06 to $0.88 \mathrm{eV}$, respectively, with increased frequency, due to the existence of the interfacial $\mathrm{SiO}_{2}$ layer in the depletion region. Similarly, the $N_{p}$ of our Schottky diode was $5.36 \times 10^{16} \mathrm{~cm}^{-3}$ when tested at a frequency of $1 \mathrm{MHz}$ at RT [7]. The $N_{p}$ and $\mathrm{SBH}$ of the $\mathrm{Au} / \mathrm{SiO}_{2} / n-\mathrm{GaN}$ MOS diode were $2.08 \times 10^{17} \mathrm{~cm}^{-3}$ and $0.99 \mathrm{eV}$, also tested at a frequency of $1 \mathrm{MHz}$ [9].

The hole's concentration $\left(N_{p}\right)$, calculated from the $1 / C^{2}-V$ plots for the MOS Schottky diode, was lower than that calculated by the Hall measurement because this result was measured from the $p$-Mg-GaN film deposited on the $\mathrm{SiO}_{2} / \mathrm{Si}$ substrate. In addition, with the fast growth rate in deposition, the interface between $p$-GaN and $\mathrm{SiO}_{2}$ affected the polarized $\mathrm{SiO}_{2}$ layer. The electrical field across the depletion region changed significantly near the $p-\mathrm{GaN}$ layer. It was affected by the strong variation in the hole's concentration, leakage current, and turn-on and breakdown voltages of the diode $[11,15,16]$.

Therefore, the SBH of our MOS Schottky diode, in terms of $C-V$ measurement, was higher than the $I-V$ test because of the charges induced by dielectric layer. The $\mathrm{SBH}$ of our diode corresponded with some results $(0.67-1.06 \mathrm{eV})$ of the GaN Schottky diodes made by metal organic chemical vapor deposition (MOCVD) and other approaches. Cheng et al. also reported the SBH of a Pt-oxide- $\mathrm{Al}_{0.3} \mathrm{Ga}_{0.7} \mathrm{As}$ MOS diode, which decreased from 1.03 to $0.86 \mathrm{eV}$ after annealing in a hydrogen atmosphere [16]. Baris et al. reported all the parameters of $\mathrm{Au} / \mathrm{TiO} / n$-Si (100) MOS diodes; an ideality factor of 3.72 and an $\mathrm{SBH}$ of $0.62 \mathrm{eV}$ were determined by testing the $I-V$ measurement. Meanwhile, via calculation by $C-V$ measurement, the SBH and bulk concentration were determined to be $0.99 \mathrm{eV}$ and $9.82 \times 10^{14} \mathrm{~cm}^{-3}$, respectively [23].

\section{Conclusions}

The modeling and electrical properties of the $\mathrm{Al} / \mathrm{SiO}_{2} / p-\mathrm{GaN}$ MOS Schottky diode were successfully established by total RF sputtering. All the parameters were calculated based on $I-V$ and $C-V$ measurements. The SBHs of the MOS Schottky diodes were determined to be $0.67(I-V), 0.78 \mathrm{eV}$ (Norde), and $0.88 \mathrm{eV}(\mathrm{C}-V)$. The hole's concentration, tested by $C-V$ measurement, decreased slightly 
compared with that determined by the Hall measurement. This was due to the existence of the $\mathrm{SiO}_{2}$ layer in the $p$-GaN MOS diode. Our work using cost-effective RF sputtering to make the $\mathrm{Al} / \mathrm{SiO}_{2} / p-\mathrm{GaN}$ MOS Schottky diode can be applied to the development of electronic devices.

Author Contributions: Data curation, P.T.C. and T.A.T.T.; methodology, writing-original draft, investigation, P.T.C. and T.A.T.T., formal analysis, funding acquisition, writing-review and editing, P.T.C., T.A.T.T., P.Q.-P., V.K.N. and N.P.L.T.; supervision, D.-H.K.

Funding: This research was funded by the Ministry of Science and Technology of the Republic of China under grant number 107-2221-E-011-141-MY3.

Conflicts of Interest: The authors declare no conflict of interest.

\section{References}

1. Arslan, E.; Bütüna, S.; Safak, Y.; Özbaya, E.; Uslu, H. Electrical characterization of MS and MIS structures on AlGaN/AlN/GaN heterostructures. Microelectron. Reliab. 2011, 51, 370-375. [CrossRef]

2. Chang, P.C.; Yu, C.L.; Chiu, Y.J.; Liu, C.H.; Chang, S.J.; Su, Y.K.; Chuang, R.W. Ir/Pt Schottky contact oxidation for nitride-based Schottky barrier diodes. Phys. Status Solidi C 2007, 5, 1625-1628. [CrossRef]

3. Li, C.C.; Kuo, D.H. Effects of growth temperature on electrical and structural properties of sputtered GaN films with a cermet target. J. Mater. Sci. Mater. Electron. 2014, 25, 1404-1409. [CrossRef]

4. Reddy, M.S.; Reddy, V.R.; Choi, C.J. Electrical properties and interfacial reactions of rapidly annealed Ni/Ru Schottky rectifiers on $n$-type GaN. J. Alloys. Comp. 2010, 503, 186-191. [CrossRef]

5. Reddy, R.; Rao, P.K. ;Annealing temperature effect on electrical and structural properties of $\mathrm{Cu} / \mathrm{Au}$ Schottky contacts to $n$-type GaN. Microelectron. Eng. 2008, 85, 470-476. [CrossRef]

6. Tuan, T.T.A.; Kuo, D.H.; Li, C.C.; Yen, W.C. Schottky barrier characteristics of Pt contacts to all sputtering-made n-type GaN and MOS diodes. J. Mater. Sci. Mater. Electron. 2014, 25, 3264-3270. [CrossRef]

7. Bengi, S.; Bülbül, M.M. Electrical and dielectric properties of $\mathrm{Al} / \mathrm{HfO}_{2} / \mathrm{p}-\mathrm{Si} \mathrm{MOS}$ device at high temperatures. Curr. Appl. Phys. 2013, 25, 1819-1825. [CrossRef]

8. Tsevas, S.; Vasilopoulou, M.; Kouvatsos, D.N.; Speliotis, T.; Niarchos, D. Characteristics of MOS diodes fabricated using sputter-deposited $\mathrm{W}$ or Cu/W film. Microelectron. Eng. 2006, 83, 1434-1437. [CrossRef]

9. Reddy, V.R.; Reddy, M.S.; Lakshmi, B.P.; Kumar, A. Electrical characterization of Au/n-GaN metal-semiconductor and $\mathrm{Au} / \mathrm{SiO}_{2} / n-\mathrm{GaN}$ metal-insulator-semiconductor structures. J. Alloys. Comp. 2011, 509, 8001-8007. [CrossRef]

10. Lin, K.W.; Chen, H.; Cheng, C.C.; Chuang, H.-M.; Lu, C.T.; Liu, W.C. Characteristics of a new $\mathrm{Pt} /$ oxide/ $\mathrm{In}_{0.49} \mathrm{Ga}_{0.51} \mathrm{P}$ hydrogen-sensing Schottky diode. Sens. Act. B 2003, 94, 145-151. [CrossRef]

11. Tuan, T.T.A.; Kuo, D.H. Characteristics of RF reactive sputter-deposited $\mathrm{Pt} / \mathrm{SiO}_{2} / n$-InGaN MOS Schottky diodes. Mater. Sci. Semicond. Process. 2015, 30,314-320. [CrossRef]

12. Liu, I.P.; Chang, C.H.; Huang, J.; Lin, K.W. Hydrogen sensing characteristics of a Pd/Nickel oxide/GaN-based Schottky diode. Int. J. Hydrogen Energy 2019, 44, 5748-5754. [CrossRef]

13. Lakshmi, B.P.; Reddy, M.S.; Kumar, A.A.; Reddy, V.R. Electrical transport properties of $\mathrm{Au} / \mathrm{SiO}_{2} / n-\mathrm{GaN}$ MIS structure in a wide temperature range. Curr. Appl. Phys. 2012, 12, 765-772. [CrossRef]

14. Juang, F.R.; Fang, Y.K.; Chiu, H.Y. Dependence of the Au/SnO $x / n$-LTPS/glass thin film MOS Schottky diode CO gas sensing performances on operating temperature. Microelectron. Reliab. 2012, 32, 160-165. [CrossRef]

15. Tsai, T.H.; Huang, J.R.; Lin, K.W.; Hsu, W.C.; Chen, H.I.; Liu, W.C. Improved hydrogen sensing characteristics of a Pt $/ \mathrm{SiO}_{2} / \mathrm{GaN}$ Schottky diode. Sens. Act. B 2008, 129, 292-302. [CrossRef]

16. Cheng, C.C.; Tsai, Y.Y.; Lin, K.W.; Chen, H.I.; Lu, C.T.; Liu, W.C. Hydrogen sensing characteristics of a Pt-oxide- $\mathrm{Al}_{0.3} \mathrm{Ga}_{0.7} \mathrm{As}$ MOS Schottky diode. Sens. Act. B 2004, 99, 292-302. [CrossRef]

17. Chen, H.I.; Chang, C.C.; Lu, H.H.; Liu, I.-P.; Chen, W.C.; Ke, B.Y.; Liu, W.C. Hydrogen sensing performance of a Pd/HfO $/ \mathrm{HaN}_{2}$ metal-oxide-semiconductor (MOS) Schottky diode. Sens. Act B 2018, 262, 852-859. [CrossRef]

18. Karadeniz, S.; Tuğluoğlu, N.; Serin, T. Substrate temperature dependence of series resistance in $\mathrm{Al} / \mathrm{SnO} \mathrm{O}_{2} / \mathrm{p}-\mathrm{Si}$ (111) Schottky diodes prepared by spray deposition method. Appl. Surf. Sci. 2004, 233, 5-13. [CrossRef]

19. Chang, C.F.; STai, T.H.; Chen, H.I.; Lin, K.W.; Chen, T.P.; Chen, L.Y.; Liu, Y.C.; Liu, W.C. Hydrogen sensing properties of a Pd/SiO $/$ AlGaN-based MOS diode. Electrochem. Communi. 2009, 11, 65-67. [CrossRef] 
20. Li, C.C.; Kuo, D.H. Material and technology developments of the totally sputtering-made $p-n$ GaN diodes for cost-effective power electronics. J. Mater. Sci. Mater. Electron. 2014, 25, 1942-1948. [CrossRef]

21. Tuan, T.T.A.; Kuo, D.H.; Li, C.C.; Li, G.Z. Effect of temperature dependence on electrical characterization of $p-n$ GaN diode fabricated by RF magnetron sputtering. Mater. Sci. Appl. 2015, 6, 809-817.

22. Ramesh, C.K.; Reddy, V.R.; Choi, C.J. Electrical characteristics of molybdenum Schottky contacts on $n$-type GaN. Mater. Sci. Eng. B 2004, 112, 30-33. [CrossRef]

23. Barış, B. Analysis of device parameters for $\mathrm{Au} / \mathrm{TiO}_{2} / n-\mathrm{Si}$ (100) metal-oxide semiconductor (MOS) diodes. Phys. B 2014, 438, 65-69. [CrossRef]

24. Cheung, S.K.; Cheung, N.W. Extraction of Schottky diode parameters from forward current-voltage characteristics. Appl. Phys. Lett. 1986, 49, 85-87. [CrossRef]

25. Tuan, T.T.A.; Kuo, D.H.; Saragih, A.D.; Li, G.Z. Electrical properties of RF-sputtered Zn-doped GaN films and $p-\mathrm{Zn}-\mathrm{GaN} / n-\mathrm{Si}$ hetero junction diode with low leakage current of $10^{9} \mathrm{~A}$ and a high rectification ratio above $10^{5}$. Mater. Sci. Eng. B 2017, 222, 18-25. [CrossRef]

26. Ravinandan, M.; Rao, P.K.; Reddy, V.R. Temperature dependence of current-voltage $I-V$ characteristics of $\mathrm{Pt} / \mathrm{Au}$ Schottky contacts on n-type GaN. J. Optoelectron. Adv. Mater. 2008, 10, 2787-2792.

27. Norde, H. A modified forward IV plot for Schottky diodes with high series resistance. J. Appl. Phys. 1979, 50, 5052-5053. [CrossRef]

28. Tuan, T.T.A.; Kuo, D.H. Temperature-dependent electrical propertiesof the sputtering-made $n$-InGaN/p-GaN junction diode with a breakdown voltage above 20 V. Mater. Sci. Semicond. Process. 2015, 32, 160-165. [CrossRef]

(C) 2019 by the authors. Licensee MDPI, Basel, Switzerland. This article is an open access article distributed under the terms and conditions of the Creative Commons Attribution (CC BY) license (http://creativecommons.org/licenses/by/4.0/). 
Article

\title{
Electrical Characterization of RF Reactive Sputtered $p-\mathrm{Mg}-\operatorname{In}_{x} \mathrm{Ga}_{1-x} \mathrm{~N} / n-\mathrm{Si}$ Hetero-Junction Diodes without Using Buffer Layer
}

\author{
Thi Tran Anh Tuan ${ }^{1}$, Dong-Hau Kuo ${ }^{2, *}$, Phuong Thao Cao ${ }^{3, *}$, Van Sau Nguyen ${ }^{1}$, \\ Quoc-Phong Pham ${ }^{3}$, Vinh Khanh Nghi $^{3}$ and Nguyen Phuong Lan Tran ${ }^{4}$ \\ 1 School of Basic Sciences, Tra Vinh University, Tra Vinh 87000, Vietnam; thitrananhtuan@tvu.edu.vn (T.T.A.T.); \\ nvsau@tvu.edu.vn (V.S.N.) \\ 2 Department of Materials Science and Engineering, National Taiwan University of Science and Technology, \\ Taipei 10607, Taiwan \\ 3 School of Engineering and Technology, Tra Vinh University, Tra Vinh 87000, Vietnam; \\ phongpham@tvu.edu.vn (Q.-P.P.); nghivinhkhanh@tvu.edu.vn (V.K.N.) \\ 4 College of Engineering and Technology, Can Tho University, Can Tho 94000, Vietnam; tnplan@ctu.edu.vn \\ * Correspondence: dhkuo@mail.ntust.edu.tw (D.-H.K.); cpthao@tvu.edu.vn (P.T.C.); \\ Tel.: +886-2-27303291 (D.-H.K.)
}

Received: 30 August 2019; Accepted: 21 October 2019; Published: 25 October 2019

\begin{abstract}
The modeling of $p-\mathrm{In}_{x} \mathrm{Ga}_{1-x} \mathrm{~N} / n-\mathrm{Si}$ hetero junction diodes without using the buffer layer were investigated with the "top-top" electrode. The $p-\mathrm{Mg}-\mathrm{GaN}$ and $p-\mathrm{Mg}-\mathrm{In}_{0.05} \mathrm{Ga}_{0.95} \mathrm{~N}$ were deposited directly on the $n-\mathrm{Si}(100)$ wafer by the $\mathrm{RF}$ reactive sputtering at $400^{\circ} \mathrm{C}$ with single cermet targets. $\mathrm{Al}$ and Pt with the square size of $1 \mathrm{~mm}^{2}$ were used for electrodes of $p-\mathrm{In}_{x} \mathrm{Ga}_{1-x} \mathrm{~N} / n-\mathrm{Si}$ diodes. Both devices had been designed to prove the $p$-type performance of $10 \% \mathrm{Mg}$-doped in $\mathrm{GaN}$ and InGaN films. By Hall measurement at the room temperature (RT), the holes concentration and mobility were determined to be $N_{p}=3.45 \times 10^{16} \mathrm{~cm}^{-3}$ and $\mu=145 \mathrm{~cm}^{2} / \mathrm{V}$.s for $p-\mathrm{GaN}$ film, $N_{p}=2.53 \times 10^{17} \mathrm{~cm}^{-3}$, and $\mu=45 \mathrm{~cm}^{2} / \mathrm{V}$.s for $p-\mathrm{InGaN}$ film. By the $I-V$ measurement at RT, the leakage currents at $-5 \mathrm{~V}$ and turn-on voltages were found to be $9.31 \times 10^{-7} \mathrm{~A}$ and $2.4 \mathrm{~V}$ for $p-\mathrm{GaN} / n-\mathrm{Si}$ and $3.38 \times 10^{-6} \mathrm{~A}$ and $1.5 \mathrm{~V}$ for $p-\mathrm{InGaN} / n-\mathrm{Si}$ diode. The current densities at the forward bias of $20 \mathrm{~V}$ were 0.421 and $0.814 \mathrm{~A} \cdot \mathrm{cm}^{-2}$ for $p-\mathrm{GaN} / n-\mathrm{Si}$ and $p-\mathrm{InGaN} / n-\mathrm{Si}$ devices. The electrical properties were measured at the temperature range of 25 to $150^{\circ} \mathrm{C}$. By calculating based on the TE mode, Cheungs' and Norde methods, and other parameters of diodes were also determined and compared.
\end{abstract}

Keywords: $p$-Mg-InGaN films; RF sputtering; I- $V$ measurement; Cheung's method; Norde's method; TE mode

\section{Introduction}

$\mathrm{GaN}$ and InGaN have excellent characteristics such as high conductivity and high mobility. The development and creation of $p$-layer $\mathrm{GaN}$ and InGaN materials involve one of the important technologies in designing electronic devices [1-5]. The investigation of high-quality doping in GaN and InGaN semiconductors by incorporating elements such as $\mathrm{Zn}$, and $\mathrm{Cu}, \mathrm{Mg}$ for $p-\mathrm{GaN}$ behavior, and its alloys had reported [4-8]. The success of $\mathrm{Mg}$ doping in forming $p-\mathrm{In}_{x} \mathrm{Ga}_{1-x} \mathrm{~N}$ films is an important factor for developing electric devices, a photo detector, and solar cell devices [7-10]. Si wafer has often been used for the growth of $\mathrm{GaN}$, InGaN, and their alloys for applications in photo-detector, solar cells, and electronic devices. The combination between $n-\mathrm{InGaN}$ layers and $n-\mathrm{Si}$ wafers were studied to improve the interface layers by using an assortment of approaches [11-14]. For fabricated electronic devices, the growth of the InGaN layer on $n-S i$ wafers were studied to improve the interface layers by using a variety of approaches [15-20]. Lee at al. studied electrical properties of a nanowire $n-\mathrm{GaN} / p-\mathrm{Si}$ 
device by forming dielectrophoretic alignment. At the current density of $10-60 \mathrm{~A} / \mathrm{cm}^{2}$, the diode was well-defined with a forward voltage drop of 1.2-2.0 V and high resistance in the range of $447 \mathrm{~K} \Omega$ [17]. $\mathrm{Li}$ et al. used the RF sputtering method to deposit the Mg-doped GaN films on the Si substrate and design the homo junction $\mathrm{GaN}$ diodes. By testing the $I-V$ measurement, the turn-on voltages of diodes were 2.3 and $2.1 \mathrm{~V}$ for as-deposited and $500{ }^{\circ} \mathrm{C}$-annealed sample, respectively [21]. Vinay Kabra et al. investigated the $p-\mathrm{ZnO} / n-\mathrm{Si}$ hetero junction diode by using a dip coating technique. Their electrical properties showed the highly rectifying, with a rectification ratio of 101 at $3 \mathrm{~V}$ [22]. Mohd Yusoff et al. reported the $p-n$ junction diode based on GaN grown on the AlN/Si (111) substrate and annealed samples at $700{ }^{\circ} \mathrm{C}$. The ideality factors of their diodes decreased from 19.68 to 15.14 , with the testing temperature increasing from 30 to $104{ }^{\circ} \mathrm{C}$ [23]. In previous work, the $\mathrm{Mg}$-doped $\operatorname{In}_{x} \mathrm{Ga}_{1-x} \mathrm{~N}$ films had been deposited on $\mathrm{Si}(100)$ substrates by RF reactive sputtering. The $\mathrm{Mg}-\mathrm{In}_{x} \mathrm{Ga}_{1-x} \mathrm{~N}$ films had the $p$-type conduction at $x \leq 0.075$. The $p-\mathrm{Mg}-\mathrm{In}_{0.05} \mathrm{Ga}_{0.95} \mathrm{~N} / n-\mathrm{GaN}$ diode was shown the leakage current of $2.7 \times 10^{-6} \mathrm{~A}$, turn-on voltage of $1.8 \mathrm{~V}$, and breakdown voltage of $6.8 \mathrm{~V}$ at the RT [12].

All previous groups often investigated the $p-\mathrm{GaN}$ films and their alloys by using MOCVD above $800{ }^{\circ} \mathrm{C}$ and other methods. The sputtered technique with the low temperature at and below $400{ }^{\circ} \mathrm{C}$ has been hardly declared. Furthermore, there is no report on the electrical properties of $p-\mathrm{Mg}-\mathrm{In}_{x} \mathrm{Ga}_{1-x} \mathrm{~N} / n-\mathrm{Si}$ diodes without using buffer layers. In this study, to prove the success of $\mathrm{Mg}$ doping in the $p-\mathrm{GaN}$ and the $p-\operatorname{InGaN}$ films, the modeling of $p-\mathrm{Mg}-\mathrm{In}_{x} \mathrm{Ga}_{1-x} \mathrm{~N} / n-\mathrm{Si}$ hetero junction diodes was designed by using the RF reactive sputtering. This method was chosen to design diodes due to the benefits of low sputtered-temperature, low cost, and safe working atmosphere $[7,13,15]$. The $n-\mathrm{Si}$ (100) wafer was also used for its low cost, large wafer size, and easy availability $[18,22,24]$. The electrical characteristics of devices were calculated by the thermionic emission (TE) mode at different testing temperatures $[3,12,13]$.

\section{Materials and Methods}

Figure 1 shows the structural modeling of a $p-\mathrm{In}_{x} \mathrm{Ga}_{1-x} \mathrm{~N} / n-\mathrm{Si}$ hetero junction diode. This device was designed on the $n-\mathrm{Si}(100)$ wafer by modeling with the "top-top" electrode. The $p-\mathrm{Mg}-\mathrm{In}_{0.05} \mathrm{Ga}_{0.95} \mathrm{~N}$ and $p-\mathrm{Mg}-\mathrm{GaN}$ films were deposited together on the $n-\mathrm{Si}(100)$ and $\mathrm{SiO}_{2} / \mathrm{Si}(100)$ substrate. The $n-\mathrm{Si}(100)$ wafer had sheet resistance of $\sim 1-10 \Omega \cdot \mathrm{cm}$, diameter of 2 inches, thickness of $\sim 550 \mu \mathrm{m}$, and the polished surface. Sputtered- $\mathrm{In}_{x} \mathrm{Ga}_{1-x} \mathrm{~N}$ films on $\mathrm{SiO}_{2} / \mathrm{Si}$ substrate were used for testing Hall measurement and SEM analysis.

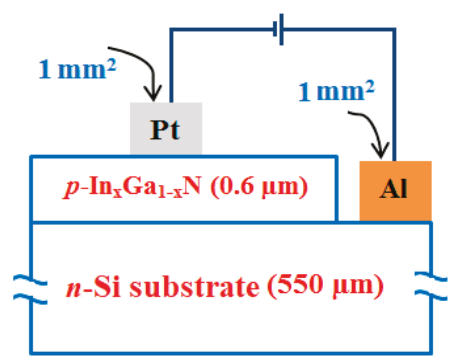

Figure 1. Structural modeling of $p-\operatorname{In}_{x} \mathrm{Ga}_{1-x} \mathrm{~N} / n-\mathrm{Si}$ hetero junction diode.

For the $p-\mathrm{GaN}$ film, the sputtering cermet target was made by hot pressing with the mixture of metallic $\mathrm{Mg}$, Ga powders, and $\mathrm{GaN}$ powder. The $[\mathrm{Mg}] /([\mathrm{Ga}]+[\mathrm{Mg}])$ molar ratio in each cermet target was reserved at $10 \%$. Similar for $p-\operatorname{InGaN}$ film, the $[\mathrm{Mg}] /([\mathrm{In}]+[\mathrm{Ga}]+[\mathrm{Mg}])$ molar ratio in each cermet target was also kept at $10 \%$. The $[\mathrm{In}] /([\mathrm{In}]+[\mathrm{Ga}]+[\mathrm{Mg}])$ molar ratios was $5 \%$. The $p-\mathrm{Mg}-\mathrm{In}_{x} \mathrm{Ga}_{1-x} \mathrm{~N}$ films (with $x \sim 0$ and 0.05) were deposited on $n-\mathrm{Si}(100)$ substrates at $400^{\circ} \mathrm{C}$ for $25 \mathrm{~min}$ by RF reactive sputtering. Both $\mathrm{Mg}-\mathrm{In}_{x} \mathrm{Ga}_{1-x} \mathrm{~N}$ targets were sputtered with $\mathrm{RF}$ power of $150 \mathrm{~W}$ under the gas mixture of $\mathrm{Ar}$ and $\mathrm{N}_{2}$, which remained at $5 \mathrm{sccm}$ for each. 
The pure Aluminum (99.99\%) and Platinum (99.99\%) targets were used for making the electrodes for $p-\operatorname{In}_{x} \mathrm{Ga}_{1-x} \mathrm{~N} / n-\mathrm{Si}$ hetero junction diodes. By using steel masks with the square size of $1 \mathrm{~mm}^{2}$, these electrodes were deposited at $200{ }^{\circ} \mathrm{C}$, with RF power of $80 \mathrm{~W}$ for 30 min on top of $p-\operatorname{In}_{x} \mathrm{Ga}_{1-x} \mathrm{~N}$ films and $n-\mathrm{Si}$ substrate. The detail procedure for creating the $p-\mathrm{GaN}$ and $p-\mathrm{InGaN}$ films, made by RF reactive sputtering, can be referred to our previous works [10-12,21].

In this work, our diodes were designed at the low sputtered-temperature and high pressure. The holes' concentration, electrical conductivities, and mobilities of $p-\mathrm{Mg}-\operatorname{In}_{x} \mathrm{Ga}_{1-x} \mathrm{~N}$ films and $n-\mathrm{Si}$ wafer were measured by a Hall measurement (HMS-2000, Ecopia, Tokyo, Japan). Scanning electron microscopy (SEM, JSM-6500F, JEOL, Tokyo, Japan) was used to observe the surface morphology of $p-\mathrm{In}_{x} \mathrm{Ga}_{1-x} \mathrm{~N}$ films. Energy dispersive spectrometer (EDS, JSM-6500F) equipped on SEM was used for composition analysis of films. The $I-V$ characteristics of $p-\mathrm{In}_{x} \mathrm{Ga}_{1-x} \mathrm{~N} / n-\mathrm{Si}$ diodes were measured by using Semiconductor Device Analyzer (Agilent, B1500A, Tokyo, Japan) with the different temperature. All parameters of diodes were calculated by following the equations of the TE mode as well as the Cheungs' and Norde method.

\section{Results and Discussion}

\subsection{Structural and Electrical Characteristics}

The carrier concentration $\left(N_{p}\right)$ and carrier mobility $(\mu)$ of $n-S i$ wafer were found to be $N_{\mathrm{p}}=4.7 \times 10^{15} \mathrm{~cm}^{-3}$ and $=196 \mathrm{~cm}^{2} /$ V.s when measuring the Hall effect at room temperature (RT). The holes concentration $\left(N_{\mathrm{p}}\right)$ and mobility $(\mu)$ were also determined to be $N_{p}=3.45 \times 10^{16} \mathrm{~cm}^{-3}$, $\mu=145 \mathrm{~cm}^{2} / \mathrm{V}$.s for $p-G a N$ film, and $N_{\mathrm{p}}=2.53 \times 10^{17} \mathrm{~cm}^{-3}, \mu=45 \mathrm{~cm}^{2} / \mathrm{V} \cdot \mathrm{s}$ for $p-\operatorname{InGaN}$ film, respectively. By SEM analysis, the thicknesses of Pt and Al layers were $\sim 250 \mathrm{~nm}$ and the thickness of both $p-\operatorname{In}_{x} \mathrm{Ga}_{1-x} \mathrm{~N}$ films was $\sim 0.6 \mu \mathrm{m}$.

\subsection{The Energy Band Diagram}

Figure 2 displays the energy band diagram of a $p-\operatorname{In}_{0.5} \mathrm{Ga}_{0.95} \mathrm{~N} / n-\mathrm{Si}$ diode as an example. Our band gap $\left(E_{\mathrm{g}}\right)$ of $\mathrm{Mg}$-doped $\operatorname{In}_{0.5} \mathrm{Ga}_{0.95} \mathrm{~N}\left(\mathrm{Mg}\right.$ of $10 \%$ ) films at $400{ }^{\circ} \mathrm{C}$-deposition were $2.92 \mathrm{eV}$. The $E_{\mathrm{g}}$ was reduced by increasing In content in $\operatorname{In}_{x} \mathrm{Ga}_{1-x} \mathrm{~N}$ [10,21]. In a similar result, Wagner et al. reported the composition dependence of the band gap energy of the strained $\operatorname{In}_{x} \mathrm{Ga}_{1-x} \mathrm{~N}$ layers grown by MOCVD. The energy $E_{\mathrm{g}}$ of $\operatorname{In}_{x} \mathrm{Ga}_{1-x} \mathrm{~N}$ decreased from 3.43 to $3.28 \mathrm{eV}$ with the increasing in ratio from 0.02 to 0.15 [25]. The band gap values of $n-\mathrm{Si}$ was also found to be $1.12 \mathrm{eV}$. The electron affinities $\chi$ of $p-\mathrm{InGaN}$ and $n-\mathrm{Si}$ were $4.20 \mathrm{eV}$ and $4.05 \mathrm{eV}$, respectively [6,24]. According to the band gap values of $p-\mathrm{In}_{0.5} \mathrm{Ga}_{0.95} \mathrm{~N}$ and $n-\mathrm{Si}\left(2.92\right.$ and $1.12 \mathrm{eV}$ ), the barrier $\Delta E_{\mathrm{c}}$ for electrons was followed by $\Delta E_{\mathrm{c}}=\chi_{p-\mathrm{InGaN}}-\chi_{\mathrm{Si}}$ and the barrier $\Delta E_{\mathrm{v}}$ for holes was calculated by $\Delta \mathrm{E}_{\mathrm{v}}=E_{\mathrm{g}(\mathrm{InGaN})}-E_{\mathrm{g}(\mathrm{Si})}+\Delta E_{\mathrm{c}}$ (Figure 2). As a result, the energy band diagram showed a small conduction band offset of $0.15 \mathrm{eV}$ and a large valance band offset of $1.95 \mathrm{eV}$ [22,24-26]. Therefore, the electrons' injection from $n-\mathrm{Si}$ into $p-\operatorname{In}_{x} \mathrm{Ga}_{1-x} \mathrm{~N}$ will be easier than the holes' injection from $p-\mathrm{In}_{x} \mathrm{Ga}_{1-x} \mathrm{~N}$ into $n-\mathrm{Si}$ because the energetic barrier $\Delta E_{\mathrm{v}}$ of holes is many times higher than the barrier $\Delta E_{\mathrm{c}}$ of electrons. With the existing space charge layer at the forward bias, the current is limited. However, the depletion width will decrease because of the low potential barrier, and the current will increase easily by increasing the voltage $[6,27,28]$.

\subsection{I-V Measurements}

Figure 3 shows the current density-voltage characteristics of $p-\mathrm{GaN} / n-\mathrm{Si}$ (device-A) and $p-$ InGaN/n-Si (device-B) tested at RT based on a $1-\mathrm{mm}^{2}$ contact. The applied voltage of measurement was extended from $-20 \mathrm{~V}$ to $+20 \mathrm{~V}$ for both devices. The current densities and the leakage current densities are found in the area of $1 \mathrm{~cm}^{2}$. 


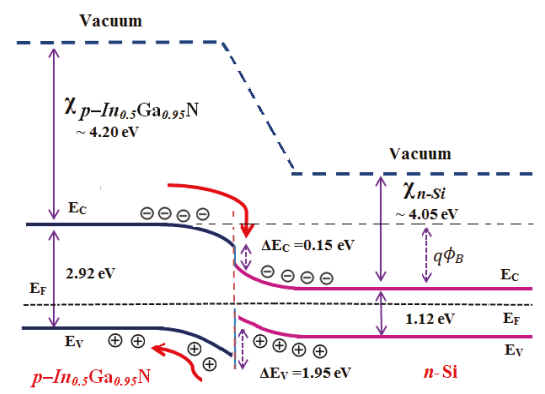

Figure 2. Energy band diagram of the $p-I n_{0.5} \mathrm{Ga}_{0.95} \mathrm{~N} / n-\mathrm{Si}$ hetero junction diode.

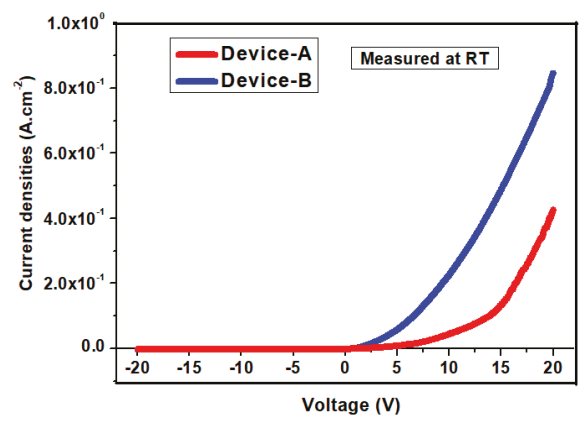

Figure 3. Current-voltage characteristics of $p-\mathrm{GaN} / n-\mathrm{Si}$ and $p-\mathrm{InGaN} / n$-Si diode, measured at room temperature.

At the bias range of $(-5 \mathrm{~V},+5 \mathrm{~V})$, the leakage current of diodes at $-1 \mathrm{~V}$ and the turn-on voltages at RT were $1.43 \times 10^{-7} \mathrm{~A}, 1.5 \mathrm{~V}$ (device-A) and $2.58 \times 10^{-7} \mathrm{~A}, 2.4 \mathrm{~V}$ (device-B). Our devices had an improvement in the turn-on voltage with the increase in content in the $p$-InGaN layer.

With the wide voltage of $(-20 \mathrm{~V},+20 \mathrm{~V})$, Figure 3 also shows the breakdown voltage was beyond the largest instrument capacity of $\sim 20 \mathrm{~V}$ for both diodes. The leakage currents also were found to be $9.31 \times 10^{-7}$ and $3.38 \times 10^{-6} \mathrm{~A}$ at $-5 \mathrm{~V}$ for (device-A) and (device-B), respectively. At the forward bias of $20 \mathrm{~V}$, the current densities of diodes were 0.421 and $0.814 \mathrm{~A} \cdot \mathrm{cm}^{-2}$ for device-A and device-B, respectively. Higher holes concentration in the $p$-InGaN layer with the performance of the in ratio in $p$-InGaN leads to the higher current density of device-B. In addition, with the high conduction of electrodes, the forward current of diodes increased rapidly at the bias of $(-20 \mathrm{~V},+20 \mathrm{~V})$. The rectification ratio (on/off) of diodes were also found to be $3.22 \times 10^{4}$ (device-A) and $6.61 \times 10^{4}$ (device-B), respectively.

Figure 4 shows the semilogarithmic $I-V$ characteristics of diodes under the forward bias, which were tested at the RT temperature. All parameters of diodes can be calculated by the thermionic-emission (TE) mode (for $q V>3 \mathrm{kT}$ ). They are given by the equations below $[3,11,12]$.

$$
\begin{gathered}
I=I_{0}\left[\exp \frac{q}{n k T}\left(V-I R_{\mathrm{s}}\right)\right] \\
\phi_{\mathrm{b}}=\frac{k T}{q} \ln \left(\frac{A A^{*} T^{2}}{I_{0}}\right) \\
n=\frac{q}{k T}\left(\frac{\mathrm{d} V}{\mathrm{~d} \ln I}\right) \\
A^{*}=\frac{4 \pi q k^{2} m^{*}}{h^{3}}
\end{gathered}
$$


where $A^{*}$ is the effective Richardson constant, $R_{\mathrm{S}}$ is the series resistance, $n$ is the ideality factor, $I_{0}$ is the saturation current, $q$ is the electronic charge, $A$ is the area of diode, $\phi_{\mathrm{b}}$ is the barrier height, $m_{\mathrm{e}}$ is the free electron mass, $m^{*}$ is the effective electron mass, and $h$ is the Plank constant [29-31]. In base Equation (4), the theoretical value of $A^{*}$ was $26.4 \mathrm{~A} \cdot \mathrm{cm}^{-2} \cdot \mathrm{K}^{-2}$ for $p-\mathrm{GaN}\left(m^{*}=0.22 m_{\mathcal{e}}\right)$, and $23 \mathrm{~A} \cdot \mathrm{cm}^{-2} \cdot \mathrm{K}^{-2}$ for $p$-InGaN $\left(m^{*}=0.19 m_{\mathrm{e}}\right)[6,12,13]$.

As shown in Figure 4, the barrier height of diodes can be calculated from $I_{0}$ as the saturation current. Based on Equation (2), and from the plot of $\ln I$ versus $V$, the saturation current $I_{\mathrm{O}}$ can be determined by intersecting the interpolated straight lines from the linear region with the current axis $[12,20]$.

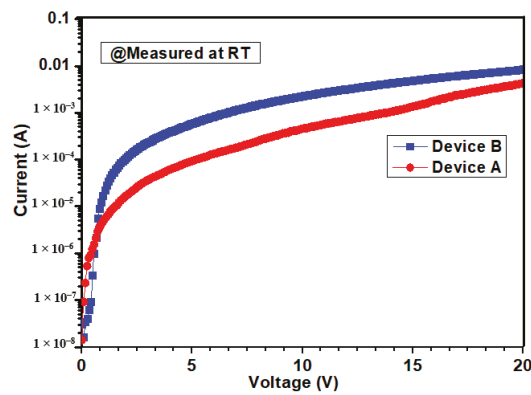

Figure 4. The $I-V$ characteristics of device-A and device-A under the forward bias tested at room temperature.

Figure $5 \mathrm{a}, \mathrm{b}$ show the semi logarithmic $I-V$ characteristics of diodes were tested at the temperature range from 25 to $150^{\circ} \mathrm{C}$. From Figure 5, the leakage current of diode at $-5 \mathrm{~V}$ increased from $9.39 \times 10^{-7} \mathrm{~A}$ (device-A) and $3.38 \times 10^{-6}$ (device-B) at $25^{\circ} \mathrm{C}$ to $3.25 \times 10^{-5} \mathrm{~A}$ (device-A) and $2.64 \times 10^{-4} \mathrm{~A}$ (device-B) at $150{ }^{\circ} \mathrm{C}$. Based upon Equations (1)-(3), the barrier height values were found to increase from 0.54 to $0.69 \mathrm{eV}$ (device-A), and 0.50 to $0.62 \mathrm{eV}$ (device-B), whereas the ideality factor $n$ decreased from 5.9 to 4.8 (device-A), and 5.1 to 3.6 (devices-B) with testing temperatures ranging from 25 to $150{ }^{\circ} \mathrm{C}$. These achieved parameters of diodes made by RF sputtering are similar to some previous works with the GaN-diodes made by MOCVD and techniques [1,3,18,28].

Similar to the analysis of the parameters for Schottky diodes, the effect of series resistance $R_{\mathrm{S}}$ will influence and change the ideality factor $n$. They can be determined by using Cheung's method. It is given by the equation below $[13,32,33]$.

$$
\frac{\mathrm{d} V}{\mathrm{~d}(\ln I)}=\frac{n k T}{q}+I R_{\mathrm{S}}
$$

Based on Equation (5) and the plot of the $\mathrm{d} V / \mathrm{d}(\ln I)$ versus $I$ from Figure 6, the series resistance $R_{s}$ and ideality $n$ can be calculated the from the the slope and the intercept $[11,33,34]$. Table 1 shows all detailed parameters of the $I-V$ measurement for both devices tested at different temperatures. With the high Indium content, the $R_{S}$ and $n$ values of device-B were smaller than device-A. They showed $412 \Omega$ and 5.3 at $25^{\circ} \mathrm{C}$ and decreased them to $133 \Omega$ and 3.8 at $150{ }^{\circ} \mathrm{C}$.

By comparison, with Cheung's method, the modified Norde function was also used to define the effective barrier height of diodes. It is followed by the equation below [12,13,34-36].

$$
F(V, I)=\frac{V}{\gamma}-\frac{k T}{q} \ln \left(\frac{I}{A A^{*} T^{2}}\right)
$$

The effective barrier height $\phi_{\mathrm{B}}$ is followed by the equation below.

$$
\phi_{\mathrm{B}}=F\left(V_{\min }\right)+\frac{V_{\min }}{\gamma}-\frac{k T}{q}
$$


where the $\mathrm{F}\left(V_{\min }\right)$ is the minimum value of $\mathrm{F}(V, I), \gamma$ is the first integer (dimensionless) greater than $n$, and $V_{\min }$ is the corresponding voltage at $\mathrm{F}\left(V_{\min }\right)$.

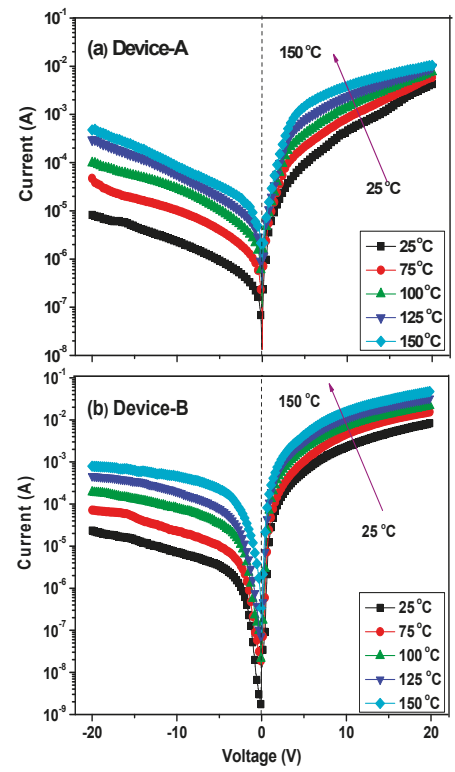

Figure 5. Forward and reverse $(I-V)$ characteristics of (a) device-A and (b) device-B tested in the temperature range of $25-150{ }^{\circ} \mathrm{C}$.
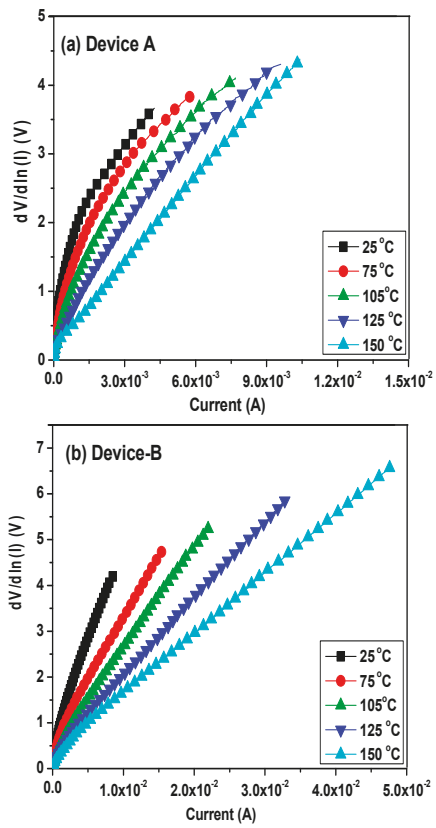

Figure 6. Plots of $\mathrm{d} V / \mathrm{d} \ln (I)$ versus $I$ for (a) device-A and (b) device-B tested in the temperature range of 25 to $150{ }^{\circ} \mathrm{C}$. 
Figure 7a,b shows the plot of $\mathrm{F}(V, I)$ versus $V$ of hetero junction diodes as a function of the different temperatures tested. By using Equations (6) and (7), the barrier height values were determined to be $0.56 \mathrm{eV}$ (device-A) and $0.53 \mathrm{eV}$ (device-B) at $25^{\circ} \mathrm{C}$, and reached $0.71 \mathrm{eV}$ (device A) and $0.64 \mathrm{eV}$ (device B) at $150{ }^{\circ} \mathrm{C}$ (Table 1$)$.
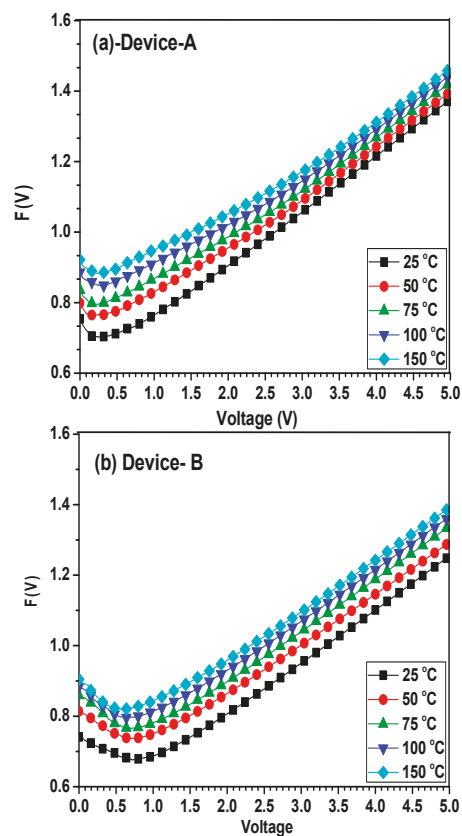

Figure 7. The $F(V)$ versus $V$ plot for (a) device-A and (b) device-B tested in the temperature range of 25 to $150{ }^{\circ} \mathrm{C}$.

\section{Discussion}

Table 1 showed the detailed comparisons of the $I-V$ measurements for both hetero junction diodes. The smallest leakage current of $9.31 \times 10^{-7} \mathrm{~A}$ at $-5 \mathrm{~V}$ was found in device-A tested at RT, while the smallest turn-on voltage was $\sim 1.5 \mathrm{~V}$. The highest current density of $0.814 \mathrm{~A} \cdot \mathrm{cm}^{-2}$ were found in device-B at the forward bias of $20 \mathrm{~V}$. At the reverse bias, the breakdown voltage of both devices was strongly blocked with the value at $-20 \mathrm{~V}$. The forward current increased quickly with the occurrence of the in content in the $p$-InGaN layer. With a higher holes carrier concentration of $2.53 \times 10^{17} \mathrm{~cm}^{-3}$ and a smaller band gap of $2.92 \mathrm{eV}$, device-B showed the small turn-on voltage of $1.5 \mathrm{~V}$, barrier heights of $0.5 \mathrm{eV}$ (Cheung's method), and $0.53 \mathrm{eV}$ (Norde method), compared with all parameters of device-A [20].

The ideality factor $n$ of diodes decreased quickly, from 5.9 at $25^{\circ} \mathrm{C}$ for device-A to 3.6 at $150{ }^{\circ} \mathrm{C}$ for device-B. For the Schottky diode, the turn-on voltage is often small, and the ideality factor $n$ is $<2[2,3,13]$. In our diodes, the ideality factors had high values because of the present high series' resistances, which were calculated from the slope of the linear region in Cheung's method. In addition, from the band diagram in Figure 2, the barrier $\Delta E_{\mathrm{v}}$ for holes was larger than that of $\Delta E_{\mathrm{c}}$. Therefore, a higher valance band offset will have a high potential barrier, large turn-on voltage, high ideality factor, and high series resistance Rs. The high ideality factor of our diodes at RT also has been attributed to the high structural defect density, which serves as the trap-assisted generation-recombination centers. This procedure also influences the current transportation of hetero junction diodes $[13,17,20]$. The $R_{\mathrm{S}}$ values also reduced from $876 \Omega$ at $25^{\circ} \mathrm{C}$ (device-A) to $133 \Omega$ at $150{ }^{\circ} \mathrm{C}$ (device-B). The generations of 
series resistance, interface states, and the voltage drop across the interfacial layer caused the slight difference between the ideality factor $n$ calculated from the $\ln I$ vs $V$ and $\mathrm{d} V / \mathrm{d}(\ln I)$ vs. $I$ plots (Table 1$)$.

Both hetero junction diodes had remarkable improvements as compared with some other homo and hetero junction diodes made by different techniques. Their deposition had epitaxial growth and was conducted at high temperatures above $800{ }^{\circ} \mathrm{C}$ by $\operatorname{MOVCD}[18,19,27,37-39]$. Our devices had displayed the special $I-V$ characteristics, which can be attributed to the low-temperature deposited at $-400{ }^{\circ} \mathrm{C}$ without using a buffer layer. Both our diodes showed the breakdown voltage above $20 \mathrm{~V}$ for a $1 \mathrm{~mm}^{2}$-sized contact.

Table 1. The parameters calculated from electrical characteristics of hetero junction diodes as a function of testing temperatures.

\begin{tabular}{|c|c|c|c|c|c|c|}
\hline \multirow{2}{*}{$\operatorname{Temp}\left({ }^{\circ} \mathrm{C}\right)$} & \multirow{2}{*}{$\begin{array}{c}\text { Leakage Current (A) } \\
\text { at }-5 \mathrm{~V}\end{array}$} & \multicolumn{2}{|c|}{ Barrier Height $\phi_{B}(\mathrm{eV})$} & \multirow{2}{*}{$\begin{array}{c}\text { From } I-V \\
n\end{array}$} & \multicolumn{2}{|c|}{ Cheung's Function $\mathrm{d} V / \mathrm{d} \ln (I)$ versus $I$} \\
\hline & & $I-V$ & Norde & & $R_{\mathrm{s}}(\Omega)$ & $n$ \\
\hline \multicolumn{7}{|l|}{ Device-A } \\
\hline 25 & $9.31 \times 10^{-7}$ & 0.54 & 0.56 & 5.9 & 876 & 6.4 \\
\hline 75 & $4.02 \times 10^{-6}$ & 0.57 & 0.58 & 5.6 & 683 & 5.8 \\
\hline 100 & $1.03 \times 10^{-5}$ & 0.63 & 0.64 & 5.4 & 549 & 5.5 \\
\hline 125 & $1.91 \times 10^{-5}$ & 0.66 & 0.68 & 5.0 & 467 & 5.3 \\
\hline 150 & $3.26 \times 10^{-5}$ & 0.69 & 0.71 & 4.8 & 413 & 5.1 \\
\hline \multicolumn{7}{|l|}{ Device-B } \\
\hline 25 & $3.38 \times 10^{-6}$ & 0.50 & 0.53 & 5.1 & 486 & 5.3 \\
\hline 75 & $9.92 \times 10^{-5}$ & 0.53 & 0.55 & 4.8 & 292 & 4.9 \\
\hline 100 & $3.43 \times 10^{-5}$ & 0.57 & 0.60 & 4.6 & 233 & 4.7 \\
\hline 125 & $7.81 \times 10^{-5}$ & 0.60 & 0.62 & 4.1 & 173 & 4.2 \\
\hline 150 & $2.64 \times 10^{-4}$ & 0.62 & 0.64 & 3.6 & 133 & 3.8 \\
\hline
\end{tabular}

K.E.F. Keskenler et al. made the $n-\mathrm{ZnO} / p-\mathrm{Si}$ hetero junction diode by using a sol-gel spin technique [38]. The leakage current, barrier height, ideality factor, and series resistance of their diode were determined to be $4 \times 10^{-6} \mathrm{~A}$ at $-1 \mathrm{~V}, 0.71 \mathrm{eV}, 2.03$, and $42.1 \Omega$ at RT. Chirakkara et al. investigated the $n-\mathrm{ZnO} / p-\mathrm{Si}$ (100) diode by pulsed laser deposition without using a buffer layer [39]. By $I-V$ testing at the temperature range of 300-390 K, the barrier height increased from $0.6(300 \mathrm{~K})$ to $0.76 \mathrm{eV}$ ( $390 \mathrm{~K})$. Seng et al. reported the $n-\mathrm{ZnO} / p-\mathrm{GaN}$ diode formed by the RF technique [40]. Their diode had showed the turn-on voltage of $2 \mathrm{~V}$, the leakage current of $1.6 \times 10^{-5} \mathrm{~A}$, and the series resistances of $102 \Omega$. Hsueh et al. investigated the $n-\mathrm{Mg}_{x} \mathrm{Zn}_{1-x} \mathrm{O} / p-\mathrm{GaN}$ hetero-junction diode. Their ideality factors decreased from 3.86 to 7.00 , with testing temperature increasing from 25 to $125^{\circ} \mathrm{C}$ [41]. Compared to our previous works, for the sputtering-made $n-\operatorname{In}_{x} \mathrm{Ga}_{1-x} \mathrm{~N} / p-\mathrm{Si}$ devices with the similar area of $1-\mathrm{mm}^{2}$, the leakage current densities at $-5 \mathrm{~V}$ were $5.96 \times 10^{-5} \mathrm{~A} \cdot \mathrm{cm}^{-2}$ for $n-\mathrm{GaN} / p-\mathrm{Si}$ and $2.81 \times 10^{-4} \mathrm{~A} \cdot \mathrm{cm}^{-2}$ for $n-\operatorname{In}_{0.4} \mathrm{Ga}_{0.6} \mathrm{~N} / p-\mathrm{Si}$, respectively [20].

At this time, there is rarely a report about the $\mathrm{Mg}$-doped- $\mathrm{In}_{x} \mathrm{Ga}_{1-x} \mathrm{~N}$ film made by RF sputtering below $400{ }^{\circ} \mathrm{C}$ deposition and designed diode on the $n-\mathrm{Si}$ wafer. With the improvement of doping $\mathrm{Mg}$ in $\mathrm{In}_{x} \mathrm{Ga}_{1-x} \mathrm{~N}$ film, our devices had the stable $I-V$ characteristics up to the testing temperature of $150{ }^{\circ} \mathrm{C}$. The $p-\operatorname{In}_{x} \mathrm{Ga}_{1-x} \mathrm{~N} / n-$ Si diodes with the strong breakdown voltage of $20 \mathrm{~V}$ and leakage current of $\sim 10^{-7}$ A will create the opportunity to develop the power devices.

\section{Conclusions}

$p-\operatorname{In}_{x} \mathrm{Ga}_{1-x} \mathrm{~N} / n-\mathrm{Si}$ diodes were successfully investigated by directly depositing the $p$-GaN and $p-$ Mg-doped- $\operatorname{In}_{0.5} \mathrm{Ga}_{0.95} \mathrm{~N}$ films on $n$-Si(100) wafers without using a buffer layer. The highest current density of $0.814 \mathrm{~A} \cdot \mathrm{cm}^{-2}$ at $20 \mathrm{~V}$ and the smallest turn-on voltage of $1.5 \mathrm{~V}$ were found for $p-\operatorname{InGaN} / n-\mathrm{Si}$ devices. Pt and $\mathrm{Al}$ for Omhic contacts contributed to the high current density at the forward bias of hetero junction diodes. Both diodes also displayed the good $I-V$ characteristics up to the testing temperature of $150{ }^{\circ} \mathrm{C}$ and the breakdown voltages of $20 \mathrm{~V}$. By calculating the equations based on the TE mode as well as Cheungs' and Norde method, the obtained electrical parameters were also compared. They can be referred to develop cost-effective solutions in electronic devices. 
Author Contributions: Data Curation, P.T.C. and T.T.A.T.; Methodology, Writing-Original Draft, Investigation, P.T.C. and T.T.A.T.; Formal Analysis, Funding Acquisition, Writing-Review and Editing, P.T.C., T.T.A.T., V.S.N., Q.-P.P., V.K.N. and N.P.L.T.; Supervision, D.-H.K

Funding: This research was funded by the Ministry of Science and Technology of the Republic of China under grant number 107-2221-E-011-141-MY3.

Conflicts of Interest: The authors declare no conflict of interest.

\section{References}

1. Baik, K.H.; Irokawa, Y.; Ren, F.; Pearton, S.J.; Park, S.S.; Park, S.J. Temperature dependence of forward current characteristics of GaN junction and Schottky rectifiers. Solid State Electron. 2003, 47, 1533-1538. [CrossRef]

2. Jang, J.S.; Kim, D.; Seong, T.Y. Schottky barrier characteristics of Pt contacts to n-type InGaN. J. Appl. Phys. 2006, 99, 073704. [CrossRef]

3. Reddy, V.R.; Prasanna, B.P.; Padma, R. Electrical Properties of Rapidly Annealed Ir and Ir/Au Schottky Contacts on n-Type InGaN. J. Metall. 2012, 1, 1-9. [CrossRef]

4. Cao, X.A.; Lachode, J.R.; Ren, F. Implanted p-n junction in GaN. Solid State Electron. 1999, 43, 1235-1238. [CrossRef]

5. Hickman, R.; Vanhove, J.M.; Chow, P.P.; Klaassen, J.J. GaN PN junction issues and developments. Solid State Electron. 2000, 44, 377-381. [CrossRef]

6. Tuan, T.T.A.; Kuo, D.H.; Albert, D.S.; Li, G.Z. Electrical properties of RF-sputtered Zn-doped GaN films and $\mathrm{p}-\mathrm{Zn}-\mathrm{GaN} / \mathrm{n}$-Si hetero junction diode with low leakage current of $10^{-9} \mathrm{~A}$ and a high rectification ratio above 105. Mater. Sci. Eng. B 2017, 222, 18-25. [CrossRef]

7. Islam, M.R.; Sugita, K.; Horie, M.; Islam, A.Y. Mg doping behavior of MOVPE $\operatorname{In}_{x} \mathrm{Ga}_{1-x} \mathrm{~N}(x \sim 0.4)$. J.Cryst. Growth 2009, 311, 2817-2820. [CrossRef]

8. Yohannes, K.; Kuo, D.H. Growth of p-type Cu-doped GaN films with magnetron sputtering at and below $400{ }^{\circ}$ C. Mater. Sci. Semicond. Process. 2015, 29, 288-293.

9. Ager, J.W.; Miller, N.; Jones, R.E. Mg-doped InN and InGaN Photoluminescence, Capacitance-Voltage and thermo-power measurements. Phys. Status Solidi 2008, 245, 873-877. [CrossRef]

10. Kuo, D.H.; Li, C.C.; Tuan, T.T.A.; Yen, C.L. Effects of Mg Doping on the Performance of InGaN Films Made by Reactive Sputtering. J. Electron. Mater. 2014, 44, 210-216. [CrossRef]

11. Tuan, T.T.A.; Kuo, D.H.; Li, C.C.; Li, G.Z. Effect of temperature dependence on electrical characterization of p-n GaN diode fabricated by RF magnetron sputtering. Mater. Sci. Appl. 2015, 6, 809-817.

12. Tuan, T.T.A.; Kuo, D.H.; Li, C.C.; Yen, W.L. Electrical and structural properties of Mg-doped $\operatorname{In}_{x} \mathrm{Ga}_{1-x} \mathrm{~N}(x \leq 0.1)$ and p-InGaN/n-GaN junction diode made all by RF reactive sputtering. Mater. Sci. Eng. B 2015, 193, 13-19.

13. Tuan, T.T.A.; Kuo, D.H.; Li, C.C.; Yen, W.L. Schottky barrier characteristics of Pt contacts to all sputtering-made n-type GaN and MOS diodes. Mater. Sci. Mater. Electron. 2014, 25, 3264-3270. [CrossRef]

14. Oh, M.; Lee, J.J.; Lee, L.K.; Oh, H.K. Electrical characteristics of Mg-doped p-GaN treated with the electrochemical potentiostatic activation method. J. Alloy Compd. 2014, 585, 414-417. [CrossRef]

15. Umeno, M.; Egawa, T.; Ishikawa., H. GaN-based optoelectronic devices on sapphire and Si substrates. Mater. Sci. Semicond. Process. 2001, 4, 459-466. [CrossRef]

16. Liou, B.W. Design and fabrication of $\operatorname{In}_{x} \mathrm{Ga}_{1-x} \mathrm{~N} / \mathrm{GaN}$ solar cells with a multiple-quantum-well structure on SiCN/Si(111) substrates. Thin Solid Films 2011, 520, 1084-1090. [CrossRef]

17. Lee, S.Y.; Kim, T.H.; Suh, D.I.; Park, J.E. An electrical characterization of a hetero-junction nanowire (NW) PN diode (n-GaN NW/pSi) formed by dielectrophoresis alignment. Phys. E 2007, 36, 194-198. [CrossRef]

18. Zebbar, N.; Kheireddine, Y.; Mokeddema, K.; Hafdallah, A.; Kechouanea, M.; Aida, M.S. Structural optical and electrical properties of $\mathrm{n}-\mathrm{ZnO} / \mathrm{p}-\mathrm{Si}$ heterojunction prepared by ultrasonic spray. Mater. Sci. Semicond. Process. 2011, 14, 229-234. [CrossRef]

19. Gad, A.E.; Hoffmann, M.W.G.; Hernandez, R.F. Coaxial p-Si/n-ZnO nanowire heterostructures for energy and sensing applications. Mater. Chem. Phys. 2012, 135, 618-622. [CrossRef]

20. Tuan, T.T.A.; Kuo, D.H.; Lin, K.; Li, G.Z. Temperature dependence of electrical characteristics of $n$ - $\operatorname{In}_{x} \mathrm{Ga}_{1-x} \mathrm{~N} /$ p-Si hetero-junctions made totally by RF magnetron sputtering. Thin Solid Films 2015, 589, 182-187. [CrossRef]

21. Li, C.C.; Kuo, D.H. Material and technology developments of the totally sputtering-made p/n GaN diodes for cost-effective power electronics. Mater. Sci. Mater. Electron. 2014, 25, 1942-1948. [CrossRef] 
22. Kabra, V.; Aamir, L.; Malik, M. Low cost, p-ZnO/n-Si, rectifying nano heterojunction diode: Fabrication and electrical characterization. Beilstein J. Nanotechnol. 2014, 5, 2216-2221. [CrossRef] [PubMed]

23. Mohd, M.Z.; Baharin, A.; Hassan, Z.; Abu, H.; Abdullah, M.J. MBE growth of GaN pn-junction photodetector on AlN/Si substrate with Ni/Ag as Ohmic contact. Superlattices Microstruct. 2013, 56, 35-44. [CrossRef]

24. Cho, S.G.; Nahm, T.U.; Kim, E.K. Deep level states and negative photoconductivity in n-ZnO/p-Si hetero-junction diodes. Appl. Phys. 2014, 14, 223-226. [CrossRef]

25. Wagner, J.; A. Ramakrishnan, A.; Behr, D.; Maier, M. Composition dependence of the band gap energy of $\mathrm{In}_{x} \mathrm{Ga}_{1-x} \mathrm{~N}$ layers on $\mathrm{GaN}(x \leq 0.15)$ grown by metal-organic chemical vapor deposition. MRS. Internet. J. Nitri. Semi. Res. 1999, 4, 106-111. [CrossRef]

26. Bedia, F.Z.; Bedia, A.; Benyoucef, B.; Hamzaoui, S. Electrical characterization of n-ZnO/p-Si heterojunction prepared by spray pyrolysis technique. Phys. Procedia 2014, 55, 61-67. [CrossRef]

27. Baydogan, N.; Karacasu, O.; Cimenoglu, H. Effect of annealing temperature on $\mathrm{ZnO}: \mathrm{Al} / \mathrm{p}-\mathrm{Si}$ heterojunctions. Thin Solid Films 2012, 520, 5790-5796. [CrossRef]

28. Li, J.L.; Schubert, E.F.; Graff, J.W.; Osinsky, A.; Schaff, W.F. Low-resistance ohmic contacts to p-type GaN. Appl. Phys. Lett. 2000, 76, 2728-2730. [CrossRef]

29. Ponce, A.R.; Olguín, D.; Calderón, H.I. Calculation of the effective masses of II-VI semiconductor compounds. Superficies y Vacío 2003, 16, 26-28.

30. Suzuki, M.; Uenoyama, T. First-Principles Calculation of Effective Mass Parameters of Gallium Nitride. Jpn. J. Appl. Phys. 1995, 34, 3442-3446. [CrossRef]

31. Crowell, C.R. The richardson constant for thermionic Emission in schottky barrier diodes. Solid State Electron. 1965, 8, 395-399. [CrossRef]

32. Kumar, A.; Vinayak, S.; Singh, R. Micro-structural and temperature dependent electrical characterization of Ni/GaN Schottky barrier diodes. Curr. Appl. Phys. 2013, 13, 1137-1142. [CrossRef]

33. Cheung, S.K.; Cheung, N.W. Extraction of Schottky diode parameters from forward current-voltage characteristics. Appl. Phys. Lett. 1986, 49, 85-87. [CrossRef]

34. Benamara, Z.; Akkal, B.; Talbi, A.; Gruzza, B. Electrical transport characteristics of Au/n-GaN Schottky diodes. Mater. Sci. Eng. C 2006, 26, 519-522. [CrossRef]

35. Tuan, T.T.A.; Kuo, D.H. Characteristics of RF reactive sputter-deposited $\mathrm{Pt} / \mathrm{SiO}_{2} / \mathrm{n}-\mathrm{InGaN}$ MOS Schottky diodes. Mater. Sci. Semicond. Process. 2015, 30,314-320. [CrossRef]

36. Norde, H. A modified forward I-V plot for Schottky diodes with high series resistance. J. Appl. Phys. 1979, 50, 5052-5505. [CrossRef]

37. Urgessa, Z.N.; Dobson, S.R.; Talla, K.; Murape, D.M. Optical and electrical characteristics of ZnO/Si heterojunction. Phys. B 2014, 439, 149-152. [CrossRef]

38. Keskenler, E.F.; Tomakina, M.; Dogan, S. Growth and characterization of Ag/n-ZnO/p-Si/Al heterojunction diode by sol-gel spin technique. J. Alloy Compd. 2013, 550, 129-132. [CrossRef]

39. Chirakkara, S.; Krupanidhi, S.B. Study of n-ZnO/p-Si (100) thin film heterojunctions by pulsed laser deposition without buffer layer. Thin Solid Films 2012, 520, 5894-5899. [CrossRef]

40. Shen, Y.; Chen, X.; Yan, X.S. Low-voltage blue light emission from n-ZnO/p-GaN heterojunction formed by RF magnetron sputtering method. Curr. Appl. Phys. 2014, 14, 345-348. [CrossRef]

41. Hsueh, K.P. Temperature dependent current-voltage characteristics of $\mathrm{n}-\mathrm{Mg}_{x} \mathrm{Zn}_{1-x} \mathrm{O} / \mathrm{p}-\mathrm{GaN}$ junction diodes. Microelectron. Eng. 2011, 88, 1016-1018. [CrossRef]

(C) 2019 by the authors. Licensee MDPI, Basel, Switzerland. This article is an open access article distributed under the terms and conditions of the Creative Commons Attribution (CC BY) license (http://creativecommons.org/licenses/by/4.0/). 
Article

\title{
Structural Properties and Oxidation Resistance of $\mathrm{ZrN} / \mathrm{SiN}_{\mathrm{x}}, \mathrm{CrN} / \mathrm{SiN}_{\mathrm{x}}$ and $\mathrm{AlN} / \mathrm{SiN}_{\mathrm{x}}$ Multilayered Films Deposited by Magnetron Sputtering Technique
}

\author{
Ihar Saladukhin ${ }^{1}$, Gregory Abadias ${ }^{2, *}$, Vladimir Uglov ${ }^{1,3}$, Sergey Zlotski ${ }^{1}$, \\ Arno Janse van Vuuren ${ }^{4}$ and Jacques Herman $\mathrm{O}^{\prime}$ Connell ${ }^{4}$ \\ 1 Faculty of Physics, Belarusian State University, 220030 Minsk, Belarus; solodukhin@bsu.by (I.S.); \\ Uglov@bsu.by (V.U.); Zlotski@bsu.by (S.Z.) \\ 2 Institut Pprime, Université de Poitiers-CNRS-ENSMA, TSA 41123, CEDEX 9, 86073 Poitiers, France \\ 3 South Ural State University, 454080 Chelyabinsk, Russia \\ 4 Centre for HRTEM, Nelson Mandela Metropolitan University, Port Elizabeth 6001, South Africa; \\ arnojvv@gmail.com (A.J.v.V.); joconnell@mandela.ac.za (J.H.O.) \\ * Correspondence: gregory.abadias@univ-poitiers.fr; Tel.: +33-(0)549-496-748
}

Received: 24 December 2019; Accepted: 4 February 2020; Published: 7 February 2020

\begin{abstract}
In the present work, the structure, stress state and phase composition of $\mathrm{MeN} / \mathrm{SiN}_{\mathrm{x}}(\mathrm{Me}=\mathrm{Zr}$, $\mathrm{Cr}, \mathrm{Al})$ multilayered films with the thickness of elementary layers in nanoscale range, as well as their stability to high temperature oxidation, were studied. Monolithic (reference) and multilayered films were deposited on Si substrates at the temperatures of $300{ }^{\circ} \mathrm{C}\left(\mathrm{ZrN} / \mathrm{SiN}_{\mathrm{x}}\right.$ and $\mathrm{AlN} / \mathrm{SiN}_{\mathrm{x}}$ systems $)$ or 450 ${ }^{\circ} \mathrm{C}\left(\mathrm{CrN} / \mathrm{SiN}_{\mathrm{x}}\right)$ by reactive magnetron sputtering. The thickness ratios of $\mathrm{MeN}$ to $\mathrm{SiN}_{\mathrm{x}}$ were $5 \mathrm{~nm} / 2 \mathrm{~nm}$, $5 \mathrm{~nm} / 5 \mathrm{~nm}, 5 \mathrm{~nm} / 10 \mathrm{~nm}$ and $2 \mathrm{~nm} / 5 \mathrm{~nm}$. Transmission electron microscopy (TEM), X-ray Reflectivity (XRR) and X-ray Diffraction (XRD) testified to the uniform alternation of $\mathrm{MeN}$ and $\mathrm{SiN}_{\mathrm{x}}$ layers with sharp interlayer boundaries. It was observed that $\mathrm{MeN}$ sublayers have a nanocrystalline structure with (001) preferred orientation at $5 \mathrm{~nm}$, but are X-ray amorphous at $2 \mathrm{~nm}$, while $\mathrm{SiN}_{\mathrm{x}}$ sublayers are always X-ray amorphous. The stability of the coatings to oxidation was investigated by in situ XRD analysis (at the temperature range of $400-950^{\circ} \mathrm{C}$ ) along with the methods of wavelength-dispersive $\mathrm{X}$-ray spectroscopy (WDS) and scanning electron microscopy (SEM) after air annealing procedure. Reference $\mathrm{ZrN}$ and $\mathrm{CrN}$ films started to oxidize at the temperatures of 550 and $700{ }^{\circ} \mathrm{C}$, respectively, while the AlN reference film was thermally stable up to $950^{\circ} \mathrm{C}$. Compared to reference monolithic films, $\mathrm{MeN} / \mathrm{SiN}_{\mathrm{x}}$ multilayers have an improved oxidation resistance (onset of oxidation is shifted by more than $200^{\circ} \mathrm{C}$ ), and the performance is enhanced with increasing fraction of $\mathrm{SiN}_{\mathrm{x}}$ layer thickness. Overall, $\mathrm{CrN} / \mathrm{SiN}_{\mathrm{x}}$ and $\mathrm{AlN} / \mathrm{SiN}_{\mathrm{x}}$ multilayered films are characterized by noticeably higher resistance to oxidation as compared to $\mathrm{ZrN} / \mathrm{SiN}_{\mathrm{x}}$ multilayers, the best performance being obtained for $\mathrm{CrN} / \mathrm{SiN}_{\mathrm{x}}$ and AlN/SiN $\mathrm{S}_{\mathrm{x}}$ with $5 \mathrm{~nm} / 5 \mathrm{~nm}$ and $5 \mathrm{~nm} / 10 \mathrm{~nm}$ periods, which remain stable at least up to $950{ }^{\circ} \mathrm{C}$.
\end{abstract}

Keywords: multilayered film; metal nitride; silicon nitride; oxidation

\section{Introduction}

In accordance with the tendency of industry development, the coatings applied for protection of materials should satisfy more stringent requirements. They have to possess high hardness and wear resistance (e.g., pieces under friction), resistance to high temperature oxidation (e.g., cutting tools) and thermal cyclability (e.g. glass molding dies), stability in corrosive media (e.g., in chemical production units), radiation stability (e.g., materials for nuclear power engineering) and other properties. Currently, physically-vapor deposited transition metal nitride (TMN) coatings based on MeN mononitrides of transition metal $(\mathrm{Me}=\mathrm{Ti}, \mathrm{Zr}$ or $\mathrm{Cr})$ are widely used [1-3]. However, mononitride films often lose 
their protective role in such severe conditions. For example, TiN and $\mathrm{ZrN}$ coatings deposited by reactive magnetron sputtering are intensively oxidized at the temperatures of $500-600{ }^{\circ} \mathrm{C}$ [4-7]. This is related to their columnar microstructure and presence of defects (e.g., porosity or micro-cracks), which allows a direct contact between the external atmosphere and substrate and accelerates oxygen incorporation through grain boundary diffusion. Several routes can be employed to enhance oxidation resistance of MeN coatings. Musil et al. [8-10] showed that superior thermal stability, above $1000{ }^{\circ} \mathrm{C}$, was achieved for hard amorphous coatings, based on either ternary Me-Si-N systems with Si content $\geq$ 20 at. $\%$ or quaternary Si-B-C-N system with covalent bonding. These amorphous coatings are generally obtained by co-sputtering method and their improved oxidation resistance is ascribed to the absence of grain boundaries.

An alternate route to nanocomposite/amorphous Me-Si-N single-layer coatings is to deposit sequentially different $\mathrm{MeN}_{\mathrm{x}}$ layers, resulting in the formation of periodic multilayers or nanolaminates with improved performance characteristics [11]. By alternation at the nanoscale of dissimilar layers, it is possible to combine the advantages of the different materials properties, and even to get superior properties compared to monolithic films such as improved adhesion, increase in corrosion resistance or change in electrical behavior. The authors of [12] showed that, unlike $\mathrm{CrN}$ and $\mathrm{AlN}$ films, for which the generation and motion of classical dislocations is responsible for the plastic flow, the observed plastic deformation in CrN/AlN multilayered coatings is mostly governed by grain rotation for the nanocrystals and grain boundary sliding for grains of larger size. Such non-elastic phenomena prevent the deformation or crack formation that allows using CrN/AlN multilayered coatings for ductile steel substrate as well. Another example is the case of $\mathrm{AlCrN} / \mathrm{TiVN}$ multilayered coatings, where the $\mathrm{AlCrN}$ layer inhibited the excessive oxygen diffusion into the multilayered film [13]. Consequently, it was possible to avoid rapid oxidation of vanadium and obtain high wear-resistance for the AlCrN/TiVN coating. Although the TiVN coating had the lowest friction coefficient, the lowest wear rate $\left(1.9 \times 10^{-7}\right.$ $\mathrm{mm}^{3} / \mathrm{Nm}$ ) was obtained for the AlCrN/TiVN coated sample.

It is possible to classify three main types of multilayers based on the type of structure of the elementary layers: (i) nanocrystalline/nanocrystalline layers; (ii) nanocrystalline/amorphous layers; and (iii) amorphous/amorphous layers.

Multilayers based on TMN usually belong to the first category [12-17]. The comparison of hardness and other mechanical characteristics of multilayered coatings made of alternate stacking of various TMN is given in [15]. It is pointed that the TiN/VN multilayered coatings are one of the most successful examples of superhard materials. The maximum hardness value of these coatings reached $56 \mathrm{GPa}$ (at bilayer thickness about $5.2 \mathrm{~nm}$ ). The use of a combination of nanocrystalline/nanocrystalline layers is rather effective for achievement of high resistance of the coatings to high temperature oxidation $[16,17]$. High thermal stability of $\mathrm{AlCrN} / \mathrm{TiSiN}$ coating is noted, for which the excellent oxidation resistance was obtained with no pore and delamination up to $900{ }^{\circ} \mathrm{C}$ [17]. In this case, the redistribution of elements between the layers takes place when forming the oxide layer, namely mixed ( $\mathrm{Al}, \mathrm{Ti})$-oxide scale outside layer and a dense $(\mathrm{Al}, \mathrm{Cr})_{2} \mathrm{O}_{3}$ inner layer.

The second group of multilayered coatings, consisting of sequentially alternating layers of nanocrystalline and amorphous material, is perspective due to the possibility of combining of the different properties. For example, the hardness and wear resistance of nanocrystalline layer are supplemented with plasticity and chemical inertness of the amorphous layer. $\mathrm{MeN} / \mathrm{SiN}_{\mathrm{x}}$ coatings are among the most appealing nanocrystalline/amorphous layered systems [18-21]. The characteristic feature of these films is the immiscibility of their constitutive layers at the origin of their good thermal stability and properties $[20,22,23]$. The enhanced stability of $\mathrm{MeN} / \mathrm{SiN}_{\mathrm{x}}$ films to oxidation is also related to the fact that the columnar growth of MeN layer is suppressed by the presence of the $\mathrm{SiN}_{\mathrm{x}}$ amorphous layer [21]. This prevents from oxygen penetration deep into the film along the grain boundaries and pores concomitant to the formation of the columnar structure.

A significant increase in hardness of the $\mathrm{MeN} / \mathrm{SiN}_{\mathrm{x}}$ multilayered films has been reported when decreasing the thickness of $\mathrm{SiN}_{\mathrm{x}}$ layer below $\sim 0.8 \mathrm{~nm}$ [23-25]. In this case, the epitaxial growth 
of silicon nitride at the interface of cubic MeN layer results in $\mathrm{SiN}_{\mathrm{x}}$ layer crystallization. Such an effect is more pronounced for $\mathrm{TiN}_{\mathrm{SiN}}$ and $\mathrm{ZrN} / \mathrm{SiN}_{\mathrm{x}}$ systems $[18,24-26]$, and is less marked for the $\mathrm{AlN} / \mathrm{SiN}_{\mathrm{x}}$ system because the interfaces between $\mathrm{AlN}$ and epitaxial $\mathrm{Si}_{3} \mathrm{~N}_{4}$ only slightly affect the propagation of dislocations. As the result, only a minor hardness enhancement is expected in AlN/SiN ${ }_{x}$ multilayers [27]. However, it should be noted that an increase in microhardness of $\mathrm{MeN} / \mathrm{SiN}_{\mathrm{x}}$ multilayered coatings at the thicknesses of $\mathrm{SiN}_{\mathrm{x}}$ elementary layer of $1 \mathrm{~nm}$ and less is accompanied by a decrease in film oxidation resistance [6]. This indicates that, by controlling the ratio of individual layers, it is possible to ensure one or another required property.

Among the coatings of the third group (amorphous/amorphous layers), the coatings with alternating layers of so-called metallic glasses are of more interest for practical applications. $\mathrm{ZrCu} / \mathrm{ZrCuNiAlSi}$ films are an example of radiation-resistant multilayered systems for applications in the area of materials design for nuclear power engineering [28].

In our prior work on the $\mathrm{ZrN} / \mathrm{SiN}_{\mathrm{x}}$ system, we showed that the coatings which belong to the second group are quite promising for use as resistant coatings under high-temperature oxidation up to $860-950{ }^{\circ} \mathrm{C}$ [6]. It should be noted that, for the $\mathrm{CrN} / \mathrm{SiN}_{\mathrm{x}}$ system and, especially, for the $\mathrm{AlN} / \mathrm{SiN}_{\mathrm{x}}$ system, the chemical inertness of $\mathrm{SiN}_{\mathrm{x}}$ amorphous layer is supplemented by the formation of the passivating layers of metal oxide in the surface layer of the $\mathrm{MeN} / \mathrm{SiN}_{\mathrm{x}}$ multilayered film $[17,29]$. However, it is difficult to compare the oxidation behavior of the different $\mathrm{MeN} / \mathrm{SiN}_{\mathrm{x}}$ multilayered systems because of the different physical vapor deposition processes used for their fabrication, as well as variations in the bilayer thickness range studied. In the present work, we performed a systematic and comparative study of the structure and stability to high-temperature oxidation of $\mathrm{ZrN} / \mathrm{SiN}_{\mathrm{x}}, \mathrm{CrN} / \mathrm{SiN}_{\mathrm{x}}$ and $\mathrm{AlN} / \mathrm{SiN}_{\mathrm{x}}$ films sputter-deposited in the same chamber, with special emphasis laid on the influence of the thickness ratio of the elementary layers. As shown previously for the $\mathrm{ZrN} / \mathrm{SiN}_{\mathrm{x}}$ system, the ratio of thicknesses of individual layers plays a key role in their oxidation resistance [6]. The properties of the multilayers are also discussed and compared to those of reference monolithic films.

\section{Materials and Methods}

Reference $\mathrm{ZrN}, \mathrm{CrN}, \mathrm{AlN}$ and $\mathrm{Si}_{3} \mathrm{~N}_{4}$ monolithic films as well as $\mathrm{ZrN} / \mathrm{SiN}{ }_{\mathrm{x}}, \mathrm{CrN} / \mathrm{SiN}_{\mathrm{x}}$ and $\mathrm{AlN} / \mathrm{SiN}_{\mathrm{x}}$ multilayered films were grown by reactive magnetron sputter-deposition in a high vacuum chamber (base pressure $<10^{-5} \mathrm{~Pa}$ ) equipped with confocal targets configuration and a cryogenic pump (max. $500 \mathrm{~L} / \mathrm{s}$ ) [30]. All films were deposited on Si substrates covered with $10 \mathrm{~nm}$ thick thermally grown $\mathrm{SiO}_{2}$ layer (to prevent the interdiffusion of the coating and substrate components). A constant bias voltage of $-60 \mathrm{~V}$ was applied to the substrate during deposition. The substrate was rotated at $15 \mathrm{rpm}$ throughout the deposition to ensure an equal deposition rate across the substrate surface.

Water-cooled, 7.62-cm-diameter Zr (99.2\% purity), Cr (99.95\% purity), Al (99.9995\% purity) and $\mathrm{Si}_{3} \mathrm{~N}_{4}\left(99.99 \%\right.$ purity) targets, located at $18 \mathrm{~cm}$ from the substrate holder, were used under $\mathrm{Ar}+\mathrm{N}_{2}$ plasma discharges at constant power mode. The $\mathrm{Zr}, \mathrm{Cr}$ and $\mathrm{Al}$ targets were operated in magnetically unbalanced configuration using a DC power supply, while for the $\mathrm{Si}_{3} \mathrm{~N}_{4}$ target a RF power supply was used in balanced mode. The total working pressure varied from 0.22-0.29 Pa depending on the material system, as measured using a Baratron ${ }^{\circledR}$ capacitance gauge (Andover, MA, USA). The $\mathrm{Ar} / \mathrm{N}_{2}$ flow ratio was optimized to obtain stoichiometric nitrogen content in the films based on earlier results [31,32] (see Table 1). The $\mathrm{N}_{2}$ partial pressure was measured during deposition using a MKS Microvision mass spectrometer (Munich, Germany).

The deposition conditions were not identical for all MeN layers. They varied $\left(\mathrm{N}_{2}\right.$ partial pressure and substrate temperature) to achieve the desired phase and optimal crystallinity for each MeN layer. Reference $\mathrm{ZrN}$, AlN and $\mathrm{Si}_{3} \mathrm{~N}_{4}$ films as well as $\mathrm{ZrN} / \mathrm{SiN}_{\mathrm{x}}$ and $\mathrm{AlN} / \mathrm{SiN} \mathrm{N}_{\mathrm{x}}$ multilayered films were deposited at $300{ }^{\circ} \mathrm{C}$, while reference $\mathrm{CrN}$ film and $\mathrm{CrN} / \mathrm{SiN}_{\mathrm{x}}$ multilayered films were formed the substrate temperature of $450^{\circ} \mathrm{C}$. This temperature was chosen to obtain single-phase cubic $\mathrm{CrN}$ phase without formation of hexagonal $\mathrm{Cr}_{2} \mathrm{~N}$ phase. The periodic growth of the multilayered systems was 
monitored by computer-controlled pneumatic shutters located at $2 \mathrm{~cm}$ in front of each target. Details of the multilayered films growth procedure are given in [23].

Table 1. Process parameters and chemical analysis for $\mathrm{ZrN}, \mathrm{CrN}, \mathrm{AlN}$ and $\mathrm{Si}_{3} \mathrm{~N}_{4}$ reference films.

\begin{tabular}{cccccccccccc}
\hline Film & $\mathrm{T}_{\text {dep. }}\left({ }^{\circ} \mathrm{C}\right)$ & $\begin{array}{c}\text { Me Power } \\
(\mathbf{W})\end{array}$ & $\begin{array}{c}\mathrm{Si}_{3} \mathbf{N}_{4} \\
\text { Power }(\mathbf{W})\end{array}$ & $\begin{array}{c}\text { Ar/ } \mathbf{N}_{2} \\
\text { Flow }\end{array}$ & $\begin{array}{c}\text { Total } \\
\text { Pressure(Pa) }\end{array}$ & $\begin{array}{c}\text { Partial } \\
\text { Pressure } \\
\boldsymbol{P}_{\boldsymbol{N}_{2}}(\mathbf{P a})\end{array}$ & $\begin{array}{c}\text { Growth } \\
\text { Rate(nm/min) }\end{array}$ & $\begin{array}{c}\text { Film } \\
\text { Thick-ness } \\
\text { (nm) }\end{array}$ & Me (at. \%) & Si (at. \%) & N (at. \%) \\
\hline $\mathrm{ZrN}$ & 300 & 300 & - & $10 / 0.5$ & 0.22 & $4.6 \times 10^{-3}$ & 12.5 & 249 & 46.6 & - \\
$\mathrm{CrN}$ & 450 & 200 & - & $25 / 20$ & 0.29 & $6.3 \times 10^{-2}$ & 5.4 & 259 & 54.6 & - \\
$\mathrm{AlN}$ & 300 & 300 & - & $24 / 6.5$ & 0.22 & $2.4 \times 10^{-2}$ & 5.9 & 293 & 43.6 & - \\
$\mathrm{Si}_{3} \mathrm{~N}_{4}$ & 300 & - & 176 & $24 / 5.1$ & 0.22 & $2.2 \times 10^{-2}$ & 2.1 & 287 & - & 43.4 \\
\hline
\end{tabular}

Note. For $\mathrm{ZrN} / \mathrm{SiN}_{\mathrm{x}}, \mathrm{CrN} / \mathrm{SiN}_{\mathrm{x}}$ and $\mathrm{AlN} / \mathrm{SiN}_{\mathrm{x}}$ films, the same deposition regimes as for $\mathrm{ZrN}, \mathrm{CrN}, \mathrm{AlN}$ and $\mathrm{Si}_{3} \mathrm{~N}$ reference films were used.

The deposition conditions are summarized in Table 1 for the reference monolithic films, and the same conditions were used for elementary layers of the $\mathrm{MeN}_{\mathrm{SiN}}(\mathrm{Me}=\mathrm{Zr}, \mathrm{Cr}, \mathrm{Al})$ multilayers, except the deposition time. The deposition rates were 12.5, 5.9 and $5.4 \mathrm{~nm} / \mathrm{min}$ for $\mathrm{ZrN}$, AlN and CrN monolithic films, respectively. The use of a RF discharge to deposit the $\mathrm{Si}_{3} \mathrm{~N}_{4}$ reference film resulted in a slower deposition rate of $2.1 \mathrm{~nm} / \mathrm{min}$. For mononitrides, the deposition time was adjusted between $22 \mathrm{~min}$ and $2.3 \mathrm{~h}$ to get similar nominal film thickness of $\sim 300 \mathrm{~nm}$. For $\mathrm{MeN} / \mathrm{SiN}_{\mathrm{x}} \mathrm{multilayers}$, the deposition time of constitutive sublayers was adjusted accordingly to get the desired modulation period, but the total film thickness was similar ( 300 nm). For example, for MeN (5 nm)/SiNx (5 nm), the deposition times were $23 \mathrm{~s} / 141 \mathrm{~s}, 50 \mathrm{~s} / 141 \mathrm{~s}$ and $50 \mathrm{~s} / 141 \mathrm{~s}$ for MeN=ZrN, AlN and CrN, respectively. The nominal ratios of thickness of MeN layer to $\mathrm{SiN}_{\mathrm{x}}$ layer were $5 \mathrm{~nm} / 2 \mathrm{~nm}, 5 \mathrm{~nm} / 5 \mathrm{~nm}, 5 \mathrm{~nm} / 10 \mathrm{~nm}$ and $2 \mathrm{~nm} / 5 \mathrm{~nm}$ (see Table 2). For multilayers, the deposition process always started with the MeN sublayer being deposited first.

Table 2. Best-fit parameters as determined from simulations of XRR experimental data of MeN/SiN ${ }_{x}$ $(\mathrm{Me}=\mathrm{Zr}, \mathrm{Cr}, \mathrm{Al})$ multilayers: elementary layer thickness $\left(h_{\mathrm{MeN}}\right.$ and $\left.h_{\mathrm{SiNx}}\right)$, mass density $\left(\rho_{\mathrm{MeN}}\right.$ and $\left.\rho_{\mathrm{SiNx}}\right)$, interface roughness $\left(w_{\mathrm{MeN}}\right.$ and $\left.w_{\mathrm{SiNx}}\right)$, MeN layer fraction $\left(f_{\mathrm{MeN}}\right)$ and total film thickness. The first row indicates nominal thicknesses ratio for $\mathrm{MeN}_{\mathrm{SiN}} \mathrm{S}$ multilayers.

\begin{tabular}{|c|c|c|c|c|c|c|c|c|c|}
\hline \multirow{2}{*}{ Multi-Layers } & \multicolumn{3}{|c|}{ MeN $(\mathrm{Me}=\mathrm{Zr}, \mathrm{Cr}, \mathrm{Al})$ Sublayer } & \multicolumn{3}{|c|}{$\mathrm{SiN}_{\mathrm{x}}$ Sublayer } & \multirow{2}{*}{$f_{\mathrm{MeN}}$} & \multirow{2}{*}{$\begin{array}{c}\text { Number of } \\
\text { Bilayers }\end{array}$} & \multirow{2}{*}{$\begin{array}{c}\text { Total } \\
\text { Thickness } \\
\text { (nm) }\end{array}$} \\
\hline & $\begin{array}{l}h_{\mathrm{MeN}} \\
(\mathrm{nm})\end{array}$ & $\begin{array}{c}\rho_{\mathrm{MeN}}(\mathrm{g} \\
\left.\mathrm{cm}^{-3}\right)\end{array}$ & $\begin{array}{c}w_{\mathrm{MeN}} \\
(\mathrm{nm})\end{array}$ & $\begin{array}{l}h_{\mathrm{SiNx}} \\
(\mathrm{nm})\end{array}$ & $\begin{array}{c}\rho_{\mathrm{SiNx}}(\mathrm{g} \\
\left.\mathrm{cm}^{-3}\right)\end{array}$ & $\begin{array}{c}w_{\mathrm{SiNx}} \\
(\mathrm{nm})\end{array}$ & & & \\
\hline \multicolumn{10}{|c|}{$\mathrm{ZrN} / \mathrm{SiN}_{\mathrm{x}}$} \\
\hline $5 \mathrm{~nm} / 5 \mathrm{~nm}$ & 3.7 & 7.3 & 0.4 & 5.3 & 3.1 & 0.6 & 0.41 & 29 & 261 \\
\hline $5 \mathrm{~nm} / 10 \mathrm{~nm}$ & 4.9 & 7.4 & 0.7 & 9.9 & 2.9 & 0.5 & 0.33 & 20 & 296 \\
\hline $2 \mathrm{~nm} / 5 \mathrm{~nm}$ & 1.6 & 7.4 & 0.6 & 5.0 & 3.0 & 0.6 & 0.24 & 43 & 284 \\
\hline \multicolumn{10}{|c|}{$\mathrm{CrN} / \mathrm{SiN}_{\mathrm{x}}$} \\
\hline $5 \mathrm{~nm} / 5 \mathrm{~nm}$ & 4.5 & 6.2 & 0.6 & 4.9 & 3.0 & 0.3 & 0.48 & 30 & 282 \\
\hline $5 \mathrm{~nm} / 10 \mathrm{~nm}$ & 4.4 & 6.4 & 0.4 & 9.7 & 3.0 & 0.3 & 0.31 & 20 & 282 \\
\hline $2 \mathrm{~nm} / 5 \mathrm{~nm}$ & 1.6 & 6.2 & 0.3 & 5.1 & 3.0 & 0.3 & 0.24 & 43 & 288 \\
\hline \multicolumn{10}{|c|}{$\operatorname{AlN} / \operatorname{SiN}_{x}$} \\
\hline $5 \mathrm{~nm} / 5 \mathrm{~nm}$ & 4.3 & 3.2 & 0.2 & 5.6 & 3.0 & 0.8 & 0.43 & 30 & 297 \\
\hline $5 \mathrm{~nm} / 10 \mathrm{~nm}$ & 3.8 & 3.3 & $<0.1$ & 11.3 & 3.0 & 0.4 & 0.25 & 20 & 302 \\
\hline $2 \mathrm{~nm} / 5 \mathrm{~nm}$ & 1.6 & 3.2 & 0.2 & 5.6 & 3.0 & 0.8 & 0.22 & 43 & 310 \\
\hline
\end{tabular}

The evolution of intrinsic stress developed during growth was monitored in situ and in real-time using the wafer curvature technique [33]. A multiple-beam optical stress sensor (MOSS) designed by kSpace Associates (kSA, Dexter, MI, USA) was implemented in the deposition chamber. The measurements were performed using $150 \pm 2 \mu \mathrm{m}$ thick Si substrate covered with native oxide, under stationary mode.

High resolution transmission electron microscopy (HRTEM, JEOL JEM ARM200F, Tokyo, Japan) analysis was carried out for direct information about the film structure and state of interlayer boundaries. Cross-sectional TEM specimens were prepared using a FEI Helios Nanolab 650 focused ion beam (FIB) (Brno, Czech Republic). More details on the sample preparation can be found in [6]. All specimens 
were analyzed using a JEOL JEM $2100 \mathrm{LaB}_{6}$ transmission electron microscope (Tokyo, Japan) operating at $200 \mathrm{kV}$.

The characterization of the multilayer stacking was carried out using low-angle X-ray Reflectivity (XRR). A fitting procedure, based on the optical formalism of Parratt [34], was used to extract the relevant quantities (individual layer thickness, mass density and interface roughness). The fraction of $\mathrm{MeN}(\mathrm{Me}=\mathrm{Zr}, \mathrm{Cr}, \mathrm{Al})$ layer is defined as $f_{\mathrm{MeN}}=\frac{h_{\mathrm{MeN}}}{h_{\mathrm{MeN}}+h_{\mathrm{SiNx}}}$, where $h_{\mathrm{MeN}}$ and $h_{\mathrm{SiN}_{\mathrm{x}}}$ are the MeN and $\mathrm{SiN}_{\mathrm{x}}$ layer thicknesses, respectively.

The elemental composition of films in their as-deposited (for the reference films only) and air-annealed states was determined using elemental probe microanalysis. A wavelength dispersive spectrometer (WDS) unit from Oxford Instruments (High Wycombe, UK) attached to a JEOL 7001 TTLS scanning electron microscope (Tokyo, Japan) operated at $10 \mathrm{kV}$ and $10 \mathrm{nA}$ was used for the quantification with a precision better than 1 at.\%. The same microscope was applied for obtaining top-view SEM micrographs of the films after air annealing at $950{ }^{\circ} \mathrm{C}$.

X-ray Diffraction (XRD) analysis was employed for structural identification using a Bruker D8 AXS $X$-ray diffractometer (Karlsruhe, Germany) operating in Bragg-Brentano configuration and equipped with $\mathrm{Cu}_{\mathrm{K} \alpha}$ wavelength $(0.15418 \mathrm{~nm})$ and LynxEye detector.

Isothermal air annealing was performed at different sequential temperatures from 400 to $950{ }^{\circ} \mathrm{C}$. The oxidation process was investigated using in situ XRD experiments. The samples were placed on a resistive heating stage designed by Anton Paar (Graz, Austria) implemented on the Bruker D8 diffractometer, consisting in an AIN sample holder and a hemispheric graphite dome subjected to air blowing. Total scan time during isothermal annealing was 50-60 min.

\section{Results and Discussion}

\subsection{Structure and Phase Composition of As-Deposited MeN/SiN $\mathrm{N}_{x}(\mathrm{Me}=\mathrm{Zr}, \mathrm{Cr}, \mathrm{Al})$ Multilayered Films}

As described in Section 2, the $\mathrm{ZrN} / \mathrm{SiN}_{x}, \mathrm{CrN} / \mathrm{SiN}_{\mathrm{x}}$ and $\mathrm{AlN} / \mathrm{SiN}_{\mathrm{x}}$ multilayered films with different thickness ratio of $\mathrm{MeN}(\mathrm{Me}=\mathrm{Zr}, \mathrm{Cr}, \mathrm{Al})$ and $\mathrm{SiN}_{\mathrm{x}}$ elementary layers were formed. This corresponds to different $\mathrm{MeN}$ fractions, $f_{\mathrm{MeN}}$. The elemental composition of the reference monolithic films, obtained from WDS, is given in Table $1 . \mathrm{MeN}_{\mathrm{x}}$ films are slightly off-stoichiometric: overstoichiometric $\mathrm{ZrN}_{1.15}$ and $\mathrm{AlN}_{1.30}$ films and substoichiometric $\mathrm{CrN}_{0.83}$ films were obtained. However, the $\mathrm{SiN}_{\mathrm{x}}$ film has the $\mathrm{Si}_{3} \mathrm{~N}_{4}$ stoichiometry.

Figure 1 shows the HRTEM images of $\mathrm{MeN} / \mathrm{SiN}_{\mathrm{x}}(\mathrm{Me}=\mathrm{Zr}, \mathrm{Cr}, \mathrm{Al})$ multilayered films with the ratio of elementary layer thicknesses equal to $5 \mathrm{~nm} / 5 \mathrm{~nm}$. It reveals the uniform alternation of MeN and $\mathrm{SiN}_{\mathrm{x}}$ elementary layers, and the formation of sharp and smooth interfaces between the layers points to the absence of intermixing of the layer components. However, the interlayer boundary seems more diffuse for the $\mathrm{CrN} / \mathrm{SiN}_{\mathrm{x}}$ multilayer, possibly due to the growth at higher substrate temperature. While the $\mathrm{MeN}_{\mathrm{x}}$ layers appear crystalline (see, e.g., lattice fringes in AlN layer of Figure 1c), the SiN layers were found to be amorphous. Note also a change in electronic contrast from $\mathrm{ZrN} / \mathrm{SiN}_{\mathrm{x}}$ to $\mathrm{AlN} / \mathrm{SiN}_{\mathrm{x}}$ multilayers, due to reduction in mass density difference between $\mathrm{MeN}$ and $\mathrm{SiN}_{\mathrm{x}}$ layers. The values of mass densities for the elementary layers are presented in Table 2, as extracted from the fit of XRR data (see below). In particular, the contrast between $\mathrm{AlN}$ and $\mathrm{SiN}_{\mathrm{X}}$ layers is very similar, as also observed by other authors [27].

Figure 2 shows the low-angle XRR scans together with the simulated curves obtained for MeN/SiN $(\mathrm{Me}=\mathrm{Zr}, \mathrm{Cr}, \mathrm{Al})$ multilayered films with the nominal MeN thickness $h_{\mathrm{MeN}}=5 \mathrm{~nm}$ and $\mathrm{SiN}_{\mathrm{x}}$ thickness $h_{\mathrm{SiNx}}=5 \mathrm{~nm}$. For all multilayered systems, relatively sharp superlattice reflections are observed up to high $2 \theta$ angles (up to seventh order or more). This testifies the formation of highly periodic multilayer structures and gives evidence of the presence of relatively smooth interface boundaries between the layers. Similar results were obtained for $\mathrm{CrN} / \mathrm{Si}_{3} \mathrm{~N}_{4}$ multilayered films in [20], where the effectiveness of $\mathrm{Si}_{3} \mathrm{~N}_{4}$ layer for stabilization of periodic structure was mentioned. Note that Kiessig fringes are also 
distinguishable between main superlattice reflections in XRR scans (however, not visible in the scale displayed in Figure 2).

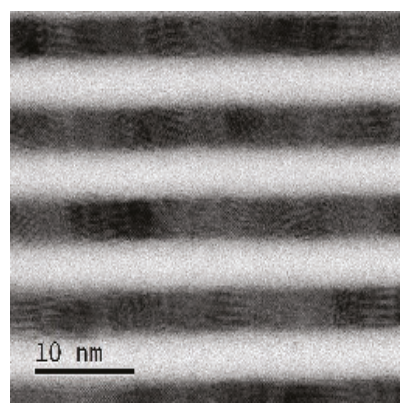

(a)

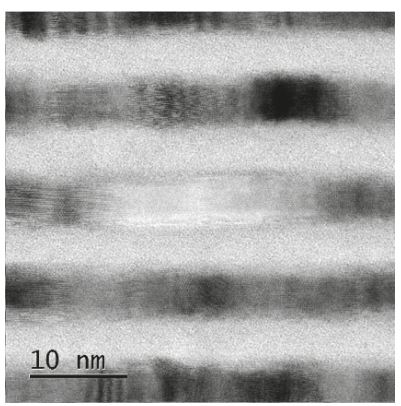

(b)

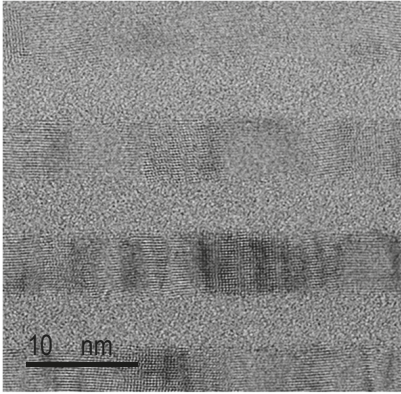

(c)

Figure 1. Cross-sectional (bright field) HRTEM images of multilayered films with the ratio of thicknesses of $\mathrm{MeN}$ (darker contrast) and $\mathrm{SiN}_{\mathrm{x}}$ (brighter contrast) elementary layers equal to $5 \mathrm{~nm} / 5 \mathrm{~nm}: \mathrm{ZrN}_{\mathrm{riN}}$ (a); $\mathrm{CrN} / \mathrm{SiN}_{\mathrm{x}}(\mathbf{b})$; and $\mathrm{AlN} / \mathrm{SiN}_{\mathrm{x}}$ (c).

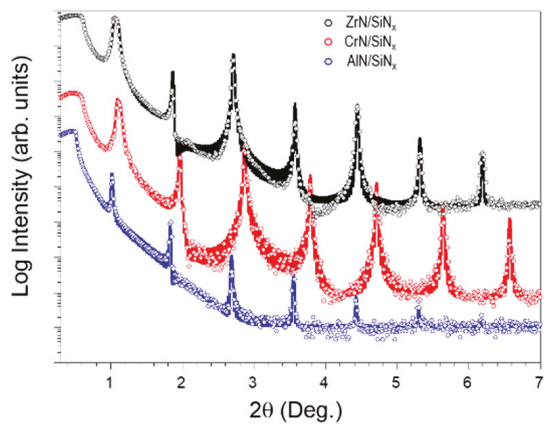

Figure 2. $\mathrm{XRR}$ scans of $\mathrm{ZrN} / \mathrm{SiN}_{\mathrm{x}}, \mathrm{CrN} / \mathrm{SiN}_{\mathrm{x}}$ and $\mathrm{AIN} / \mathrm{SiN}_{\mathrm{x}}$ multilayered films with the ratio of thicknesses of MeN and $\mathrm{SiN}_{\mathrm{x}}$ elementary layers equal to $5 \mathrm{~nm} / 5 \mathrm{~nm}$. Solid lines correspond to the best-fit simulations to experimental data using optical model of Parratt.

An optical simulation model was used to fit XRR data and get information on layer density, layer thickness and interface roughness of each sublayer for $\mathrm{ZrN} / \mathrm{SiN}, \mathrm{CrN} / \mathrm{SiN}_{\mathrm{x}}$ and $\mathrm{AlN} / \mathrm{SiN}_{\mathrm{x}}$ multilayers. Values are reported in Table 2 for the different systems and bilayer periods investigated. The obtained results show that the boundaries between the layers for all multilayered systems are quite smooth (the interface roughness $w_{\mathrm{MeN}}$ does not exceed $0.6 \mathrm{~nm}$ ), which is in good agreement with the HRTEM observations presented in Figure 1. All $\mathrm{MeN}_{\mathrm{x}}$ sublayers were found to be dense, with mass density of $\rho_{\mathrm{ZrN}}=7.4 \pm 0.1 \mathrm{~g} \cdot \mathrm{cm}^{-3}, \rho_{\mathrm{CrN}}=6.3 \pm 0.1 \mathrm{~g} \cdot \mathrm{cm}^{-3}$ and $\rho_{\mathrm{AlN}}=3.2 \pm 0.1 \mathrm{~g} \cdot \mathrm{cm}^{-3}$. These values are very close to the values for bulk reference powders, being $\rho_{\mathrm{ZrN}}=7.29 \mathrm{~g} \cdot \mathrm{cm}^{-3}, \rho_{\mathrm{CrN}}=6.18 \mathrm{~g} \cdot \mathrm{cm}^{-3}$ and $\rho_{\text {AlN }}=3.26 \mathrm{~g} \cdot \mathrm{cm}^{-3}$. The value $\rho_{\mathrm{SiNx}}$ was found to be equal to $3.0 \mathrm{~g} \cdot \pm 0.1 \mathrm{~cm}^{-3}$, also in good agreement with the bulk value of $\alpha-\mathrm{Si}_{3} \mathrm{~N}_{4}$ crystalline phase $\left(3.18 \mathrm{~g} \cdot \mathrm{cm}^{-3}\right)$.

One can note from the results in Table 2 that for all multilayered films the thickness of $\mathrm{MeN}$ $(\mathrm{Me}=\mathrm{Zr}, \mathrm{Cr}, \mathrm{Al})$ layer is less than the nominal thickness. For example, for the case of nominal ratio of $\mathrm{MeN}$ and $\mathrm{SiN}_{\mathrm{x}}$ thicknesses $2 \mathrm{~nm} / 5 \mathrm{~nm}$, the real thickness of MeN layer is $1.6 \mathrm{~nm}$, i.e. $20 \%$ less. This is related to the poisoning of the metal target during the first seconds of the reactive sputter-deposition process. Consequently, the initial deposition rate is lower than the average value calculated from the 
thicker reference MeN films. More information on this poisoning effect can be found in [23]. At the same time, the thickness of $\mathrm{SiN}_{\mathrm{x}}$ layer is close to nominal one for all multilayered systems (Table 2).

Figure 3 shows the substrate curvature change measured by MOSS during growth of $\mathrm{ZrN} / \mathrm{SiN}_{\mathrm{x}}$, $\mathrm{CrN} / \mathrm{SiN}_{\mathrm{X}}$ and $\mathrm{AlN} / \mathrm{SiN}_{\mathrm{X}}$ multilayered films with nominal thickness ratio $10 \mathrm{~nm} / 5 \mathrm{~nm}$. One can see from the slope of F/w vs. layer thickness that both sublayers develop compressive stress. However, the magnitude of the compressive stress is much larger for $\mathrm{MeN}$ layers than for $\mathrm{SiN}_{\mathrm{x}}$ layers. Relatively large values of -7.2 and $-5.7 \mathrm{GPa}$ were obtained for the incremental stress during deposition of AlN and $\mathrm{ZrN}$ layers, respectively, while for $\mathrm{CrN}$ layers the incremental stress is $-2.7 \mathrm{GPa}$. Comparatively, the incremental stress of $\mathrm{SiN}_{\mathrm{x}}$ layer is about $-1.2 \mathrm{GPa}$. One can also notice from the results in Figure 3 that the stress state is reproducible from one bilayer to another, i.e., there is no influence of the underneath layers on the cumulative stress build-up. The formation of compressive stress in sputter-deposited TMN layers is due to energetic bombardment during growth, which creates point defects in the crystal lattice and densify the grain boundaries [23,35-37]. At low deposited energy, sputter-deposited $\mathrm{MeN}_{\mathrm{x}}$ films develop a columnar, underdense microstructure, often accompanied by the development of tensile stress $[35,37,38]$. This is obviously not the case here for these nanoscale layer thicknesses, because the presence of amorphous $\mathrm{SiN}_{\mathrm{x}}$ layers interrupts the columnar growth of MeN layers. If the energy delivered to growing film is large, a dense and sometimes featureless microstructure is formed together with compressive stress. In our deposition conditions, the main contribution to the deposited energy stems from energetic neutrals (sputtered atoms and backscattered Ar) due to low fraction of bombarding ions (a few percent) reaching the substrate. The lower compressive stress values for $\mathrm{CrN}$ layers is likely due to the higher substrate temperature $\left(450{ }^{\circ} \mathrm{C}\right.$ vs. $\left.300^{\circ} \mathrm{C}\right)$, which favors defect annihilation processes. Amorphous layers are more tolerant to defect incorporation compared to their crystalline counterpart, which explains the lower stress values obtained for $\mathrm{SiN}_{\mathrm{x}}$.
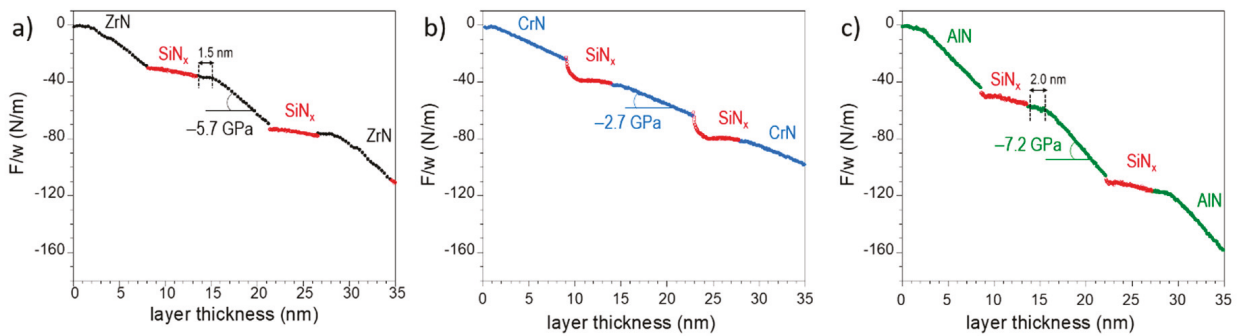

Figure 3. In situ stress evolution during growth of multilayered films: $\mathrm{ZrN}_{\mathrm{SiN}}(\mathbf{a}) ; \mathrm{CrN} / \mathrm{SiN}_{\mathrm{x}}(\mathbf{b})$; and $\mathrm{AIN} / \mathrm{SiN}_{\mathrm{x}}$ (c). The data were obtained from MOSS and are displayed as the variation of the force per unit width $(\mathrm{F} / \mathrm{w})$ vs. layer thickness. The slope of the curves gives the information on the incremental stress, ranging from $-7.2 \mathrm{GPa}(\mathrm{AlN})$ to $-2.7 \mathrm{GPa}(\mathrm{CrN})$.

A closer inspection to the stress curves of Figure 3 reveals some interesting features. The development of compressive stress starts after $\sim 1.5 \mathrm{~nm}$ and $2.0 \mathrm{~nm}$ for $\mathrm{ZrN}$ and AlN layers, respectively. Below these values, the stress is relatively small (about $-1 \mathrm{GPa}$ ) and could reflect the initial formation of an amorphous layer. XRD characterizations on the MeN/SiN $\mathrm{x}_{\mathrm{x}}$ multilayers with $2 \mathrm{~nm} / 5 \mathrm{~nm}$ thickness ratio support this scenario. Finally, it can be noticed in Figure $3 b$ that the stress development during growth of $\mathrm{SiN}_{x}$ on $\mathrm{CrN}$ is peculiar, as a steady-state stress is only reached after $\sim 2-3 \mathrm{~nm}$. The larger compressive stress that develops in the very beginning is contributed to interface stress and possible interlayer formation, as also noticed from cross-section HRTEM view in Figure 1b.

In Figures 4-6 (see black lines for as-deposited state), the XRD patterns of reference monolithic $\mathrm{ZrN}$ (Figure 4a), $\mathrm{CrN}$ (Figure 5a) and AIN (Figure 6a) films are represented in comparison with the XRD patterns of $\mathrm{MeN} / \mathrm{SiN}_{\mathrm{X}}(\mathrm{Me}=\mathrm{Zr}, \mathrm{Cr}, \mathrm{Al}$ ) multilayered films (Figure $4 \mathrm{~b}-\mathrm{d}$, Figures $5 \mathrm{~b}-\mathrm{d}$ and $6 \mathrm{~b}-\mathrm{d}$ ). The angular range $25-58^{\circ}$ covers the main 111 and 200 Bragg reflections for cubic c-ZrN (JCPDS card 
No. 35-0753), c-CrN (JCPDS card No. 76-2494) as well as 100, 002, 101 and 102 reflections for hexagonal h-AlN with wurtzite structure (JCPDS card No. 25-1133).

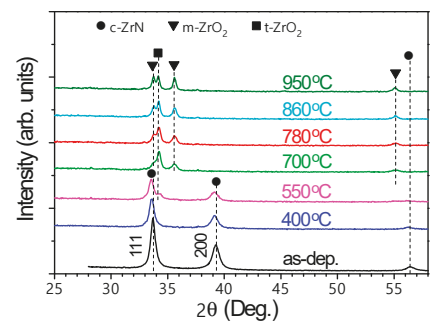

(a)

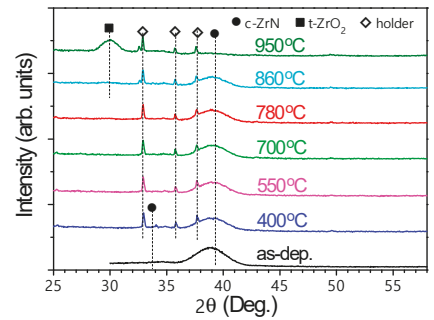

(c)

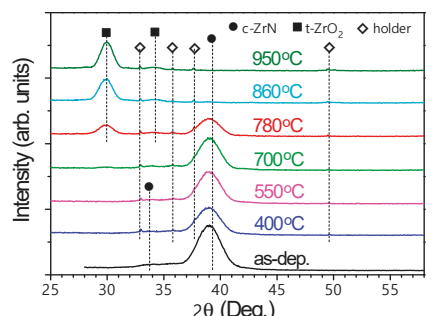

(b)

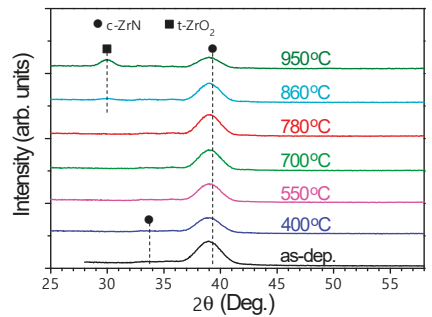

(d)

Figure 4. Evolution of XRD patterns under air annealing for $\mathrm{ZrN}$ reference film (a); and $\mathrm{ZrN} / \mathrm{SiN}_{\mathrm{x}}$ multilayered films with different thickness of $\mathrm{ZrN}_{\text {and }} \mathrm{SiN}_{\mathrm{x}}$ elementary layers: (b) $5 \mathrm{~nm} / 2 \mathrm{~nm}$; (c) 5 $\mathrm{nm} / 5 \mathrm{~nm}$; and (d) $5 \mathrm{~nm} / 10 \mathrm{~nm}$.

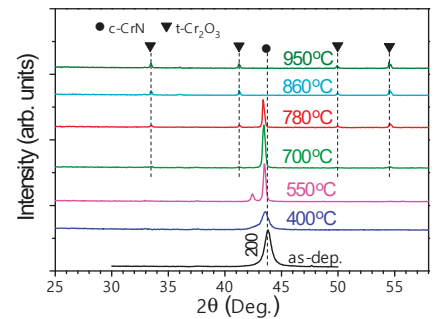

(a)

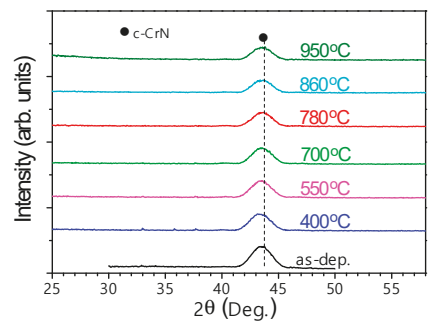

(c)

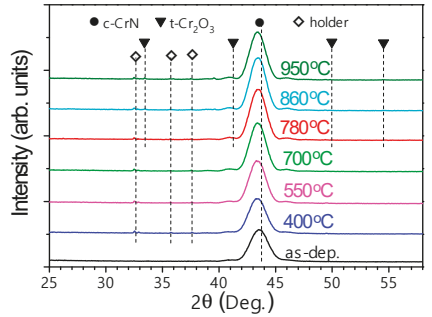

(b)

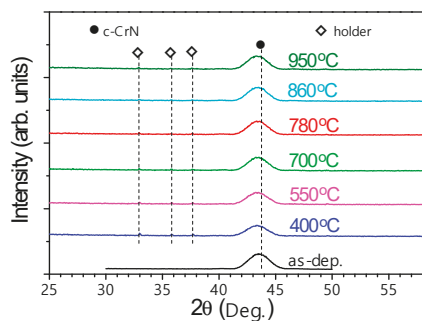

(d)

Figure 5. Evolution of $\mathrm{XRD}$ patterns under air annealing for $\mathrm{CrN}$ reference film (a); and $\mathrm{CrN} / \mathrm{SiN}_{\mathrm{x}}$ multilayered films with different thickness of $\mathrm{CrN}$ and $\mathrm{SiN}_{\mathrm{X}}$ elementary layers: (b) $5 \mathrm{~nm} / 2 \mathrm{~nm}$; (c) 5 $\mathrm{nm} / 5 \mathrm{~nm}$; and (d) $5 \mathrm{~nm} / 10 \mathrm{~nm}$. 


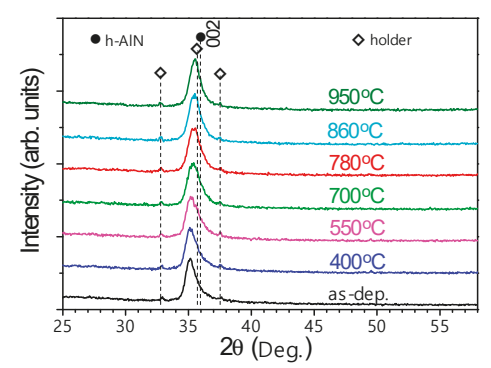

(a)

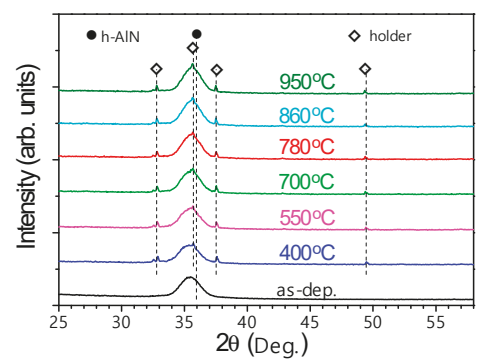

(c)

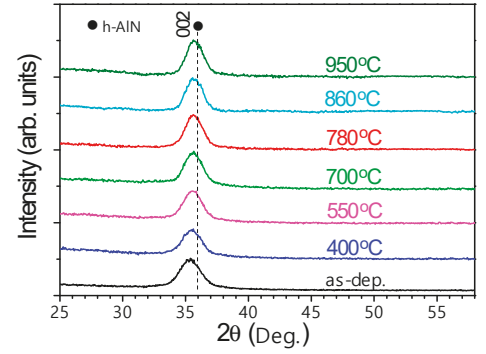

(b)

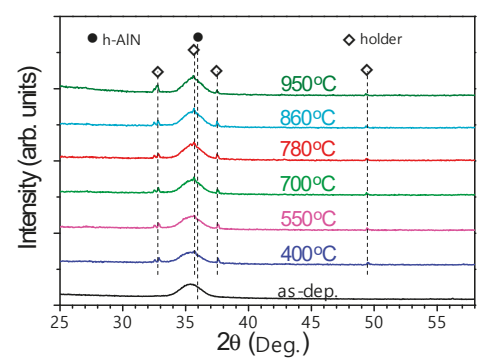

(d)

Figure 6. Evolution of XRD patterns under air annealing for AlN reference film (a); and $\mathrm{AlN} / \mathrm{SiN}_{\mathrm{x}}$ multilayered films with different thickness of AlN and $\mathrm{SiN}_{x}$ elementary layers: (b) $5 \mathrm{~nm} / 2 \mathrm{~nm}$; (c) 5 $\mathrm{nm} / 5 \mathrm{~nm}$; and (d) $5 \mathrm{~nm} / 10 \mathrm{~nm}$.

If for the reference c-ZrN film (Figure 4a) both (111) and (200) preferred orientations take place, in the case of reference c-CrN and h-AlN films, the preferred orientation along the [200] direction (c-CrN) or in the [002] direction (h-AlN) is only observed (Figures 5a and 6a). Competitive columnar growth between (111)- and (200)-oriented $\mathrm{ZrN}$ crystallites during sputter-deposition of $\mathrm{ZrN}$ monolithic films was discussed previously [23,35]. The (200) preferred orientation is characteristic for $\mathrm{CrN}$ films [39,40], as well as (002) preferred orientation is typical for h-AlN films [41-43]. Torino et al. [42] reported a transition from (101) to (002) preferred orientation for AlN films with decreasing $\left(\mathrm{Ar}+\mathrm{N}_{2}\right)$ working pressure in the chamber. The presence of (002) orientation means that the AlN crystallites are highly oriented with the c-axis perpendicular to the substrate surface [43,44].

It was observed that the peak position of reference $\mathrm{ZrN}, \mathrm{CrN}$ and $\mathrm{AlN}$ monolithic films is shifted to lower $2 \theta$ angles as compared to the position for bulk materials. This is related to the presence of in-plane compressive stresses, resulting in an expansion of the out-of-plane lattice parameter. For the AlN reference film, an asymmetry of the 002 peak towards higher $2 \theta$ angles was noticed, which can be due to a higher defect concentration. The reference $\mathrm{Si}_{3} \mathrm{~N}_{4}$ film was found to be $\mathrm{X}$-ray amorphous, i.e., there are no reflections in the investigated angular range (see [23]).

If we now analyze the XRD patterns of $\mathrm{MeN} / \mathrm{SiN}_{\mathrm{x}}(\mathrm{Me}=\mathrm{Zr}, \mathrm{Cr}, \mathrm{Al})$ multilayers in their as-deposited state, the following observations can be made in comparison with the reference MeN films: (i) a transition from (111) preferred orientation, which predominates for reference $\mathrm{ZrN}$ film (Figure 4a), to (200) orientation for $\mathrm{ZrN} / \mathrm{SiN}_{\mathrm{x}}$ multilayered films (Figure $4 \mathrm{~b}-\mathrm{d}$ ) and the retaining of (200) or (002) preferred orientation for $\mathrm{CrN} / \mathrm{SiN}_{\mathrm{x}}$ (Figure $5 \mathrm{~b}-\mathrm{d}$ ) or $\mathrm{AlN} / \mathrm{SiN}_{\mathrm{x}}$ (Figure $6 \mathrm{~b}-\mathrm{d}$ ) multilayered films as in the case of corresponding mononitrides CrN (Figure 5a) or AlN (Figure 6a); (ii) the broadening of the MeN XRD lines and decrease in their intensity that can be caused by the decrease in MeN crystallites size; and (iii) the amorphization of the $\mathrm{MeN}_{\mathrm{SiN}}(\mathrm{Me}=\mathrm{Zr}, \mathrm{Cr}, \mathrm{Al})$ multilayered films when the $\mathrm{MeN}$ 
layer thickness decreases down to $2 \mathrm{~nm}$ (XRD patterns of the $\mathrm{ZrN} / \mathrm{SiN}_{\mathrm{x}}$ multilayer with $2 \mathrm{~nm} / 5 \mathrm{~nm}$ thickness ratio can be found in our previous works $[6,23])$.

In the case of $\mathrm{ZrN} / \mathrm{SiN}_{\mathrm{x}}$ multilayered films, the amorphous $\mathrm{SiN}_{\mathrm{x}}$ layer hinders the columnar growth of $\mathrm{ZrN}$ crystallites and favors the (200) preferred orientation [23]. The transition to (200) preferred orientation and broadening of $\mathrm{ZrN}$ peak with decreasing $f_{\mathrm{MeN}}$ was also observed by Dong et al. [25]. $\mathrm{CrN} / \mathrm{SiN}_{\mathrm{x}}$ multilayered films were also characterized by (200) preferred orientation [20]. The present results allow concluding that the insertion of amorphous $\mathrm{SiN}_{\mathrm{x}}$ layers influence the structure of growing MeN layers.

As for the reference $\mathrm{MeN}_{\mathrm{x}}$ films, the position of $\mathrm{ZrN}$ and $\mathrm{CrN} 200$ lines, and AlN 002 line is shifted to lower angles compared to position for bulk materials, in good agreement with an in-plane compressive stress state, as revealed from substrate curvature measurements (see Figure 3).

\subsection{Evolution of Phase Composition of $\mathrm{MeN} / \mathrm{SiN}_{x}(\mathrm{Me}=\mathrm{Zr}, \mathrm{Cr}, \mathrm{Al})$ Multilayered Films during Air Annealing}

The evolution of XRD patterns for reference (monolithic) single-layer MeN and for multilayered films of different composition with the increase of air-annealing temperature from of 400 to $950{ }^{\circ} \mathrm{C}$ is shown in Figures 4-6. When considering the reference $\mathrm{ZrN}$ (Figure 4a), $\mathrm{CrN}$ (Figure $5 \mathrm{a}$ ) and AlN (Figure 6a) films, it is worth noting the following. Oxidation of $\mathrm{ZrN}$ starts already at the temperature of $550{ }^{\circ} \mathrm{C}\left(\mathrm{t}-\mathrm{ZrO}_{2}\right.$ phase is registered), oxidation of $\mathrm{CrN}$ at the temperature of $700{ }^{\circ} \mathrm{C}$ (diffraction reflections of $\mathrm{t}-\mathrm{Cr}_{2} \mathrm{O}_{3}$ phase appear), while the AlN film remains unaltered up to $950{ }^{\circ} \mathrm{C}$. This shows the higher thermal stability of AlN. The peaks of $\mathrm{ZrN}$ and $\mathrm{CrN}$ phases disappear completely when reaching the temperature of 700 and $860^{\circ} \mathrm{C}$, respectively. It should be noted that the shoulder to the left from $200 \mathrm{c}-\mathrm{CrN}$ peak is detected for $\mathrm{CrN}$ film at the temperature of $400{ }^{\circ} \mathrm{C}$, and afterwards the weak 111 reflection of $\mathrm{h}-\mathrm{Cr}_{2} \mathrm{~N}$ phase (JCPDS card No. 35-0803) at $2 \theta \approx 42.6^{\circ}$ is registered at $550^{\circ} \mathrm{C}$ (Figure 5a). Previous studies have also reported the transformation from c-CrN to h- $\mathrm{Cr}_{2} \mathrm{~N}$ phase during vacuum or air annealing $[45,46]$ due to depletion in nitrogen. Both phases decompose with the formation of chromium oxides at the subsequent temperature rise.

In the case of $\mathrm{ZrN} / \mathrm{SiN}_{\mathrm{x}}$ multilayered films (Figure $4 \mathrm{~b}-\mathrm{d}$ ), the evolution of XRD patterns during air annealing depends on the $f_{\mathrm{MeN}}$ fraction. For $\mathrm{ZrN}_{\mathrm{SiN}}(5 \mathrm{~nm} / 2 \mathrm{~nm})$, for which $f_{\mathrm{MeN}}>0.50$, the crystallization of $\mathrm{t}-\mathrm{ZrO}_{2}$ oxide phase occurs at the temperatures of $700-780{ }^{\circ} \mathrm{C}$ (Figure $4 \mathrm{~b}$ ). When reaching the temperature of $860{ }^{\circ} \mathrm{C}$, the $200 \mathrm{ZrN}$ peak disappears, indicating the decomposition of the nitride phase and formation of zirconium oxides. For $\mathrm{ZrN}_{\mathrm{SiN}}(5 \mathrm{~nm} / 5 \mathrm{~nm})$ and $\mathrm{ZrN} / \mathrm{SiN}_{\mathrm{x}}(5 \mathrm{~nm} / 10$ $\mathrm{nm}$ ) films, for which $f_{\mathrm{MeN}}<0.50$ (Table 2), the crystallization of $\mathrm{t}-\mathrm{ZrO}_{2}$ takes place at the higher temperatures $\left(860-950{ }^{\circ} \mathrm{C}\right)$. In the case of $\mathrm{ZrN}_{\mathrm{SiN}}(5 \mathrm{~nm} / 10 \mathrm{~nm})$, the $\mathrm{ZrN}$ peak remains until $950{ }^{\circ} \mathrm{C}$ (Figure 4d). Therefore, it can be concluded that oxidation resistance of $\mathrm{ZrN} / \mathrm{SiN}_{\mathrm{x}}$ multilayers increases with increasing $\mathrm{SiN}_{\mathrm{x}}$ layer thickness from 2 to $10 \mathrm{~nm}$ at the constant thickness of $5 \mathrm{~nm}$ for $\mathrm{ZrN}$ layer. A similar trend of thermal stability enhancement with decreasing $f_{\mathrm{MeN}}$ fraction was reported earlier [6]. Note that the $\mathrm{ZrN} / \mathrm{SiN}_{\mathrm{x}}(2 \mathrm{~nm} / 5 \mathrm{~nm})$ amorphous multilayer film was also stable in the temperature range of $400-950{ }^{\circ} \mathrm{C}$, with no oxide phases detected (see Figure 13d in [6]). The value of $f_{\mathrm{MeN}}$ for this multilayer is similar to that for $\mathrm{ZrN}_{\mathrm{SiN}}(5 \mathrm{~nm} / 10 \mathrm{~nm})$ multilayer but the interface density is more than double (Table 2). This suggests that an increase in interface density also promotes the oxidation resistance of $\mathrm{ZrN} / \mathrm{SiN}_{\mathrm{x}}$ multilayered films.

Contrarily to the reference $\mathrm{CrN}$ film (Figure $5 \mathrm{a}$ ), $\mathrm{CrN} / \mathrm{SiN}_{\mathrm{x}}$ multilayers were found to be thermally stable up to $950{ }^{\circ} \mathrm{C}$. No crystalline oxide phases are detected up to temperature of $950{ }^{\circ} \mathrm{C}$. For the $\mathrm{CrN} / \mathrm{SiN}_{\mathrm{x}}(5 \mathrm{~nm} / 2 \mathrm{~nm}$ ) multilayered films (Figure $5 \mathrm{~b}$ ), the intensity of $200 \mathrm{CrN}$ peak even slightly increases with temperature, which is most likely connected to some improvement of crystalline quality of this film. The $\mathrm{CrN} / \mathrm{SiN}_{\mathrm{x}}(2 \mathrm{~nm} / 5 \mathrm{~nm})$ film, which was amorphous in its as-deposited state, remains amorphous after air annealing (not shown).

For the third multilayered system, i.e. for $\mathrm{AlN} / \mathrm{SiN}_{\mathrm{x}}$ films, the emergence of oxide phases during air annealing is not registered for all studied thickness ratios of elementary layers, namely, $5 \mathrm{~nm} / 2 \mathrm{~nm}$, $5 \mathrm{~nm} / 5 \mathrm{~nm}, 5 \mathrm{~nm} / 10 \mathrm{~nm}$ and $2 \mathrm{~nm} / 5 \mathrm{~nm}$ (Figure 6b-d). Intensity of 002 AlN peak rises slightly with the 
increase in annealing temperature for all samples except for $\mathrm{AlN}_{\mathrm{SiN}}(2 \mathrm{~nm} / 5 \mathrm{~nm})$ film, which remains X-ray amorphous.

To get more insights on phase stability, we plot in Figure 7 the evolution of the out-of-plane lattice parameter of MeN layers with annealing temperature. Lattice parameter $a$ of c- $\mathrm{ZrN}$ and c-CrN phases was calculated using angular position of 200 peak, and lattice parameter $c$ of h-AlN phase using 002 peak. The results are shown for the reference $\mathrm{ZrN}, \mathrm{CrN}$ and $\mathrm{AlN}$ films, as well as for MeN/SiN $(\mathrm{Me}=\mathrm{Zr}, \mathrm{Cr}, \mathrm{Al})$ multilayers with $5 \mathrm{~nm} / 5 \mathrm{~nm}$ and $5 \mathrm{~nm} / 10 \mathrm{~nm}$ ratios. For AlN sub-layers, as well as AlN monolithic film, a substantial decrease of the lattice parameter was found, up to $0.7 \%$ relative reduction for the reference AlN film. This is contributed to the relaxation of compressive stress with increasing annealing temperature, which decreases propensity of film delamination and/or bucking and is therefore beneficial to its thermal stability. An opposite behavior was found for $\mathrm{ZrN}$ and $\mathrm{CrN}$ reference films, which could only be explained by decomposition of the MeN phase due to nitrogen release. For $\mathrm{ZrN} / \mathrm{SiN}_{\mathrm{x}}$ and $\mathrm{CrN} / \mathrm{SiN}_{\mathrm{x}}$ multilayers, one observes a competition between nitrogen loss and relaxation of compressive stress, depending on the annealing temperature.

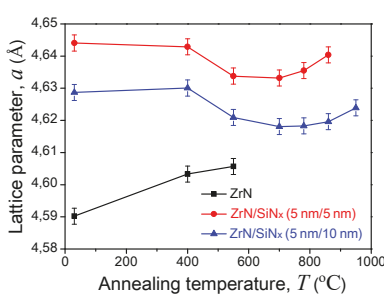

(a)

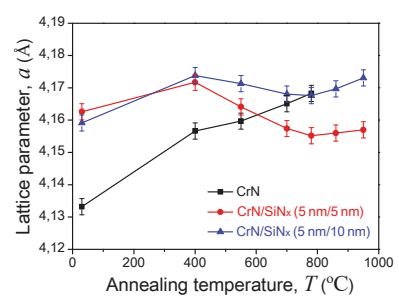

(b)

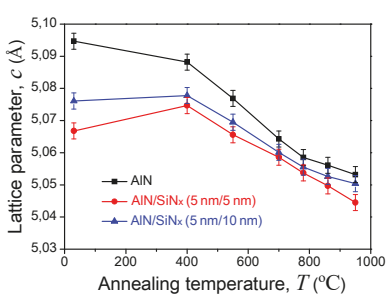

(c)

Figure 7. Dependences of the lattice parameter of MeN phase on the annealing temperature for multilayered films with comparison with the same dependences for reference monolithic films: $\mathrm{ZrN} / \mathrm{SiN}_{\mathrm{x}}(\mathbf{a}) ; \mathrm{CrN} / \mathrm{SiN}_{\mathrm{x}}(\mathbf{b}) ;$ and $\mathrm{AlN} / \mathrm{SiN}_{\mathrm{x}}$ (c).

\subsection{Elemental Composition and Surface State of $\mathrm{MeN} / \mathrm{SiN}_{x}(\mathrm{Me}=\mathrm{Zr}, \mathrm{Cr}, \mathrm{Al})$ Multilayered Films after Air} Annealing Procedure

The composition of the reference $\mathrm{ZrN}, \mathrm{CrN}, \mathrm{AlN}$ and $\mathrm{Si}_{3} \mathrm{~N}_{4}$ films as well as $\mathrm{MeN} / \mathrm{SiN}_{\mathrm{x}}(\mathrm{Me}=\mathrm{Zr}$, $\mathrm{Cr}, \mathrm{Al})$ multilayered films after air annealing up to $950{ }^{\circ} \mathrm{C}$ was measured by WDS. Concerning the multilayered films, it should be noted that by means of WDS the integral content of the elements (corresponding to the whole thickness of the coating) was determined. Consequently, the obtained data allowed carrying out the relative comparison of oxidation resistance of the multilayered films of three systems- $\mathrm{ZrN} / \mathrm{SiN}_{\mathrm{x}}, \mathrm{CrN} / \mathrm{SiN}_{\mathrm{x}}$ and $\mathrm{AlN} / \mathrm{SiN}_{\mathrm{x}}$ - with the same ratios of elementary layer thicknesses. The oxygen and nitrogen content in the film composition gives information on film stability after air-annealing. As the oxygen incorporates and substitutes the nitrogen atoms in MeN lattice during high-temperature annealing, the increase in oxygen content in the coating composition is always accompanied by a decrease in nitrogen content. The variation in oxygen content for the reference and multilayered films is displayed in Figure 8.

If the $\mathrm{ZrN}$ and $\mathrm{CrN}$ films are fully oxidized (the oxygen content is close to composition of $\mathrm{ZrO}_{2}$ and $\mathrm{Cr}_{2} \mathrm{O}_{3}$ oxides, respectively), the $\mathrm{AlN}$ and $\mathrm{Si}_{3} \mathrm{~N}_{4}$ films are characterized by a considerably lower degree of oxidation (around 30 at.\%, see Figure 8). Overall, the $\mathrm{MeN} / \mathrm{SiN}_{\mathrm{x}}$ multilayers show better oxidation resistance than their mononitride counterparts, in agreement with XRD analysis. For the $\mathrm{ZrN} / \mathrm{SiN}_{\mathrm{x}}$ system, the tendency of oxidation resistance enhancement with decreasing $f_{\text {MeN }}$ was clearly observed. In the case of $\mathrm{CrN} / \mathrm{SiN}_{\mathrm{x}}$ and $\mathrm{AlN} / \mathrm{SiN}_{\mathrm{x}}$ systems, the same tendency is revealed but it is much less pronounced. It is noteworthy that for $\mathrm{CrN} / \mathrm{SiN}_{\mathrm{x}}$ and $\mathrm{AlN} / \mathrm{SiN}_{\mathrm{x}}$ films the decrease in MeN layer thickness down to $2 \mathrm{~nm}$ leads to a certain deterioration of oxidation resistance. 


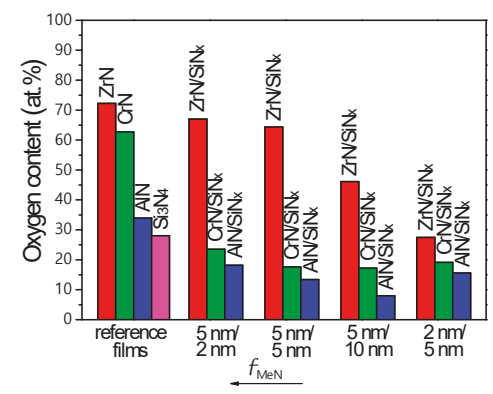

Figure 8. The oxygen content for the reference $\mathrm{ZrN}, \mathrm{CrN}, \mathrm{AlN}$, and $\mathrm{Si}_{3} \mathrm{~N}_{4}$ films as well as $\mathrm{ZrN} / \mathrm{SiN}_{\mathrm{x}}$, $\mathrm{CrN} / \mathrm{SiN}_{\mathrm{x}}$ and $\mathrm{AlN} / \mathrm{SiN}_{\mathrm{x}}$ multilayered films with different ratios of the thicknesses of $\mathrm{MeN}$ and $\mathrm{SiN}_{\mathrm{x}}$ elementary layers after annealing in air at $950^{\circ} \mathrm{C}$.

The results reported in Figure 8 allow concluding that $\mathrm{CrN} / \mathrm{SiN} \mathrm{x}_{\mathrm{x}}$ and $\mathrm{AlN} / \mathrm{SiN}_{\mathrm{x}}$ multilayered films are significantly more stable under conditions of high-temperature annealing as compared to $\mathrm{ZrN} / \mathrm{SiN}_{\mathrm{x}}$ films. These results agree with XRD data discussed in the previous section. The smallest content of oxygen (8 at. \%) after annealing in air was registered for $\operatorname{AlN} \mathrm{SiN}_{\mathrm{x}}(5 \mathrm{~nm} / 10 \mathrm{~nm})$ film.

To clarify the mechanisms responsible for film oxidation, the analysis of the surface state was performed by SEM after high-temperature $\left(950^{\circ} \mathrm{C}\right)$ annealing. SEM observations reveal that the high degree of the coating damage, namely the emergence of corrosion sites, the swelling and flacking of the film, is inherent to the reference monolithic films, except for AlN surface, which remains quite uniform after annealing (see Figure 9).

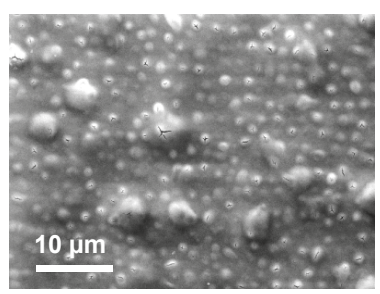

(a)

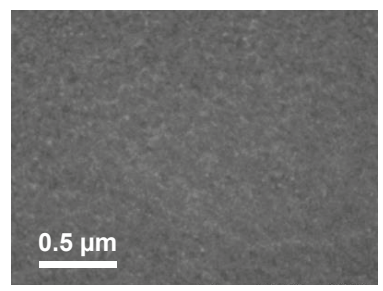

(c)

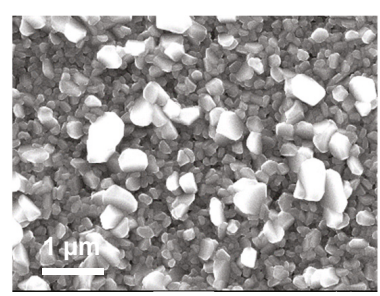

(b)

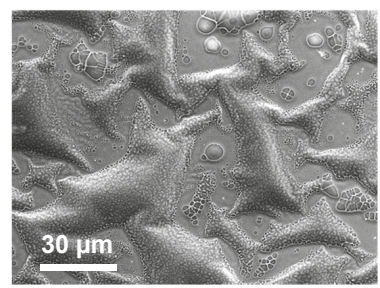

(d)

Figure 9. Top-view SEM micrographs of reference films after air annealing at $950{ }^{\circ} \mathrm{C}: \mathrm{ZrN}(\mathbf{a}) ; \mathrm{CrN}(\mathbf{b})$; $\mathrm{AlN}(\mathrm{c})$; and $\mathrm{Si}_{3} \mathrm{~N}_{4}(\mathbf{d})$. Note the different scale bar for the different images.

The $\mathrm{MeN} / \mathrm{SiN}_{\mathrm{x}}(\mathrm{Me}=\mathrm{Zr}, \mathrm{Cr}, \mathrm{Al})$ multilayered films are appreciably less subjected to surface damage after air annealing. Besides, the analysis of the surface topography of $\mathrm{CrN} / \mathrm{SiN}_{\mathrm{x}}$ and $\mathrm{AlN} / \mathrm{SiN}_{\mathrm{x}}$ multilayered films testifies to their lower susceptibility to oxidation compared to $\mathrm{ZrN} / \mathrm{SiN}_{\mathrm{x}}$ films. As an example, the SEM micrographs of $\mathrm{ZrN} / \mathrm{SiN}_{\mathrm{x}}, \mathrm{CrN} / \mathrm{SiN}_{\mathrm{x}}$ and $\mathrm{AlN} / \mathrm{SiN}_{\mathrm{x}}$ multilayered films with $5 \mathrm{~nm} / 10$ $\mathrm{nm}$ thickness ratio of elementary layers are represented in Figure 10. The lowest content of oxygen in 
$\mathrm{CrN} / \mathrm{SiN}_{\mathrm{x}}$ and $\mathrm{AlN} / \mathrm{SiN}_{\mathrm{x}}$ films after air-annealing was revealed for such thickness ratio (Figure 8). Large corroded areas were observed on the surface of $\mathrm{ZrN} / \mathrm{SiN}_{\mathrm{x}}$ coating (Figure 10a), while the surface of the $\mathrm{CrN} / \mathrm{SiN}_{\mathrm{x}}$ coating is characterized by the presence of much smaller defects, which, however, do not cause any failure of the coating integrity (Figure 10b). The surface relief of the $\mathrm{AlN} / \mathrm{SiN}_{\mathrm{x}}$ film is rather uniform (Figure 10c). Shallow, nanoscale blisters are only visible, with no sign of localized oxidation. These observations confirm the better oxidation resistance of $\mathrm{AlN} / \mathrm{SiN}_{\mathrm{x}}$ multilayers, comparatively to $\mathrm{CrN} / \mathrm{SiN}_{\mathrm{x}}$ and $\mathrm{ZrN} / \mathrm{SiN}_{\mathrm{x}}$ systems.

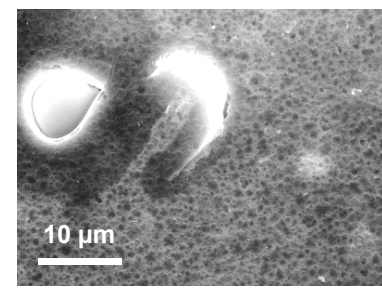

(a)

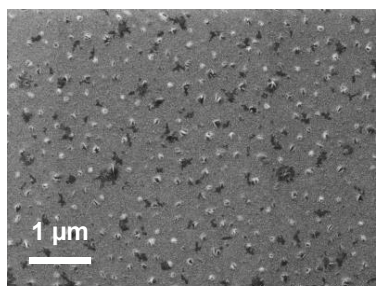

(b)

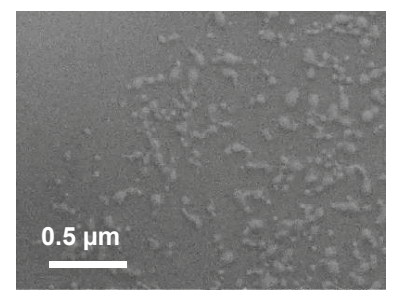

(c)

Figure 10. Top-view SEM micrographs of multilayered films with the ratio of thicknesses of MeN and $\mathrm{SiN}_{\mathrm{x}}$ elementary layers equal to $5 \mathrm{~nm} / 10 \mathrm{~nm}$ after air annealing at $950{ }^{\circ} \mathrm{C}: \mathrm{ZrN} / \mathrm{SiN}_{\mathrm{x}}(\mathbf{a}) ; \mathrm{CrN} / \mathrm{SiN}_{\mathrm{x}}(\mathbf{b})$; and $\mathrm{AlN} / \mathrm{SiN}_{\mathrm{x}}(\mathrm{c})$. Note the different scale bar for the different images.

\subsection{Discussion on the Comparative Oxidation Resistance of MeN/SiN ${ }_{x}$ Multilayers and Me-Si-N Single-Layers}

As pointed out in the Introduction, there exists two approaches for the synthesis of hard TMN coatings with enhanced phase stability during high-temperature air annealing, namely the formation of nanocomposite/amorphous Me-Si-N films or $\mathrm{MeN} / \mathrm{SiN}_{\mathrm{x}}$ multilayered films. Therefore, it is rather interesting to compare their oxidation resistance. In general, the thermal stability of $\mathrm{MeN}_{\mathrm{x}}$ phase is determined by its decomposition into Me and $\mathrm{N}_{2}(\mathrm{~g})$, which depends on the stoichiometry $\mathrm{x}=[\mathrm{N}] /[\mathrm{Me}]$. In $\mathrm{Zr}$-Si-N amorphous films, a worse thermal stability was reported for films with substoichiometric $\mathrm{ZrN}_{\mathrm{x}}<1$ phase [8]. Similar conclusions can also be made from the work of Abadias et al. on quaternary $\mathrm{TiZrAl}_{\mathrm{x}} \mathrm{N}_{\mathrm{y}}$ films [47]. However, the resistance to oxidation during air annealing of $\mathrm{MeN}_{\mathrm{x}}$ phase is also determined by its ability for crystallization of metal oxide $\left(\mathrm{MeO}_{\mathrm{x}}\right)$ phases and the type of $\mathrm{MeO}_{\mathrm{x}}$ formed (dense solid vs. volatile oxides or passivating oxides) that impact coating morphology [4,9]. The present findings show that, despite $\mathrm{CrN}$ layers being substoichiometric (see Table 1), the oxidation resistance of $\mathrm{CrN} / \mathrm{SiN}_{\mathrm{x}}$ multilayers (at least up to $950{ }^{\circ} \mathrm{C}$ ) is higher than that of $\mathrm{ZrN} / \mathrm{SiN}_{\mathrm{x}}$ multilayers (up to $860^{\circ} \mathrm{C}$ ).

Numerous works have been dedicated to study the oxidation behavior of Me-Si-N systems, including Zr-Si-N [7-10,48,49], W-Si-N [8-10], Ta-Si-N [8-10,50], Cr-Si-N [51], or Al-Si-N [8-10,52]. With increasing $\mathrm{Si}$ fraction, the microstructure typically evolves from bi-phase nanocomposites consisting of $\mathrm{MeN}_{\mathrm{x}}$ nanocrystals embedded in $\mathrm{Si}_{3} \mathrm{~N}_{4}$ amorphous phase to X-ray amorphous phase. As an example, for $\mathrm{Zr}$-Si-N coatings, the oxidation resistance rises with the increase of Si content, i.e. with increasing volume fraction of amorphous $\mathrm{Si}_{3} \mathrm{~N}_{4}$ phase $[6,48,49]$. The best oxidation resistance is achieved for amorphous coatings, typically exceeding $1000^{\circ} \mathrm{C}$ under air $[9,52]$. In the case of the $\mathrm{Zr}-\mathrm{Si}-\mathrm{N}$ system, the absence of mass gain was observed even up to the temperature of $1300{ }^{\circ} \mathrm{C}$ [9].

In a previous study [6], we compared the oxidation resistance of $\mathrm{ZrSiN}$ nanocomposite and $\mathrm{ZrN} / \mathrm{SiN}_{\mathrm{x}}$ multilayered films. It should be pointed that the investigated films were relatively thin (thickness of $\sim 300 \mathrm{~nm}$ ) that resulted in a significant fraction of the oxidized layer. When using the same deposition conditions, the oxidation starts at $700-780^{\circ} \mathrm{C}$ for $\mathrm{ZrSiN}$ nanocomposite films and at $860-950{ }^{\circ} \mathrm{C}$ for $\mathrm{ZrN} / \mathrm{SiN}_{\mathrm{x}}$ multilayered films. This testifies to the advantage of the multilayered films. The lower degree of the compressive stress relaxation at the elevated temperatures of $\mathrm{ZrSiN}$ films, as compared to $\mathrm{ZrN} / \mathrm{SiN}_{\mathrm{x}}$ multilayered films, was likely at the origin of film cracking, which 
deteriorates their oxidation resistance. The formation of cracks was indeed observed for nanocomposite and amorphous $\mathrm{ZrSiN}$ films despite reduction in oxygen penetration into the film with increasing $\mathrm{Si}$ content [6]. However, no cracks were observed for the $\mathrm{ZrN} / \mathrm{SiN}_{\mathrm{x}}$ multilayers.

\section{Summary and Conclusions}

By means of magnetron sputter deposition technique, single-layer (monolithic) $\mathrm{ZrN}, \mathrm{CrN}, \mathrm{AlN}$ and $\mathrm{Si}_{3} \mathrm{~N}_{4}$ films and $\mathrm{MeN} / \mathrm{SiN}_{\mathrm{X}}(\mathrm{Me}=\mathrm{Zr}, \mathrm{Cr}, \mathrm{Al})$ multilayered films with the different ratios of $\mathrm{MeN}$ layer thickness to $\mathrm{SiN}_{\mathrm{x}}$ layer thickness, namely $5 \mathrm{~nm} / 2 \mathrm{~nm}, 5 \mathrm{~nm} / 5 \mathrm{~nm}, 5 \mathrm{~nm} / 10 \mathrm{~nm}$ and $2 \mathrm{~nm} / 5 \mathrm{~nm}$, were synthesized. The structure and phase composition of the films in as-deposited state as well as their stability under air-annealing in the $400-950{ }^{\circ} \mathrm{C}$ temperature range were studied.

TEM, XRR and XRD results disclose the formation of periodic $\mathrm{MeN} / \mathrm{SiN}_{\mathrm{x}}$ multilayered structures characterized by uniform alternation of nanocrystalline MeN layers and amorphous $\operatorname{SiN}_{\mathrm{x}}$ layers with sharp and planar interfaces. In the case of $\mathrm{ZrN} / \mathrm{SiN}_{\mathrm{x}}$ and $\mathrm{CrN} / \mathrm{SiN}_{\mathrm{x}}$ systems, the MeN phase has (200) preferred orientation of the crystallites. In the case of $\mathrm{AlN} / \mathrm{SiN} \mathrm{N}_{\mathrm{x}}$ film, the (002) preferred orientation was observed. For all multilayered films, the decrease in crystallites size of MeN phase occurs, and this phase becomes X-ray amorphous (as well as $\mathrm{SiN}_{\mathrm{x}}$ phase) when reducing the thickness of the corresponding elementary layer down to $2 \mathrm{~nm}$.

The results of XRD, WDS and SEM analysis point that all reference monolithic films are subjected to significant oxidation level during high-temperature annealing. $\mathrm{ZrN}$ oxidizes into $\mathrm{t}-\mathrm{ZrO}_{2}$ at $550{ }^{\circ} \mathrm{C}$, while $\mathrm{CrN}$ first decomposes into $\mathrm{Cr}_{2} \mathrm{~N}$ at $550{ }^{\circ} \mathrm{C}$, followed by the formation of $\mathrm{t}-\mathrm{Cr}_{2} \mathrm{O}_{3}$ phase at 700 ${ }^{\circ} \mathrm{C}$. AlN and $\mathrm{Si}_{3} \mathrm{~N}_{4}$ films appear more thermally stable, with lower oxygen uptake at $950{ }^{\circ} \mathrm{C}$ and no crystalline oxides detected. Compared to $\mathrm{MeN}$ single-layers, $\mathrm{MeN} / \mathrm{SiN}_{\mathrm{x}}$ multilayers exhibit improved oxidation resistance due to the presence of amorphous $\mathrm{Si}_{3} \mathrm{~N}_{4}$ layers: $\mathrm{ZrN} / \mathrm{SiN}_{\mathrm{x}}$ multilayers start to oxidize at the temperatures of $780-860{ }^{\circ} \mathrm{C}$, while $\mathrm{CrN} / \mathrm{SiN}_{\mathrm{x}}$ and $\mathrm{AlN} / \mathrm{SiN}_{\mathrm{x}}$ multilayered films are stable up to $950{ }^{\circ} \mathrm{C}$. Further investigations at higher temperatures would be required to assess the upper temperature limit of their stability in air, and evaluate their performance comparatively to amorphous ternary Me-Si-N films, which are thermally stable and oxidation resistant up to $1300{ }^{\circ} \mathrm{C}$ [9].

For $\mathrm{ZrN} / \mathrm{SiN}_{\mathrm{x}}$ multilayered films, both the reduction of $f_{\mathrm{MeN}}$ fraction and increase in number of the bilayers in the film improve their oxidation resistance. However, $\mathrm{ZrN} / \mathrm{SiN}_{\mathrm{x}}$ multilayered films are least thermally stable among the three studied systems. The minimum oxygen content (27.5 at. \%) after air annealing is found for the $\mathrm{ZrN}_{\mathrm{SiN}}(2 \mathrm{~nm} / 5 \mathrm{~nm})$ film.

The $\mathrm{CrN} / \mathrm{SiN}_{\mathrm{x}}$ and $\mathrm{AlN} / \mathrm{SiN}_{\mathrm{x}}$ multilayered films are characterized by appreciably higher stability of their phase composition during air annealing. The oxygen content after annealing is in the 17.2-23.5 at.\% (CrN/SiN ${ }_{x}$ films) and 8.0-18.2 at.\% (AlN/SiN ${ }_{x}$ films) range. Both the passivating role of the chromium (or, especially, aluminum) oxides and the decrease in the compressive stresses during annealing (stress relaxation) are apparently the main reasons for the improvement of film properties. In contrast to $\mathrm{ZrN} / \mathrm{SiN}_{\mathrm{x}}$ films, for $\mathrm{CrN} / \mathrm{SiN}_{\mathrm{x}}$ and $\mathrm{AlN} / \mathrm{SiN}_{\mathrm{x}}$ multilayered films, the decrease in MeN layer thickness down to $2 \mathrm{~nm}$ leads to certain deterioration of their oxidation resistance. This can be connected to the fact that the passivating role of the chromium or aluminum oxides in the surface layers of multilayered film is less effective at such a small thickness of elementary layer.

The $\mathrm{CrN} / \mathrm{SiN}_{\mathrm{x}}$ and $\mathrm{AlN} / \mathrm{SiN}_{\mathrm{x}}$ multilayered films with the thickness ratios of elementary layers of $5 \mathrm{~nm} / 5 \mathrm{~nm}$ and $5 \mathrm{~nm} / 10 \mathrm{~nm}$ are the most promising for practical applications at elevated temperatures (up to $950^{\circ} \mathrm{C}$ ). The absence of the explicit corrosion sites on the surface is evidenced for $\mathrm{AlN} / \mathrm{SiN}_{\mathrm{x}}$ films. Among the studied samples, the lowest oxidation level was obtained for the $\mathrm{AlN}_{\mathrm{N}} \mathrm{SiN}_{\mathrm{x}}(5 \mathrm{~nm} / 10$ $\mathrm{nm}$ ) multilayer, for which the oxygen content after air annealing is only 8.0 at.\%.

Author Contributions: Conceptualization, G.A. and V.U.; methodology, V.U. and A.J.v.V.; investigation, G.A., I.S., and J.H.O.; data curation, I.S. and S.Z.; visualization, I.S.; writing-original draft preparation, I.S.; writing-review and editing, G.A.; supervision, G.A.; and funding acquisition, V.U. All authors have read and agreed to the published version of the manuscript. 
Funding: The work was partially supported by Belarusian Republican Foundation of Fundamental Research (Project F18MC-027).

Acknowledgments: The authors are grateful to Ph. Guérin for his technical assistance and scientific support on optimizing reactive growth process.

Conflicts of Interest: The authors declare no conflict of interest. The funders had no role in the design of the study; in the collection, analyses, or interpretation of data; in the writing of the manuscript, or in the decision to publish the results.

\section{References}

1. Milosev, I.; Strehblow, H.-H.; Navinsek, B. Comparison of TiN, ZrN and CrN hard nitride coatings: Electrochemical and thermal oxidation. Thin Solid Films 1997, 303, 246-254. [CrossRef]

2. Vaz, F.; Ferreira, J.; Ribeiro, E.; Rebouta, L.; Lanceros-Mendez, S.; Mendes, J.A.; Alves, E.; Goudeau, P.; Riviere, J.P.; Ribeiro, F.; et al. Influence of nitrogen content on the structural, mechanical and electrical properties of TiN thin films. Surf. Coat. Technol. 2005, 191, 317-323. [CrossRef]

3. Wen, F.; Meng, Y.D.; Ren, Z.X.; Shu, X.S. Microstructure, hardness and corrosion resistance of ZrN films prepared by inductively coupled plasma enhanced RF magnetron sputtering. Plasma Sci. Technol. 2008, 10, 170-175. [CrossRef]

4. Abadias, G.; Koutsokeras, L.E.; Siozios, A.; Patsalas, P. Stress, phase stability and oxidation resistance of ternary Ti-Me-N (Me = Zr, Ta) hard coatings. Thin Solid Films 2013, 538, 56-70. [CrossRef]

5. Barshilia, H.C.; Deepthi, B.; Arun Prabhu, A.S.; Rajam, K.S. Superhard nanocomposite coatings of TiN/Si 3 N prepared by reactive direct current unbalanced magnetron sputtering. Surf. Coat. Technol. 2006, 201, 329-337. [CrossRef]

6. Saladukhin, I.A.; Abadias, G.; Uglov, V.V.; Zlotski, S.V.; Michel, A.; Vuuren, A.J. Thermal stability and oxidation resistance of $\mathrm{ZrSiN}$ nanocomposite and $\mathrm{ZrN} / \mathrm{SiN}_{\mathrm{x}}$ multilayered coatings: A comparative study. Surf. Coat. Technol. 2017, 332, 428-439. [CrossRef]

7. Silva Neto, P.C.; Freitas, F.G.R.; Fernandez, D.A.R.; Carvalho, R.G.; Felix, L.C.; Tertoa, A.R.; Hubler, R.; Mendes, F.M.T.; Silva Junior, A.H.; Tentardini, E.K. Investigation of microstructure and properties of magnetron sputtered Zr-Si-N thin films with different Si content. Surf. Coat. Technol. 2018, 353, 355-363. [CrossRef]

8. Musil, J.; Vlček, J.; Zeman, P. Hard amorphous nanocomposite coatings with oxidation resistance above $1000{ }^{\circ}$ C. Adv. Appl. Ceram. 2008, 107, 148-154. [CrossRef]

9. Musil, J. Hard nanocomposite coatings: Thermal stability, oxidation resistance and toughness. Surf. Coat. Technol. 2012, 207, 50-65. [CrossRef]

10. Musil, J. Advanced Hard Nanocoatings: Present State and Trends. In Top 5 Contributions in Molecular Sciences, 6th ed.; Avid Science; Telanga India: Berlin, Germany, 2020; pp. 2-65.

11. Abadias, G.; Michel, A.; Tromas, C.; Jaouen, C.; Dub, S.N. Stress, interfacial effects and mechanical properties of nanoscale multilayered coatings. Surf. Coat. Technol. 2007, 202, 844-853. [CrossRef]

12. Bobzin, K.; Brögelmann, T.; Kruppe, N.C.; Arghavani, M.; Mayer, J.; Weirich, T.E. Plastic deformation behavior of nanostructured CrN/AIN multilayer coatings deposited by hybrid dcMS/HPPMS. Surf. Coat. Technol. 2017, 332, 253-261. [CrossRef]

13. Chang, Y.-Y.; Weng, S.-Y.; Chen, C.-H.; Fu, F.-X. High temperature oxidation and cutting performance of AlCrN, TiVN and multilayered AlCrN/TiVN hard coatings. Surf. Coat. Technol. 2017, 332, 494-503. [CrossRef]

14. Contreras, E.; Galindez, Y.; Rodas, M.A.; Bejarano, G.; Gómez, M.A. CrVN/TiN nanoscale multilayer coatings deposited by DC unbalanced magnetron sputtering. Surf. Coat. Technol. 2017, 332, 214-222. [CrossRef]

15. Pogrebnjak, A.; Smyrnova, K.; Bondar, O. Nanocomposite Multilayer Binary Nitride Coatings Based on Transition and Refractory Metals: Structure and Properties. Coatings 2019, 9, 155. [CrossRef]

16. Lei, Z.; Liu, Y.; Ma, F.; Song, Z.; Li, Y. Oxidation resistance of TiAlN/ZrN multilayer coatings. Vacuum 2016, 127, 22-29. [CrossRef]

17. Xiao, B.; Li, H.; Mei, H.; Dai, W.; Zuo, F.; Wu, Z.; Wang, Q. A study of oxidation behavior of AlTiN-and AlCrN-based multilayer coatings. Surf. Coat. Technol. 2018, 333, 229-237. [CrossRef]

18. Kong, M.; Zhao, W.; Wei, L.; Li, G. Investigations on the microstructure and hardening mechanism of TiN/Si ${ }_{3} \mathrm{~N}_{4}$ nanocomposite coatings. J. Phys. D Appl. Phys. 2007, 40, 2858. [CrossRef] 
19. Wu, Z.; Zhong, X.; Liu, C.; Wang, Z.; Dai, W.; Wang, Q. Plastic Deformation Induced by Nanoindentation Test Applied on $\mathrm{ZrN} / \mathrm{Si}_{3} \mathrm{~N}_{4}$ Multilayer Coatings. Coatings 2018, 8, 11. [CrossRef]

20. Bai, X.; Zheng, W.; An, T.; Jiang, Q. Effects of deposition parameters on microstructure of $C r N / S_{3} \mathrm{~N}_{4}$ nanolayered coatings and their thermal stability. J. Phys. Condens. Matter 2005, 17, 6405-6413. [CrossRef]

21. Soares, T.P.; Aguzzoli, C.; Soares, G.V.; Figueroa, C.A.; Baumvol, I.J.R. Physicochemical and mechanical properties of crystalline/amorphous $\mathrm{CrN} / \mathrm{Si}_{3} \mathrm{~N}_{4}$ multilayers. Surf. Coat. Technol. 2013, 237, 170-175. [CrossRef]

22. Hultman, L.; Bareño, J.; Flink, A.; Söderberg, H.; Larsson, K.; Petrova, V.; Odén, M.; Greene, J.E.; Petrov, I. Interface structure in superhard TiN-SiN nanolaminates and nanocomposites: Film growth experiments and ab initio calculations. Phys. Rev. B 2007, 75, 155437. [CrossRef]

23. Abadias, G.; Uglov, V.V.; Saladukhin, I.A.; Zlotski, S.V.; Tolmachova, G.; Dub, S.N.; Vuuren, A.J. Growth, structural and mechanical properties of magnetron-sputtered $\mathrm{ZrN} / \mathrm{SiN}_{\mathrm{x}}$ nanolaminated coatings. Surf. Coat. Technol. 2016, 308, 158-167. [CrossRef]

24. Söderberg, H.; Odén, M.; Larsson, T.; Hultman, L.; Molina-Adareguia, J.M. Epitaxial stabilization of cubic-SiN ${ }_{x}$ in TiN/SiN ${ }_{x}$ multilayers. Appl. Phys. Lett. 2006, 88, 191902. [CrossRef]

25. Dong, Y.; Zhao, W.; Yue, J.; Li, G. Crystallization of $\mathrm{Si}_{3} \mathrm{~N}_{4}$ layers and its influences on the microstructure and mechanical properties of $\mathrm{ZrN} / \mathrm{Si}_{3} \mathrm{~N}_{4}$ nanomultilayers. Appl. Phys. Lett. 2006, 89, 121916. [CrossRef]

26. Ghafoor, N.; Lind, H.; Tasnardi, F.; Abrikosov, I.A.; Odern, M. Anomalous epitaxial stability of (001) interfaces in $\mathrm{ZrN} / \mathrm{SiN}_{\mathrm{x}}$ multilayers. APL Mater. 2014, 2, 046106. [CrossRef]

27. Parlinska-Wojtan, M.; Pélisson-Schecker, A.; Hug, H.J.; Rutkowski, B.; Patscheider, J. AlN/Si ${ }_{3} \mathrm{~N}_{4}$ multilayers as an interface model system for $\mathrm{Al}_{1-x} \mathrm{Si}_{x} \mathrm{~N} / \mathrm{Si}_{3} \mathrm{~N}_{4}$ nanocomposite thin films. Surf. Coat. Technol. 2015, 261, 418-425. [CrossRef]

28. Huang, L.; Chen, Z.Q.; Liu, W.B.; Huang, P.; Meng, X.K.; Xu, K.W.; Wang, F.; Lu, T.J. Enhanced irradiation resistance of amorphous alloys by introducing amorphous/amorphous interfaces. Intermetallics 2019, 107, 39-46. [CrossRef]

29. Mège-Revil, A.; Steyer, P.; Cardinal, S.; Thollet, G.; Esnouf, C.; Jacquot, P.; Stauder, B. Correlation between thermal fatigue and thermomechanical properties during the oxidation of multilayered TiSiN nanocomposite coatings synthesized by a hybrid physical/chemical vapour deposition process. Thin Solid Films 2010, 518, 5932-5937. [CrossRef]

30. Colin, J.J.; Diot, Y.; Guerin, P.; Lamongie, B.; Berneau, F.; Michel, A.; Jaouen, C.; Abadias, G. A load-lock compatible system for in situ electrical resistivity measurements during thin film growth. Rev. Sci. Instrum. 2016, 87, 023902. [CrossRef]

31. Abadias, G.; Koutsokeras, L.E.; Dub, S.N.; Tolmachova, G.N.; Debelle, A.; Sauvage, T.; Villechaise, P. Reactive magnetron cosputtering of hard and conductive ternary nitride thin films: Ti-Zr-N and Ti-Ta-N. J. Vac. Sci. Technol. A 2010, 28, 541-551. [CrossRef]

32. Simonot, L.; Babonneau, D.; Camelio, S.; Lantiat, D.; Guérin, P.; Lamongie, B.; Antad, V. In situ optical spectroscopy during deposition of $\mathrm{Ag}: \mathrm{Si}_{3} \mathrm{~N}_{4}$ nanocomposite films by magnetron sputtering. Thin Solid Films 2010, 518, 2637-2643. [CrossRef]

33. Abadias, G.; Chason, E.; Keckes, J.; Sebastiani, M.; Thompson, G.B.; Barthel, E.; Doll, G.L.; Murray, C.E.; Stoessel, C.H.; Martinu, L. Review Article: Stress in thin films and coatings: Current status, hallenges, and prospects. J. Vac. Sci. Technol. A 2018, 36, 20801. [CrossRef]

34. Parratt, L.G. Surface Studies of Solids by Total Reflection of X-Rays. Phys. Rev. 1954, 95, 359-369. [CrossRef]

35. Koutsokeras, L.E.; Abadias, G. Intrinsic stress in $\mathrm{ZrN}$ thin films: Evaluation of grain boundary contribution from in situ wafer curvature and ex situ X-ray diffraction techniques. J. Appl. Phys. 2012, 111, 093509. [CrossRef]

36. Abadias, G.; Ivashchenko, V.I.; Belliard, L.; Djemia, P. Structure, phase stability and elastic properties in the $\mathrm{Ti}_{1-\mathrm{x}} \mathrm{Zr}_{\mathrm{x}} \mathrm{N}$ thin-film system: Experimental and computational studies. Acta Mater. 2012, 60, 5601-5614. [CrossRef]

37. Patsalas, P.; Kalfagiannis, N.; Kassavetis, S.; Abadias, G.; Bellas, R.V.; Lekka, C.; Lidorikis, E. Conductive nitrides: Growth principles, optical and electronic properties, and their perspectives in photonics and plasmonics. Mater. Sci. Eng. R 2018, 123, 1-55. [CrossRef]

38. Musil, J. Flexible Hard Nanocomposite Coatings. RSC Adv. 2015, 5, 60482-60495. [CrossRef] 
39. Xingrun, R.; Zhu, H.; Meixia, L.; Jiangao, Y.; Hao, C. Comparison of microstructure and tribological behaviors of CrAlN and CrN film deposited by DC magnetron sputtering. Rare Met. Mater. Eng. 2018, 47, 1100-1106. [CrossRef]

40. Ren, X.; Zhang, Q.; Huang, X.; Su, W.; Yang, J.; Chen, H. Microstructure and tribological properties of CrN films deposited by direct current magnetron sputtering. Rare Met. Mater. Eng. 2018, 47, 2283-2289. [CrossRef]

41. Khan, S.; Shahid, M.; Mahmood, A.; Shah, A.; Ahmed, I.; Mehmood, M.; Aziz, U.; Raza, Q.; Alam, M. Texture of the nano-crystalline AIN thin films and the growth conditions in DC magnetron sputtering. Prog. Nat. Sci. Mater. Int. 2015, 25, 282-290. [CrossRef]

42. Taurino, A.; Signore, M.A.; Catalano, M.; Kim, M.J. (101) and (002) oriented AlN thin films deposited by sputtering. Mater. Lett. 2017, 200, 18-20. [CrossRef]

43. Signore, M.A.; Taurino, A.; Valerini, D.; Rizzo, A.; Farella, I.; Catalano, M.; Quaranta, F.; Siciliano, P. Role of oxygen contaminant on the physical properties of sputtered AlN thin films. J. Alloys Compd. 2015, 649, 1267-1272. [CrossRef]

44. Riah, B.; Ayad, A.; Camus, J.; Rammal, M.; Boukari, F.; Chekour, L.; Djouadi, M.A.; Rouag, N. Textured hexagonal and cubic phases of AlN films deposited on Si (100) by DC magnetron sputtering and high power impulse magnetron sputtering. Thin Solid Films 2018, 655, 34-40. [CrossRef]

45. Mayrhofer, P.H.; Rovere, F.; Moser, M.; Strondl, C.; Tietema, R. Thermally induced transitions of CrN thin films. Scr. Mater. 2007, 57, 249-252. [CrossRef]

46. Lin, J.; Moore, J.J.; Wang, J.; Sproul, W.D. High temperature oxidation behavior of CrN/AlN superlattice films. Thin Solid Films 2011, 519, 2402-2408. [CrossRef]

47. Abadias, G.; Saladukhin, I.A.; Uglov, V.V.; Zlotski, S.V.; Eyidi, D. Thermal stability and oxidation behavior of quaternary TiZrAlN magnetron sputtered thin films: Influence of the pristine microstructure. Surf. Coat. Technol. 2013, 237, 187-195. [CrossRef]

48. Pilloud, D.; Pierson, J.F.; Marco de Lucas, M.C.; Alnot, M. Stabilisation of tetragonal zirconia in oxidized Zr-Si-N nanocomposite coatings. Appl. Surf. Sci. 2004, 229, 132-139. [CrossRef]

49. Chen, Y.-I.; Chang, S.-C.; Chang, L.-C. Oxidation resistance and mechanical properties of Zr-Si-N coatings with cyclic gradient concentration. Surf. Coat. Technol. 2017, 320, 168-173. [CrossRef]

50. Chen, Y.-I.; Gao, Y.-X.; Chang, L.-C. Oxidation behavior of Ta-Si-N coatings. Surf. Coat. Technol. 2017, 332, 72-79. [CrossRef]

51. Mikula, M.; Grančič, B.; Drienovský, M.; Satrapinskyy, L.; Roch, T.; Hájovská, Z.; Gregor, M.; Plecenik, T.; Čička, R.; Plecenik, A.; et al. Thermal stability and high-temperature oxidation behavior of Si-Cr-N coatings with high content of silicon. Surf. Coat. Technol. 2013, 232, 349-356. [CrossRef]

52. Musil, J.; Remnev, G.; Legostaev, V.; Uglov, V.; Lebedynskiy, A.; Lauk, A.; Procházka, J.; Haviar, S.; Smolyanskiy, E. Flexible hard Al-Si-N films for high temperature operation. Surf. Coat. Technol. 2016, 307, 1112-1118. [CrossRef]

(C) 2020 by the authors. Licensee MDPI, Basel, Switzerland. This article is an open access article distributed under the terms and conditions of the Creative Commons Attribution (CC BY) license (http://creativecommons.org/licenses/by/4.0/). 

MDPI

St. Alban-Anlage 66

4052 Basel

Switzerland

Tel. +41616837734

Fax +41 613028918

www.mdpi.com

Coatings Editorial Office

E-mail: coatings@mdpi.com www.mdpi.com/journal/coatings

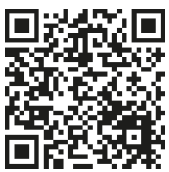



MDPI

St. Alban-Anlage 66

4052 Basel

Switzerland

Tel: +41 616837734

Fax: +41 613028918

www.mdpi.com 